REVIEWS OF MODERN PHYSICS, VOLUME 91， JANUARY-MARCH 2019

\title{
Magnetic small-angle neutron scattering
}

\author{
Sebastian Mühlbauer ${ }^{*}$ \\ Heinz Maier-Leibnitz Zentrum (MLZ), Technische Universität München, \\ $D$-85748 Garching, Germany
}

Dirk Honecker

Institut Laue-Langevin, 71 avenue des Martyrs, F-38042 Grenoble, France

Élio A. Périgo

ABB Corporate Research Center, 940 Main Campus Drive, Raleigh, North Carolina 27606, USA

\section{Frank Bergner}

Institute of Resource Ecology, Helmholtz-Zentrum Dresden-Rossendorf, D-01328 Dresden, Germany

\section{Sabrina Disch}

Universität zu Köln, Department für Chemie, Luxemburger Straße 116, D-50939 Köln, Germany

\section{André Heinemann}

German Engineering Materials Science Centre (GEMS)

at Heinz Maier-Leibnitz Zentrum (MLZ), Helmholtz-Zentrum Geesthacht GmbH, D-85748 Garching, Germany

\author{
Sergey Erokhin and Dmitry Berkov \\ General Numerics Research Lab, Moritz-von-Rohr-Straße 1A, D-07745, Jena, Germany
}

Chris Leighton

Department of Chemical Engineering and Materials Science, University of Minnesota, Minneapolis, Minnesota 55455, USA

\section{Morten Ring Eskildsen}

Department of Physics, University of Notre Dame, Notre Dame, Indiana 46556, USA

\author{
Andreas Michels ${ }^{\dagger}$ \\ Physics and Materials Science Research Unit, University of Luxembourg, \\ 162A Avenue de la Faïencerie, L-1511 Luxembourg, Grand Duchy of Luxembourg \\ (published 4 March 2019)
}

Small-angle neutron scattering (SANS) is one of the most important techniques for microstructure determination, being utilized in a wide range of scientific disciplines, such as materials science, physics, chemistry, and biology. The reason for its great significance is that conventional SANS is probably the only method capable of probing structural inhomogeneities in the bulk of materials on a mesoscopic real-space length scale from roughly 1 to $300 \mathrm{~nm}$. Moreover, the exploitation of the spin degree of freedom of the neutron provides SANS with a unique sensitivity to study magnetism and magnetic materials at the nanoscale. As such, magnetic SANS ideally complements more real-space and surface-sensitive magnetic imaging techniques, e.g., Lorentz transmission electron microscopy, electron holography, magnetic force microscopy, Kerr microscopy, or spin-polarized scanning

\footnotetext{
*Sebastian.Muehlbauer@frm2.tum.de

${ }^{\dagger}$ Andreas.Michels@uni.lu
} 
tunneling microscopy. This review summarizes the recent applications of the SANS method to study magnetism and magnetic materials. This includes a wide range of materials classes from nanomagnetic systems such as soft magnetic Fe-based nanocomposites, hard magnetic Nd-Fe-B-based permanent magnets, magnetic steels, ferrofluids, nanoparticles, and magnetic oxides to more fundamental open issues in contemporary condensed matter physics such as skyrmion crystals, noncollinear magnetic structures in noncentrosymmetric compounds, magnetic or electronic phase separation, and vortex lattices in type-II superconductors. Special attention is paid not only to the vast variety of magnetic materials and problems where SANS has provided direct insight, but also to the enormous progress made regarding the micromagnetic simulation of magnetic neutron scattering.

DOI: $10.1103 /$ RevModPhys.91.015004

\section{CONTENTS}

I. Introduction

II. Magnetic SANS: Basics

A. Description of the SANS setup

B. SANS cross sections

1. General considerations

2. Unpolarized SANS

3. Half-polarized SANS (SANSPOL)

4. Polarized SANS (POLARIS)

C. Magnetic SANS theory

D. Relation to conventional particle-matrix approach

III. Nd-Fe-B-based Permanent Magnets

A. Correlation function of the spin-misalignment SANS cross section

B. Selected results on Nd-Fe-B magnets

IV. Magnetic Steels

A. New steels for application under extreme conditions

B. Prerequisites

C. $\mathrm{Fe}-\mathrm{Cu}$ alloys and reactor pressure vessel steels

D. Fe-Cr alloys and ferritic-martensitic $\mathrm{Cr}$ steels

E. Advanced oxide dispersion-strengthened steels

F. Summary on magnetic steels

V. Magnetic Nanoparticles and Ferrofluids

A. Particle-matrix approach

B. Intraparticle magnetization

C. Interparticle correlations

D. Anisotropic nanostructures

VI. Micromagnetic Simulations

A. Novel micromagnetic simulation methodology for modeling bulk magnetic materials

B. Simulation of magnetic neutron scattering: Decrypting SANS cross sections

VII. SANS as a Probe of Nanoscale Magnetic Inhomogeneity in Complex Magnetic Systems

A. Magnetic inhomogeneity and magnetic phase separation

B. Complex magnetic oxides

1. Perovskite manganites

2. Perovskite cobaltites

C. Complex magnetic alloys

VIII. Skyrmion Lattices and Noncollinear Spin Structures

A. Noncollinear magnetic structures

1. Frustrated and oscillating interactions

2. Dzyaloshinskii-Moriya interaction

3. Multiferroic properties of spiral magnets

4. Noncollinear magnetism and diffraction in SANS geometry

5. Properties of B20 spiral magnets as inferred from SANS
6. Properties of non-B20 spiral magnets as inferred from SANS

B. Magnetic skyrmions

1. The concept of skyrmions

2. Skyrmions in spiral magnets seen by SANS

IX. Vortex Lattices in Superconductors

A. Imaging the vortex lattice by neutron diffraction

B. Vortices as probes of superconducting materials

1. VL symmetry and orientation

2. Field and temperature dependence of the VL form factor

3. Complex order parameters, multigap superconductivity, and Pauli limiting 52

C. Vortex matter studies 53

1. Structural properties and correlations 53

2. Relating structural and dynamic properties $\quad 54$

3. Ordered, nonequilibrium VL phases 54

X. Concluding Remarks 55

Acknowledgments $\quad 57$

References $\quad 57$

\section{INTRODUCTION}

Small-angle neutron scattering (SANS) is a particularly powerful and unique technique, which allows one to investigate microstructural (density and composition) as well as magnetic inhomogeneities in the volume of materials and on a mesoscopic length scale between a few and a few hundred nanometers. This is a size regime in which many macroscopic material properties are realized. From a historical point of view, experimental and theoretical progress in the domain of small-angle scattering is closely connected to the development of laboratory small-angle x-ray scattering (SAXS) methods (Guinier and Fournet, 1955). Since SANS and its X-ray counterpart are well developed and widely acknowledged in diverse fields of science, such as materials science, physics, chemistry, and biology, there exists an enormous body of research literature. The standard references for nuclear (nonmagnetic) SANS and SAXS are the well-known textbooks by Guinier and Fournet (1955), Glatter and Kratky (1982), Feigin and Svergun (1987), Svergun et al. (2013), and Gille (2014). For reviews on various topics of small-angle scattering, for instance, on polymers, disordered and porous materials, colloidal systems, ferrofluids, magnetic materials, superconductors, ceramics, biological structures, and precipitates in metallic alloys and composites, see Schmatz et al. (1974), Jacrot (1976), Gerold and Kostorz (1978), Higgins and Stein (1978), Schelten and Hendricks (1978), Chen and Lin (1987), 
Martin and Hurd (1987), Bates (1988), Chen et al. (1988), Hayter (1988), Page (1988), Kostorz (1991, 2014), Schmidt (1991), Pedersen (1997), Wiedenmann (2002, 2010), Fratzl (2003), Svergun and Koch (2003), Thiyagarajan (2003), Fitzsimmons et al. (2004), Radlinski et al. (2004), Stuhrmann (2004), Allen (2005), Wagner and Kohlbrecher (2005), Wignall and Melnichenko (2005), Fritz and Glatter (2006), Melnichenko and Wignall (2007), Michels and Weissmüller (2008), Avdeev and Aksenov (2010), Hammouda (2010), Eskildsen, Forgan, and KawanoFurukawa (2011), Laver (2012), Hollamby (2013), Pauw (2013), Michels (2014), Michels et al. (2014), and Avdeev et al. (2015).

Only with the advent of high-brilliance neutron sources and the concomitant development of the first dedicated SANS instruments (Schmatz et al., 1974; Ibel, 1976) did it become possible to explore magnetism and superconductivity on a mesoscopic length scale by means of SANS. With magnetic SANS playing a pivotal role, the rapidly evolving progress in the field of magnetism and superconductivity (Brandt, 1995; Skomski, 2003; Bader, 2006; Sellmyer and Skomski, 2006; Bauer and Pfleiderer, 2010; Furrer and Waldmann, 2013; Nagaosa and Tokura, 2013; Nisoli, Moessner, and Schiffer, 2013; Hellman et al., 2017) is naturally accompanied by the quest to resolve ever finer details of the magnetic microstructure. As such, SANS ideally complements well-known and established methods for characterizing and analyzing the static and dynamic spin structure of nanomaterials, including neutron diffraction and spectroscopy (Chatterji, 2006; Furrer, Mesot, and Strässle, 2009), Lorentz and Kerr microscopy (Hubert and Schäfer, 1998), magnetic force microscopy (Koblischka and Hartmann, 2003; Meyer, Hug, and Bennewitz, 2004), spin-polarized scanning tunneling microscopy (Wiesendanger, 2009, 2016), or x-ray magnetic circular dichroism in combination with photoelectron emission microscopy (Locatelli and Bauer, 2008; Cheng and Keavney, 2012; Bauer, 2014).

In this review we provide a summary of the recent applications of the SANS method to study magnetism and magnetic materials. This covers many of the most important classes of magnetic materials and addresses a wide range of topics from fundamental questions in condensed matter physics to applied materials science. The review is organized as follows: Sec. II revisits the basics of "classical" diffuse magnetic SANS, including a summary of recent theoretical and experimental progress regarding the spin structures of polycrystalline bulk ferromagnets and its relation to the conventional particle-matrix approach. We provide a compilation of the various unpolarized, half-polarized, and polarized SANS cross sections. Sections III and IV highlight the recent discoveries concerning the investigation of $\mathrm{Nd}-\mathrm{Fe}-\mathrm{B}$-based permanent magnets and magnetic steels, whereas Sec. V covers magnetic nanoparticles and ferrofluids. In Sec. VI, we review the recent progress made in using full-scale micromagnetic simulations for the understanding of the fundamentals of magnetic SANS on multiphase systems. Section VII is concerned with the application of the magnetic SANS method to study complex magnetic systems which exhibit nanoscale magnetic inhomogeneity. These include magnetically and electronically phase-separated complex oxides and metal alloys, which have recently been extensively studied with SANS. Sections VIII and IX summarize the state of the art of SANS research on skyrmion lattices, long-range noncollinear magnetic structures, and vortex lattices (VLs) in type-II superconductors. Finally, Sec. X provides a brief summary and gives an outlook on future developments and challenges. In order to keep the sections self-contained, each section provides an introductory paragraph for the reader.

\section{MAGNETIC SANS: BASICS}

We begin this section with a description of a typical SANS setup in Sec. II.A. In Sec. II.B the basic expressions for the various unpolarized and spin-polarized elastic SANS cross sections $d \Sigma / d \Omega$ will be displayed. We focus on the two most relevant scattering geometries which have the applied magnetic field $\mathbf{H}_{0}$ either perpendicular or parallel to the wave vector $\mathbf{k}_{0}$ of the incoming neutron beam. In the first Born approximation (Messiah, 1990), the magnetic contribution to $d \Sigma / d \Omega$ is fully determined by the three Cartesian Fourier components $\widetilde{M}_{x, y, z}(\mathbf{q})$ of the magnetization vector field $M_{x, y, z}(\mathbf{r})$ of the sample. Using the continuum theory of micromagnetics (Sec. II.C), the functions $\widetilde{M}_{x, y, z}(\mathbf{q})$ can be computed for bulk ferromagnets in the small-misalignment approximation, in this way providing closed-form expressions for any desired $d \Sigma / d \Omega$ as a function of momentum-transfer vector $\mathbf{q}$, applied magnetic field, magnetic-interaction parameters (exchange, anisotropy, magnetostatics), and microstructural quantities such as particle size, shape, and texture; selected experimental data will be discussed in order to underline the theoretical approach. Finally, Sec. II.D establishes the connection to the conventional particle-matrix description of magnetic SANS, which assumes homogeneously magnetized domains. It is emphasized that the SANS cross sections which are introduced in this section are the ones for diffuse magnetic SANS, while the well-known equations for elastic magnetic Bragg diffraction, relevant for the discussion of spiral magnetic structures, skyrmions, or vortex lattices in superconductors, are introduced in Sec. VIII.

\section{A. Description of the SANS setup}

Figure 1 depicts the typical SANS setup along with schematics of the two most commonly used scattering geometries. By means of a mechanical velocity selector or chopperbased time-of-flight methods the incoming wavelength band (typically $\lambda \sim 3-30 \AA$ ) is selected from a cold neutron beam (energy range $\sim 0.1-10 \mathrm{meV} \sim 1-120 \mathrm{~K}$ ) (Schober, 2014), provided by a spallation or a reactor source. The mean wavelength and wavelength resolution can be tuned $[\Delta \lambda / \lambda \sim$ $1 \%-30 \%$ (FWHM)], depending on the rotational speed and tilting angle of the selector or the duty cycle and frame overlap of the chopper system. In the evacuated presample flight path a set of apertures collimates the beam. A particular strength of the SANS technique is that experiments can be conducted under rather flexible sample environments (e.g., temperature, electric and magnetic field, pressure, neutron polarization, or timeresolved data acquisition). The typical size of the irradiated area of sample is of the order of $1 \mathrm{~cm}^{2}$. 

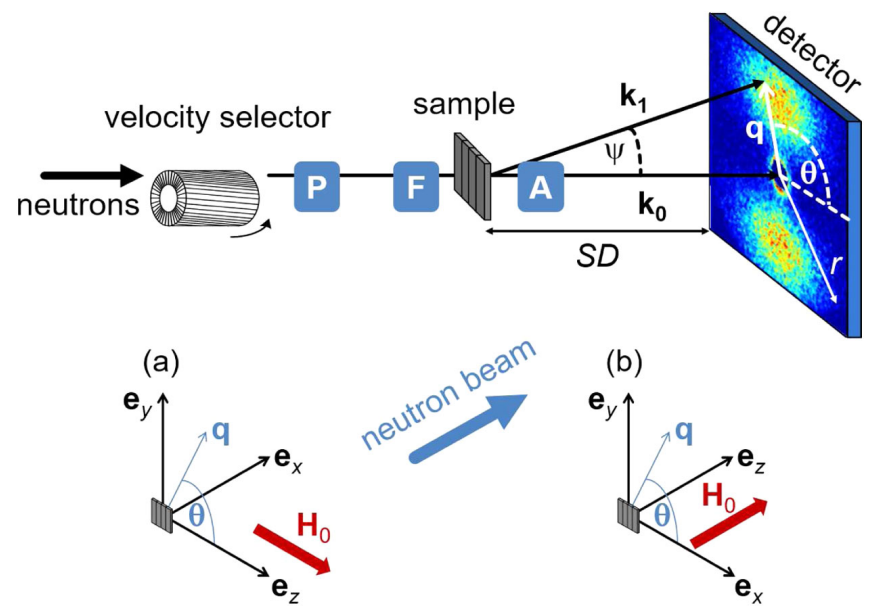

FIG. 1. Schematic of the SANS setup and the two commonly employed scattering geometries in magnetic SANS experiments. (a) $\mathbf{k}_{0} \perp \mathbf{H}_{0}$; (b) $\mathbf{k}_{0} \| \mathbf{H}_{0}$. The scattering vector $\mathbf{q}$ is defined as $\mathbf{q}=\mathbf{k}_{1}-\mathbf{k}_{0}$, where $\mathbf{k}_{0}$ and $\mathbf{k}_{1}$ are the wave vectors of the incident and scattered neutrons; $q=|\mathbf{q}|=(4 \pi / \lambda) \sin (\psi / 2)$ depends on the mean wavelength $\lambda$ of the neutrons and on the scattering angle $\psi$. The symbols "P," "F," and "A" denote, respectively, the polarizer, spin flipper, and analyzer, which are optional neutron optical devices. $S D=$ sample-to-detector distance; $\quad r=$ radial distance on the detector (measured from the beam center). SANS usually assumes elastic scattering $\left(k_{0}=k_{1}=2 \pi / \lambda\right)$, and the component of $\mathbf{q}$ along the incident neutron beam [i.e., $q_{x}$ in (a) and $q_{z}$ in (b)] is neglected. The azimuthal angle $\theta$ describes the angular anisotropy of the recorded scattering pattern on a twodimensional position-sensitive detector. The applied magnetic field $\mathbf{H}_{0}$ is always taken parallel to $\mathbf{e}_{z}$, in this way defining the longitudinal magnetization.

Two-dimensional position-sensitive detector arrays, moving along rails in an evacuated post-sample flight path (sample-to-detector distance $\sim 1-40 \mathrm{~m}$ ), count the scattered neutrons during acquisition times ranging between a few minutes and a few hours. The recorded neutron counts (in each pixel element) are corrected for detector dead time, dark current and efficiency, sample transmission, and background scattering and are normalized to incident-beam flux. A solidangle correction is applied to the data which corrects for the planar geometry of the detector (Glinka et al., 1998; Karge, Gilles, and Busch, 2017). The size of an individual pixel element of the detector is typically $\lesssim 10 \times 10 \mathrm{~mm}^{2}$, so that the related resolution effects become negligible. The scattering cross section of the sample is obtained by comparing the corrected signal to a reference sample (e.g., water, polystyrene, porous silica, or vanadium single crystal) of known cross section. The data-reduction procedure provides the macroscopic differential scattering cross section $d \Sigma / d \Omega$ of the sample in absolute units (typically $\mathrm{cm}^{-1}$ ) and as a function of the magnitude and orientation of the momentum transfer or scattering vector $\mathbf{q}$ (see Fig. 1). In order to conveniently present the neutron data, one often carries out a so-called azimuthal averaging procedure, whereby the data at a constant magnitude of $\mathbf{q}$ are integrated within a certain angular range (e.g., over $0 \leq \theta \leq 2 \pi$ ); this yields $d \Sigma / d \Omega$ as a function of $|\mathbf{q}|=q$. The uncertainty in the cross sections determined by this procedure is estimated to be 5\%-10\% (Glinka et al., 1998; Rennie et al., 2013).

When $\psi$ denotes the scattering angle [incident beam $\mathbf{k}_{0} \| \mathbf{e}_{x}$, see Fig. 1(a)], and the azimuthal angle $\theta$ is used to specify the orientation of $\mathbf{q}$ on the two-dimensional detector [with $\left.q=2 k_{0} \sin (\psi / 2)\right]$, the scattering vector is found to be

$$
\mathbf{q}=\left\{\begin{array}{l}
q_{x} \\
q_{y} \\
q_{z}
\end{array}\right\}=q\left\{\begin{array}{c}
-\sin (\psi / 2) \\
\cos (\psi / 2) \sin \theta \\
\cos (\psi / 2) \cos \theta
\end{array}\right\}=k_{0}\left\{\begin{array}{c}
\cos \psi-1 \\
\sin \psi \sin \theta \\
\sin \psi \cos \theta
\end{array}\right\} .
$$

For small-angle scattering $\psi \lesssim 5^{\circ}-10^{\circ}$, so that the magnitude of the component of $\mathbf{q}$ along the incident-beam direction $q \sin (\psi / 2)$ is much smaller than the other two components. The three-dimensional scattering vector is therefore approximated by a two-dimensional one. This approximation, which violates the condition for elastic scattering $\left(k_{0}=k_{1}\right)$, is valid for not too large scattering angles, e.g., $\left|q_{x}\right| / q=\sin (\psi / 2) \cong$ $4.4 \%$ for $\psi=5^{\circ}$ and the related error in the intensity is less than $1 \%$ (Fritz-Popovski, 2015).

Since

$$
q=\frac{4 \pi}{\lambda} \sin (\psi / 2) \cong \frac{4 \pi}{\lambda} \sin \left(\frac{1}{2} \arctan \left[\frac{r}{S D}\right]\right),
$$

where $r \sim 0.04-0.7 \mathrm{~m}$ is the radial distance on the detector (measured from the beam center), and $S D \sim 1-40 \mathrm{~m}$ denotes the sample-to-detector distance, we see that different momentum transfers can be accessed by varying $S D$ or the wavelength $\lambda \sim 3-30 \AA$. With conventional SANS instruments it becomes thus possible to cover a $q$ range of $0.01 \lesssim q \lesssim 5 \mathrm{~nm}^{-1}$, which translates into structure sizes of the order of 1-300 $\mathrm{nm}$. The $q$ resolution of a SANS instrument is mainly related to the wavelength spread of the incident neutrons, the finite collimation of the beam, and the detector resolution. Taking into account the former two contributions, it is readily verified [using $q=(4 \pi / \lambda) \sin (\psi / 2)$ ] that the rootmean-square (rms) uncertainty in $q$ is given by

$$
\begin{aligned}
\operatorname{rms}(q) & =\sqrt{\left\langle(\Delta q)^{2}\right\rangle} \\
& =\sqrt{q^{2}\left(\frac{\Delta \lambda}{\lambda}\right)^{2}+\left(k_{0}^{2}-\frac{1}{4} q^{2}\right)(\Delta \psi)^{2}} .
\end{aligned}
$$

The angular divergence $\Delta \psi$ of the beam can be determined from the measured profile of the direct beam $\left[\operatorname{rms}(q=0)=k_{0} \Delta \psi\right]$; typical values of $\Delta \psi$ are of the order of $10^{-2}$ to $10^{-3} \mathrm{rad}$. Equation (2) demonstrates that wavelength smearing dominates at large $q$, while angular-resolution effects show up at small $q$. For studies that describe the optimal instrument configuration, instrumental resolution (smearing) effects, the impact of gravitation, the data-reduction procedure, the performance of SANS instruments, or the treatment of multiple scattering, see Schelten and Schmatz (1980), Chen and Lin (1987), Pedersen, Posselt, and Mortensen (1990), Allen and Berk (1994), May (1994), 
Barker and Pedersen (1995), Glinka et al. (1998), Kohlbrecher and Wagner (2000), Mazumder et al. (2001), Saroun (2007), Dewhurst (2008), Mildner and Cubitt (2012), Dewhurst et al. (2016), and Mühlbauer, Heinemann et al. (2016).

The neutrons incident on the sample may be polarized by means of a (supermirror transmission) polarizer and the initial neutron polarization can be reverted by $180^{\circ}$ using a (radiofrequency) spin flipper (Bazhenov et al., 1993; Keller et al., 2000) (see Fig. 1). In order to discriminate the neutron-spin state after interaction with the sample, a ${ }^{3} \mathrm{He}$ spin filter (Batz et al., 2005) acts as a neutron-spin analyzer and, correspondingly, is installed behind the sample (sometimes inside the detector housing). Magnetic guide fields of the order of $1 \mathrm{mT}$ serve to maintain the polarization on the path between the polarizer and the ${ }^{3} \mathrm{He}$ filter. Progress in the development of ${ }^{3} \mathrm{He}$ spin filters (Petoukhov et al., 2006) allows one to perform routinely uniaxial (also called longitudinal or one-dimensional) neutron-polarization analysis (POLARIS) on a SANS instrument, for instance, at SANS-1 and KWS-1 at the Heinz Maier-Leibnitz Zentrum, at D22 and D33 at the Institut LaueLangevin, or at NG3 and NG7 at the NIST Center for Neutron Research. We emphasize that the above described setup of supermirror transmission polarizer $(\mathrm{P})$, rf spin flipper $(\mathrm{F})$, and ${ }^{3} \mathrm{He}$ spin analyzer (A) represents the most commonly installed configuration for uniaxial polarization analysis at SANS instruments. There exist, of course, many other neutron instrumentation devices for polarizing neutron beams and for turning the neutron-spin direction (Williams, 1988).

In a uniaxial polarization analysis (Moon, Riste, and Koehler, 1969), it becomes possible to measure four intensities that connect two neutron-spin states. The externally applied magnetic field at the sample position defines the quantization axis for both incident and scattered polarization, whereby the scattered neutron may undergo a spin-reversing event due to the magnetic interaction with the sample. Following Moon, Riste, and Koehler (1969), the four spinresolved scattering cross sections are the two non-spin-flip quantities $d \Sigma^{++} / d \Omega$ and $d \Sigma^{--} / d \Omega$ and the two spin-flip cross sections $d \Sigma^{+-} / d \Omega$ and $d \Sigma^{-+} / d \Omega$. When the rf flipper is off (inactive), we measure, depending on the spin state of the ${ }^{3} \mathrm{He}$ filter, the non-spin-flip or the spin-flip cross section $d \Sigma^{++} / d \Omega$ or $d \Sigma^{+-} / d \Omega$. Likewise, when the flipper is on, we measure either $d \Sigma^{--} / d \Omega$ or $d \Sigma^{-+} / d \Omega$. The corresponding expressions for the cross sections are denoted as the POLARIS equations (see Sec. II.B.4).

SANS experiments with a polarized incident beam only and no detection of the polarization of the scattered neutrons provide access to the half-polarized cross sections (denoted SANSPOL) $d \Sigma^{+} / d \Omega$ and $d \Sigma^{-} / d \Omega$, which combine non-spinflip and spin-flip scattering contributions. In particular (see Sec. II.B.3),

$$
\begin{aligned}
& \frac{d \Sigma^{+}}{d \Omega}=\frac{d \Sigma^{++}}{d \Omega}+\frac{d \Sigma^{+-}}{d \Omega}, \\
& \frac{d \Sigma^{-}}{d \Omega}=\frac{d \Sigma^{--}}{d \Omega}+\frac{d \Sigma^{-+}}{d \Omega} .
\end{aligned}
$$

The difference between "spin-up" and "spin-down" SANSPOL cross sections yields information on the polarization-dependent nuclear-magnetic and chiral scattering terms (see Sec. V.A). As demonstrated, e.g., by Keller et al. (2000) on an $\mathrm{Fe}_{3} \mathrm{O}_{4}$ glass ceramic, this difference allows one to highlight weak magnetic contributions relative to strong nuclear scattering (or vice versa). Finally, the unpolarized SANS cross section is obtained as (see Sec. II.B.2)

$$
\begin{aligned}
\frac{d \Sigma}{d \Omega} & =\frac{1}{2}\left(\frac{d \Sigma^{+}}{d \Omega}+\frac{d \Sigma^{-}}{d \Omega}\right) \\
& =\frac{1}{2}\left(\frac{d \Sigma^{++}}{d \Omega}+\frac{d \Sigma^{--}}{d \Omega}+\frac{d \Sigma^{+-}}{d \Omega}+\frac{d \Sigma^{-+}}{d \Omega}\right) .
\end{aligned}
$$

We note that both the unpolarized as well as the half-polarized cross sections can be measured directly. For more information on polarized neutron scattering (and on spherical neutron polarimetry), see the classic papers by Halpern and Johnson (1939), Shull, Wollan, and Koehler (1951), Maleev (1961), Izyumov and Maleev (1962), Blume (1963), Maleev, Bar'yakhtar, and Suris (1963), Marshall and Lowde (1968), Moon, Riste, and Koehler (1969), Okorokov, Runov, and Gukasov (1978), Mezei (1986), Tasset (1989), Schärpf and Capellmann (1993), Brown (2006), and Schweizer (2006) and the textbooks by Squires (1978), Lovesey (1984), Williams (1988), and Hicks (1995).

Although most of the magnetic SANS discussion in this review is treated within the elastic approximation, quasielastic and inelastic scattering contributions are relevant for certain aspects of Secs. VII and VIII. See Maleev (1965), Okorokov et al. (1986), and Grigoriev et al. (2015) for a detailed discussion of inelastic SANS.

\section{B. SANS cross sections}

\section{General considerations}

We restrict our attention to the two commonly used scattering geometries with externally applied magnetic field $\mathbf{H}_{0}$ either perpendicular [Fig. 1(a)] or parallel [Fig. 1(b)] to the incoming neutron beam $\mathbf{k}_{0}$. We adopt a Cartesian laboratory coordinate system with corresponding unit vectors $\mathbf{e}_{x}, \mathbf{e}_{y}$, and $\mathbf{e}_{z}$; the field $\mathbf{H}_{0}$ is assumed to always be parallel to $\mathbf{e}_{z}$. For the perpendicular scattering geometry $\left(\mathbf{k}_{0} \perp \mathbf{H}_{0}\right)$, the angle $\theta$ on the two-dimensional detector is then measured between $\mathbf{H}_{0}$ and the momentum-transfer vector $\mathbf{q} \cong\left\{0, q_{y}, q_{z}\right\}=$ $q\{0, \sin \theta, \cos \theta\}$, whereas for $\mathbf{k}_{0} \| \mathbf{H}_{0}, \theta$ is the angle between $\mathbf{e}_{x}$ and $\mathbf{q} \cong\left\{q_{x}, q_{y}, 0\right\}=q\{\cos \theta, \sin \theta, 0\}$.

The discrete atomic structure of condensed matter is generally of no relevance for SANS, such that the magnetization state of the sample can be represented by a continuous magnetization vector field which is defined at each position $\mathbf{r}$ inside the material. ${ }^{1}$ Magnetic SANS is then a consequence of nanoscale variations in both the orientation and/or magnitude of the magnetization:

\footnotetext{
${ }^{1}$ The case of a smooth modulation of localized or itinerant spins on top of a discrete atomic lattice is discussed in Sec. VIII.
} 


$$
\begin{aligned}
\widetilde{\mathbf{M}}(\mathbf{q}) & =\left\{\widetilde{M}_{x}(\mathbf{q}), \widetilde{M}_{y}(\mathbf{q}), \widetilde{M}_{z}(\mathbf{q})\right\} \\
& =\frac{1}{(2 \pi)^{3 / 2}} \int_{-\infty}^{+\infty} \int_{-\infty}^{+\infty} \int_{-\infty}^{+\infty} \mathbf{M}(\mathbf{r}) e^{-i \mathbf{q} \cdot \mathbf{r}} d^{3} \mathbf{r}
\end{aligned}
$$

represents the three-dimensional Fourier transform of the magnetization

$$
\begin{aligned}
\mathbf{M}(\mathbf{r}) & =\left\{M_{x}(\mathbf{r}), M_{y}(\mathbf{r}), M_{z}(\mathbf{r})\right\} \\
& =\frac{1}{(2 \pi)^{3 / 2}} \int_{-\infty}^{+\infty} \int_{-\infty}^{+\infty} \int_{-\infty}^{+\infty} \tilde{\mathbf{M}}(\mathbf{q}) e^{i \mathbf{q} \cdot \mathbf{r}} d^{3} \mathbf{q}
\end{aligned}
$$

where $i^{2}=-1$, and $\mathbf{q}=\left\{q_{x}, q_{y}, q_{z}\right\}$ is the wave vector. With $\mathbf{H}_{0} \| \mathbf{e}_{z}, \widetilde{M}_{z}$ denotes the longitudinal magnetization Fourier coefficient, whereas $\widetilde{M}_{x}$ and $\widetilde{M}_{y}$ are the transversal components, giving rise to so-called spin-misalignment scattering. For many magnetization configurations it turns out that the functions $\widetilde{M}_{x, y, z}$ are real valued, but, for instance, for structures lacking space inversion symmetry they may pick up an imaginary part (Michels et al., 2016); complex-conjugated quantities are marked by superscript asterisks $(*)$. The nuclear SANS cross section, which is due to nanoscale density and/or compositional fluctuations, is characterized by the Fourier transform $\tilde{N}(\mathbf{q})$ of the continuous scattering-length density $N(\mathbf{r})$.

For the understanding of magnetic neutron scattering, the Halpern-Johnson or magnetic-interaction vector

$$
\mathbf{Q}=\hat{\mathbf{q}} \times[\hat{\mathbf{q}} \times \widetilde{\mathbf{M}}(\mathbf{q})]=\hat{\mathbf{q}}[\hat{\mathbf{q}} \cdot \widetilde{\mathbf{M}}(\mathbf{q})]-\widetilde{\mathbf{M}}(\mathbf{q}),
$$

where $\hat{\mathbf{q}}$ is the unit scattering vector, is of utmost importance (Halpern and Johnson, 1939); it is a manifestation of the dipolar origin of magnetic neutron scattering and it emphasizes the fact that only the component of $\mathbf{M}$ which is perpendicular to $\mathbf{q}$ is relevant for magnetic scattering. ${ }^{2}$ For $\mathbf{k}_{0} \perp \mathbf{H}_{0}$ and $\mathbf{k}_{0} \| \mathbf{H}_{0}$, we obtain, respectively,

$$
\begin{gathered}
\mathbf{Q}_{\perp}=\left\{\begin{array}{c}
-\widetilde{M}_{x} \\
-\widetilde{M}_{y} \cos ^{2} \theta+\widetilde{M}_{z} \sin \theta \cos \theta \\
\widetilde{M}_{y} \sin \theta \cos \theta-\widetilde{M}_{z} \sin ^{2} \theta
\end{array}\right\}, \\
\mathbf{Q}_{\|}=\left\{\begin{array}{c}
-\widetilde{M}_{x} \sin ^{2} \theta+\widetilde{M}_{y} \sin \theta \cos \theta \\
\widetilde{M}_{x} \sin \theta \cos \theta-\widetilde{M}_{y} \cos ^{2} \theta \\
-\widetilde{M}_{z}
\end{array}\right\} .
\end{gathered}
$$

By assuming perfect neutron optics and the fact that the incident neutron polarization is along $\mathbf{e}_{z}$, i.e., $\mathbf{P}=\mathbf{e}_{z}$, the elastic non-spin-flip and spin-flip cross sections can be formally written as (Moon, Riste, and Koehler, 1969)

$$
\frac{d \Sigma^{ \pm \pm}}{d \Omega} \sim|\tilde{N}|^{2} \pm\left(\tilde{N} Q_{z}^{*}+\tilde{N}^{*} Q_{z}\right)+\left|Q_{z}\right|^{2}
$$

\footnotetext{
${ }^{2}$ Note that different symbols for the Halpern-Johnson vector such as $\mathbf{M}_{\perp}, \mathbf{Q}_{\perp}, \mathbf{S}_{\perp}$, or $\mathbf{q}$, as in the original paper by Halpern and Johnson (1939), can be found in the literature.
}

$$
\frac{d \Sigma^{ \pm \mp}}{d \Omega} \sim\left|Q_{x}\right|^{2}+\left|Q_{y}\right|^{2} \mp i\left(Q_{x} Q_{y}^{*}-Q_{x}^{*} Q_{y}\right) .
$$

Several comments are required (Blume, 1963): It is seen that the transversal components $Q_{x}$ and $Q_{y}$ give rise to spin-flip scattering, while the longitudinal component $Q_{z}$ results in non-spin-flip scattering. We also note that the nuclear coherent scattering, the nuclear incoherent scattering which is due to isotope disorder, as well as $1 / 3$ of the nuclear-spin incoherent scattering are all non-spin-flip scattering; the remaining $2 / 3$ of the nuclear-spin incoherent scattering reverses the neutron spin, but, since its magnitude is usually small relative to the coherent magnetic and nuclear SANS of nonhydrogenated samples relevant here (Stuhrmann, 2004) and since it only gives rise to a constant $q$-independent scattering contribution, we ignore it in the spin-resolved channels. Furthermore, if we set $\theta=0^{\circ}$ in Eq. (8), which corresponds to the case that $\mathbf{q} \| \mathbf{P}$, we see that $\mathbf{Q}_{\perp}=\left\{-\widetilde{M}_{x},-\widetilde{M}_{y}, 0\right\}$, so that nuclear coherent and magnetic scattering are fully separated. In the case $\mathbf{k}_{0} \| \mathbf{H}_{0}$ [Eq. (9)], spin-flip scattering probes only the transversal magnetization components $\widetilde{M}_{x, y}$.

In the equations for the cross sections that follow, $V$ denotes the scattering volume, $K=8 \pi^{3} V^{-1} b_{H}^{2}$, where $b_{H}=2.70 \times$ $10^{-15} \mathrm{~m} \mu_{B}^{-1}=2.91 \times 10^{8} \mathrm{~A}^{-1} \mathrm{~m}^{-1}$ is a constant (with $\mu_{B}$ the Bohr magneton), which relates the atomic magnetic moment $\mu_{a}$ to the atomic magnetic scattering length $b_{m}$ (Moon, Riste, and Koehler, 1969):

$$
b_{m}=\frac{\gamma_{n} r_{0}}{2} \frac{\mu_{a}}{\mu_{B}} f(\mathbf{q}) \cong 2.70 \times 10^{-15} m \frac{\mu_{a}}{\mu_{B}} f(\mathbf{q}) \cong b_{H} \mu_{a}
$$

where $\gamma_{n}=1.913$ denotes the neutron magnetic moment expressed in units of the nuclear magneton, $r_{0}=2.818 \times$ $10^{-15} \mathrm{~m}$ is the classical radius of the electron, and $f(\mathbf{q})$ is the normalized atomic magnetic form factor; note that $f \cong 1$ along the forward direction. By inserting Eqs. (8) and (9) into the expressions for the non-spin-flip and spin-flip cross sections, Eqs. (10) and (11), and by noting the relations between the various cross sections, Eqs. (3) and (4), one can conveniently express the SANS cross sections in terms of the Cartesian Fourier components $\widetilde{M}_{x, y, z}$ of the magnetization. We use the following abbreviations for the magnetic and nuclearmagnetic interference terms (subscripts $\perp$ and $\|$ refer to the respective scattering geometry): $C T_{y z}=\widetilde{M}_{y} \widetilde{M}_{z}^{*}+\widetilde{M}_{y}^{*} \widetilde{M}_{z}$, $C T_{x y}=\widetilde{M}_{x} \widetilde{M}_{y}^{*}+\widetilde{M}_{x}^{*} \widetilde{M}_{y}, \quad C T_{\tilde{N} \widetilde{M}_{z}}=\tilde{N} \widetilde{M}_{z}^{*}+\tilde{N}^{*} \widetilde{M}_{z}, \quad$ and $C T_{\tilde{N} \widetilde{M}_{y}}=\tilde{N} \widetilde{M}_{y}^{*}+\tilde{N}^{*} \widetilde{M}_{y}$.

In actual SANSPOL and POLARIS experiments the neutron optics do not work perfectly and polarization corrections become necessary. The incident-beam polarization efficiency is denoted by $P=I^{+} /\left(I^{+}+I^{-}\right)$, where $I^{ \pm}$are, respectively, the number of neutrons with spins aligned antiparallel and parallel with respect to $\mathbf{H}_{0}$ and $\epsilon^{ \pm}$is the efficiency of the spin flipper $\left(\epsilon^{+}=0\right.$ for the flipper off and $\epsilon^{-}=\epsilon \cong 1$ for the flipper on); note that $P=1 / 2$ for an unpolarized beam. The half-polarized SANS cross sections can be obtained directly and corrected for nonideal neutron polarization provided that the parameters $P$ and $\epsilon^{-}$are known from 
reference measurements. For the spin-resolved (POLARIS) cross sections, it is necessary to measure all four partial cross sections in order to correct for spin leakage between the different channels (Wildes, 2006). Such corrections can, for example, be accomplished by means of the BERSANS (Keiderling, 2002; Keiderling et al., 2008), POL-CORR (K. Krycka et al., 2012), and $\mathrm{GRAS}_{\mathrm{ANS}} \mathrm{P}$ (Dewhurst, 2016) software tools. The relevant expressions are as follows.

\section{Unpolarized SANS}

$$
\begin{aligned}
\frac{d \Sigma_{\perp}}{d \Omega}= & K\left(b_{H}^{-2}|\tilde{N}|^{2}+\left|\widetilde{M}_{x}\right|^{2}+\left|\widetilde{M}_{y}\right|^{2} \cos ^{2} \theta\right. \\
& \left.+\left|\widetilde{M}_{z}\right|^{2} \sin ^{2} \theta-C T_{y z} \sin \theta \cos \theta\right), \\
\frac{d \Sigma_{\|}}{d \Omega}= & K\left(b_{H}^{-2}|\tilde{N}|^{2}+\left|\widetilde{M}_{x}\right|^{2} \sin ^{2} \theta+\left|\widetilde{M}_{y}\right|^{2} \cos ^{2} \theta\right. \\
& \left.+\left|\widetilde{M}_{z}\right|^{2}-C T_{x y} \sin \theta \cos \theta\right) .
\end{aligned}
$$

\section{Half-polarized SANS (SANSPOL)}

$$
\begin{aligned}
\frac{d \Sigma_{\perp}^{ \pm}}{d \Omega}= & K\left[b_{H}^{-2}|\tilde{N}|^{2}+\left|\widetilde{M}_{x}\right|^{2}+\left|\widetilde{M}_{y}\right|^{2} \cos ^{2} \theta\right. \\
& +\left|\widetilde{M}_{z}\right|^{2} \sin ^{2} \theta-C T_{y z} \sin \theta \cos \theta \\
& +(2 P-1)\left(2 \epsilon^{ \pm}-1\right) b_{H}^{-1} C T_{\tilde{N} \widetilde{M}_{z}} \sin ^{2} \theta \\
& -(2 P-1)\left(2 \epsilon^{ \pm}-1\right) b_{H}^{-1} C T_{\tilde{N} \widetilde{M}_{y}} \sin \theta \cos \theta \\
& \left.+i(2 P-1)\left(2 \epsilon^{ \pm}-1\right) \chi\right],
\end{aligned}
$$

where the chiral function $\chi$ is given $\mathrm{by}^{3}$

$$
\begin{gathered}
\chi(\mathbf{q})=\left(\widetilde{M}_{x} \widetilde{M}_{y}^{*}-\widetilde{M}_{x}^{*} \widetilde{M}_{y}\right) \cos ^{2} \theta \\
-\left(\widetilde{M}_{x} \widetilde{M}_{z}^{*}-\widetilde{M}_{x}^{*} \widetilde{M}_{z}\right) \sin \theta \cos \theta \\
\frac{d \Sigma_{\|}^{ \pm}}{d \Omega}= \\
\quad K\left[b_{H}^{-2}|\tilde{N}|^{2}+\left|\widetilde{M}_{x}\right|^{2} \sin ^{2} \theta+\left|\widetilde{M}_{y}\right|^{2} \cos ^{2} \theta\right. \\
+\left|\widetilde{M}_{z}\right|^{2}-C T_{x y} \sin \theta \cos \theta \\
\left.+(2 P-1)\left(2 \epsilon^{ \pm}-1\right) b_{H}^{-1} C T_{\tilde{N} \tilde{M}_{z}}\right] .
\end{gathered}
$$

Note that $\chi=0$ for $\mathbf{k}_{0} \| \mathbf{H}_{0}$.

\section{Polarized SANS (POLARIS)}

$$
\begin{aligned}
\frac{d \Sigma_{\perp}^{ \pm \pm}}{d \Omega}= & K\left(b_{H}^{-2}|\tilde{N}|^{2}+\left|\widetilde{M}_{y}\right|^{2} \sin ^{2} \theta \cos ^{2} \theta\right. \\
& +\left|\widetilde{M}_{z}\right|^{2} \sin ^{4} \theta-C T_{y z} \sin ^{3} \theta \cos \theta \\
& \left.\mp b_{H}^{-1} C T_{\tilde{N} \tilde{M}_{z}} \sin ^{2} \theta \pm b_{H}^{-1} C T_{\tilde{N} \widetilde{M}_{y}} \sin \theta \cos \theta\right),
\end{aligned}
$$

\footnotetext{
${ }^{3}$ Note that in the neutron diffraction community the chiral term is sometimes denoted with the symbol " $C$."
}

$$
\begin{aligned}
\frac{d \Sigma_{\perp}^{ \pm \mp}}{d \Omega}= & K\left(\left|\widetilde{M}_{x}\right|^{2}+\left|\widetilde{M}_{y}\right|^{2} \cos ^{4} \theta+\left|\widetilde{M}_{z}\right|^{2} \sin ^{2} \theta \cos ^{2} \theta\right. \\
& \left.-C T_{y z} \sin \theta \cos ^{3} \theta \mp i \chi\right) \\
& \frac{d \Sigma_{\|}^{ \pm \pm}}{d \Omega}=K\left(b_{H}^{-2}|\tilde{N}|^{2}+\left|\widetilde{M}_{z}\right|^{2} \mp b_{H}^{-1} C T_{\tilde{N} \widetilde{M}_{z}}\right), \\
\frac{d \Sigma_{\|}^{ \pm \mp}}{d \Omega}= & K\left(\left|\widetilde{M}_{x}\right|^{2} \sin ^{2} \theta+\left|\widetilde{M}_{y}\right|^{2} \cos ^{2} \theta-C T_{x y} \sin \theta \cos \theta\right) .
\end{aligned}
$$

We reemphasize that the nuclear-spin incoherent scattering is ignored in the spin-flip cross sections.

\section{Magnetic SANS theory}

The expressions given for the SANS cross sections depend on the Fourier components $\widetilde{M}_{x, y, z}$ of the magnetization. The main task is to derive expressions for these functions based on a particular microstructural model. In this section we briefly summarize the recent developments regarding the analytical computation of the cross sections using the theory of micromagnetics (Honecker and Michels, 2013; Metlov and Michels, 2015; Mettus and Michels, 2015; Metlov and Michels, 2016; Michels et al., 2016). Micromagnetics is a phenomenological continuum theory which has been developed in order to compute the magnetization vector field $\mathbf{M}$ of an arbitrarily shaped ferromagnetic body, provided that the applied magnetic field, the geometry of the ferromagnet, and the magnetic material's parameters are known (Brown, 1963; Aharoni, 1996; Kronmüller and Fähnle, 2003). The characteristic length scale which is addressed by micromagnetic calculations ranges between a few nanometers and a few hundreds of nanometers - a size regime that overlaps with the resolution range of the SANS technique. Pioneering work in this direction was performed by Kronmüller, Seeger, and Wilkens (1963) who calculated the magnetic SANS due to spin disorder related to the strain fields of dislocations.

In an attempt to describe the magnetic SANS of a polycrystalline magnetic material, Michels et al. (2016) considered the magnetization response to a spatially varying local saturation magnetization $M_{s}=M_{s}(\mathbf{r})$ and magnetic anisotropy field $\mathbf{H}_{p}=\mathbf{H}_{p}(\mathbf{r})$. Spatial variations in $M_{s}$, e.g., at internal phase boundaries, give rise to magnetostatic stray fields which in turn result in a nanoscale magnetization nonuniformity (representing a contrast for magnetic SANS). Likewise, the field $\mathbf{H}_{p}(\mathbf{r})$ is a source of spin disorder, since it increases the magnitude of the transversal magnetization components. The static equations of micromagnetics for the bulk can be conveniently written as (Brown, 1963; Aharoni, 1996; Kronmüller and Fähnle, 2003)

$$
\mathbf{M}(\mathbf{r}) \times \mathbf{H}_{\mathrm{eff}}(\mathbf{r})=0 .
$$

Equation (21) expresses the fact that at static equilibrium the torque on the magnetization $\mathbf{M}(\mathbf{r})$ due to an effective magnetic field $\mathbf{H}_{\text {eff }}(\mathbf{r})$ vanishes everywhere inside the material. The effective field, 
(a) $\mu_{0} H_{i}=0.02 \mathrm{~T}$

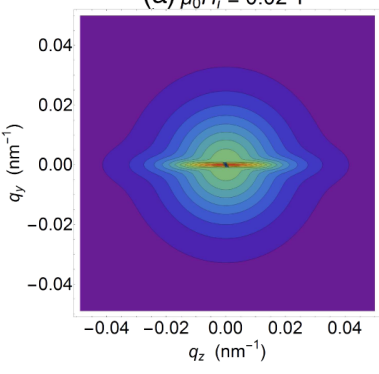

(e) $\mu_{0} H_{i}=0.02 \mathrm{~T}$

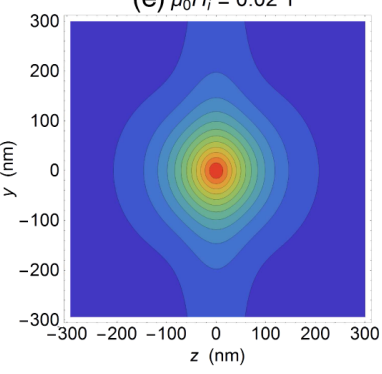

(b) $\mu_{0} H_{i}=0.15 \mathrm{~T}$

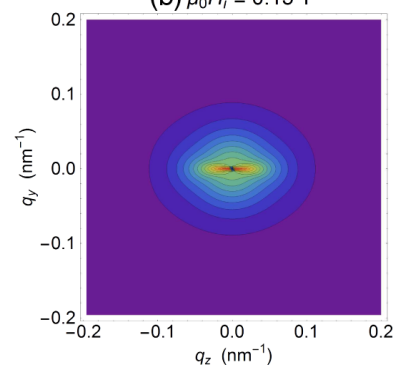

(f) $\mu_{0} H_{i}=0.15 \mathrm{~T}$

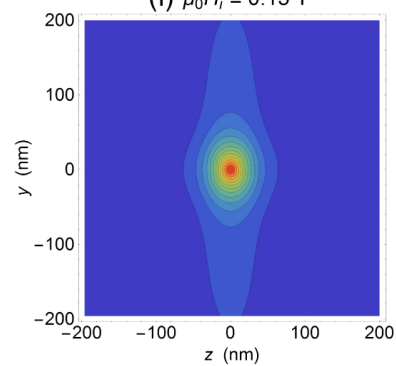

(c) $\mu_{0} H_{i}=1.2 \mathrm{~T}$

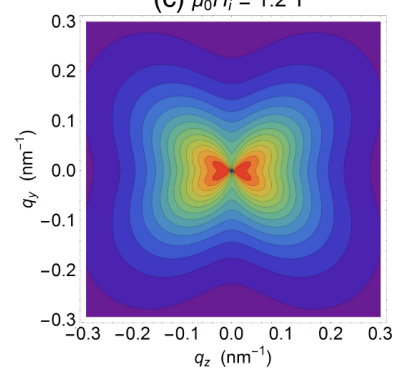

(g) $\mu_{0} H_{i}=1.2 \mathrm{~T}$

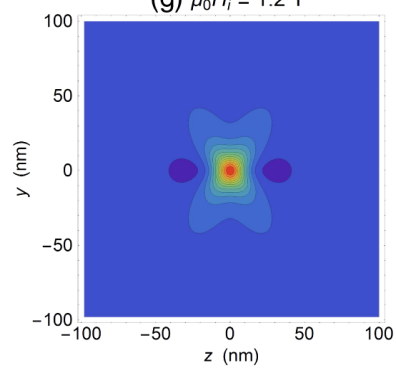

(d) $\mu_{0} H_{i}=11 \mathrm{~T}$

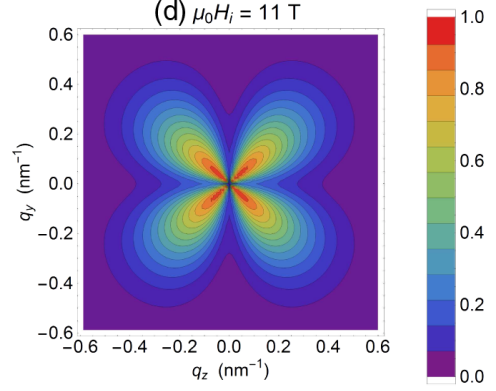

(h) $\mu_{0} H_{i}=11 \mathrm{~T}$

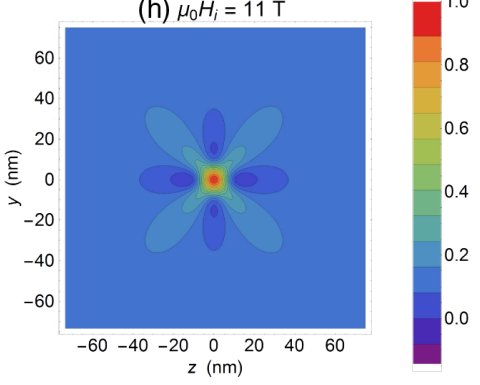

FIG. 2. (a)-(d) Contour plots of normalized $d \Sigma_{M} / d \Omega$ at applied magnetic fields as indicated $\left(\mathbf{k}_{0} \perp \mathbf{H}_{0} ; \mathbf{H}_{0}\right.$ is horizontal); the scattering of the saturated state has been subtracted. (e)-(h) Corresponding two-dimensional correlation functions $c(y, z)$ [ $=2 \mathrm{D}$ Fourier transforms of $\left.d \Sigma_{M}\left(q_{y}, q_{z}\right) / d \Omega\right]$. The DMI has not been taken into account. From Mettus and Michels, 2015.

$$
\mathbf{H}_{\mathrm{eff}}=\mathbf{H}_{0}+\mathbf{H}_{d}+\mathbf{H}_{p}+\mathbf{H}_{e x}+\mathbf{H}_{\mathrm{DM}}
$$

is composed of a uniform applied magnetic field $\mathbf{H}_{0}$, the magnetostatic field $\mathbf{H}_{d}(\mathbf{r})$, the magnetic anisotropy field $\mathbf{H}_{p}(\mathbf{r})$, the exchange field $\mathbf{H}_{e x}$, and the field $\mathbf{H}_{\mathrm{DM}}$, which is due to the Dzyaloshinski-Moriya interaction (DMI). In the approach-to-saturation regime, when the sample consists of a single magnetic domain and small spin deviations from the mean magnetization are considered $\left(M_{x} \ll M_{s}\right.$ and $M_{y} \ll M_{s} ; \mathbf{M}_{s} \| \mathbf{e}_{z}$ ), the balance-of-torques equation can be linearized and a closed-form solution for the transversal Fourier components can be found (Michels et al., 2016); these allow one to compute any magnetic SANS cross section.

As an example, Fig. 2 displays for $\mathbf{k}_{0} \perp \mathbf{H}_{0}$ and an unpolarized beam the spin-misalignment SANS cross section $d \Sigma_{M} / d \Omega$ arising due to transversal magnetization components. The term spin-misalignment SANS cross section refers to the cross section which remains at a particular value of the applied magnetic field when the total (nuclear and magnetic) $d \Sigma / d \Omega$ in the saturated state is subtracted [compare Eq. (23)]. At the largest fields [Figs. 2(c) and 2(d)], $d \Sigma_{M} / d \Omega$ exhibits maxima roughly along the diagonals of the detector- the socalled "clover-leaf" anisotropy-previously observed in the Fe-based two-phase alloy NANOPERM [compare, e.g., Fig. 3 in Michels et al. (2006)]. The positions of the maxima in $d \Sigma_{M} / d \Omega$ depend on $q$ and $H_{0}$ [see also Fig. 11 in Michels et al. (2014)]. Reducing the field [Figs. 2(a) and 2(b)], one observes an elongation of the spin-misalignment scattering along the field direction, with a "flying-saucer-type" pattern taking over at small $q$ and $H_{0}$. The sharp spike in Fig. 2(a) is due to the magnetostatic interaction; it was first predicted by Weissmüller et al. (1999) and experimentally observed by Périgo et al. (2014) on a Nd-Fe-B-based permanent magnet (see Fig. 3).
The corresponding two-dimensional correlation functions $c(y, z)$ are displayed in Figs. 2(e)-2(h) at the same fields as $d \Sigma_{M} / d \Omega$ (Mettus and Michels, 2015). While $d \Sigma_{M} / d \Omega$ at small fields [Figs. 2(a) and 2(b)] is enhanced parallel to $\mathbf{H}_{0}$, the correlation function exhibits maxima in the direction perpendicular to $\mathbf{H}_{0}$; the range of the correlations extends to several hundreds of nanometers [Figs. 2(e) and 2(f)]. Increasing the field results in the suppression of the correlations and at the largest field $d \Sigma_{M} / d \Omega$ possesses a nearly fourfold anisotropy with maxima along the detector diagonals and minima along the horizontal and vertical axes [Fig. 2(d)], which translate into the corresponding extrema in $c(y, z)$ [Fig. 2(h)]. In nuclear SANS, negative values of the distance distribution function $p(r)$ are attributed to distances that connect regions with opposite sign of the scattering-length density contrast more frequently than regions with the same sign (Glatter and Kratky, 1982). However, for magnetic SANS, such an easily accessible interpretation of the correlation function of the spin-misalignment SANS cross section
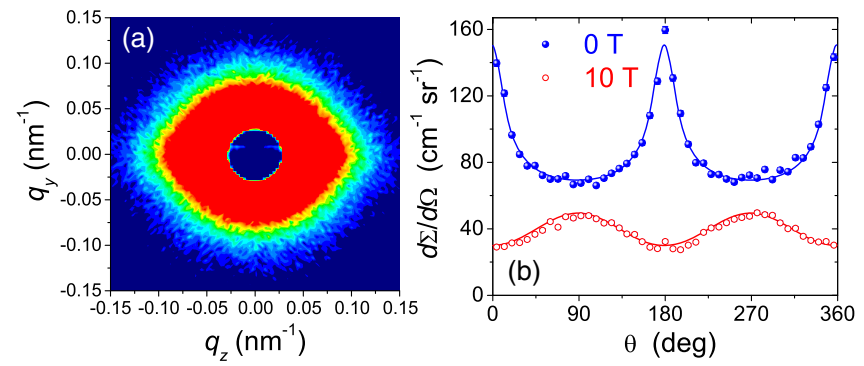

FIG. 3. (a) Spike anisotropy observed in the total unpolarized $d \Sigma / d \Omega$ of a sintered $\mathrm{Nd}$-Fe-B-based permanent magnet in the remanent state $\left(\mathbf{k}_{0} \perp \mathbf{H}_{0}\right)$. (b) $d \Sigma / d \Omega$ vs azimuthal angle $\theta$ at $q=0.10 \pm 0.02 \mathrm{~nm}^{-1}$. From Périgo et al., 2014 . 
in terms of a specific magnetization distribution is not straightforward. This is mainly related to the fact that $c(y, z)$ - being the Fourier transform of $d \Sigma_{M} / d \Omega$ - does not directly represent the correlations in the magnetic microstructure (as does the autocorrelation function), but also includes the magnetodipolar interaction of the neutrons with the sample (via the trigonometric functions and the cross term in the cross section) (Erokhin, Berkov, and Michels, 2015).

It was recently suggested that the DMI is of relevance for the magnetic SANS of materials containing many lattice imperfections (Michels et al., 2016), e.g., due to the breaking of inversion symmetry at internal interfaces, which may cause a chiral term, Eq. (15). The defect-induced symmetry breaking can be characterized from measurement of the spin-flip cross sections, Eq. (18), according to

$$
-2 i \chi(\mathbf{q})=\frac{d \Sigma^{+-}}{d \Omega}-\frac{d \Sigma^{-+}}{d \Omega} .
$$

Using the expressions for the Fourier components (Michels et al., 2016), the function $-2 i \chi(\mathbf{q})$ is plotted in Fig. 4. At small fields, two extrema parallel and antiparallel to the field axis are predicted [Fig. 4(a)], whereas at larger fields additional maxima and minima appear approximately along the detector diagonals [Figs. 4(b) and 4(c)]. The strong field dependency of $-2 i \chi\left(q, H_{0}\right)$ may be employed in order to experimentally determine the DM constant (Michels et al., 2019). Note that $-2 i \chi(\mathbf{q})$ describes an asymmetry arising in the elastic SANS cross section due to the effect of the antisymmetric exchange interaction on the static magnetic microstructure. See Sec. VIII for further studies which address the inelastic and critical scattering related to the DM term.

As shown by Honecker et al. (2013), near magnetic saturation and for a two-phase particle-matrix-type ferromagnet, the unpolarized $d \Sigma / d \Omega$ for $\mathbf{k}_{0} \perp \mathbf{H}_{0}$ can be evaluated by means of micromagnetic theory. As an example, the azimuthally averaged field-dependent SANS cross section of a zeromagnetostriction nanocomposite from the NANOPERM family of alloys along with the fits to the micromagnetic theory is displayed in Fig. 5(a). It is seen that the entire $\left(q, H_{0}\right)$ dependence of $d \Sigma / d \Omega$ can be well described by the micromagnetic prediction. From a global fit of the entire data set to the micromagnetic theory one obtains a value of $A=4.7 \pm$ $0.9 \mathrm{pJ} / \mathrm{m}$ for the volume-averaged exchange-stiffness constant [compare inset in Fig. 5(a)].

In addition to the exchange constant, the analysis provides the square magnitudes of the Fourier coefficients of the magnetic anisotropy field $S_{H} \propto\left|\tilde{\mathbf{H}}_{p}(q)\right|^{2}$ and of the longitudinal
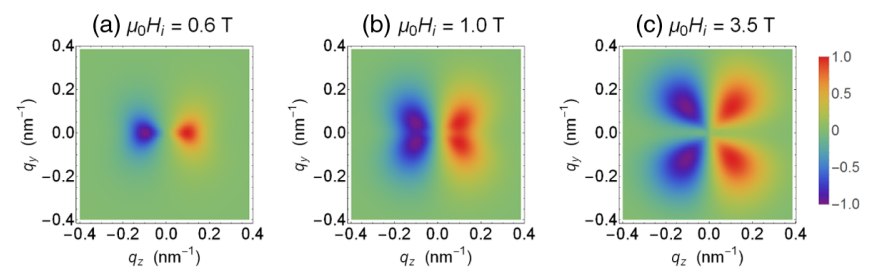

FIG. 4. Contour plots of the spin-flip difference cross section $-2 i \chi(\mathbf{q})$ at selected applied magnetic fields $\left(\mathbf{k}_{0} \perp \mathbf{H}_{0}\right)$. From Michels et al., 2016.
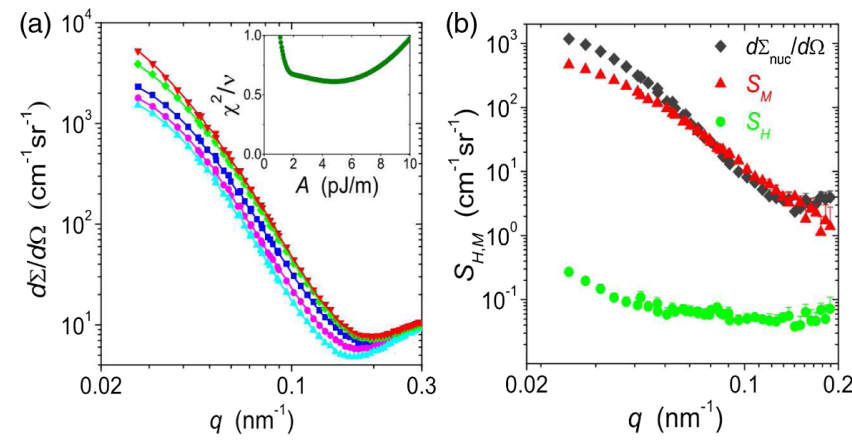

FIG. 5. (a) Azimuthally averaged $d \Sigma / d \Omega$ of the two-phase alloy $\left(\mathrm{Fe}_{0.985} \mathrm{Co}_{0.015}\right)_{90} \mathrm{Zr}_{7} \mathrm{~B}_{3}$ at selected applied magnetic fields (log$\log$ scale). Field values (in $\mathrm{mT}$ ) from bottom to top: 1270, 312, 103, 61, and 33. Solid lines: fits to the micromagnetic theory. The inset depicts the mean-square deviation between experiment and fit, $\chi^{2} / \nu$, as a function of the exchange-stiffness constant $A$. (b) Best-fit results for the scattering function of the anisotropy field $S_{H} \propto\left|\tilde{H}_{p}(q)\right|^{2}$ and for the scattering function of the longitudinal magnetization $S_{M} \propto\left|\widetilde{M}_{z}(q)\right|^{2} . d \Sigma_{\text {nuc }} / d \Omega$ denotes the nuclear SANS (log-log scale). From Honecker et al., 2013.

magnetization $S_{M} \propto\left|\widetilde{M}_{z}(q)\right|^{2} \propto(\Delta M)^{2}$. The results obtained for these functions are shown in Fig. 5(b). It is immediately seen that over the displayed $q$ range $\left|\widetilde{M}_{z}\right|^{2}$ is orders of magnitude larger than $\left|\tilde{H}_{p}\right|^{2}$, suggesting that jumps $\Delta M$ in the magnetization at internal interfaces are the dominating source of spin disorder in these alloys. Numerical integration of $S_{H}(q)$ and $S_{M}(q)$ over the whole $\mathbf{q}$ space, i.e., $\left(2 \pi^{2} b_{H}^{2}\right)^{-1} \int_{0}^{\infty} S_{H, M} q^{2} d q$ yields, respectively, the mean-square anisotropy field $\left\langle\left|\mathbf{H}_{p}\right|^{2}\right\rangle$ and the mean-square longitudinal magnetization fluctuation $\left\langle\left|M_{z}\right|^{2}\right\rangle$ (Honecker et al., 2013). For the data shown in Fig. 5(b) we obtain the following lower bounds: $\mu_{0}\left\langle\left|\mathbf{H}_{p}\right|^{2}\right\rangle^{1 / 2} \cong 10 \mathrm{mT}$ and $\mu_{0}\left\langle\left|M_{z}\right|^{2}\right\rangle^{1 / 2} \cong 50 \mathrm{mT}$. This finding qualitatively supports the notion of dominant spin-misalignment scattering due to magnetostatic fluctuations.

\section{Relation to conventional particle-matrix approach}

Magnetic SANS of bulk magnetic materials (e.g., singlephase elemental ferromagnets, $\mathrm{Nd}-\mathrm{Fe}-\mathrm{B}$-based permanent magnets, or steels) is to a large extent determined by longrange magnetization fluctuations due to defect-related spin misalignment. Away from magnetic saturation, all three magnetization Fourier components $\widetilde{\mathbf{M}}=\left\{\widetilde{M}_{x}, \widetilde{M}_{y}, \widetilde{M}_{z}\right\}$ govern the magnetic SANS cross sections, whereas in the saturated state, when $\mathbf{M}=\left\{0,0, M_{z}=M_{\mathrm{s}}(\mathbf{r})\right\}$, the cross sections are determined by nuclear SANS and by the Fourier transform $\widetilde{M}_{\mathrm{s}}(\mathbf{q})$ of the spatially dependent saturation magnetization $M_{s}(\mathbf{r})$. It is important to realize that the spinmisalignment SANS cross section depends primarily on the magnetic interactions (but also on the underlying grain microstructure), while the SANS at saturation is entirely determined by the geometry of the microstructure, in other words, $|\tilde{N}|^{2}$ and $\left|\widetilde{M}_{s}\right|^{2}$ depend only on the size, shape, position of particles, and the scattering-length density contrast of the particles relative to the matrix. For instance, for the 
perpendicular and parallel scattering geometry, the unpolarized cross sections at saturation reduce, respectively, to

$$
\frac{d \Sigma_{\perp}^{\mathrm{sat}}}{d \Omega}=K\left(b_{H}^{-2}|\tilde{N}|^{2}+\left|\widetilde{M}_{s}\right|^{2} \sin ^{2} \theta\right)
$$

and

$$
\frac{d \Sigma_{\|}^{\mathrm{sat}}}{d \Omega}=K\left(b_{H}^{-2}|\tilde{N}|^{2}+\left|\widetilde{M}_{s}\right|^{2}\right) .
$$

The magnetic structure factor of a saturated microstructure consisting of a distribution of $i=1, \ldots, N_{p}$ particles with saturation magnetization $M_{s, i}^{p}$ in a matrix of saturation magnetization $M_{s}^{m}$ can be written as (Schlömann, 1967)

$$
\begin{aligned}
\left|\widetilde{M}_{s}(\mathbf{q})\right|^{2}= & \frac{1}{8 \pi^{3}} \sum_{i=1}^{N_{p}} \sum_{j=1}^{N_{p}}\left(M_{s}^{m}-M_{s, i}^{p}\right)\left(M_{s}^{m}-M_{s, j}^{p}\right) \\
& \times V_{p, i} V_{p, j} F_{i}(\mathbf{q}) F_{j}^{*}(\mathbf{q}) e^{-i \mathbf{q} \cdot\left(\mathbf{r}_{i}-\mathbf{r}_{j}\right)}
\end{aligned}
$$

where $V_{p, i}, F_{i}$, and $\mathbf{r}_{i}$ represent, respectively, the particle volume, the form factor, and the position vector of particle " $i$." An analogous expression describes the corresponding nuclear SANS [see, e.g., Chen and Lin (1987)].

In the monodisperse and dilute limit, we have

$$
\left|\widetilde{M}_{s}(\mathbf{q})\right|^{2}=\frac{N_{p}}{8 \pi^{3}}(\Delta M)^{2} V_{p}^{2}|F(\mathbf{q})|^{2},
$$

where $\Delta M=M_{s}^{m}-M_{s}^{p}$. Inserting Eq. (26) into Eqs. (23) and (24), the magnetic SANS cross section at saturation (e.g., for $\mathbf{k}_{0} \perp \mathbf{H}_{0}$ ) takes on the familiar form (Wiedenmann, 2001)

$$
\frac{d \Sigma_{\perp}^{\mathrm{sat}}}{d \Omega}=\frac{N_{p}}{V} \Delta \eta_{m}^{2} V_{p}^{2}|F(\mathbf{q})|^{2} \sin ^{2} \theta,
$$

where $\Delta \eta_{m}^{2}=b_{H}^{2}(\Delta M)^{2}$ denotes the magnetic scatteringlength density contrast. Equation (27) represents the wellknown expression - embodying the particle-matrix conceptwhich is employed in many magnetic SANS investigations, even in situations where the material under study is not fully saturated. As the derivation has shown, Eq. (27) relies on the special assumption of homogeneously magnetized domains $\left(M_{x}=M_{y}=0\right)$, and for various reasons [see, e.g., the discussion in Michels (2014)] Eq. (27) does not describe the magnetic SANS of bulk ferromagnets (unless fully saturated). However, as discussed in Sec. V, the magnetic SANS of nearly uniformly magnetized nanoparticles may be described by means of the particle-matrix approach by employing special profiles for the magnetic scattering-length densities (Disch et al., 2012).

Analytical expressions for particle form factors $|F(\mathbf{q})|^{2}$ have been derived for an extensive number of particle shapes and there exist also a few closed-form results for the structure factor (Guinier and Fournet, 1955; Glatter and Kratky, 1982; Feigin and Svergun, 1987; Pedersen, 1997). See Pedersen (1997) for a detailed discussion of this topic. Likewise, several software packages (Kline, 2006; Breßler, Kohlbrecher, and
Thünemann, 2015; Butler et al., 2016) provide collections of particle form-factor models (including particle-size distributions) and structure factors to analyze SANS data.

Before closing this section we briefly introduce two important limiting expressions for the scattering curve, which allows one to obtain information about the structure size and the internal particle surface area. Generally, when there are two phases of uniform scattering-length density and with discontinuous (sharp) interfaces, the scattering in the limit of large $q$ (much larger than the inverse of the characteristic structure scale) obeys (Debye and Bueche, 1949; Debye, Anderson, and Brumberger, 1957; Porod, 1982a; Ciccariello, Goodisman, and Brumberger, 1988)

$$
\frac{d \Sigma}{d \Omega}(q) \cong 2 \pi(\Delta \eta)^{2} \frac{S}{V} q^{-4},
$$

where $S$ denotes the particle surface area. Equation (28) is known as the Debye-Porod law, and it is valid not only for single particles, but also for densely packed systems. This expression for the high- $q$ limit can be supplemented by one for the scattering near the origin of reciprocal space, the socalled Guinier approximation (Guinier, 1994): when the scattering is from a set of noninterfering discrete objects then, in the limit of low $q<1.3 / R_{g}$,

$$
\frac{d \Sigma}{d \Omega}(q) \cong \frac{d \Sigma}{d \Omega}(0) e^{-q^{2} R_{g}^{2} / 3} .
$$

For identical scatterers, $R_{g}$ denotes the individual radius of gyration. In fact, for dilute monodisperse systems, the Guinier plot $\left[\ln (d \Sigma / d \Omega)\right.$ vs $\left.q^{2}\right]$ should be a linear function whose slope yields $R_{g}$. As pointed out by Svergun and Koch (2003), linearity of the Guinier plot can be considered as a test of the sample homogeneity and deviations indicate attractive or repulsive interparticle interactions leading to interference effects (Rothwell, 1968). Furthermore, when the particle-size distribution is nonuniform, $R_{g}^{2}$ needs to be replaced with the ratio of moments of the size distribution [see Kostorz (1982) and Feigin and Svergun (1987) for details].

The Porod and Guinier laws have been derived for nonmagnetic particle-matrix-type assemblies in the context of the early theoretical developments of the technique of small-angle $\mathrm{x}$-ray scattering and their application to magnetic materials should be considered with special care. They are certainly applicable to systems consisting of saturated magnetic particles in a nonmagnetic matrix or, likewise, to pores in a saturated matrix. On the other hand, when the smoothly varying magnetization profiles of micromagnetics (Sec. VI) are at the origin of the related magnetic scattering, implying the absence of a sharp interface in the magnetic microstructure, the asymptotic power-law behavior of the cross section differs from the $q^{-4}$ behavior [see, e.g., Fig. 7(b)], in agreement with theoretical predictions and experimental observations (Mettus and Michels, 2015). This statement does not, of course, preclude the existence of sharp interfaces in the nuclear grain microstructure of a magnetic material: there may well exist sharp particle-matrix interfaces, but the corresponding spin distribution which decorates these 
interfaces and which gives rise to the magnetic SANS cross section might be continuous over the defects.

\section{Nd-Fe-B-BASED PERMANENT MAGNETS}

This section addresses the properties of Nd-Fe-B-based permanent magnets as seen by magnetic SANS. The major challenge in this field remains the understanding of how the details of the microstructure (e.g., average grain size and shape, distribution of Nd-rich intergranular phases, crystallographic texture, or interfacial chemistry) correlate with the macroscopic magnetic properties. In order to tackle this issue, a multiscale characterization approach is generally adopted, which comprises a suite of both experimental and theoretical state-of-the-art methods including high-resolution electron microscopy, electron backscattering diffraction, threedimensional atom-probe analysis, Lorentz and Kerr microscopy, or atomistic and continuum micromagnetic simulations (Gutfleisch et al., 2011; Hono and Sepehri-Amin, 2012; Woodcock et al., 2012; Liu et al., 2013; Sepehri-Amin et al., 2013, 2015). The SANS technique has made an important contribution here, since it provides, quite uniquely, information on variations of both the magnitude and orientation of the magnetization vector on a nanometer length scale and from within the volume of the material. We begin the discussion in this section by introducing the concept of the correlation function of the spin-misalignment SANS cross section and we then discuss selected SANS results obtained on Ne-Fe-B-based nanocomposites and sintered magnets.

\section{A. Correlation function of the spin-misalignment SANS cross section}

Using azimuthally averaged data for the spin-misalignment SANS cross section $d \Sigma_{M} / d \Omega$ it becomes possible to compute the correlation function $C(r)$ of the spin-misalignment SANS cross section, according to (Mettus and Michels, 2015)

$$
C(r) \sim \int_{0}^{\infty} \frac{d \Sigma_{M}}{d \Omega}(q) j_{0}(q r) q^{2} d q,
$$

where $j_{0}(x)=\sin (x) / x$ denotes the zeroth-order spherical Bessel function. By means of the extrapolated value of the correlation function at the origin $C(r=0)$, one can determine the correlation length $l_{C}$ of the spin misalignment. Figure 6 illustrates the meaning of $l_{C}$, which specifies the range over which perturbations in the spin structure around a lattice defect (e.g., pore, grain boundary, dislocation, or vacancy) are transmitted by the exchange interaction into the surrounding crystal lattice. A convenient definition for $l_{C}$ is

$$
C\left(r=l_{C}\right)=C(0) e^{-1},
$$

which yields the exact correlation length for exponentially decaying correlations. Note, however, that this definition does not imply that the correlations do decay exponentially. Equation (31) is merely a convenient way to define a characteristic length which can be related to the magnetic microstructure and which can be computed model independently.

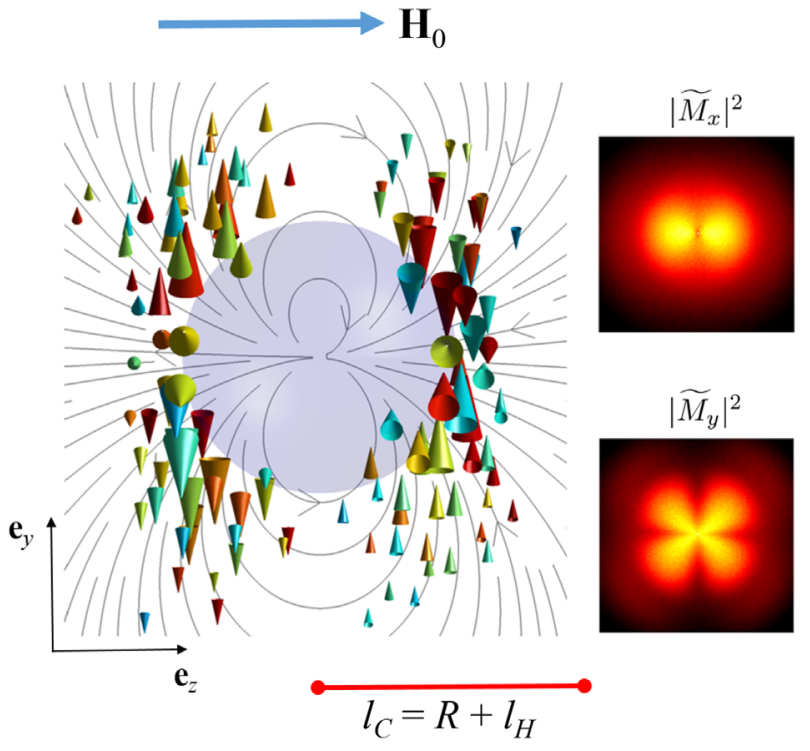

FIG. 6. The correlation length $l_{C}$ of the spin misalignment. (Left) Computed spin misalignment (at $\mu_{0} H_{0}=0.6 \mathrm{~T}$ ) around a spherical pore $(2 R=12 \mathrm{~nm})$ in a ferromagnetic iron matrix (twodimensional cut out of a three-dimensional simulation). Shown is the magnetization component $\mathbf{M}_{\perp}(\mathbf{r})$ perpendicular to $\mathbf{H}_{0} \| \mathbf{e}_{z}$; the thickness of the arrows is proportional to the magnitude of $\mathbf{M}_{\perp}$. Solid gray lines: magnetodipolar field distribution. The correlation length $l_{C}$ is a measure for the size of the inhomogeneously magnetized region around the defect; $l_{C}$ consists of a fieldindependent contribution $R$, which specifies the structural size of the defect, and of a field-dependent exchange length $l_{H}$, which transmits the perturbation at the pore-matrix interface into the surrounding crystal lattice. (Right) Corresponding magnetization Fourier components $\left|\widetilde{M}_{x}(\mathbf{q})\right|^{2}$ and $\left|\widetilde{M}_{y}(\mathbf{q})\right|^{2}$ projected into the plane $q_{x}=0$. The bright colors correspond to "high" values and the dark colors to "low" values of the Fourier components. Pixels in the corners of the images have $q \cong 0.4 \mathrm{~nm}^{-1}$. A logarithmic color scale is used. From Erokhin, Berkov, and Michels, 2015.

In several studies (Michels et al., 2003; Michels and Weissmüller, 2008; Michels, 2010; Bick et al., 2013; Honecker and Michels, 2013) it was found that $l_{C}\left(H_{i}\right)$ data can be well described by

$$
l_{C}\left(H_{i}\right)=R+\sqrt{\frac{2 A}{\mu_{0} M_{s}\left(H_{i}+H^{\star}\right)}},
$$

where the field-independent parameter $R$ is of the order of the defect size and the second field-dependent term on the righthand side represents a modified exchange length $l_{H}$ of the field [see Eq. (33) for the definition of $l_{H}$ ]. Equation (32) is a phenomenological prediction based on micromagnetic theory, which embodies the convolution relationship between the magnetic anisotropy-field microstructure $\mathbf{H}_{p}(\mathbf{r})$ and micromagnetic response functions which decay with $l_{H}$ (Weissmüller et al., 1999, 2001). The "correlation length" $R$ of the magnetic anisotropy field appears to be the average size over which the direction and/or magnitude of $\mathbf{H}_{p}$ changes. For a statistically isotropic polycrystalline material, where each crystallite is a single crystal with magnetocrystalline 
anisotropy only, the parameter $R$ is sensibly related to the average crystallite size (Michels, 2010; Honecker and Michels, 2013). The field $H^{\star}$ is expected to model the influence of the magnetodipolar interaction and the magnetic anisotropy (Bick et al., 2013). For soft magnetic materials with low crystalline anisotropy and at large applied magnetic fields (when the magnetostatic interaction may be negligible), one may ignore the field $H^{\star}$, so that Eq. (32) simplifies to

$$
l_{C}\left(H_{i}\right)=R+l_{H}\left(H_{i}\right)=R+\sqrt{\frac{2 A}{\mu_{0} M_{s} H_{i}}} .
$$

The latter equation has been found to describe well the fielddependent spin-misalignment correlations in nanocrystalline $\mathrm{Co}$ and $\mathrm{Ni}$ (Michels et al., 2003). By contrast, for uniaxial hard magnets, the anisotropy field $H_{K}=2 K_{u} / M_{s}$, which for $\mathrm{Nd}_{2} \mathrm{Fe}_{14} \mathrm{~B}$ single crystal is about $8 \mathrm{~T}$ at $300 \mathrm{~K}$ (Woodcock et al., 2012), is expected to cut down the size of spin inhomogeneities. Likewise, jumps $\Delta M$ of the magnitude of the magnetization at internal phase boundaries, which in Fe-based nanocomposites can be as large as $1.5 \mathrm{~T}$ (Michels et al., 2006), give rise to magnetic stray-field torques that produce spin disorder in the surrounding magnetic phase (compare Fig. 6); such kinds of perturbations also decrease the size of gradients in the magnetization (Honecker et al., 2013). It is interesting to note that at $H_{i}=0$ and for $H^{\star}=H_{K}=2 K_{u} / \mu_{0} M_{s}$, Eq. (32) reduces to

$$
l_{C}=R+\sqrt{\frac{A}{K_{u}}},
$$

where $\sqrt{A / K_{u}}$ is the domain-wall parameter.

\section{B. Selected results on Nd-Fe-B magnets}

A chronological assessment of SANS studies on Nd-Fe-Bbased permanent magnets starts with the early work of Fujii et al. (1987), who investigated the role of domain walls and grain boundaries on the magnetic microstructure of sintered $\mathrm{Nd}_{15} \mathrm{Fe}_{77} \mathrm{~B}_{8}$ and $\mathrm{Nd}_{15} \mathrm{Fe}_{76} \mathrm{Al}_{1} \mathrm{~B}_{8}$. Despite this pioneering approach of using the SANS method for studying rareearth-based intermetallic compounds, only recently (more precisely in the last five years) has a deeper understanding of the capabilities (and limitations) of the SANS technique been reached. It was Takeda et al. (2012) who continued with SANS research on sintered Nd-Fe-B magnets by analyzing the temperature dependence of SANS patterns with special attention to the correlation between the average structure and the coercivity. The effect of the grain-boundary diffusion process on the magnetization reversal of isotropic sintered (Périgo et al., 2016) and hot-deformed (textured) nanocrystalline (Yano et al., 2012, 2014; Saito et al., 2015) Nd-Fe-B magnets has been investigated.

We start by considering a melt-spun isotropic $\mathrm{Nd}-\mathrm{Fe}-\mathrm{B}-$ based nanocomposite, which consists of hard magnetic $\mathrm{Nd}_{2} \mathrm{Fe}_{14} \mathrm{~B}$ particles (size $\sim 22 \mathrm{~nm}$ ) and $\mathrm{Fe}_{3} \mathrm{~B}$ crystallites (size $\sim 29 \mathrm{~nm}$ ) (Bick et al., 2013; Bick, Suzuki et al., 2013). It is important to mention that for this particular alloy the difference
$\Delta M$ in the saturation magnetizations of the $\mathrm{Nd}_{2} \mathrm{Fe}_{14} \mathrm{~B}$ phase and the $\mathrm{Fe}_{3} \mathrm{~B}$ crystallites is rather small, $\mu_{0} \Delta M \cong 0.01 \mathrm{~T}$ (Schrefl, Kronmüller, and Fidler, 1993). Consequently, the related longitudinal magnetic SANS $\propto\left|\widetilde{M}_{z}\right|^{2} \propto(\Delta M)^{2}$ is negligible as compared to the nuclear SANS $|\tilde{N}|^{2}$.

Figure 7(a) displays the total unpolarized $d \Sigma / d \Omega$ of the $\mathrm{Nd}$ Fe-B nanocomposite. A strong field dependence between the largest applied field of $10 \mathrm{~T}$ and the coercive field of $\mu_{0} H_{c}=$ $-0.55 \mathrm{~T}$ is observable. Since nuclear SANS is field independent and since SANS due to $\left|\widetilde{M}_{z}\right|^{2}$ fluctuations is negligible (for this particular alloy), it is evident that the dominating contribution to $d \Sigma / d \Omega$ is due to transversal spin misalignment. In order to obtain the corresponding spin-misalignment SANS cross section [see Fig. 7(b)], the $d \Sigma / d \Omega$ at 10 T was subtracted from the $d \Sigma / d \Omega$ at lower fields. The resulting $d \Sigma_{M} / d \Omega$ is of comparable magnitude as $d \Sigma / d \Omega$, but possesses a strikingly different $q$ dependency. In particular, the shoulder in $d \Sigma / d \Omega$ at
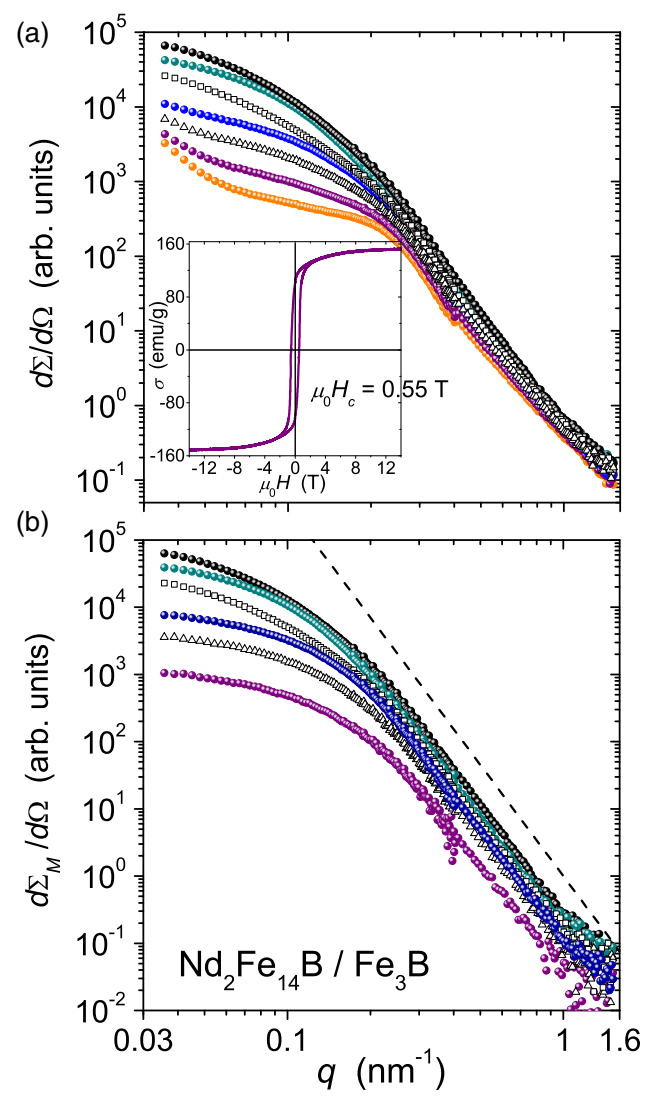

FIG. 7. (a) Azimuthally averaged total SANS cross section $d \Sigma / d \Omega$ of $\mathrm{Nd}_{2} \mathrm{Fe}_{14} \mathrm{~B} / \mathrm{Fe}_{3} \mathrm{~B}$ as a function of momentum transfer $q$ and applied magnetic field $H_{0}(T=300 \mathrm{~K})\left(\mathbf{k}_{0} \perp \mathbf{H}_{0}\right)(\log -\log$ scale). Solid circles: applied-field values (in Tesla) decrease from bottom to top: $10,6,1,-0.25$, and -0.55 ; squares: $-1 \mathrm{~T}$; triangles: $-3 \mathrm{~T}$. Inset: room-temperature magnetization curve of $\mathrm{Nd}_{2} \mathrm{Fe}_{14} \mathrm{~B} / \mathrm{Fe}_{3} \mathrm{~B}$. (b) Applied-field dependence of the spinmisalignment SANS cross section $d \Sigma_{M} / d \Omega$ of nanocrystalline $\mathrm{Nd}_{2} \mathrm{Fe}_{14} \mathrm{~B} / \mathrm{Fe}_{3} \mathrm{~B}$. Solid circles: field values (in Tesla) decrease from bottom to top: $6,1,-0.25$, and -0.55 ; squares: $-1 \mathrm{~T}$; triangles: $-3 \mathrm{~T}$. The $d \Sigma_{M} / d \Omega$ data displayed in (b) were obtained by subtracting the $10 \mathrm{~T}$ data shown in (a) from the $d \Sigma / d \Omega$ at lower fields. Dashed line: $d \Sigma_{M} / d \Omega \propto q^{-5.5}$. From Bick et al., 2013. 
about $q=0.2 \mathrm{~nm}^{-1}$ is absent in $d \Sigma_{M} / d \Omega$. Possible origins for the shoulder in $d \Sigma / d \Omega$ are interparticle interferences and/or diffusion zones around the particles as discussed by Heinemann et al. (2000). The different shapes of $d \Sigma / d \Omega$ and $d \Sigma_{M} / d \Omega$ are also reflected in different asymptotic power-law exponents $n$ in $d \Sigma_{M} / d \Omega \propto q^{-n}$. While the spin-misalignment SANS is characterized by power-law exponents which range between $n \sim 5$ and 6 at all fields investigated [see Fig. 7(b)], the total unpolarized SANS reveals significantly lower values for $n$, which approach the Porod value of $n=4$ at $10 \mathrm{~T}$ (Bick et al., 2013).

Fourier transformation of the $d \Sigma_{M} / d \Omega$ data according to Eq. (30) yields the correlation function $C(r)$ of the spin misalignment (see Fig. 8). The field-dependent correlations in Fig. 8 do not decay exponentially, in agreement with the absence of an $n=4$ power-law exponent in $d \Sigma_{M} / d \Omega$. Furthermore, the $C(r)$ seem to approach the origin $r=0$ with zero slope (compare dotted line in Fig. 8), which is in agreement with the notion of magnetic SANS from continuously varying magnetization profiles and with the absence of a sharp interface in the magnetic microstructure (Porod, 1982a). For comparison, for the example of a uniform sphere (of radius $R$ ) and with a sharp interface, one finds the wellknown expression (valid for $r \leq 2 R$ )

$$
c_{s}(r)=1-\frac{3 r}{4 R}+\frac{r^{3}}{16 R^{3}},
$$

from which one can recognize that the first derivative of $c_{s}(r)$ evaluated at $r=0$ is related to the surface $S$ of the particle $c_{s}^{\prime}(0)=-3 /(4 R)=-S /(4 V)$. Such properties of the correlation function (derived for the nuclear small-angle scattering of uniform particles) do not hold for magnetic SANS of bulk ferromagnets.

The values of the correlation length $l_{C}$ [determined by means of Eq. (31)] are plotted in Fig. 9(a) as a function of the applied magnetic field, which is usually the control parameter in magnetic SANS experiments. For the Nd-Fe-B nanocomposite (with $\Delta M \cong 0$ ), we expect that $l_{C}$ describes the

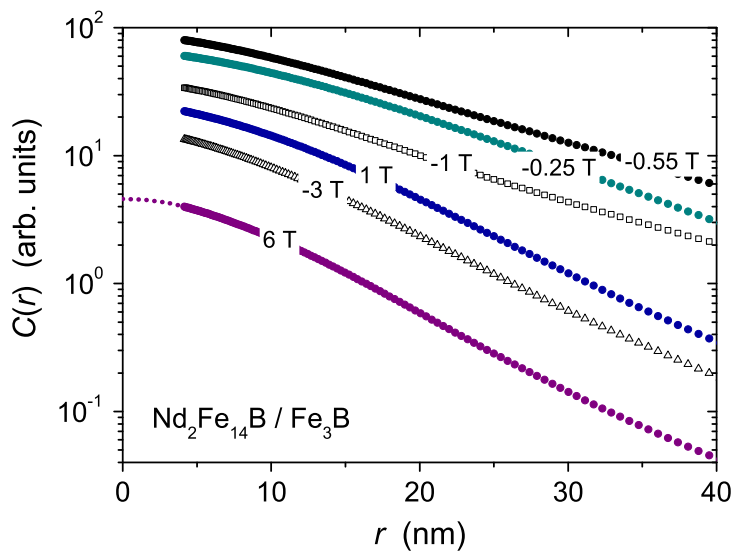

FIG. 8. Field dependence of the correlation function $C(r)$ of the spin misalignment of nanocrystalline $\mathrm{Nd}_{2} \mathrm{Fe}_{14} \mathrm{~B} / \mathrm{Fe}_{3} \mathrm{~B}$ (log-linear scale). The field values follow the course of a hysteresis loop, starting from a large positive field and then reducing the field to negative values (see insets). Dotted line (extrapolating the $6 \mathrm{~T}$ data to $r=0$ ): $C(r)=4.58-0.043 r^{2}$. From Bick et al., 2013.
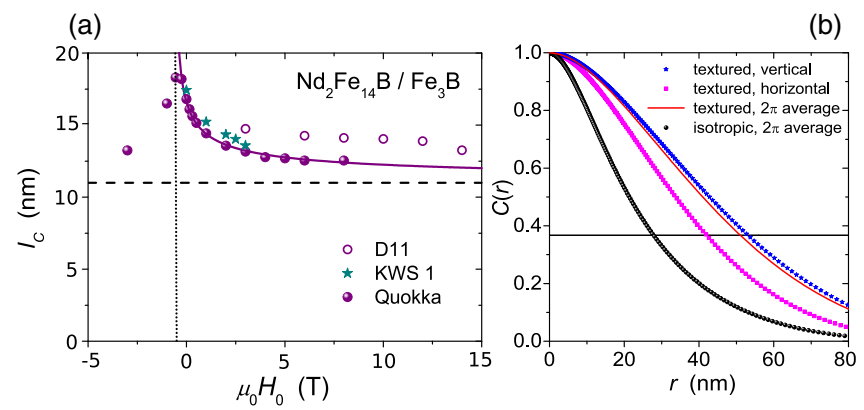

FIG. 9. (a) Applied-field dependence of the correlation length $l_{C}$ of the spin misalignment of nanocrystalline $\mathrm{Nd}_{2} \mathrm{Fe}_{14} \mathrm{~B} / \mathrm{Fe}_{3} \mathrm{~B}$. Solid line: fit of the data to Eq. (32), where $R=10.9 \mathrm{~nm}$ and $\mu_{0} H^{\star}=+0.60 \mathrm{~T}$ are treated as adjustable parameters, and the quantities $A=12.5 \mathrm{pJ} / \mathrm{m}$ and $\mu_{0} M_{s}=1.6 \mathrm{~T}$ are held fixed. In addition to $l_{C}\left(H_{0}\right)$ data obtained at the instrument Quokka (ANSTO), results obtained at the SANS instruments KWS 1 [Jülich Center for Neutron Science (JCNS)] and D11 [Institut Laue-Langevin (ILL)] are also shown. Dashed horizontal line: average radius of the $\mathrm{Nd}_{2} \mathrm{Fe}_{14} \mathrm{~B}$ particles $(R=11 \mathrm{~nm})$. Dotted vertical line: coercive field $\mu_{0} H_{c}=-0.55 \mathrm{~T}$. From Bick et al., 2013. (b) Normalized correlation function $C(r)$ of the spin misalignment for a textured (hot-deformed) and isotropic $\mathrm{Nd}_{2} \mathrm{Fe}_{14} \mathrm{~B} / \alpha$-Fe nanocomposite in the remanent state. The $C(r)$ of the textured sample is computed by using $d \Sigma_{M} / d \Omega$ averaged along the vertical and horizontal directions $\left( \pm 7.5^{\circ}\right.$ sector averages) as well as using the full circular $(2 \pi)$ average of $d \Sigma_{M} / d \Omega$; the $C(r)$ of the isotropic sample is computed using the corresponding $2 \pi$-averaged $d \Sigma_{M} / d \Omega$ (see inset). Solid horizontal line: $C(r)=e^{-1}$. Data from Michels et al. (2017).

spatial extent of magnetization inhomogeneities, mainly within the soft magnetic $\mathrm{Fe}_{3} \mathrm{~B}$ grains, that are caused by the jump in the magnetic materials parameters (exchange constant, direction, and magnitude of magnetic anisotropy) at the interface between the $\mathrm{Nd}_{2} \mathrm{Fe}_{14} \mathrm{~B}$ particles and the surrounding $\mathrm{Fe}_{3} \mathrm{~B}$ crystallites. As can be seen in Fig. 9(a), $l_{C}$ approaches a constant value of about $12.5 \mathrm{~nm}$ at the largest positive fields and increases with decreasing applied field to take on a maximum value of about $18.5 \mathrm{~nm}$ at the experimental coercive field of $\mu_{0} H_{c}=-0.55 \mathrm{~T}$. Further increase of $H_{0}$ toward more negative values results again in a decrease of $l_{C}$ toward $\sim 12.5 \mathrm{~nm}$. From the fit of the $l_{C}\left(H_{0}\right)$ data to Eq. (32) [solid line in Fig. 9(a)], we obtain $R=10.9 \mathrm{~nm}$ (close to the experimental average grain radius of the $\mathrm{Nd}_{2} \mathrm{Fe}_{14} \mathrm{~B}$ phase) and $\mu_{0} H^{\star}=+0.60 \mathrm{~T}$, which is close to the absolute value of the experimental coercive field. At the remanent state, the penetration depth of the spin disorder into the $\mathrm{Fe}_{3} \mathrm{~B}$ phase amounts to $\sim 5-6 \mathrm{~nm}$.

In a recent comparative study of the magnetic microstructure of textured and isotropic $\mathrm{Nd}_{2} \mathrm{Fe}_{14} \mathrm{~B} / \alpha-\mathrm{Fe}$ nanocomposites, evidence for a correlated crystallographic and spin texture was found (Michels et al., 2017). Specifically, the analysis of the neutron data suggested that the spinmisalignment scattering of the textured sample, prepared via melt spinning and subsequent hot deformation, is dominated by spin components along one direction perpendicular to the easy $c$ axis (pressing direction) of the $\mathrm{Nd}_{2} \mathrm{Fe}_{14} \mathrm{~B}$ grains. This anisotropy in the magnetization distribution is accompanied by the presence of a crystallographic texture along these 
directions, as revealed by $\mathrm{x}$-ray diffraction synchrotron data. Figure 9(b) illustrates the anisotropy of the correlations by depicting the correlation function of the textured nanocomposite; the correlation lengths along the horizontal $\left(l_{C} \cong 42 \mathrm{~nm}\right)$ and vertical $\left(l_{C} \cong 53 \mathrm{~nm}\right)$ directions differ considerably, which indicates differences in the magnetic interactions along these directions. This conjecture is supported by electron-microscopy and three-dimensional atomprobe tomography work (Liu et al., 2014), which reports anisotropic properties of the grain-boundary phase in hotdeformed nanocrystalline $\mathrm{Nd}-\mathrm{Fe}-\mathrm{B}$ magnets.

Sintered Nd-Fe-B-based permanent magnets, prepared via the powder metallurgical route, are presently more important from the economic perspective. Here the research focus is to reduce the amount of heavy rare-earth metals ( $\mathrm{Tb}$ and $\mathrm{Dy}$ ), which are added in order to guarantee the temperature stability of the magnet $\left(H_{c}\right)$ at the operating temperature of the device (e.g., $\sim 150{ }^{\circ} \mathrm{C}$ for electromotor applications). In this context, the effect of the grain-boundary diffusion process (GBDP) on the bulk magnetic microstructure of Tb-doped Nd-Fe-B-based sintered magnets was studied by Périgo et al. (2016) by means of magnetic-field-dependent unpolarized SANS. In the GBDP (Sepehri-Amin et al., 2010), the Nd-Fe-B magnet is exposed at elevated temperatures to a fine powder or a vapor containing high-magnetic-anisotropy-inducing heavy-rare-earth elements such as $\mathrm{Tb}$ or Dy, which then diffuse (preferentially along liquid grain boundaries) into the bulk of the material, in this way locally increasing the coercivity. Compared to the Tb-free sample, Périgo et al. (2016) observed in the GBDP specimen a $15 \%$ reduced correlation length and a $16 \%$ increased $H_{c}$ (see Fig. 10). The origin of the reduced correlation length is related to the increased local magnetic anisotropy field of the Tbenriched interfaces, which represent possible nucleation sites for reversed magnetic domains: the presence of $\mathrm{Tb}$ results in less magnetic disorder near or across the grain boundaries. Qualitatively, this finding agrees with the work of Saito et al. (2015) who reported reduced spatial fluctuations of magnetic

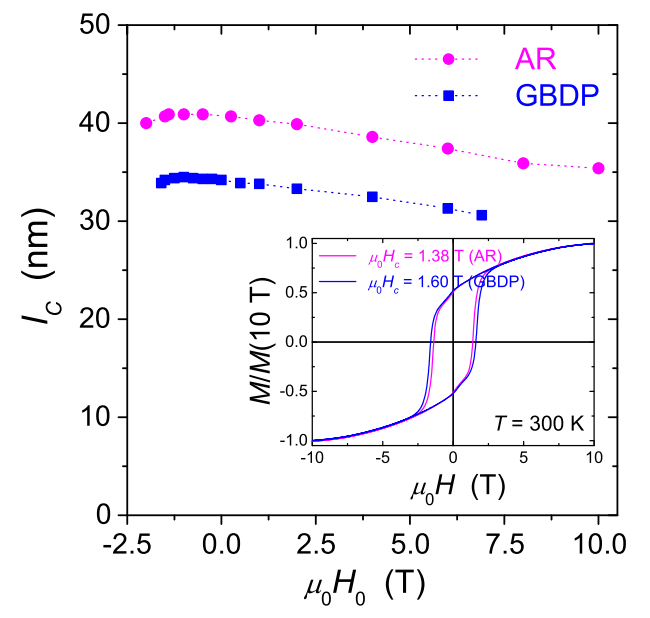

FIG. 10. The effect of the grain-boundary diffusion process (GBDP) on the field dependence of the correlation length $l_{C}$ of the spin misalignment of isotropic sintered Nd-Fe-B. The inset shows the hysteresis loops of the as-received (AR) and GBDP samples. Data from Périgo, Gilbert, and Michels (2015) and Périgo et al. (2016). moments due to the GBDP in $\mathrm{Nd}-\mathrm{Cu}$-infiltrated $\mathrm{Nd}-\mathrm{Fe}-\mathrm{B}$ nanocrystalline magnets.

SANS has also allowed the experimental observation of magnetic poles (due to $\nabla \cdot \mathbf{M} \neq 0$ ) existing in the bulk of magnetic materials. The signature of the magnetostatic interaction is a characteristic spike or flying-saucer-type pattern in $d \Sigma_{M} / d \Omega$ with sharp maxima for angles $\theta=0^{\circ}$ and $180^{\circ}$. Such an angular anisotropy was predicted by Weissmüller et al. (1999) and experimentally observed by Périgo et al. (2014) on an isotropic Nd-Fe-B-based permanent magnet (see Fig. 3). The spike anisotropy underlines the importance of the magnetodipolar interaction for understanding magnetic SANS.

Future neutron work will address the role of the intergranular Nd-rich layers for the coercivity mechanism of sintered magnets. Here the combination of micromagnetic simulations (see Sec. VI) with experimental SANS data might provide important insight into the signature of the grain boundaries in the magnetic SANS cross section.

\section{MAGNETIC STEELS}

This section aims to illustrate how magnetic SANS has contributed to the fast paced development of steels for applications under extreme conditions, such as those imposed by irradiation with fast neutrons $(E>1 \mathrm{MeV})$.

\section{A. New steels for application under extreme conditions}

The advancement, innovative application, and societal impact of steel have not ceased over the centuries. Currently, additional motivation for the development of new steels arises, for example, from extreme requirements, such as those imposed by nuclear fission (Zinkle and Was, 2013) and fusion (Knaster, Moeslang, and Muroga, 2016) environments. Recent progress in computational alloy design indeed indicates potential for the development of new steels.

The favorable property combinations of steels are partly achieved by manipulating the solid-state transformation from the high-temperature face-centered cubic (fcc) phase. Microstructures formed as a result include martensite and ferrite, which became eponyms for classes of steels. These steels have a body-centered cubic (bcc) lattice and exhibit ferromagnetism. It is also possible, for instance by alloying with $\mathrm{Ni}$, to stabilize the fcc phase to below room temperature. The resulting austenitic steels are paramagnetic and also experience wide application. It is, however, the ferromagnetism of the former steels that allows magnetic and nuclear scattering to be separated and additional information to be gained. By contrast, the ferromagnetism of a sample is challenging for transmission electron microscopy (TEM).

The application of SANS to ferromagnetic steels is partly at variance with other applications of magnetic SANS. Therefore, specific features of the approach will be introduced in Sec. IV.B.

The first field of application discussed here is related to low-alloy reactor pressure vessel (RPV) steels. Historically, mechanical testing of neutron-irradiated RPV steels indicated advancing embrittlement, obviously an issue of utmost significance for safe reactor operation. While the dominant embrittlement mechanisms were gradually understood 
(Odette and Lucas, 1998), special long-term irradiation effects are currently of interest (Altstadt et al., 2014). New insight into the nature and distribution of irradiation-induced nanofeatures derived from the application of magnetic SANS will be addressed in Sec. IV.C.

Components of future fission reactors and fusion devices will have to withstand more severe conditions, including neutron exposures up to 200 displacements per atom (dpa), a factor of 1000 more than RPV-typical exposures. 8\%-18\% Cr steels were found to be promising candidates. The contributions of magnetic SANS to an improved understanding of the $\mathrm{Fe}-\mathrm{Cr}$ system and the irradiation behavior of advanced $\mathrm{Cr}$ steels will be considered in Sec. IV.D.

Advanced oxide dispersion-strengthened (ODS) steels exhibit exceptional irradiation resistance due to a high density of internal point defect sinks and traps, e.g., grain boundaries and particle-matrix interfaces (Odette, Alinger, and Wirth, 2008). Information on the type and size distribution of oxide nanoparticles gained from the application of magnetic SANS will be highlighted in Sec. IV.E.

Before entering into a detailed discussion of the classes of steels previously introduced, other contributions of magnetic SANS to the development of new steels should be mentioned. These include ultra-high-strength steels combining the intermetallic strengthening mechanisms associated with maraging steels and alloy carbide strengthening (Eidenberger et al., 2011; Zhang et al., 2011; Delagnes et al., 2012; Perrut, Mathon, and Delagnes, 2012). The reported results indicate that the complex precipitation processes require the usage of combinations of complementary methods.

\section{B. Prerequisites}

We considered a two-phase material consisting of a dilute assembly of nanoparticles randomly dispersed in a ferromagnetic matrix. The basis for the separation of nuclear ("nuc") and magnetic ("mag") SANS is the application of a saturating magnetic field perpendicular to the unpolarized incident neutron beam [compare also Eq. (23) in Sec. II.D],

$$
\frac{d \Sigma}{d \Omega}(q, \theta)=\frac{d \Sigma_{\mathrm{nuc}}}{d \Omega}(q)+\frac{d \Sigma_{\mathrm{mag}}}{d \Omega}(q) \sin ^{2} \theta,
$$

where $\theta$ denotes the angle enclosed by the direction of the applied field and the momentum-transfer vector $\mathbf{q}$ [for conditions of validity of Eq. (36), see Bischof et al. (2007)]. The subsequent analysis procedure is similar for magnetic and nuclear SANS; the scattering cross sections of a suitable reference sample or a background is subtracted from the cross sections of the sample of interest. An inverse problem is solved for the difference scattering curve to obtain the size distribution of the additional scatterers. For the case of neutron-irradiated materials, the respective unirradiated sample is usually taken as reference. The outcome then is the distribution of irradiation-induced nanofeatures.

In order to calculate the size distribution in absolute units, the contrast, i.e., the square of the magnetic, Eq. (37), or nuclear, Eq. (38), difference scattering-length density between scatterer ("S") and matrix ("Ma") must be known:

$$
\begin{aligned}
\Delta \eta_{m} & =\left\langle\eta_{m}\right\rangle_{\mathrm{S}}-\left\langle\eta_{m}\right\rangle_{\mathrm{Ma}} \\
& =\sum_{i \in \mathrm{S}} n_{i, \mathrm{~S}} b_{m, i, \mathrm{~S}}-\sum_{i \in \mathrm{Ma}} n_{i, \mathrm{Ma}} b_{m, i, \mathrm{Ma}} \\
\Delta \eta_{n} & =\left\langle\eta_{n}\right\rangle_{\mathrm{S}}-\left\langle\eta_{n}\right\rangle_{\mathrm{Ma}} \\
& =\sum_{i \in \mathrm{S}} n_{i, \mathrm{~S}} b_{n, i}-\sum_{i \in \mathrm{Ma}} n_{i, \mathrm{Ma}} b_{n, i} .
\end{aligned}
$$

Here $n_{i}, \eta_{m, i}$, and $\eta_{n, i}$ are the atomic density, magnetic, and nuclear scattering-length densities of atomic species $i$, respectively. Contrary to the nuclear scattering length, the magnetic scattering length of an atom depends on the local environment. We provided the scattering lengths of $\mathrm{Cr}, \mathrm{Cu}, \mathrm{Fe}, \mathrm{Mn}$, and $\mathrm{Ni}$ in Table I for later reference.

The $A$ ratio was originally introduced as the ratio of the scattering cross sections perpendicular and parallel to the applied saturating magnetic field (Frisius and Buenemann, 1979):

$$
A=\frac{d \Sigma_{\perp} / d \Omega}{d \Sigma_{\|} / d \Omega}=1+\frac{d \Sigma_{\mathrm{mag}} / d \Omega}{d \Sigma_{\mathrm{nuc}} / d \Omega}=1+\frac{\left(\Delta \eta_{m}\right)^{2}}{\left(\Delta \eta_{n}\right)^{2}} .
$$

In general, $A$ is a function of $q$. As shown by Eq. (39), if the magnetic and nuclear scatterers are the same objects, $A$ can be expressed as the ratio of contrasts. Given the matrix structure and composition, Eqs. (37)-(39) describe a relationship between a measurable quantity of SANS and the structure and composition of the scatterers. The problem of extracting the composition of the scatterers from $A$ is underdetermined, except for the simplest systems and for nonmagnetic scatterers (magnetic holes). The contrast of magnetic holes is still used with some profit in the case of magnetic scatterers. Indeed, the volume fraction of scatterers obtained this way is a lower bound for all possible volume fractions, provided that $0<\left\langle b_{m}\right\rangle_{\mathrm{S}}<2\left\langle b_{m}\right\rangle_{\mathrm{Ma}}$.

A trial-and-error procedure to specify or narrow down the composition in the case of nonmagnetic scatterers is depicted in Fig. 11. As indicated therein by points $1-5$, the procedure consists of the following steps: 1 Calculate the experimental $A$ ratio from the separated magnetic and nuclear scattering. 2 Calculate the size distribution of precipitates from magnetic and nuclear scattering and check consistency between both. 3 Calculate the total volume fraction $f$ of scatterers in absolute units from magnetic scattering (known contrast) and in relative units from nuclear scattering (unknown contrast). 4 Assume a trial composition of the precipitates, calculate $A_{\mathrm{th}}$,

TABLE I. Magnetic and nuclear scattering lengths and resulting contrast of coherent precipitates in bcc Fe (contrast in multiples of the atom density $n_{\mathrm{Fe}}$ of bcc Fe, coherency strains ignored). $b_{m}$ values in parentheses correspond to substitutional impurities in Fe (Drittler et al., 1989). Magnetic contrasts in parentheses are for nonmagnetic precipitates.

\begin{tabular}{lcccc}
\hline \hline$i$ & $b_{m}(\mathrm{fm})$ & $\left(\Delta \eta_{m} / n_{\mathrm{Fe}}\right)^{2}$ & $b_{n}(\mathrm{fm})$ & $\left(\Delta \eta_{n} / n_{\mathrm{Fe}}\right)^{2}$ \\
\hline $\mathrm{Cr}$ & $(-3.5)$ & $(36)$ & 3.635 & 34 \\
$\mathrm{Cu}$ & 0 & 36 & 7.718 & 3.0 \\
$\mathrm{Fe}$ & 6 & 0 & 9.450 & 0 \\
$\mathrm{Mn}$ & $(1.9)$ & $(36)$ & -3.73 & 173 \\
$\mathrm{Ni}$ & 1.6 & 19.4 & 10.3 & 0.72 \\
\hline \hline
\end{tabular}




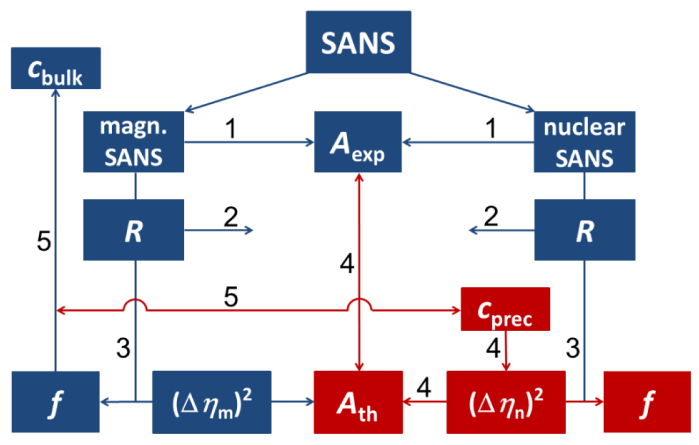

FIG. 11. Trial-and-error procedure to specify the composition $c_{\text {prec }}$ of precipitates for the case of nonmagnetic precipitates in ferromagnetic steels. The blue color indicates known or directly derived quantities. The red color indicates trials to be checked by way of comparison of both theoretical with experimental $A$ ratio and calculated with experimental bulk composition. The numbers refer to the sequence of steps (see the main text).

compare with $A_{\text {exp }}(\rightarrow$ error). 5 Use the same trial composition of precipitates along with $f$ to calculate the needed bulk concentrations. Compare with the bulk composition $(\rightarrow$ error). Take into account solubility limits if applicable. 6 Update the trials and iterate to reach self-consistency.

Alternatively, if the assumption of a dilute assembly of scatterers is violated, the Porod invariant can be used to calculate the volume fraction $f$ of scatterers (Porod, 1982b):

$$
\int_{0}^{\infty} \frac{d \Sigma_{\text {mag,nuc }}}{d \Omega} q^{2} d q=2 \pi^{2} f(1-f)\left(\Delta \eta_{m, n}\right)^{2} .
$$

Equation (40) is applicable independent of the structure of the two-phase medium and the degree of dilution of the system. Care has to be exercised for the proper extrapolation of the integrand.

Even at magnetic saturation, magnetic effects such as spin misalignment may give rise to significant deviations from Eq. (36) (Weissmüller et al., 2001) questioning the validity of the separation into magnetic and nuclear contributions. Bischof et al. (2007) derived corrections and analyzed a set of heat treatments of a martensitic steel to conclude that spinmisalignment corrections are particularly important for nuclear scattering in nanostructured steels. This correction requires magnetic-field-dependent SANS measurements.

Finally, it is important to note that spin-polarized neutrons allow the sign of the product $\Delta \eta_{m} \Delta \eta_{n}$ to be determined. This is impossible using conventional SANS and, therefore, may help to identify the type of scatterers in some cases, as underpinned in Secs. IV.C and IV.D.

\section{C. $\mathrm{Fe}-\mathrm{Cu}$ alloys and reactor pressure vessel steels}

Once the outstanding role of low levels of impurity $\mathrm{Cu}$ on the irradiation embrittlement of RPV steels was empirically recognized (Steele, 1975), both the investigation of simple model systems, such as binary $\mathrm{Fe}-\mathrm{Cu}$ alloys, and the application of new techniques with nm-scale sensitivity, such as SANS, entered the scene. SANS studies of the same model systems contributed to a deeper understanding of phase separation processes (Kampmann and Wagner, 1986) and provided input for the emerging multiscale modeling approach to irradiation damage. The utilization of $\mathrm{Cu}$ precipitation to replace carbide hardening of steels (Fine, Liu, and Asta, 2007) and the self-healing effect of $\mathrm{Cu}$ precipitation on steels (He et al., 2010) raised renewed interest.

Kampmann and Wagner (1986) studied $\mathrm{Cu}$ precipitation in thermally aged $\mathrm{Fe}_{1-x} \mathrm{Cu}_{x}(x=0.0138$ and 0.0064). The observed $\mathrm{Cu}$ precipitates were concluded to be nonmagnetic, which allowed the precipitate number density to be estimated in absolute units and models of the decomposition kinetics to be critically evaluated. He et al. (2010) performed timeresolved SANS on $\mathrm{Fe}_{1-x} \mathrm{Cu}_{x}(x=0.0098)$ during isothermal in situ aging at $550{ }^{\circ} \mathrm{C}$ in order to study $\mathrm{Cu}$-segregationassisted self-healing of deformation-induced defects. Assuming nonmagnetic precipitates at the measurement temperature, they derived the volume fractions of coexisting bcc, $9 R$, and $\mathrm{fcc} \mathrm{Cu}$ precipitates as a function of aging time. Schober et al. (2010) combined magnetic SANS and atomprobe tomography (APT) for thermally aged $\mathrm{Fe}_{1-x} \mathrm{Cu}_{x}$ $(x=0.0099)$ in order to address the issue of the $\mathrm{Fe}$ fraction in $\mathrm{Cu}$ precipitates for which levels between $0 \%$ and $50 \%$ had been reported before.

Several SANS studies of neutron-irradiated $\mathrm{Fe}-\mathrm{Cu}$ alloys were reported. Most notably, Miller, Wirth, and Odette (2003) recognized that nuclear SANS fed with the contrast derived from APT allows the Fe content of $\mathrm{Cu}$ precipitates to be crosschecked without assumptions on the precipitate magnetism. They found that, for irradiated $\mathrm{Fe}_{1-x} \mathrm{Cu}_{x}(x=0.008)$, the alloy $\mathrm{Cu}$ content needed to reconcile SANS with APT significantly exceeds the actual $\mathrm{Cu}$ content. Hence APT must have overestimated the $\mathrm{Fe}$ fraction in the $\mathrm{Cu}$ precipitates. Recently Shu et al. (2018), essentially based on temperaturedependent magnetic SANS, finally confirmed the presence of nonmagnetic $\mathrm{Cu}$ precipitates containing little or no $\mathrm{Fe}$ in neutron-irradiated and thermally aged $\mathrm{Fe}_{1-x} \mathrm{Cu}_{x}(x=0.008)$.

Regarding RPV steels, early SANS studies revealed irradiation-induced scatterers with a mean radius of approximately $1 \mathrm{~nm}$ for $\mathrm{Cu}$ impurity levels $\gtrsim 0.1 \mathrm{wt} \%$. It is now well understood that (i) coherent bcc $\mathrm{Cu}$-rich precipitates (CRPs) are the dominant type of nanofeatures formed as a result of neutron irradiation of $\mathrm{Cu}$-bearing steels at reactor ambient temperatures, $270-300^{\circ} \mathrm{C}$. CRPs may contain $\mathrm{Mn}, \mathrm{Ni}, \mathrm{Si}, \mathrm{Fe}$, and vacancies. (ii) Coherent or semicoherent $\mathrm{Mn}-\mathrm{Ni}(-\mathrm{Si})$ precipitates (MNPs) or their nonequilibrium precursors are the dominant type of nanofeatures in low-Cu, Mn-Ni-alloyed RPV steels (Odette, 1994). (iii) So-called matrix damage mainly consisting of dislocation loops and sub-nm vacancy clusters evolves slowly in all kinds of RPV steels. The contrast of the loops is too weak, and the vacancy clusters are too small, to be efficiently detected by SANS.

Increasing neutron exposure (fluence) gives rise to an increase of the volume fraction of CRPs at almost constant size. The effect of neutron flux (fluence rate) on the formation of CRPs is elucidated in Fig. 12. SANS, even in combination with APT, is not capable of unambiguously clarifying the average composition of multiconstituent precipitates in RPV steels (Carter et al., 2001; Hyde et al., 2014). Approaches aimed to isolate individual effects are crucial. Recent insight 


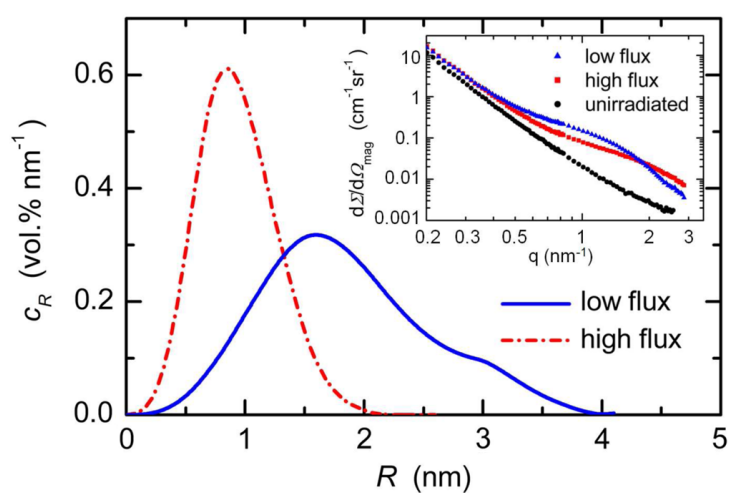

FIG. 12. Effect of neutron flux (for equal neutron exposure of $0.032 \mathrm{dpa}$ ) on the size distribution of irradiation-induced CRPs for an RPV weld $(0.22 \mathrm{wt} \% \mathrm{Cu}, 1.1 \mathrm{wt} \% \mathrm{Mn}, 1.1 \mathrm{wt} \% \mathrm{Ni})$. The measured magnetic scattering is shown in the inset. Low and high fluxes correspond to $0.087 \times 10^{-9}$ and $3.05 \times 10^{-9} \mathrm{dpa} / \mathrm{s}$ (11.6 years of irradiation), respectively. Data from Bergner et al. (2008), nonmagnetic scatterers assumed. The size of CRPs decreases, and the volume fraction is roughly constant.

into the roles of impurity $\mathrm{Cu}$ and alloying $\mathrm{Mn}$ and $\mathrm{Ni}$ derived from magnetic SANS is highlighted below.

Spin-polarized neutrons (Ulbricht, 2006) indicated $\Delta \eta_{m} \Delta \eta_{n}>0$ for the nanofeatures formed in a neutronirradiated RPV steel containing 0.2 wt $\% \mathrm{Cu}$ and 0.8 wt \% $\mathrm{Ni}$. This excludes the dominance of coherent $\mathrm{Ni}$ clusters $\left(b_{n, \mathrm{Ni}}>b_{n, \mathrm{Fe}}\right)$ and supports CRPs instead (see Table I).

For two neutron-irradiated $\mathrm{Fe}-\mathrm{Mn}-\mathrm{Ni}(-\mathrm{Cu})$ model alloys containing $1.2 \mathrm{wt} \% \mathrm{Mn}$ (both), $0.7 \mathrm{wt} \% \mathrm{Ni}$ (both) and $0.1 \mathrm{wt}$ $\% \mathrm{Cu}$ (second alloy only), $A<1.4$ (the value for voids) was observed for the lowest exposure of 0.051 dpa (Bergner et al., 2010). This indicates the dominance of $\mathrm{Mn}\left(b_{n, \mathrm{Mn}}<0\right.$, see Table I) in the early stage of solute cluster formation. These clusters are presumably related to the formation of "late blooming" MNPs at high fluences (Odette, 1994). Applying a combination of methods, Sprouster et al. (2016) confirmed that the MNPs identified after high-fluence irradiations are well-defined phases.

Böhmert, Viehrig, and Ulbricht (2004) and Wagner et al. (2012) reported pronounced linear correlations of the square root of precipitate volume fraction and irradiation hardening of RPV steels. The volume fractions were derived from magnetic SANS assuming nonmagnetic scatterers for the sets of RPV steels. This type of correlation is rationalized in the framework of the dispersed-barrier hardening model, which considers the obstruction of dislocation glide by randomly dispersed irradiation-induced obstacles, e.g., CRPs or MNPs.

\section{D. $\mathrm{Fe}-\mathrm{Cr}$ alloys and ferritic-martensitic $\mathrm{Cr}$ steels}

As mentioned in Sec. IV.A, 8\%-18\% Cr steels are promising candidates for nuclear applications. The hightemperature $\gamma$-phase field in the $\mathrm{Fe}-\mathrm{Cr}$ system extends to 11.9 wt $\% \mathrm{Cr}$. The martensitic transformation is only available below this level. The microstructure of $\mathrm{Cr}$ steels may be martensitic or ferritic depending on the cooling rate (transformable or ferritic-martensitic steels). At higher $\mathrm{Cr}$ contents, the steels are always ferritic (ferritic stainless steels).
$\mathrm{Cr}$ contents in excess of the solubility limit of $\mathrm{Cr}$ in $\mathrm{Fe}$ below $500{ }^{\circ} \mathrm{C}$ give rise to phase separation into Fe-rich $\alpha$ and $\mathrm{Cr}$-rich $\alpha^{\prime}$. The formation of $\alpha^{\prime}$ is responsible for the so-called $475^{\circ} \mathrm{C}$ embrittlement during thermal aging and for the embrittlement during neutron irradiation of $\gtrsim 9 \% \mathrm{Cr}$ steels. $\alpha$ and $\alpha^{\prime}$ are both bcc with only minor differences of the lattice parameters. The wide miscibility gap and small misfit strains make the $\mathrm{Fe}-\mathrm{Cr}$ system particularly attractive for the investigation of phase separation by spinodal decomposition. SANS is well suited because both magnetic and nuclear scattering lengths sufficiently differ from $\mathrm{Cr}$ to Fe. Moreover, magnetic and nuclear contrasts are of similar magnitude, contrary to Fe-Cu with $\Delta \eta_{m} \gg \Delta \eta_{n}$ (see Table I).

SANS was applied to investigate spinodal decomposition in $\mathrm{Fe}_{1-x} \mathrm{Cr}_{x}$ (typically $x>0.25$ ) (Vintaykin and Kolontsov, 1968; Katano and Iizumi, 1984; Bley, 1992; Ujihara and Osamura, 2000; Xu et al., 2016). These studies have in common that no external magnetic field was applied to the samples; for a comment see LaSalle and Schwartz (1986).

The determination of the solubility limit of $\mathrm{Cr}$ in $\alpha$ below $500{ }^{\circ} \mathrm{C}$ ( $\mathrm{Fe}$ solvus line of the $\mathrm{Fe}-\mathrm{Cr}$ phase diagram) is challenging because $\alpha-\alpha^{\prime}$ phase separation via nucleation and growth is extremely slow. Bonny, Terentyev, and Malerba (2008) concluded from reviewed experimental data that the solvus line according to the standard phase diagram must be shifted to higher $\mathrm{Cr}$ concentrations. Bergner, Ulbricht, and Heintze (2009), using Eq. (40), derived the volume fraction of $\alpha^{\prime}$ from SANS experiments performed on industrial-purity $\mathrm{Fe}_{1-x} \mathrm{Cr}_{x}(x=0.125)$ neutron irradiated at $300^{\circ} \mathrm{C}$ and found that a quasisteady state of $\mathrm{Cr}$ precipitation had been reached. The measured $A$ ratio $A=2.05 \pm 0.1$ was independent of $q$ and agreed with the value for $\alpha^{\prime}$ particles, $A=2.1$ (Mathon et al., 2003) (see Fig. 13). In view of the technical importance, a strict estimation of the solvus line requires additional attention.

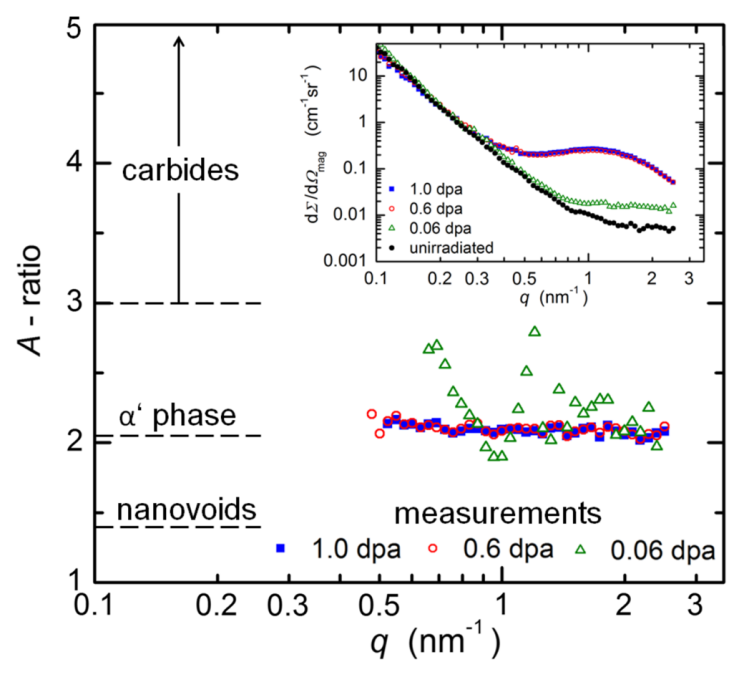

FIG. 13. The effect of neutron exposure on $A(q)$ for neutronirradiated $\mathrm{Fe}_{1-x} \mathrm{Cr}_{x}(x=0.125)$. The measured magnetic scattering is shown in the inset. The unirradiated reference was subtracted before calculating $A(q)$. Theoretical $A$ ratios are indicated. 
$\mathrm{Fe}-\mathrm{Cr}$ alloys also attracted interest as model systems aiming to explore the irradiation behavior of $\mathrm{Cr}$ steels. A set of neutron-irradiated $\mathrm{Fe}_{1-x} \mathrm{Cr}_{x}$ alloys $(x=0.025-0.125)$ was characterized by means of magnetic SANS and APT. The fraction of $41.5 \% \mathrm{Fe}$ in $\alpha^{\prime}$ found by APT was inconsistent with nuclear SANS (Bergner et al., 2013). Further clarification is required.

Briggs et al. (2017) reported results of a combined APT and SANS investigation of $\alpha^{\prime}$ precipitation in neutron-irradiated $\mathrm{Fe}-\mathrm{Cr}-\mathrm{Al}$ alloys to conclude about the role of $\mathrm{Al}$. As pointed out by Briggs et al., no magnetic field was applied and the measured total scattering was assumed to represent nuclear scattering, which gives rise to an overestimation of the volume fraction of $\alpha^{\prime}$. In retrospect, it seems worthwhile to quantify the degree of overestimation. Indeed, the isotropic magnetic scattering in the demagnetized state of the sample attains $2 / 3$ of the magnetic scattering contrast (Hyde et al., 2014).

Regarding Cr steels, Coppola et al. (1998) used spinpolarized neutrons to investigate an unirradiated $10.5 \mathrm{wt} \% \mathrm{Cr}$ steel austenitized at $1075^{\circ} \mathrm{C}$ and quenched in air. Negative values of the product $\Delta \eta_{m} \Delta \eta_{n}$ were found for the size range 1-10 nm, which allowed the dominant type of scatterers to be identified as Fe-rich carbides as opposed to $\mathrm{Cr}$-rich carbides.

Mathon et al. (2003) applied magnetic SANS to investigate a number of neutron-irradiated $7 \%-12 \% \mathrm{Cr}$ steels. Calculation of the $A$ ratio required a number of uncertainties related to the composition and magnetic scattering-length densities of $\alpha$ and $\alpha^{\prime}$ be addressed. Mathon et al. (2003) proved that the calculated $A$ ratio depends only weakly on these factors. Comparison with the measured $A$ ratios allowed $\alpha^{\prime}$-phase particles to be identified as the dominant irradiation-induced nanofeatures for all but the lowest $\mathrm{Cr}$ contents, 7.5 and $8.4 \mathrm{wt}$ $\%$. Moreover, irradiation-accelerated rather than irradiationinduced precipitation of $\alpha^{\prime}$-phase particles was suggested. These results gave fresh impetus for the reconsideration of the $\mathrm{Fe}$ solvus in the binary $\mathrm{Fe}-\mathrm{Cr}$ equilibrium phase diagram (see earlier discussion).

\section{E. Advanced oxide dispersion-strengthened steels}

Advanced ODS Cr steels, also referred to as nanostructured transformable steels $(<12 \% \mathrm{Cr})$ or nanostructured ferritic alloys $(>12 \% \mathrm{Cr})$, are distinguished by their radiation resistance (Ukai and Fujiwara, 2002; Odette, Alinger, and Wirth, 2008). The standard fabrication route is based on powder metallurgy including the steps of mechanical alloying by means of high-energy ball milling of a prealloy powder blended with typically $0.2-0.5 \mathrm{wt} \%$ yttria powder and consolidation, mainly by means of hot isostatic pressing (HIP).

As oxide nanoparticles are typically nonmagnetic, the volume fraction can be derived in absolute units from magnetic SANS. The $A$ ratio is particularly useful to check hypotheses on the type of oxides dominating in macroscopic samples (see Fig. 11). The selection of a suitable reference sample or baseline for SANS is an issue: Y-free counterparts produced in parallel with ODS steels (Alinger, Odette, and Hoelzer, 2004; Pareja et al., 2015) do not only differ from the ODS samples with respect to the absence of Y-bearing oxides (intended difference), but also with respect to other features, such as the presence of $\mathrm{Cr}$ - and Ti-bearing oxides (side effect).

Magnetic SANS was applied to examine advanced ODS steels across all development stages and production steps. The effect of milling parameters on the size distribution and type of oxide nanoparticles was addressed by investigating as-milled powders (Alinger, Odette, and Hoelzer, 2004; Mathon et al., 2012) or consolidated samples with the consolidation parameters kept constant (Hilger et al., 2016). Alinger, Odette, and Hoelzer (2004) studied ODS alloys with the composition and processing parameters varied systematically and found that both $\mathrm{Y}$ (in the form of added yttria powder) and $\mathrm{Ti}$ (as a constituent of the prealloy) are required for a high number density of oxide nanoparticles to be formed during HIP. Mathon et al. (2012), supplemented by Hilger et al. (2016), tabulated $A$ ratios for different kinds of Y-, Cr-, Ticontaining equilibrium and nonequilibrium oxides in $\mathrm{Fe}-\mathrm{Cr}$ alloys. The comparison of measured (2.0-2.8) and calculated $A$ ratios proved useful to rule out a number of reported candidates for the dominant type of nanoparticles, such as $\mathrm{Y}_{2} \mathrm{O}_{3}(A=3.2)$. It is worth noting that the measured $A$ ratio is a good indicator of the particles' Y:Ti ratio because of the different nuclear scattering lengths of $\mathrm{Y}\left(b_{n}=7.75 \mathrm{fm}\right)$ and $\mathrm{Ti}\left(b_{n}=-3.37 \mathrm{fm}\right)$. However, the variation of the dominant types of nanoparticles as a function of the processing parameters is still not exhaustively understood.

Wang et al. (2012) performed in situ annealing at continuously increasing temperatures up to $1400^{\circ} \mathrm{C}$ in SANS experiments on a $14 \% \mathrm{Cr}$ nanostructured ferritic alloy. No magnetic field was applied, raising questions on the role of magnetic scattering. Nonetheless, the nanoclusters were found to persist up to $1400^{\circ} \mathrm{C}$ indicating unusual thermal stability. The robust measures of particle radius and volume fraction derived from SANS give rise to pronounced correlations with hardness and yield stress (Alinger, Odette, and Hoelzer, 2004; Zhong et al., 2016).

\section{F. Summary on magnetic steels}

Magnetic SANS has contributed significantly to the understanding of the behavior of ferromagnetic RPV steels, high-Cr steels, and advanced ODS steels in extreme environments. It is the ferromagnetism that allows magnetic and nuclear scattering to be separated, the number density of scatterers to be estimated, and the $A$ ratio as an indicator of composition to be exploited. The possibility of Fe fractions in nm-sized precipitates substantially in excess of equilibrium levels would impede the derivation of volume fractions and number densities from SANS results. However, such excess levels seem to be generally unfounded. By contrast, it is suggested to use SANS in combination with APT in order to calibrate the $\mathrm{Fe}$ fraction in cases of doubt. A number of material-specific open issues have already been identified. As material trends move toward nanostructured steels, strict interpretation of SANS results increasingly requires spin-misalignment scattering to be addressed. Samples of complex shape or in situ treatments may prevent magnetic saturation from being reached in SANS experiments. Refined strategies to estimate volume fractions and number densities under such circumstances are desirable. 


\section{MAGNETIC NANOPARTICLES AND FERROFLUIDS}

In this section we review the use of SANS for the investigation of magnetic nanoparticles and ferrofluids. Magnetic nanoparticles mostly consist of an inorganic magnetic nanoparticle core surrounded by a nonmagnetic stabilizing shell of either organic ligands, polymers, or inorganic materials. Below the critical size limit, the nanoparticle demagnetization energy is not sufficient to compensate the domain-wall energy, resulting in a single-domain state. Single-domain nanoparticles have different magnetization relaxation characteristics than the bulk material, leading to phenomena such as superparamagnetism, covered in detail by Bedanta and Kleemann (2009). The distinct magnetization relaxation behavior of nanoparticles gives rise to diverse applications, e.g., in information technologies or medical applications such as medical imaging and magnetic hyperthermia. Colloidal dispersions of single-domain magnetic nanoparticles in a carrier liquid (Pankhurst et al., 2003; Bader, 2006) are referred to as ferrofluids and find application in high-vacuum gears, seals, loudspeakers, sensors, etc. (Joseph and Mathew, 2014). Important parameters regarding the preparation and application of ferrofluids include the magnetic material of the nanoparticles and the stability against precipitation.

The synthesis of magnetic nanoparticles can be carried out by either top-down techniques (starting with the bulk material) or bottom-up approaches (building up the material from atomic or molecular species) (Lu, Salabas, and Schüth, 2007; Park et al., 2007; Xia et al., 2009; Joseph and Mathew, 2014), with the latter generally preferred for enhanced sample homogeneity on the nanoscale. Co-precipitation has been widely applied as a large-scale bottom-up technique, at the expense of moderate particle-size distribution. Large-scale synthesis of monodisperse nanoparticles (e.g., ferrites) with defined shape is nowadays routinely achieved by thermal decomposition of metal oleates in the presence of organic stabilizing ligands (Park et al., 2004, 2005). Stabilization of the prepared nanoparticles in dispersion is important in order to avoid aggregation and precipitation and depends strongly on the nature of the carrier liquid (polar or nonpolar) and the stabilizing approach (steric stabilization using surfactants, polymers, or electrostatic stabilization) (Joseph and Mathew, 2014).

Major challenges addressed by SANS include the intraparticle magnetization, i.e., the spatial magnetization distribution within magnetic nanoparticles, and interparticle structure formation, i.e., aggregate or superstructure formation induced by dipolar interparticle interactions. Moreover, SANS gives insight into microstructural aspects of magnetohydrodynamics and magnetoviscosity of ferrofluids.

We start the discussion in Sec. V.A by introducing the relevant cross-section relations in the framework of the particle-matrix approach. Section V.B reviews the contribution of magnetic SANS to the investigation of the intraparticle magnetic morphology, including the spatially resolved magnetization profile in nearly uniformly magnetized nanoparticles, whereas Sec. V.C focuses on the application of magnetic SANS to the structure formation in more concentrated ferrofluids. In Sec. V.D we summarize recent developments concerning the magnetic structure of shapeanisotropic nanoparticles, such as oriented nanowires in porous alumina matrices.

\section{A. Particle-matrix approach}

As already stated in Secs. I and II, magnetic SANS is sensitive to a length scale relevant for magnetic nanoparticles in a nonmagnetic matrix. The particle-matrix approach, consisting of nuclear and magnetic form-factor contributions of the individual nanoparticles as well as a structure factor describing potential interparticle interactions, is therefore widely used to describe SANS data from these systems.

To apply a form-factor model $F(\mathbf{q},\{R, \ldots\})$, the different averaging procedures to obtain a macroscopic cross section from the microscopic cross sections for individual particles are of particular importance. This intrinsic property of SANS is connected to the limited size of the coherence volume of the neutron in the beam (Felber et al., 1998). A system of scatters in the particle-matrix approach is called diluted, if the neutron coherence volume contains on the average only a single particle. If, on the other hand, one aims for the investigation of structure formation that volume has to be large enough so that the neutron can probe particle-particle interferences (see Sec. V.C). To a great extent, and with some exceptions [see, e.g., Grigoriev et al. (2010)], this coherence volume is defined by the instrument setup. Assuming a diluted system of nanoparticles in a neutron beam with an average typical flux of the modern instruments of the order of $10^{7} \mathrm{~s}^{-1} \mathrm{~cm}^{-2}$, different neutrons will scatter on different particles in a nonconstructive way. At the 2D detector (Fig. 1), the sum of the squared amplitudes is then related to the count rate and normalized by the scattering volume and incident flux to obtain the macroscopic SANS cross section.

In most experimental situations, the individual particles have slightly different properties, e.g., different radii, and a global average over a particle-size distribution function $\omega(R)$ has to be part of the data-fitting procedure; in other words, the sum of the squared amplitudes is often substituted by an integral assuming $\omega(R)$ to be a continuous function, and the nuclear SANS cross section is expressed as

$$
\frac{d \Sigma}{d \Omega}=\int \Delta \eta_{n}^{2}\left|F_{n}(q, R)\right|^{2} \omega(R) d R,
$$

where $\Delta \eta_{n}$ denotes the nuclear scattering-length density (SLD) contrast between particle and matrix (generally assumed to be independent of $R$ ). For magnetic SANS, described by a magnetic form factor $F_{m}(q, R)$ and the magnetic SLD contrast $\Delta \eta_{m}$, a similar expression is valid. As shown in Sec. II.D, the magnetic SLD contrast $\Delta \eta_{m}=\eta_{m}$ between magnetic particle and nonmagnetic matrix can be written as $\eta_{m}=b_{H} M_{s}$, where $b_{H}=\left(\gamma_{n} r_{0} / 2\right) \mu_{B}^{-1}$ and $M_{s}$ is the saturation magnetization (in $\mathrm{A} / \mathrm{m}$ ) of the particle. For single-domain (Stoner-Wohlfarth) particles exhibiting stable ferromagnetism, Eq. (41) has to be solved directly by employing a particular form-factor model (or even a structure factor); in case of inhomogeneously magnetized nanoparticles the magnetization distribution has to be computed using 
micromagnetic theory and the SANS cross sections are given by Eqs. (12)-(20).

In the following we will discuss how the magnetic SANS cross section of superparamagnetic (SPM) or pseudo-SPM particles in a nonmagnetic matrix can be computed; here an additional averaging procedure over the orientation distribution $p(\varphi)$ of the individual magnetic particle moment misaligned by the angle $\varphi$ relative to the field direction becomes relevant. For a single-domain particle, one can assume an orientation distribution based on the thermal and magneticenergy competition. If $d \Omega=\sin \varphi d \varphi d \phi$ is the solid angle increment in a polar system where the $z$ direction is given by the external field $\mathbf{H}_{0}$, then the orientational average over $\varphi$ is described by the probability distribution

$$
p(\varphi) \propto \exp \frac{\mu_{0} H_{0} M_{s} V_{p} \cos \varphi}{k T}
$$

where $M_{s}$ is the saturation magnetization of the particle with volume $V_{p}$. In this model a single neutron interacts with the magnetization vector of the particle which is lying on a cone with solid angle $\omega=(\varphi, \phi)$ around the external-field axis. Because the $\phi$ component is independent of any external field, the distribution is random and will not introduce any anisotropy. This is different for the $\varphi$ component; it directly reflects the influence of the external magnetic field (and temperature) via Eq. (42), leading to a general scatteringintensity pattern of the type

$$
I(q)=A(q)+B(q) \sin ^{2} \theta .
$$

In case of moderately polydisperse systems, the double average $\iint \omega(R) p(\varphi) d R d \varphi$ can be divided into two separate parts. If $[\cdots]$ denotes the directional average $[x]=$ $\int x(\varphi) p(\varphi) d \varphi$, it allows the analytical calculation of all the contributions in Eqs. (12)-(20). The required magnetization averages are as follows:

$$
\begin{aligned}
{\left[\widetilde{M}_{x}\right] } & =\left[\widetilde{M}_{y}\right]=0 \quad \text { and }\left[\widetilde{M}_{z}\right]=F_{m} L(\beta), \\
{\left[\left|\widetilde{M}_{x}\right|^{2}\right] } & =\left[\left|\widetilde{M}_{y}\right|^{2}\right]=F_{m}^{2} \frac{L(\beta)}{\beta}, \\
{\left[\left|\widetilde{M}_{z}\right|^{2}\right] } & =F_{m}^{2}\left(1-2 \frac{L(\beta)}{\beta}\right),
\end{aligned}
$$

so that the SANSPOL cross section in the $\mathbf{k}_{0} \perp \mathbf{H}_{0}$ scattering geometry [Eq. (14)] evaluates to [for details see, e.g., Wiedenmann (2001, 2005), Heinemann and Wiedenmann (2003), and Heinemann, Wiedenmann, and Kammel (2004)]

$$
\begin{aligned}
\frac{d \Sigma_{\perp}^{ \pm}}{d \Omega}= & \int \omega(R) d R\left[F_{n}^{2}+2 F_{m}^{2} \frac{L(\beta)}{\beta}+\left\{F_{m}^{2}\left(1-3 \frac{L(\beta)}{\beta}\right)\right.\right. \\
& \left.\left.+(2 P-1)\left(2 \epsilon^{ \pm}-1\right) F_{n} F_{m} L(\beta)\right\} \sin ^{2} \theta\right],
\end{aligned}
$$

where $F_{m}$ and $F_{n}$ are the magnetic and nuclear form factors, $L(\beta)=\operatorname{coth}(\beta)-1 / \beta$ is the Langevin function with $\beta=\mu_{0} H_{0} M_{s} V_{p} / k T$, and $\theta$ is the angle between the magnetic field and the q vector. One can observe that $A(q)$ in Eq. (43) now contains not only the nuclear SANS but also magnetic contributions; these originate from the distribution of the magnetic moments around the external field and decrease with increasing field $[L(\beta) / \beta \rightarrow 0$ for $\beta \rightarrow \infty$ and $L(\beta) / \beta \rightarrow 1 / 3$ for $\beta \rightarrow 0]$. Magnetic field $\left(\mathbf{H}_{0}\right)$ or temperature $(T)$ variation change $\beta$ and thereby the fractions of $A(q)$ and $B(q)$ (Heinemann and Wiedenmann, 2003; Heinemann, Wiedenmann, and Kammel, 2004). For SANSPOL the $B(q)$ part now also contains nuclear-magnetic interference contributions. This is not the case for unpolarized SANS and can be used to improve the data analysis. If the nuclear $F_{n}(q, R)$ and magnetic $F_{m}(q, R)$ form factors depend on the same particle-size distribution, the particle-matrix approach from Eq. (45) leads to a simple equation for the intensity differences [flipper on $\left(\epsilon^{-}=\epsilon\right)$ minus flipper off $\left(\epsilon^{+}=0\right)$ ]:

$$
\begin{aligned}
\frac{d \Sigma_{\perp}^{-}}{d \Omega}-\frac{d \Sigma_{\perp}^{+}}{d \Omega}= & 2(2 P-1) \epsilon \int L\left(H_{0}, R^{3}\right) F_{n}(R) F_{m}(R) \\
& \times \sin ^{2} \theta \omega(R) d R .
\end{aligned}
$$

In this cross term only particles with nuclear and magnetic contrast will contribute; this offers the possibility to detect contributions of the magnetic scattering without the influence of properties from purely nonmagnetic scatterers such as, e.g., micelles. For SANSPOL experiments, the cross-term intensity Eq. (46) is proportional to $L(\beta)$ and to the magnetic contrast (included in $F_{m}$ ) which is assumed to be field independent for single-domain particles. This allows a straightforward testing of the Langevin behavior by magnetic field variation experiments.

Figure 14(a) displays field-dependent SANS data obtained on a Co-based ferrofluid. The cross term Eq. (46) was fitted under the constraint that all structural contributions such as form factors are field independent and the overall intensity scales with the magnetic contrast via the Langevin function only. Taking the Langevin behavior hypothesis as settled, one can apply a Bayesian method to refine any structural model by
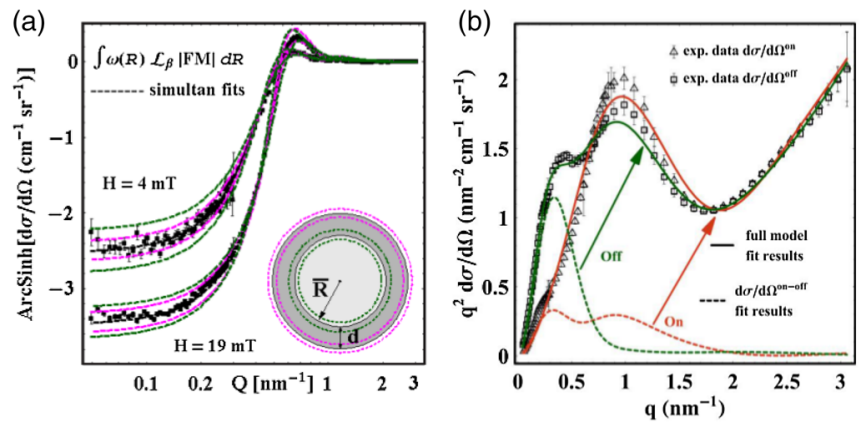

FIG. 14. (a) Experimental SANS data of a Co-based ferrofluid. The black lines are the results of a simultaneous fit of Eq. (46) for different external fields. The inset shows the core-shell particle with confidence intervals for the core radius (green dashed line) and shell thickness (pink dashed line). (b) Fits of the cross term lead to dashed curves within the full flipper on and off data. The missing intensity originates from nonmagnetic contributions and can be fitted separately. From Heinemann, Wiedenmann, and Kammel, 2004. 
magnetic contrast variation (Tatchev et al., 2004). An additional field will contribute to a better a posteriori information about the model and can be used to reduce the uncertainty levels for the model parameter. For clarity, Fig. 14(a) shows only two fields, but the model was fitted with four different fields (including the saturation field) for the final confidence intervals. In principle this is already possible with unpolarized neutrons but as one can see from Eq. (45), the nonsaturated parts give rise to an isotropic contribution leading to bigger error margins in the nuclear-magnetic scattering separation. In the case of a second population of nonmagnetic scatterers such as micelles, it will become very demanding to separate these contributions without the use of polarized neutrons. By analyzing the cross term to separate the structural properties from the magnetic contributions, it is possible to fit contributions from the additional nonmagnetic parts [see Fig. 14(b)].

With POLARIS (SANS with 1D polarization analysis) one can go one step further by analyzing the spin-flip only contributions from Eq. (18) in more detail. We showed that for pure superparamagnetic behavior the resulting SANS cross section can be written as a sum of two contributions, Eq. (43), where $A(q)=0$ for the $I^{-}-I^{+}$term. Therefore, it becomes possible to extract $A(q)$ and $B(q)$ because of their known angular dependence. Deviation from superparamagnetic behavior based on a more complex internal spin structure of the nanoparticles would lead to additional terms with different angular dependence due to the occurrence of coherent scattering arising from transversal magnetization components $M_{x}$ and $M_{y}$ [compare, e.g., Eq. (18)]. Although this could be observed already within the SANSPOL frame, it would be very hard to extract these additional contributions unambiguously. POLARIS, and, in particular, the spin-flip term, opens the possibility to observe these contributions. Using the same averaging procedure as in Eq. (44), one obtains for the macroscopic spin-flip cross section

$$
\begin{aligned}
\frac{d \Sigma_{\perp}^{ \pm \mp}}{d \Omega} \propto & \int d \varphi p(\varphi)\left(\left|\widetilde{M}_{x}\right|^{2}+\left|\widetilde{M}_{y}\right|^{2} \cos ^{4} \theta\right. \\
& \left.+\left|\widetilde{M}_{z}\right|^{2} \sin ^{2} \theta \cos ^{2} \theta-2 \widetilde{M}_{y} \widetilde{M}_{z} \sin \theta \cos ^{3} \theta\right) .
\end{aligned}
$$

The $\Sigma_{\perp}^{+-}$part is identical to the $\Sigma_{\perp}^{-+}$part and contains only magnetic contributions [see, e.g., Wiedenmann (2005) and Honecker et al. (2010)]. Depending on the model chosen for the distribution of magnetic moments, not necessarily following a Langevin behavior, one can try to identify newly occurring anisotropic scattering patterns [see, e.g., Krycka et al. (2010a, 2010b, 2012, 2014, 2015) and Michels et al. (2015)]. In all cases a model-based fitting of the full 2D detector pattern or full-fledged extraction of the different angle-dependent contributions, as in SANSPOL, is preferable.

Moreover, it must be emphasized that all additional corrections for the imperfect analyzers, flippers, and the different transmissions for up and down neutrons have to be taken into account carefully to minimize spin leakage (Wildes, 2006). Because the nuclear scattering can be dominating, even small leakages could lead to misinterpretation of the obtained data. Although in principle POLARIS allows one to unambiguously distinguish between magnetic and nuclear coherent scattering, these experimental complications make such experiments more demanding and error vulnerable.

\section{B. Intraparticle magnetization}

The spin structure of magnetic nanoparticles can be regarded as a superspin of coupled atomic spins within the particle core. Depending on particle volume, magnetic anisotropy, and thermal energy, Néel relaxation of the superspin is either blocked or dominant, giving rise to superparamagnetism. The existence of a magnetically dead or canted layer toward the particle surface has been widely reported, mainly based on theory and observations from macroscopic magnetization (Coey, 1971; Kodama et al., 1996; Kodama and Berkowitz, 1999). The lower saturation magnetization than observed in the bulk materials is generally attributed to such surface spin disorder (Curiale et al., 2009; Dutta et al., 2009; Kovács et al., 2009), leading to the generally accepted model of magnetic nanoparticles consisting of a superspin core and a surface region of canted or disordered spins. In contrast to spatially averaging and macroscopic integral probes, magnetic SANS has the unique strength to disentangle the spatial distribution of magnetization within the nanoparticle by analysis of the nuclear and magnetic particle sizes (indicating surface spin disorder) as well as the magnetic SLD $\eta_{m}$ (corresponding to the absolute magnetization).

In the first approach, the magnetic form factor can be regarded as similar or equal to the nuclear inorganic nanoparticle core. In this case, the magnetic contrast obtained by (polarized) SANS is beneficial for structural characterization of magnetic nanoparticles in a nonmagnetic matrix or stabilizing material such as an organic ligand shell (Hoell et al., 2002; Butter et al., 2004), an inorganic oxidized surface layer (Butter et al., 2004), or a silica shell (Bonini, Wiedenmann, and Baglioni, 2004, 2006, 2007). Avdeev and Aksenov (2010) established a modified basic functions approach that relies on a detailed contrast variation using $\mathrm{H}$ and $\mathrm{D}$ solvents. This approach allowed for a consistent determination of the nuclear particle morphology for dilute polydisperse nanoparticle dispersions stabilized by different fatty acids (Avdeev et al., 2007).

Heinemann and Wiedenmann further applied the additional contrast variation provided by polarized SANS to the structural characterization of magnetic nanoparticles with a nonmagnetic ligand shell next to excess surfactant (forming nonmagnetic micelles) and larger aggregates (composed of magnetic nanoparticles with nonmagnetic surfactant) (Heinemann, Wiedenmann, and Kammel, 2004; Heinemann et al., 2004; Wiedenmann, 2005; Wiedenmann, Kammel et al., 2006). By analyzing the radius of gyration of polydisperse maghemite nanoparticles, Avdeev et al. (2009) reported a difference between the nuclear and magnetic radii of maghemite nanoparticles in dilute dispersion which is attributed to surface spin canting or disorder effects. Significant variations between nuclear and magnetic structures are attributed to aggregates. The nuclear signal appears affected by van der Waals interactions at small $q$, whereas there is negligible dipolar magnetic interaction in the superparamagnetic particle ensemble. In order to exclude the influence of interparticle 
correlation, a decrease of the particle-number density in the magnetic fluids is suggested (Nagornyi et al., 2010).

With the availability of nearly monodisperse nanoparticles in large quantities, the discrimination of surface spin disorder has been enabled with enhanced precision. Using the POLARIS technique, Krycka et al. (2010a) studied the 3D magnetization distribution in dense arrays of $9 \mathrm{~nm}$ iron oxide nanoparticles. Assuming a field and temperature-independent structure factor, the significant difference of form factors in $M_{\|}$and $M_{\perp}$ indicates surface spin canting in a 1-1.5 nm thick surface region. Further analysis of the vectorial shell magnetization in $M_{\perp} / M_{\|}$using a core-shell form-factor model suggests an average canting angle of $23^{\circ}-31^{\circ}$ (at $1.2 \mathrm{~T}$ and $300 \mathrm{~K}$ ) (Krycka et al., 2014). The significant thickness of the canted surface layer found in these studies is likely a result of dipolar interactions in the dense nanoparticle arrays. The spatial magnetization distribution in noninteracting nanoparticles was investigated by Disch et al. (2012) in dilute dispersions of highly monodisperse maghemite nanoparticles using SANSPOL; surface spin disorder was revealed in a narrow surface layer that depends on the particle shape and is slightly thicker for nanocubes $(0.5 \mathrm{~nm})$ than for nanospheres $(0.3 \mathrm{~nm})$. More recently, Zákutná et al. reported surface spin disorder in noninteracting ferrite nanoparticles with a surface layer thickness of $0.3 \mathrm{~nm}$ in saturation, increasing up to $0.7 \mathrm{~nm}$ with decreasing applied magnetic field (Zákutná, 2019).

In addition to surface spin disorder, polarized SANS revealed an unexpectedly low magnetic scattering contrast in the particle core (Butter et al., 2004; Avdeev et al., 2009; Disch et al., 2012). It is noteworthy that the determination of the quantitative $\eta_{m}$ does not rely on calibration of the differential cross section to absolute units and thus cannot be mistaken for low particle-number densities. Krycka et al. (2010b) derived $\eta_{m}$ relative to the known nuclear SLD $\eta_{n}$ from the ratio $F_{m}^{2} / F_{n}^{2}$, assuming a phenomenological structure factor in assemblies of iron oxide nanoparticles. The magnetic SLD $\eta_{m}$ is a quantitative measure of the magnetization. As an example, Fig. 15 displays the field dependence of the core $\eta_{m}$ in maghemite nanocubes and nanospheres, revealing a spontaneous magnetization of only $76 \%$ of the bulk material, independent of the particle shape (Disch et al., 2012). The low magnetization even in the particle core thus indicates spin disorder in the entire nanoparticle, suggesting that the commonly observed lower macroscopic magnetization in nanoparticles as compared to the bulk material is not solely related to surface spin disorder, but to a large extent a result of low magnetization in the entire nanoparticle.

Given that SANS is sensitive to the continuous magnetization distribution on the $\mathrm{nm}$ scale, further characterization of the atomic-scale magnetization or spin disorder in magnetic nanoparticles requires the combination with theory or different techniques. The combination of POLARIS with an energybalance model was reported for the discussion of the average spin-canting angle, thereby assuming, among others, a perfect magnetite $\mathrm{Fe}_{3} \mathrm{O}_{4}$ composition and spin canting solely on the tetrahedral $\mathrm{Fe}$ sites of the spinel structure (Krycka et al., 2014). The study is under controversial debate for the different preconditions assumed (Krycka et al., 2015; Michels et al., 2015). Combining SANSPOL with nuclear-resonant $x$-ray

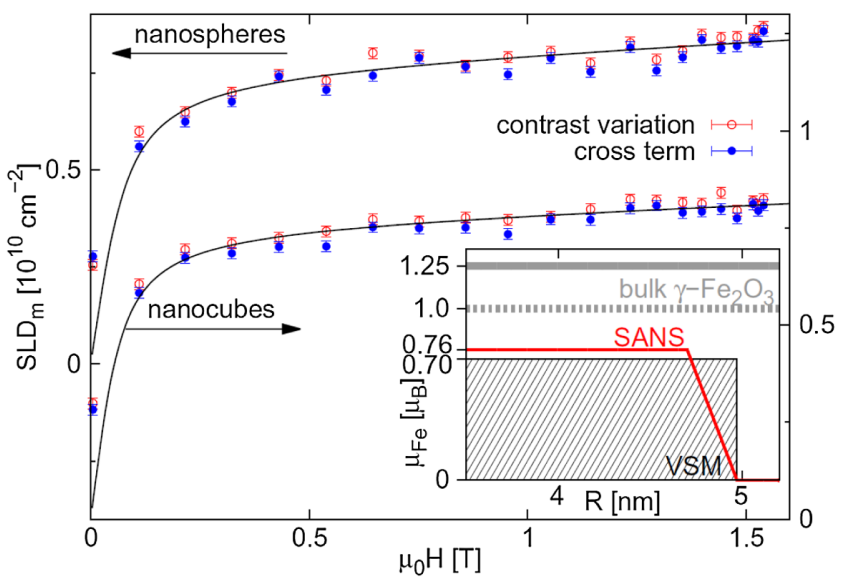

FIG. 15. Field dependence of the magnetic SLD $\eta_{m}$ in the nanoparticle core of maghemite nanospheres (top) and nanocubes (bottom) with fits of the Langevin behavior. Inset: spatial magnetization distribution of the nanospheres (SANS) compared to the macroscopic [vibrating sample magnetometry (VSM)] and the theoretical bulk maghemite moments (dashed line: $300 \mathrm{~K}$ ). From Disch et al., 2012.

scattering, Herlitschke et al. (2016) demonstrated a significant degree of spin disorder in ${ }^{57} \mathrm{Fe}$-enriched maghemite nanoparticles that is related to a deviation of about half of the iron atomic spins in both tetrahedral and octahedral sites from perfect ferrimagnetic order. Neutron diffraction is sensitive to the atomic magnetic moments (Golosovsky et al., 2001) and, applied to maghemite nanoparticles, revealed a slightly larger disorder on the octahedral $\mathrm{Fe}$ site (Yusuf et al., 2006). Specifically for magnetite and maghemite nanoparticles, the reduced magnetization has recently been associated with antiphase boundaries as observed using high resolution TEM and diffraction techniques (Wetterskog et al., 2013; Nedelkoski et al., 2017).

Enhancement of the magnetic properties of iron oxide nanoparticles was achieved by the introduction of a silica shell, resulting in a significant increase of $\eta_{m}$ and in a decrease of the lower magnetized surface region as compared to the noncoated particles (Lee et al., 2015). Similarly, magnetic nanocrystals embedded in silicate glasses were found to consist of a magnetic core with equal magnetic and nuclear size, surrounded by a nonmagnetic shell (Raghuwanshi et al., 2014, 2015).

Further progress in the application of magnetic SANS to the study of intraparticle magnetization includes the investigation of more complex magnetic morphologies such as multidomain nanoparticles consisting of aggregates of magnetic crystallites (Dennis et al., 2015; Bender et al., 2017), magnetic nanosponges (Bonini et al., 2008), or antiferromagnetic nanoparticles with magnetic shell arising from surface spin disorder (Manna et al., 2012). For core-shell particle systems, magnetic SANS allows one to access the different magnetic contributions in core and shell (Ijiri et al., 2005), and the potential spin disorder at core-shell interfaces.

\section{Interparticle correlations}

Interactions between magnetic nanoparticles typically originate in magnetic dipole-dipole, steric, or van der Waals 
interactions and can be induced by increased particle concentration or application of magnetic, electric, or flow fields. As a result, the evolving structure factor indicates in the first approximation the attractive and repulsive interparticle interactions, a scenario that limits the single-particle scattering and therefore the particle-matrix approach. Structures reported for dispersions of magnetic nanoparticles or ferrofluids range from short-range ordered aggregates via chainlike structures to pseudocrystalline ordering in concentrated ferrofluids upon application of a magnetic field. For short-range correlations, models such as hard spheres or sticky hard spheres can be applied in combination with different approaches for the structure factor $S(q)$ along with the form factor $F(q)$. The $I \propto F^{2}(q) S(q)$ approach is applied in many situations where a simple expression for $S(q)$ is plausible. If the characteristic properties of the single nanoparticles give rise to pseudocrystalline ordering, even classical Bragg-scattering patterns including superstructure reflections are observed on the 2D detector (see Fig. 16). Here the structural information (superlattice symmetry) can be derived directly from the reflection positions, i.e., without complex fitting of a particular structure factor.

Magnetic SANS has been widely applied to the investigation of structure formation and stabilization of ferrofluids. Avdeev et al. (2015) reported the importance of both carrier polarity and stabilization techniques for the stability of ferrofluids; the stability of sterically, electrostatically, and double-sterically stabilized ferrofluids has been reviewed in detail. In general, the higher the surfactant efficiency, the more stable is the system at surfactant excess (Petrenko et al., 2016). Double-layer stabilization enables the use of carrier media with high polarity such as water for ferrofluids otherwise dispersed in nonpolar solvents, however, at the expense of stability (Avdeev et al., 2006). Attractive interparticle interactions, revealed in sterically stabilized nonpolar benzene-based ferrofluids, can be directly related to the magnetoviscous properties. In comparison, for moderately polar pentanol-based ferrofluids repulsive interparticle interactions are observed without any magnetoviscous effect (MVE) (Avdeev and Aksenov, 2010).

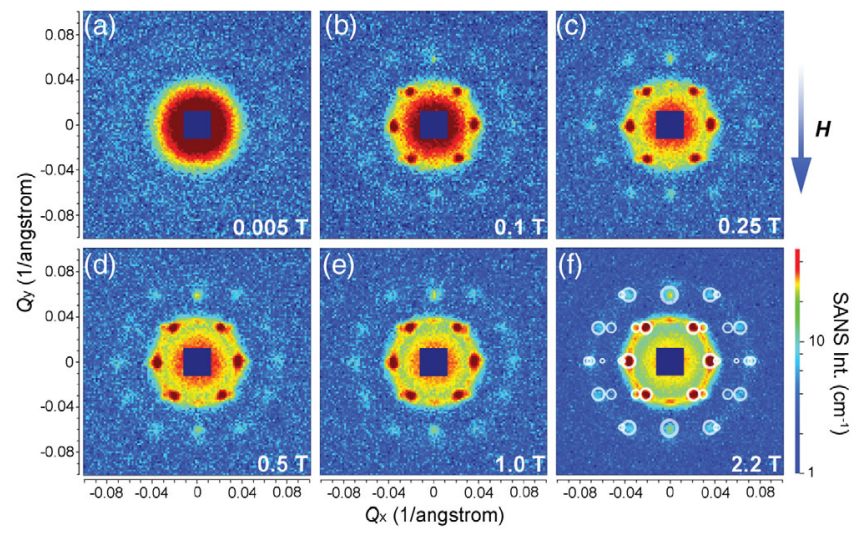

FIG. 16. SANS patterns of iron oxide nanoparticles in solution, exposed to external magnetic fields aligned vertically. (f) The calculated reflections of a face-centered cubic superstructure are shown as white circles. From Fu et al., 2016.
The MVE is related to an increased viscosity upon application of a magnetic field as a result of interparticle interactions in ferrofluids. Using magnetic RheoSANS, Pop and Odenbach established the correlation between the ferrofluid microstructure and the MVE as a result of aggregate or chain formation in the ferrofluid, whereas the macroscopically observed shear-thinning effect is induced by breakage of these chains upon increased shear rates (Pop et al., 2004; Pop and Odenbach, 2006). In the context of structural relaxation of magnetic nanoparticle aggregates, the potential of a rotating sample in a static magnetic field instead of using a rotating magnetic field was demonstrated for the investigation of the $q$-dependent diffusion time $\tau_{q}$, applicable to relaxation times of 3-300 ms (Wandersman et al., 2009). An analogous electroviscous effect was reported upon application of dc or ac electric fields, where changes in the viscosity were related to aggregate formation in transformer-oil-based ferrofluids (Rajnak et al., 2015, 2017; Kurimský et al., 2017).

Upon application of a magnetic field, strong anisotropy of the Brownian motion in solution is observed, resulting in a lowering of the concentration fluctuations along the field, concomitantly expressed by an anisotropic structure factor of an otherwise fluidlike magnetic colloid sample (Gazeau et al., 2002; Mériguet et al., 2006a, 2006b). Formation of chainlike structures that orient in the direction of an applied magnetic field was observed for magnetosomes (Hoell et al., 2004) as well as for cobalt nanoparticles (Barrett et al., 2011). On a larger scale, Jain et al. (2014) observed field-dependent chain formation of $160 \mathrm{~nm}$ ferrofluid emulsion droplets. Wiedenmann, Hoell, and Kammel (2002) discussed the fieldand concentration-induced structure formation in cobalt ferrofluids in detail; depending on the particle concentration, a transition of isolated particles $(<1 \mathrm{vol} \%)$ to short-range ordered aggregates ( $5 \mathrm{vol} \%$ ) is observed. Upon application of a magnetic field, a combination of chain-line structures and pseudocrystalline ordering is found (Wiedenmann et al., 2003; Wiedenmann and Heinemann, 2005; Heinemann, Wiedenmann, and Kammel, 2007). A SANSPOL study of colloidal magnetite nanoparticles illustrates how a larger particle size leads to larger dipole-dipole interaction, resulting in 2D nanoparticle sheets with hexagonal symmetry (Klokkenburg et al., 2007). Recently, Fu et al. (2016) reported the field-induced self-organization of iron oxide nanoparticles. The combined SANS and very small-angle neutron scattering (VSANS) study gave information on both the symmetry of the superlattice and its extensions in large-scale oriented aggregates. Whereas aggregate formation is already observed at a very low field of $0.02 \mathrm{~T}$, a highly crystalline superlattice emerges beyond $0.1 \mathrm{~T}$ that is indexed according to a fcc lattice with the nearest-neighbor direction [110] oriented parallel to the applied field (see Fig. 16).

In a solid matrix, liquidlike short-range order of small 2-nm-sized Fe nanoparticles has been identified, revealing ferromagnetic interparticle correlations at temperatures below 100 K (Bellouard, Mirebeau, and Hennion, 1996). Fielddependent SANS experiments show magnetic interparticle correlations in dense assemblies of $\mathrm{Fe}$ and $\mathrm{Co}$ nanoparticles with different degree of structural order (Ijiri et al., 2005; Farrell et al., 2006; Sachan et al., 2008). Ridier et al. (2017) 
recently revealed the individual-collective crossover depending on the particle size in dense assemblies of nanoparticles; the observation of ferromagnetically correlated clusters of $4.8 \mathrm{~nm}$ particles, with a temperature-dependent magnetic correlation length, is opposed to the superparamagnetic behavior of larger $8.6 \mathrm{~nm}$ particles and was attributed to the much larger surface anisotropy in the smaller nanoparticles. Applied to granular perpendicular recording media, SANSPOL measurements provide both the magnetic grain size, being smaller than the nuclear grain size, and the interparticle correlation distance (Lister et al., 2009) as well as the switching process upon magnetization reversal (Lister et al., 2010).

\section{Anisotropic nanostructures}

The application of magnetic SANS has further been expanded to anisometric magnetic nanoparticles such as oriented nanowires. Magnetic nanowires are nowadays routinely prepared using templated techniques with either amorphous silica or anodized alumina matrices as templates. The degree of alignment and order is tunable from 2D powders of aligned nanowires with short-range order toward crystalline arrangements with hexagonally packed nanowires. With typical dimensions of a few tens of $\mathrm{nm}$ in cross section, such structures still fit in the framework of nanomaterials (i.e., with two dimensions smaller than $\sim 100 \mathrm{~nm}$ ). Magnetization reversal in aligned nanowire arrays has been studied using both polarized and unpolarized SANS, and spin misalignment is commonly observed (Grigoryeva et al., 2007; Günther et al., 2014; Maurer et al., 2014; Grutter et al., 2017). However, with a typical nanowire length approaching the micrometer range and usually oriented parallel to the neutron beam, arrays of magnetic nanowires act as a grating, and strong multiple scattering has to be taken into account depending on the nanowire length; this effect is discussed in depth by Grigoriev et al. (2010). Because of their relatively large volume, nanowires exhibit stable ferromagnetic properties and are mostly multidomain structures. The particle-matrix approach is therefore not applicable to nanowires; indeed, micromagnetic simulations (Vivas, Yanes, and Michels, 2017) and experimental data (Günther et al., 2014) demonstrate strong deviations from the uniform-particle form-factor model.

\section{MICROMAGNETIC SIMULATIONS}

In this section we report on the progress made recently in the understanding of magnetic SANS data employing fullscale micromagnetic simulations. These studies take into account the full nonlinearity of Brown's static equations of micromagnetics, in contrast to the analytical calculations reviewed in Sec. II.C, which are limited to the approach-tosaturation regime.

\section{A. Novel micromagnetic simulation methodology for modeling bulk magnetic materials}

In polycrystalline bulk ferromagnets the sources of spin disorder are related to lattice imperfections, e.g., point defects, dislocations, or are directly associated with the polycrystalline nature of these materials, e.g., to grain and phase boundaries, or pores. These microstructural defects are accompanied by spatial variations of the materials parameters, for instance, the magnitude of the local saturation magnetization, exchange constant, or variations in the magnitude and/or direction of the magnetic anisotropy field. As a result, these features give rise to a deviation of the magnetization from the perfectly aligned state. Hence, they lead to spin misalignment and to an ensuing strong magnetic SANS signal.

In this section we review a novel micromagnetic simulation methodology which takes site-dependent magnetic parameters (saturation magnetization, magnetic anisotropy) and interactions (exchange and magnetodipolar fields) into account. This approach enables studies of the magnetic microstructure of a wide range of polycrystalline magnetic materials such as single-phase nanocrystalline magnets, magnetic nanocomposites, recording media, or magnetic particles in a nonmagnetic matrix (Löffler et al., 2005; Ogrin et al., 2006; Saranu et al., 2008; Erokhin et al., 2012a, 2012b; Zighem et al., 2013; Michels et al., 2014; Erokhin, Berkov, and Michels, 2015).

The majority of results which are discussed later on were obtained on nanocomposites. We emphasize that this class of materials is one of the most complicated from the point of view of numerical simulations. The main difficulty is that they consist of at least two phases, and the boundaries between these phases are complicated curved surfaces; a typical example is a hard-soft nanocomposite consisting of magnetically hard (i.e., having a large magnetocrystalline anisotropy) crystal grains surrounded by a magnetically soft matrix. In order to perform accurate and efficient simulations of multiphase nanocomposites, Erokhin et al. (2012a, 2012b) proposed a methodology which combines the advantages of a flexible mesh generation with an effective calculation of the micromagnetic energy. Namely, the whole mesh generation algorithm can be viewed (Michels et al., 2014) as a method to discretize a sample into polyhedra having nearly spherical shape. It allows the use of spherical dipole approximationequivalent to the point-dipole approximation-for the evaluation of the magnetodipolar interaction between the various finite elements.

In the micromagnetic simulations all four standard contributions to the total magnetic free energy are taken into account: energy in the external magnetic field, energy of the magnetocrystalline anisotropy, exchange stiffness, and magnetodipolar interaction energies (the antisymmetric Dzyaloshinski-Moriya interaction can also be implemented). The system energy due to the presence of an external magnetic field and the energy of the magnetocrystalline anisotropy (which can be uniaxial and/or cubic) are calculated in the model in the standard way, namely,

$$
\begin{gathered}
E_{\mathrm{ext}}=-\sum_{i=1}^{N} \boldsymbol{\mu}_{i} \cdot \mathbf{H}, \\
E_{\mathrm{an}}^{\mathrm{un}}=-\sum_{i=1}^{N} K_{i}^{\mathrm{un}} \Delta V_{i}\left(\mathbf{m}_{i} \cdot \mathbf{n}_{i}\right)^{2},
\end{gathered}
$$$$
E_{\mathrm{an}}^{\mathrm{cub}}=\sum_{i=1}^{N} K_{i}^{\mathrm{cub}} \Delta V_{i}\left(m_{i, x^{\prime}}^{2} m_{i, y^{\prime}}^{2}+m_{i, y^{\prime}}^{2} m_{i, z^{\prime}}^{2}+m_{i, x^{\prime}}^{2} m_{i, z^{\prime}}^{2}\right) \text {, }
$$ 
where $\mathbf{H}$ is the external field, $\boldsymbol{\mu}_{i}=\boldsymbol{\mu}\left(\mathbf{r}_{i}\right)$ and $\Delta V_{i}$ are the magnetic moment and the volume of the $i$ th finite element (polyhedron), and $\mathbf{m}_{i}=\boldsymbol{\mu}_{i} / \mu_{i}$ denotes the unit magnetization vector. Both the anisotropy constants $K_{i}$ and the directions of the anisotropy axes $\mathbf{n}_{i}$ can be site dependent, as required for a polycrystalline material. The symbols $m_{i, x^{\prime}}$ represent the components of the unit magnetization vectors in the local coordinate system that is attached to the cubic anisotropy axes. Higher-order anisotropy contributions can be taken into account.

The evaluation of the exchange-energy contribution in the model requires a much more sophisticated approach than in the standard finite difference method (FDM), because the continuous integral version of this energy contains magnetization gradients,

$$
E_{\mathrm{exch}}=\int_{V} A(\mathbf{r})\left[\left(\nabla m_{x}\right)^{2}+\left(\nabla m_{y}\right)^{2}+\left(\nabla m_{z}\right)^{2}\right] d V,
$$

where $A$ denotes the exchange-stiffness constant, and $V$ is the sample volume. Finding an approximation to Eq. (51) for a disordered system based on some interpolation procedure preserving the smooth behavior of magnetization componentsrequired for the correct evaluation of derivatives in Eq. (51) - is a highly complicated task.

Berkov and Gorn (2005) chose a completely different approach and developed an algorithm for the exchange-energy evaluation based on the summation of the nearest-neighbor contributions, widely used in FDMs. The integral in Eq. (51) is approximated by the following sum:

$$
E_{\text {exch }}=-\frac{1}{2} \sum_{i=1}^{N} \sum_{j \in \text { n.n. }(i)} \frac{2 A_{i j} \Delta V}{a^{2}}\left(\mathbf{m}_{i} \cdot \mathbf{m}_{j}\right) .
$$

Here $a$ is the cell size of a regular cubic grid (so that the cell volume is $\left.\Delta V=a^{3}\right), A_{i j}$ denotes the exchange-stiffness constant between cells $i$ and $j$, and the notation $j \subset$ n.n. $(i)$ means that the inner summation is performed over the nearest neighbors of the $i$ th cell only. We note that this Heisenberglike expression is valid only when the angles between neighboring moments are not too large; Berkov and Gorn (2005) showed that neglecting this condition can lead to unphysical results.

In the case of a disordered system, the following expression for the exchange-stiffness energy, which is analogous to Eq. (52), has been proposed:

$$
E_{\mathrm{exch}}=-\frac{1}{2} \sum_{i=1}^{N} \sum_{j \subset \mathrm{n} . \mathrm{n} .(i)} \frac{2 A_{i j} \Delta \bar{V}_{i j}}{\Delta r_{i j}^{2}}\left(\mathbf{m}_{i} \cdot \mathbf{m}_{j}\right),
$$

where $\Delta \bar{V}_{i j}=\left(\Delta V_{i}+\Delta V_{j}\right) / 2, \Delta r_{i j}$ is the distance between the centers of the $i$ th and the $j$ th finite elements with volumes $\Delta V_{i}$ and $\Delta V_{j}$, and $A_{i j}$ is the exchange constant. This expression should be corrected taking into account that the number of nearest neighbors for different finite elements may be different. It is done by the introduction of the correction factor $6 / n_{\mathrm{av}}$, where $n_{\mathrm{av}}$ is the average number of nearest neighbors for the particular random realization of the disordered finite element system. The accuracy of this simple correction method is surprisingly good, as shown by tests presented by Erokhin et al. (2012b) and Michels et al. (2014).

The energy of the long-range magnetodipolar interaction between magnetic moments and the corresponding contribution to the total effective field are computed using the pointdipole approximation as

$$
E_{\mathrm{dip}}=-\frac{1}{2} \sum_{i=1}^{N} \boldsymbol{\mu}_{i} \sum_{j \neq i} \frac{3 \mathbf{e}_{i j}\left(\mathbf{e}_{i j} \cdot \boldsymbol{\mu}_{j}\right)-\boldsymbol{\mu}_{j}}{\Delta r_{i j}^{3}},
$$

i.e., magnetic moments of finite elements are treated as point dipoles located at the polyhedra centers. This approximation is equivalent to the approximation of spherical dipoles, i.e., it would be exact for spherical finite elements. Hence, for the discretized system, this approximation introduces some computational errors, because the finite elements are polyhedra. However, these errors are small, because the shape of these polyhedra is close to spherical, due to the special algorithm employed for the generation of the mesh. If necessary, these errors can be significantly reduced further, taking into account higher-order terms in the multipole expansion.

The summation in Eq. (54) is performed by the particlemesh Ewald method. The specific implementation of the lattice-based Ewald method for the magnetodipolar interaction for regular and disordered systems of magnetic particles is described by Berkov and Gorn (1998) and Gorn et al. (2007). The major advantage is the possibility to use fast Fourier transform (FFT) for computing the long-range part of the total magnetodipolar field.

For the minimization of the total magnetic energy, obtained as the sum of all the contributions previously described, a highly optimized version of a gradient method is used employing the dissipation part of the Landau-Lifshitz equation of motion for magnetic moments (Landau and Lifshitz, 1935; Berkov, 2007). For the termination of the energy minimization, the local torque criterion is taken into account: the iteration process stops, if the maximal torque acting on magnetic moments is smaller than some prescribed value. This condition is more appropriate than the alternative criterion of a sufficiently small energy difference between two subsequent steps.

The methodology previously described was successfully employed (Erokhin et al., 2012a) for the explanation of the nontrivial SANS cross section (the so-called clover-leaf pattern) observed in the Fe-based nanocrystalline alloy NANOPERM (Suzuki and Herzer, 2006). This nanocomposite consists of magnetically hard grains (with a typical size of $12 \mathrm{~nm}$ ) surrounded by a soft magnetic matrix. Figure 17(a) demonstrates the "sample" used in micromagnetic simulations: each blue mesh element represents a hard crystallite with a homogeneous magnetization within it; warm colors mark mesh elements representing the soft phase. The approach permits the modeling of a relatively large sample volume $\left(250 \times 600 \times 600 \mathrm{~nm}^{3}\right)$, providing a high statistical accuracy of simulation results.

Further development (Erokhin and Berkov, 2017) of the simulation technique (Erokhin et al., 2012a, 2012b; Michels et al., 2014) was necessary to study the influence of the 

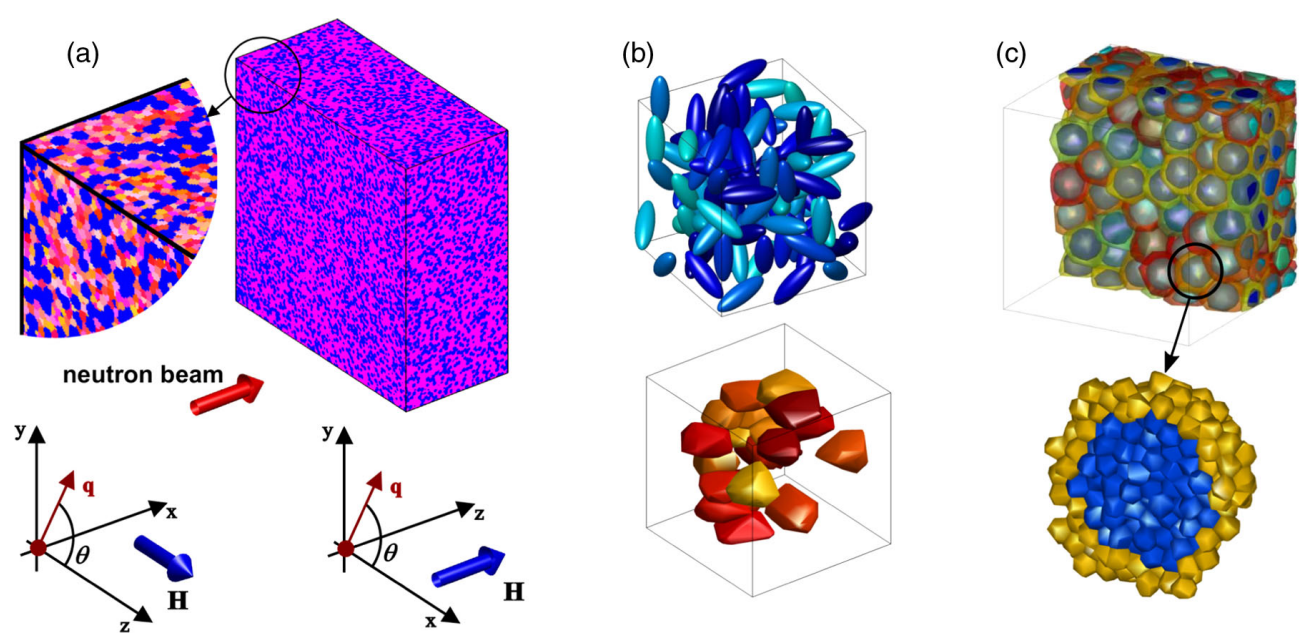

FIG. 17. (a) Schematics of the two scattering geometries and the microscopic structure of the nanocomposite sample with simulation volume $V=250 \times 600 \times 600 \mathrm{~nm}^{3}$. Blue polyhedrons: Fe particles; yellow, orange, and red polyhedrons: the matrix phase. From Michels et al., 2014. (b) Examples of the spatial distribution of hard magnetic crystallites (soft crystallites not shown) with different aspect ratios of corresponding ellipsoids of revolution. From Erokhin and Berkov, 2017. (c) Core-shell microstructure used in the micromagnetic simulations of a Nd-Fe-B nanocomposite and example of a polyhedron mesh-element distribution in a single grain; blue (yellow) elements correspond to the core (shell), typical mesh-element size is $2 \mathrm{~nm}$.

nonspherical shape of hard grains [Fig. 17(b)] on the magnetic behavior of nanocomposites. In this case, hard crystallites had to be discretized into smaller mesh elements, because the spherical dipole approximation for the magnetodipolar field created by hard grains was insufficient due to their nonspherical shape. Another example of the geometrical flexibility of the methodology is the recent micromagnetic modeling of a $\mathrm{Nd}-\mathrm{Fe}-\mathrm{B}$ nanocomposite, where a core-shell particle model for the description of 20-nm-sized Nd-Fe-B grains (Erokhin et al., 2018) is implemented. This model takes into account changes in magnetic parameters of the $\mathrm{Nd}-\mathrm{Fe}-\mathrm{B}$ crystallites near their surface, which may be imperfect due to the manufacturing process. A typical core-shell microstructure used in these simulations and a polyhedron mesh used for the discretization of a grain are shown in Fig. 17(c). In order to resolve the magnetization distribution inside the shell, the mesh-element size has been set to $2 \mathrm{~nm}$.

Several examples of micromagnetic simulation results for various structures-a nanocomposite of the NANOPERM type, magnetic nanoparticles of different sizes, or a core-shell structure of Nd-Fe-B-are collected in Fig. 18. In particular, Fig. 18(a) displays the spatial distribution of the magnetization component $\mathbf{M}_{\perp}$ perpendicular to the applied field around two $\mathrm{Fe}$ nanoparticles in NANOPERM along with the magnetodipolar field produced by these particles. Generally speaking, the sources of the magnetodipolar field are regions with a nonzero divergence of the magnetization $(\nabla \cdot \mathbf{M} \neq 0)$. For magnetic nanocomposites, the most prominent "magnetic volume charges" $\rho_{\text {mag }}=-\nabla \cdot \mathbf{M}$ are due to the abrupt changes in the magnetic materials parameters at the phase boundary between particles and matrix, e.g., variations in the magnetization or anisotropy constants. Such jumps in the magnetic material parameters may give rise to an inhomogeneous spin structure which decorates each nanoparticle. Exactly this situation, for a magnetization jump of $\Delta M=$ $1200 \mathrm{kA} / \mathrm{m} \hat{=} 1.5 \mathrm{~T}$ at the interface between the Fe particle and the amorphous magnetic matrix in NANOPERM, can be observed in Fig. 18(a), where correlations between the magnetodipolar field and the magnetization distribution in the soft phase can be clearly seen.

Another important question in the development of nanomaterials is the determination of the critical single-domain size for magnetic nanoparticles of various shapes. This parameter is crucial for the production of high-performance permanent magnets based on these materials. The system of small-sized magnetic grains behaves like a system of StonerWohlfarth particles (each having a homogeneous magnetization), resulting in a high remanence and large coercivity of a nanocomposite. By contrast, the vortex state formed inside larger particles with sizes above the single-domain limit results in a very small remanence and in a reduced coercive field [the magnetization distributions of both types are presented in Fig. 18(b)].

Advancement of permanent-magnet materials requires a clear physical understanding of the relation between their microstructure and the macroscopic magnetic properties. To establish this relation for the core-shell model of a Nd-Fe-B nanocomposite, Fig. 18(c) shows the magnetization distribution at selected points on the hysteresis curve (large positive field, remanence, coercivity). It can be clearly seen that in high fields the shells exhibit a larger magnetization projection in the field direction than the cores, since the shell anisotropy constants are reduced compared to the core regions. This situation prevails down to the remanent state, where a qualitatively similar spin distribution is observed. However, at negative fields the shells reverse their magnetization "easier" than the cores [cf., e.g., the dashed curves in Fig. 18(c)], which again can be attributed to the reduced anisotropy in the shell region. The most interesting feature of the presented hysteresis is the contradiction between a relatively low coercivity and a "hard" system behavior at large positive fields, where the saturation is not reached even 
(a)

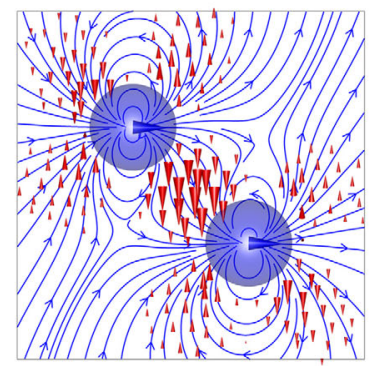

(b)

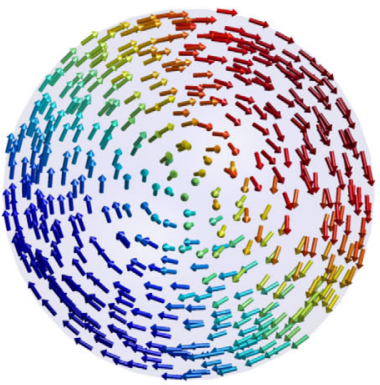

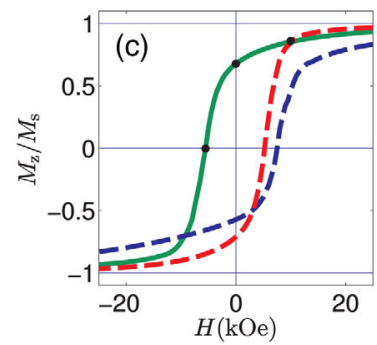

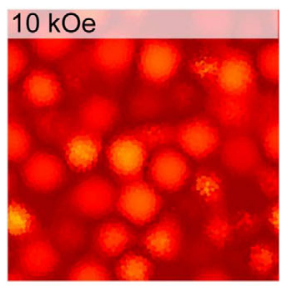

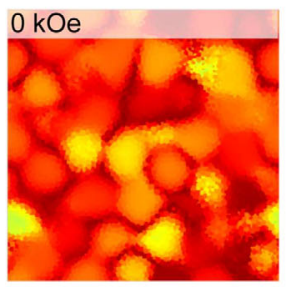

$-1$

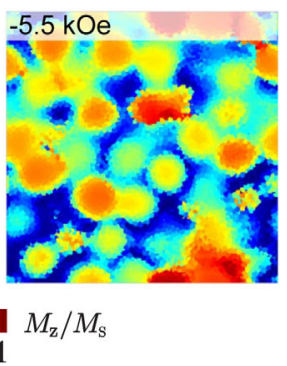

FIG. 18. (a) Results of a micromagnetic simulation for the spin distribution around two selected Fe nanoparticles (blue circles), which are assumed to be in a single-domain state. The external magnetic field $\mathbf{H}$ is applied horizontally in the plane. The magnetization component $\mathbf{M}_{\perp}$ perpendicular to $\mathbf{H}$ is shown by red arrows; the thickness of the arrows is proportional to the magnitude of $\mathbf{M}_{\perp}$. Blue lines visualize the magnetodipolar field. From Michels et al., 2014. (b) Dependence of the spin structure of a nanoparticle on the particle size represented by selected magnetization configurations of Fe particles with diameters of $D=20$ and $40 \mathrm{~nm}$ at an external field of -30 Oe. (c) Simulated upper part of the hysteresis loop (green line) and magnetization distribution (two-dimensional cuts out of threedimensional distributions) at selected points on the hysteresis curve (approach to saturation, remanence, coercivity) obtained in the coreshell model of a Nd-Fe-B nanocomposite. Contributions to the hysteresis loop only from cores (blue dashed line) and shells (red dashed line) are also shown as lower parts of this loop.

at the applied field of $2 \mathrm{~T}$ (we call it the "hard-soft effect"). Erokhin et al. (2018) were able to explain this effect by combining the core-shell model of nanograins with the influence of a considerable volume fraction of particles in a superparamagnetic state.

\section{B. Simulation of magnetic neutron scattering: Decrypting SANS cross sections}

This section discusses simulation results for the magnetic SANS cross sections of soft magnetic nanocomposites. The most important advantage of numerical simulations is the possibility to decrypt the magnetic SANS cross sections, i.e., one can study the contribution of each individual Fourier component of the magnetization distribution separately. Also the twofold impact of the magnetodipolar interaction can be disentangled, which provides fundamental insights into magnetic SANS from polycrystalline materials (see Fig. 22).

Since the focus is on magnetic spin-misalignment scattering, the nuclear SANS contribution was ignored and the discussion was restricted to unpolarized neutrons. Note, however, that for polycrystalline texture-free magnetic nanocomposites the nuclear SANS signal is isotropic and independent of the applied magnetic field, and its magnitude is generally small compared to the spin-misalignment scattering discussed here (Michels and Weissmüller, 2008).

In the following we show selected results for the unpolarized magnetic SANS cross section $d \Sigma_{M} / d \Omega$ in the perpendicular scattering geometry [cf. Eq. (12) in
Sec. II.B]. The functions $M_{x, y, z}(\mathbf{r})$ are obtained from the micromagnetic simulations; applying the Fourier transformation and combining them into Eq. (12) (or another one for a different scattering geometry), we derive the simulated magnetic SANS cross section. In order to compare the numerical results with experiment, it is necessary to obtain the scattering cross section in the plane of the detector. We apply threedimensional Fourier transformation to $M_{x, y, z}(\mathbf{r})$ and use data on the plane $\left(q_{y}, q_{z}\right)$ at $q_{x}=0$, which corresponds to the standard SANS setup, where $q_{x}$ is negligible in comparison to the other two components.

Returning to the simulations of NANOPERM previously discussed, Fig. 19 displays projections of the functions $\left|\widetilde{M}_{x}\right|^{2}$, $\left|\widetilde{M}_{y}\right|^{2}$, and $\left|\widetilde{M}_{z}\right|^{2}$, and the cross term $C T=-\left(\widetilde{M}_{y} \widetilde{M}_{z}^{*}+\right.$ $\left.\widetilde{M}_{y}^{*} \widetilde{M}_{z}\right)$ present in Eq. (12) into the detector plane at selected external-field values. Also, the field dependence of the magnetic SANS cross section $d \Sigma_{M} / d \Omega$ is shown. This representation emphasizes the power of the approach: by employing numerical micromagnetics for the computation of magnetic SANS cross sections, it becomes possible to study the individual magnetization Fourier components and their contribution to $d \Sigma_{M} / d \Omega$. In particular, the approach of combining micromagnetic and SANS simulations complements experiments, which provide a weighted sum of Fourier components, a fact which often hampers the straightforward interpretation of experimental SANS data. While it is in principle possible to determine some Fourier coefficients separately, e.g., through the application of a saturating 

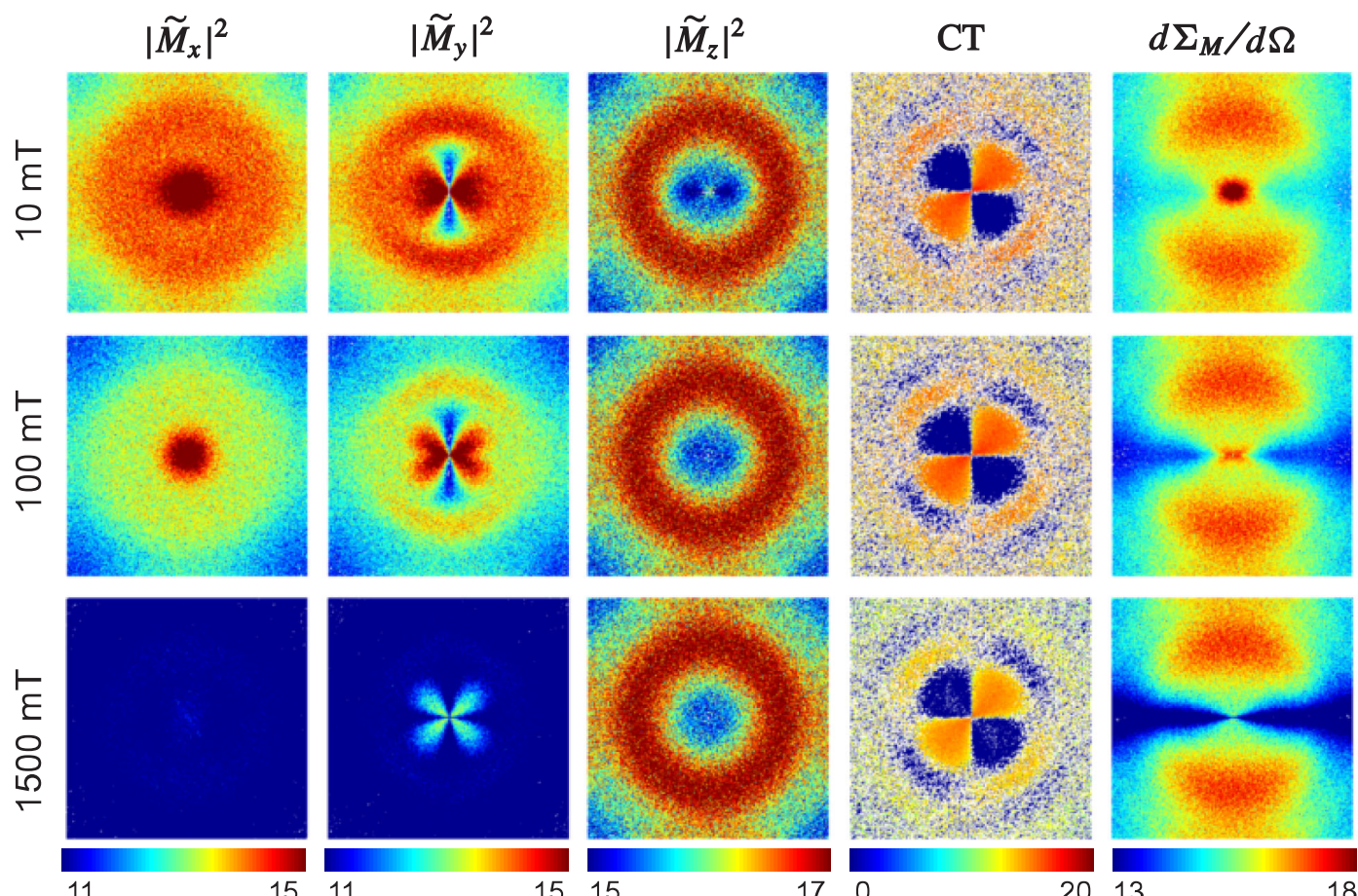

FIG. 19. Results of micromagnetic simulations for the Fourier components of the magnetization. The images represent projections of the respective functions into the detector plane $q_{x}=0$ for $\mathbf{k}_{0} \perp \mathbf{H}$. Columns from left to right: $\left|\widetilde{M}_{x}\right|^{2},\left|\widetilde{M}_{y}\right|^{2},\left|\widetilde{M}_{z}\right|^{2}$, $C T=-\left(\widetilde{M}_{y} \widetilde{M}_{z}^{*}+\widetilde{M}_{y}^{*} \widetilde{M}_{z}\right)$, and $d \Sigma_{M} / d \Omega$. Pixels in the image corners correspond to $q \cong 1.8 \mathrm{~nm}^{-1}$. A logarithmic color scale is used (color bars are in arbitrary units).

magnetic field or by exploiting the neutron-polarization degree of freedom via SANSPOL or POLARIS methods [see, e.g., Honecker et al. (2010) and Wiedenmann (2010)], it is difficult to unambiguously determine a particular scattering contribution without "contamination" by unwanted Fourier components. For instance, when the applied field is not large enough to completely saturate the sample, then the scattering of unpolarized neutrons along the field direction does not represent the pure nuclear SANS, but contains also the magnetic SANS due to the misaligned spins (Bischof et al., 2007).

Analyzing the individual contributions to the total magnetic SANS cross section presented in Fig. 19, we can see that $\left|\widetilde{M}_{z}\right|^{2}$ is nearly isotropic (i.e., $\theta$ independent) over the whole ranges of the applied field and scattering vectors. By contrast, at the smallest $q$ and largest fields, the Fourier coefficient $\left|\widetilde{M}_{y}\right|^{2}$ reveals a pronounced angular anisotropy with maxima roughly along the diagonals of the detector (the so-called clover-leaf anisotropy), whereas at the smaller fields, the anisotropy of $\left|\widetilde{M}_{y}\right|^{2}$ is rather of the $\cos ^{2} \theta$ type (i.e., elongated parallel to $\mathbf{H})$. At saturation $\left(\mu_{0} H=1.5 \mathrm{~T}\right)$, both $\left|\widetilde{M}_{x}\right|^{2}$ and $\left|\widetilde{M}_{y}\right|^{2}$ are relatively small and the main contribution to $d \Sigma_{M} / d \Omega$ is due to the term $\left|\widetilde{M}_{z}\right|^{2}$, which originates from nanoscale jumps of the magnetization at phase boundaries. By decreasing the field, the magnitude of the transversal components increases as the spin misalignment on the scale of tens of nanometers develops. The $C T$ changes its sign between the detector quadrants: it is positive for $0^{\circ}<\theta<90^{\circ}$, negative for $90^{\circ}<\theta<180^{\circ}$, and so on. When the $C T$ is multiplied by $\sin \theta \cos \theta$, the corresponding contribution to the total $d \Sigma_{M} / d \Omega$ becomes positive for all angles $\theta$. Therefore, and contrary to the common assumption that the $C T$ averages to zero for statistically isotropic polycrystalline microstructures, the $C T$ appears to be of a special relevance in nanocomposite magnets. Note that the symmetry of the $C T$ replicates the symmetry of the spin structure [compare the fourth column in Fig. 19 to Fig. 18(a)]. In the presence of an applied magnetic field the stray-field distribution and the associated magnetization configuration around each nanoparticle are qualitatively similar (on the average), thus giving rise to dipolar correlations which add up to a positive-definite $C T$ contribution to $d \Sigma_{M} / d \Omega$.

The angular dependence of $\left|\widetilde{M}_{x}\right|^{2}$ (Fig. 19) might give the impression that this component is isotropic, but this is the case only in the plane $q_{x}=0$. Figures 20(a) and 20(b) contain a three-dimensional picture of the Fourier components derived from the spatial magnetization distribution. It turns out that $\left|\widetilde{M}_{x}\right|^{2}$ is not only strongly anisotropic at larger $q_{x}$ [Fig. 20(a)], but also exhibits the clover-leaf pattern [Fig. 20(b)] in the plane $\left(q_{x}, q_{z}\right)$. Indeed, $\left|\widetilde{M}_{x}\right|^{2}\left(q_{x}, q_{y}, q_{z}\right)$ and $\left|\widetilde{M}_{y}\right|^{2}\left(q_{x}, q_{y}, q_{z}\right)$ coincide with respect to the rotation around the $q_{z}$ axis, because the only symmetry breaking in the system is due to the external magnetic field.

The next important insight into the field-dependent relation between the different contributions in Eq. (12) can be deduced from the radially averaged data shown in Fig. 20(c). For the particular applied field of $\mu_{0} H=30 \mathrm{mT}$ used for this figure, the dominance of $\left|\widetilde{M}_{x}\right|^{2}$ and $\left|\widetilde{M}_{y}\right|^{2}$ in the small $q$ range becomes evident, especially when the logarithmic scale of the cross-section axis is considered. By contrast, at larger $q$ the 
(a)

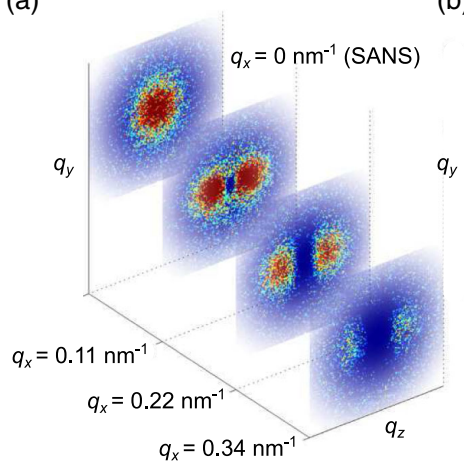

(b)

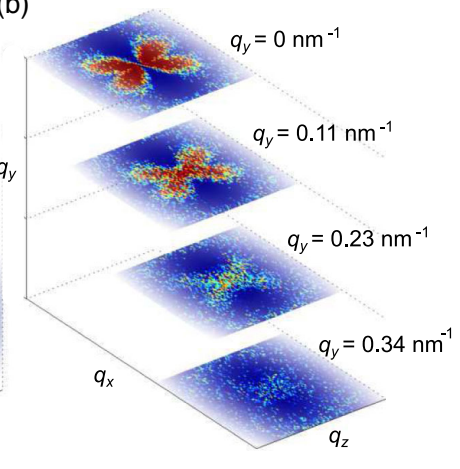

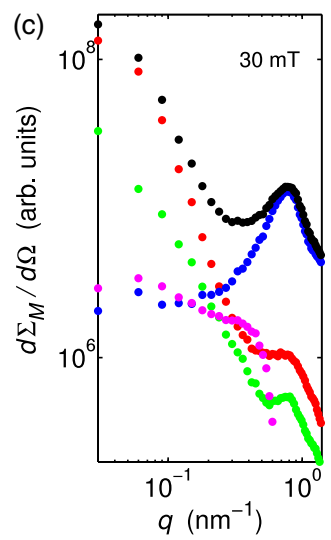

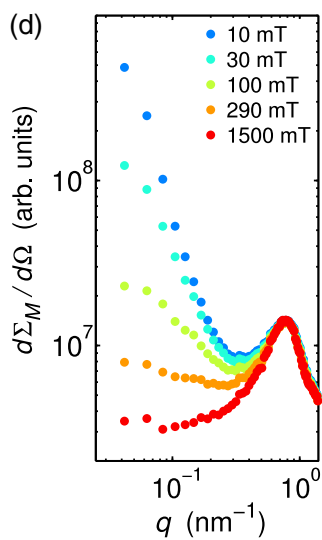

FIG. 20. (a), (b) Two-dimensional cuts (shifted for a better visibility) of $\left|\widetilde{M}_{x}\right|^{2}\left(q_{x}, q_{y}, q_{z}\right)$. (c) Radially averaged individual scattering contributions to $d \Sigma_{M} / d \Omega$ as a function of the scattering vector $q\left(\mathbf{k}_{0} \perp \mathbf{H}\right)\left(\log -\log\right.$ scale): total $d \Sigma_{M} / d \Omega(\mathrm{black}) ;\left|\widetilde{M}_{z}\right|^{2}$ sin ${ }^{2} \theta$ (blue); $C T$ (magenta); $\left|\widetilde{M}_{y}\right|^{2} \cos ^{2} \theta$ (green); $\left|\widetilde{M}_{x}\right|^{2}$ (red). (d) Radially averaged total magnetic SANS cross section $d \Sigma_{M} / d \Omega$ as a function of the scattering vector $q$ and for several applied magnetic fields $H$ (see the inset) $\left(\mathbf{k}_{0} \perp \mathbf{H}\right)(\log -\log$ scale). Materials parameters for NANOPERM were used.

influence of the $\left|\widetilde{M}_{z}\right|^{2}$ component on the resulting cross section prevails.

Figure 20(d) is an illustration of the strong dependence of magnetic SANS on the applied magnetic field. In the small $q$ range, the difference between the cross section at $\mu_{0} H=$ $10 \mathrm{mT}$ and at saturation $(1500 \mathrm{mT})$ can be as large as 2 orders of magnitude. This observation demonstrates the tremendous sensitivity of SANS in the study of magnetic materials; particularly, it has a special importance for the investigation of magnetization distributions of such an extremely magnetically soft nanocomposite as NANOPERM, where the difference of total magnetizations at the applied fields of 10 and $1500 \mathrm{mT}$ does not exceed 5\%. The field-independent local maximum at $q \cong 0.78 \mathrm{~nm}^{-1}$ corresponds to $\sim 8 \mathrm{~nm}$ distance in the real space and agrees well with the size of the hard magnetic crystallites used in the model.

The finding that $\left|\widetilde{M}_{z}\right|^{2}$ is nearly isotropic and that $\left|\widetilde{M}_{y}\right|^{2}=$ $\left|\widetilde{M}_{y}\right|^{2}(q, \theta)$ strongly depends on the angle $\theta$ provides a straightforward explanation for the experimental observation of the clover-leaf anisotropy in the SANS cross section of the nanocrystalline two-phase alloy NANOPERM (Michels et al., 2006). The simulation results for the difference cross section, i.e., where the scattering at saturation $\left(\mu_{0} H=1.5 \mathrm{~T}\right)$ has been subtracted, agree semiquantitatively (up to an unknown scaling factor) with the experimental data, as shown in Fig. 21 (Erokhin et al., 2011, 2012b). Clover-leaf-type anisotropies in $d \Sigma_{M} / d \Omega$ have also been reported for a number of other materials, including precipitates in steels (Bischof et al., 2007), nanocrystalline Gd (Michels et al., 2008; Döbrich et al., 2012), and nanoporous Fe (Michels et al., 2009).

As a final point, the impact of the magnetodipolar interaction is discussed: the quantity of interest in an elastic magnetic neutron scattering experiment, the differential scattering cross section $d \Sigma_{M} / d \Omega$, depends in a twofold manner on this interaction. First, the interaction of the magnetic moment of the neutron with the sample's magnetization results in dipolar selection rules which are embodied, e.g., by the appearance of trigonometric functions in $d \Sigma_{M} / d \Omega$ [via the Halpern-Johnson vector, Eq. (7)] (Squires, 1978). Second, the magnetodipolar interaction between the magnetic moments in the sample has a direct impact on its magnetization configuration and therefore on the Fourier components of the magnetization. The former determine the properties of $d \Sigma_{M} / d \Omega$ and the latter the total magnetization (as measured by magnetometry).

The corresponding difference can be demonstrated by comparing the results for the correlation function of the spin-misalignment SANS cross section (Michels et al., 2003; Weissmüller et al., 2004; Döbrich et al., 2012; Mettus and Michels, 2015),

$$
C_{M}(y, z) \sim \int \frac{d \Sigma_{M}}{d \Omega}\left(q_{y}, q_{z}\right) e^{i \mathbf{q} \cdot \mathbf{r}} d^{2} q
$$
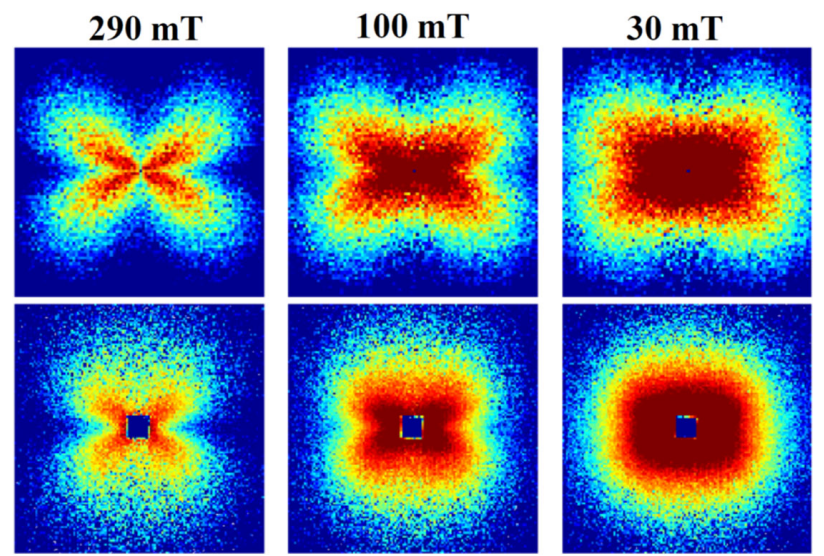

FIG. 21. Comparison between simulated (upper row) and experimental (lower row) data for the difference cross section $\propto\left(\left|\widetilde{M}_{x}\right|^{2}+\left|\widetilde{M}_{y}\right|^{2} \cos ^{2} \theta+C T \sin \theta \cos \theta\right)$ for various external fields as indicated $\left(\mathbf{k}_{0} \perp \mathbf{H}\right)$. Pixels in the image corners correspond to $q \cong 0.64 \mathrm{~nm}^{-1}$. A logarithmic color scale is used. Since experimental data were not obtained in absolute units, their values are scaled by a constant factor for comparison with simulated data. $\mathbf{H}$ is horizontal in the figure plane. From Michels et al., 2014. 
with the autocorrelation function $C_{S M}(\mathbf{r})$ of the magnetization component perpendicular to the applied field; the latter is defined as

$$
C_{S M}(\mathbf{r}) \sim \int \mathbf{M}_{\perp}(\mathbf{x}) \cdot \mathbf{M}_{\perp}(\mathbf{x}+\mathbf{r}) d^{3} x
$$

and is not decorated by the dipolar interaction between the neutrons and the sample magnetization. Using the convolution theorem, Eq. (56) can be rewritten as

$$
C_{S M}(\mathbf{r}) \sim \int\left(\left|\widetilde{M}_{x}(\mathbf{q})\right|^{2}+\left|\widetilde{M}_{y}(\mathbf{q})\right|^{2}\right) e^{i \mathbf{q} \cdot \mathbf{r}} d^{3} q
$$

Both correlation functions, simulated for porous $\mathrm{Fe}$, are depicted at a field of $\mu_{0} H=0.6 \mathrm{~T}$ in Fig. 22 along the horizontal $(z)$ and vertical $(y)$ directions; see Fig. 6, which depicts the spin structure around a spherical pore in Fe. One recognizes the existence of anisotropic correlations already for the autocorrelation function of the spin misalignment (not influenced by the interaction between neutrons and magnetic moments), which may be expected due to the long-range and anisotropic nature of the magnetodipolar interaction. The difference between both directions is significant (in particular for $r \cong 30-40 \mathrm{~nm}$ ) with $C_{S M}$ along the vertical direction being exclusively positive, while $C_{S M}$ along the horizontal direction intersects the $r$ axis at $r \cong 20 \mathrm{~nm}$ and possesses a minimum at $r \cong 30 \mathrm{~nm}$.

The existence of "anticorrelations" in $C_{S M}$ around these particular $r$ values is a manifestation of the typical magnetization distribution $\mathbf{M}_{\perp}(\mathbf{r})$ around a pore (see Fig. 6), which is due to the configuration of the magnetodipolar field in the
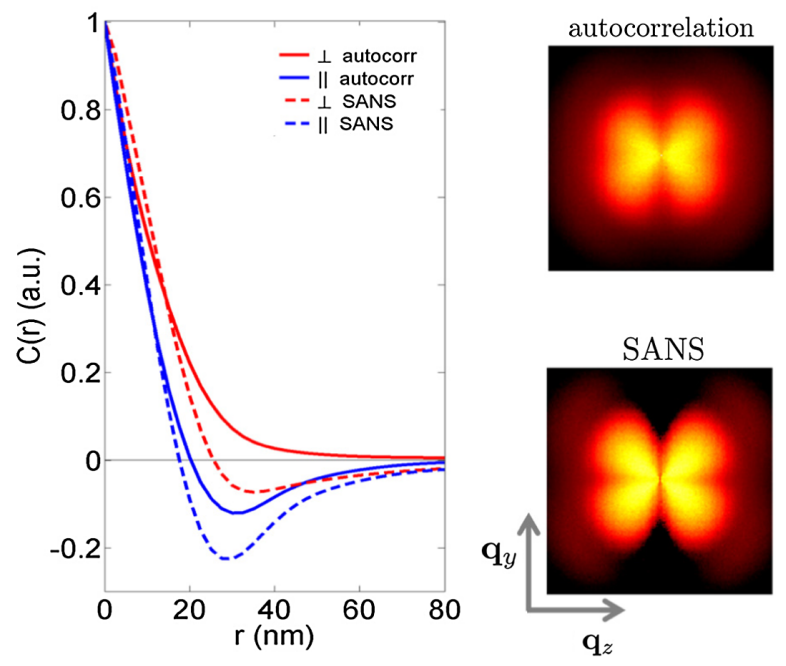

FIG. 22. Comparison between the normalized autocorrelation function of the spin misalignment $C_{S M}$ (solid lines) and the normalized correlation function of the spin-misalignment SANS cross section $C_{M}$ (dashed lines) along different directions in the $y-z$ detector plane $\left(\mu_{0} H=0.6 \mathrm{~T}\right.$ along $z$ ). The right images show the corresponding combination of Fourier components, projected into the detector plane: (autocorrelation) $\left|\widetilde{M}_{x}\right|^{2}+\left|\widetilde{M}_{y}\right|^{2}$; (SANS) $\left|\widetilde{M}_{x}\right|^{2}+\left|\widetilde{M}_{y}\right|^{2} \cos ^{2} \theta-\left(\widetilde{M}_{y} \widetilde{M}_{z}^{*}+\widetilde{M}_{y}^{*} \widetilde{M}_{z}\right) \sin \theta \cos \theta$. Pixels in the image corners correspond to $q \cong 0.4 \mathrm{~nm}^{-1}$. A logarithmic color scale is used. From Erokhin, Berkov, and Michels, 2015. vicinity of such an inclusion. Namely, the perpendicular magnetization component changes its sign along the direction of the applied field at a distance comparable with the pore diameter. Of course, the zeros and global minima of the correlation functions are dependent on the applied-field value.

Summarizing this section, we conclude that micromagnetic modeling perfectly complements magnetic SANS in decrypting the corresponding cross section by splitting it into different components or by providing the real-space magnetization distribution more appropriate for the physical analysis. As mentioned, magnetic SANS and micromagnetic simulations allow the investigation of the properties of materials in bulk; therefore, their powerful combination will provide fundamental insight into the magnetism of nanocomposites.

\section{SANS AS A PROBE OF NANOSCALE MAGNETIC INHOMOGENEITY IN COMPLEX MAGNETIC SYSTEMS}

\section{A. Magnetic inhomogeneity and magnetic phase separation}

Sections III and IV focus on systems with explicit chemical heterogeneity or assemblies of nanoparticles. There are, however, nominally chemically uniform materials that spontaneously nanostructure magnetically; these are the focus of this section. A simple paradigm in condensed matter physics is that materials with spatial homogeneity in structure and chemistry can be expected to exhibit corresponding homogeneity in properties. The complex, chemically disordered materials in many contemporary research fields stretch this paradigm to its limits, however, in some cases violating it. One important example emerged from the study of perovskite manganites that began in the mid-1990s, stimulated by the discovery of colossal magnetoresistance (CMR) (Coey, Viret, and von Molnár, 1999; Tokura and Tomioka, 1999; Dagotto, Hotta, and Moreo, 2001; Dagotto, 2002). Although these compounds were studied 40 years earlier (Jonker and Van Santen, 1950), their tendency to display large resistivity decreases in applied magnetic fields was overlooked. Originally identified in systems such as mixed-valence $\mathrm{La}_{1-x} \mathrm{Sr}_{x} \mathrm{MnO}_{3}, \mathrm{CMR}$ was eventually demonstrated to reach MR ratios of $10^{12}$ in just a few tesla, justifying the term "colossal" (Coey, Viret, and von Molnár, 1999; Tokura and Tomioka, 1999; Dagotto, Hotta, and Moreo, 2001; Dagotto, 2002).

From the mid-1990s it was gradually understood that a feature of such materials, on both nanoscopic and microscopic scales, is inhomogeneity in quantities such as conductance and magnetization, even in samples of the highest structural or chemical quality (Dagotto, Hotta, and Moreo, 2001; Dagotto, 2002). Evidence for this was accumulated from macroscopic measurements (e.g., magnetotransport, magnetometry, heat capacity), spectroscopic signatures of coexisting electronic or magnetic phases [e.g., from nuclear magnetic resonance (NMR)], imaging with scanning tunneling microscopy or spectroscopy (STM or STS), magnetic force microscopy and TEM, and reciprocal space methods such as neutron diffraction (ND) and inelastic neutron spectroscopy (INS) (Dagotto, Hotta, and Moreo, 2001; Dagotto, 2002). Real-space examples are provided in Figs. 23(a) and 23(b). Classic cases include nanoscale ferromagnetic metallic (FMM) clusters in a 

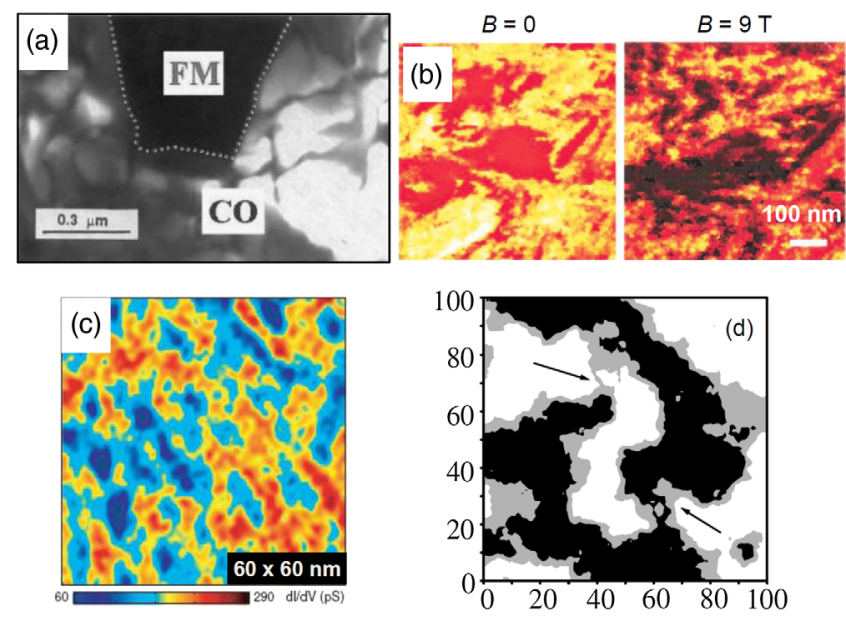

FIG. 23. Images of electronic and magnetic inhomogeneities in complex oxides. (a) Dark-field transmission electron microscope image of $\mathrm{La}_{0.25} \mathrm{Pr}_{0.38} \mathrm{Ca}_{0.38} \mathrm{MnO}_{3}$ at $20 \mathrm{~K}$ using a charge-ordered superlattice peak for contrast. The upper dark region is a chargedisordered ferromagnetic metallic region, while the lower textured region is a charge-ordered insulating region. From Cheong et al., 2002. (b) Scanning tunneling spectroscopy images of a $\mathrm{La}_{0.7} \mathrm{Ca}_{0.3} \mathrm{MnO}_{3}$ film just below its Curie temperature in 0 and 9 T. The color scale depicts local conductance, black being metallic, white insulating. Note the percolating ferromagnetic metallic region in the $9 \mathrm{~T}$ image. From Fath et al., 1999. (c) Scanning tunneling spectroscopy of $\mathrm{Bi}_{2} \mathrm{Sr}_{2} \mathrm{CaCu}_{2} \mathrm{O}_{8+\delta}$ in the normal state at $93 \mathrm{~K}$. The color scale depicts local conductance. Note the nanoscale heterogeneity. From Pasupathy et al., 2008. (d) Finite temperature Monte Carlo simulation from a random-field Ising model designed to describe manganites. $100 \times 100$ sites are shown, black and white corresponding, crudely, to ferromagnetic metal and nonferromagnetic insulator, respectively. From Mayr et al., 2001.

matrix of paramagnetic insulator around the Curie temperature $\left(T_{C}\right)$ in $\mathrm{La}_{0.7} \mathrm{Ca}_{0.3} \mathrm{MnO}_{3}$ [Fig. 23(b)] (Fath et al., 1999), and microscale coexistence of FMM and charge- and orbitally ordered antiferromagnetic insulator (COOAFI) phases in $\mathrm{La}_{0.5} \mathrm{Ca}_{0.5} \mathrm{MnO}_{3}$ and $\left(\mathrm{La}_{1-y} \mathrm{Pr}_{y}\right)_{1-x} \mathrm{Ca}_{x} \mathrm{MnO}_{3}$ [Fig. 23(a)] (Uehara et al., 1999; Cheong et al., 2002). These competing phases derive from the competition between spin, charge, orbital, and lattice degrees of freedom, metallic phases being favored by double exchange, insulating ones by charge order (CO), orbital order, and superexchange antiferromagnetism (AF) (Coey, Viret, and von Molnár, 1999; Tokura and Tomioka, 1999; Dagotto, Hotta, and Moreo, 2001; Dagotto, 2002). This competition was eventually understood in detail, the global manganite phase diagram being mapped in the plane of electronic bandwidth (ionic radius) versus quenched disorder (radius variance) (Tomioka and Tokura, 2004). The spatial variation in electronic and magnetic properties was termed electronic or magnetic inhomogeneity, or, as it often emerges from a uniform paramagnet on cooling, electronic or magnetic phase separation. These electronic inhomogeneities also occur in other oxides, including cobaltites and cuprates [Fig. 23(c)] (Pasupathy et al., 2008).

Great effort was expended on understanding the origin of this inhomogeneity, due to its role in CMR. Specifically, numerous studies concluded that CMR could be understood in terms of magnetic-field-induced coalescence of FMM regions in a non-FMM matrix [see, e.g., Fig. 23(b)] (Dagotto, Hotta, and Moreo, 2001; Dagotto, 2002). Models based on finite temperature double exchange, or double exchange competing with a superexchange AF insulator, among others, were shown capable of reproducing phase separation [see, e.g., Fig. 23(d)] (Mayr et al., 2001), even semiquantitatively reproducing the temperature $(T)$ and field $(H)$ dependence of the resistivity (Dagotto, Hotta, and Moreo, 2001; Dagotto, 2002). While subtleties remain, including two forms of CMR (Tokura et al., 1996) and two forms of phase separation [at nanoscales and microscales (compare Figs. 23(a) and 23(b)], this explanation for CMR has become widely accepted (Dagotto, Hotta, and Moreo, 2001; Dagotto, 2002). The role of disorder versus purely electronically driven phase separation remains a challenge, however, as does the understanding of chemical and structural inhomogeneities accompanying and/or driving the magnetic and electronic inhomogeneities.

Importantly, SANS played, and continues to play, an important role in detecting and elucidating such magnetic inhomogeneity in complex oxides. The ability to probe nanoscopic and mesoscopic scales, the high sensitivity to inhomogeneity, the magnetic sensitivity, and the penetration capability make SANS a powerful probe of this physics. Polarization, and measurement over a wide $T$ range, further enable separation of magnetic and structural and chemical contrasts. Methods and approaches originally honed on systems such as manganites have also now been applied to other complex oxides, as well as nonoxidic systems, including alloys. This section provides a summary of the application of SANS to the study of short-range magnetic inhomogeneity in these complex systems.

\section{B. Complex magnetic oxides}

As discussed, complex oxides are systems in which nanoscale and microscale magnetic inhomogeneities are especially important. We thus begin with a review of the use of SANS in the study of such materials, first in manganites (Sec. VII.B.1), and then in cobaltites (Sec. VII.B.2).

\section{Perovskite manganites}

As discussed in Sec. II, the neutron scattering intensity near $q=0$ is inherently sensitive to long-range FM order, often detected in elastic SANS via Porod scattering from magnetic domains. Such scattering follows Eq. (28), where $\Delta \eta$ arises due to magnetic contrast at domain walls and the power of 4 can be generalized to an exponent $n$. This equation is valid for $q \gg 2 \pi / D$, where $D$ is the size of the scattering object. For an FM these objects can be nonmagnetic (e.g., grains or other extended defects) or magnetic (typically long-range FMordered domains), $q \gg 2 \pi / D$ often being satisfied even at the lowest $q$ due to the large magnetic domains. The case $n=4$ [i.e., $d \Sigma / d \Omega \propto q^{-4}$, the "Porod law," Eq. (28)] is particularly common, describing scattering from 3D objects with "smooth" surfaces (Willis and Carlile, 2009). "Rough" or "wrinkled" surfaces result in $n<4, n=3$ marking the transition between surface and volume fractals (Kreyssig et al., 2009). In FMs, nonmagnetic contributions to Eq. (28) are often $T$ independent 
while magnetic ones are strongly $T$ dependent, making separation of magnetic and nonmagnetic scattering facile, even without polarization. An example is shown in
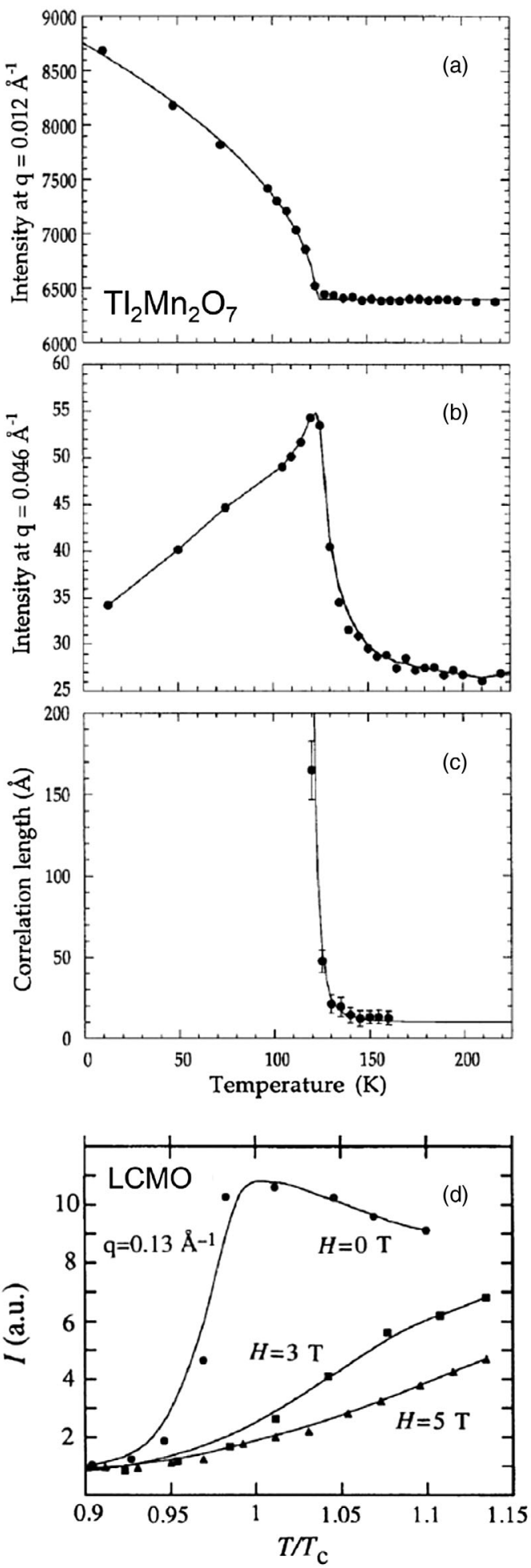

FIG. 24. Temperature dependence of (a) the $q=0.012 \AA^{-1}$ SANS intensity, (b) the $q=0.046 \AA^{-1}$ SANS intensity, and (c) the magnetic correlation length from polycrystalline $\mathrm{Tl}_{2} \mathrm{Mn}_{2} \mathrm{O}_{7}$. From Lynn, Vasiliu-Doloc, and Subramanian, 1998. (d) The temperature dependence (normalized to the Curie temperature) of the magnetic SANS intensity in polycrystalline $\mathrm{La}_{2 / 3} \mathrm{Ca}_{1 / 3} \mathrm{MnO}_{3}$ (LCMO) in three magnetic fields. From De Teresa et al., 1997.
Fig. 24(a) from the manganite pyrochlore $\mathrm{Tl}_{2} \mathrm{Mn}_{2} \mathrm{O}_{7}$, where the low- $q$ scattering (which follows the $n=4$ Porod law) clearly reflects the development of FM order, with $T_{C} \approx 120 \mathrm{~K}$ (Lynn, Vasiliu-Doloc, and Subramanian, 1998).

At a higher $q$ of $0.046 \AA^{-1}$ [Fig. 24(b)], $\mathrm{Tl}_{2} \mathrm{Mn}_{2} \mathrm{O}_{7}$ reveals a different $T$ dependence of the SANS intensity, referred to as critical scattering (Lynn, Vasiliu-Doloc, and Subramanian, 1998). This arises at a second-order FM transition due to quasielastic scattering from spin waves as $T \rightarrow T_{C}^{-}$, and from FM spin correlations as $T \rightarrow T_{C}^{+}$. The $q$ dependence can often be described by a Lorentzian:

$$
\frac{d \Sigma}{d \Omega}(q, T)=\frac{\left(d \Sigma_{L} / d \Omega\right)(T)}{q^{2}+1 / \xi(T)^{2}},
$$

where $\left(d \Sigma_{L} / d \Omega\right)$ parametrizes the strength of Lorentzian scattering, and $\xi$ is the magnetic correlation length (Furrer, Mesot, and Strässle, 2009). This $q$ dependence results from an Ornstein-Zernike spin correlation function, i.e., $\langle S(0), S(r)\rangle \propto$ $e^{-r / \xi} / r$ (Lovesey, 1984), which describes Heisenberg FMs for instance. $\xi(T)$ is thus readily obtained from SANS, Fig. 24(c) showing an example (again in $\mathrm{Tl}_{2} \mathrm{Mn}_{2} \mathrm{O}_{7}$ ) (Lynn, VasiliuDoloc, and Subramanian, 1998) of the divergence of $\xi$ as $T \rightarrow T_{C}^{+}$. SANS data as a function of $T$ in $H=0$ thus provide a detailed picture of FM ordering and were widely used to study manganites.

From around 1997, SANS was used in several studies of the evolution of FM spin correlations across $T_{C}$ in compounds such as $\mathrm{La}_{1-x} \mathrm{Ca}_{x} \mathrm{MnO}_{3}$. This was done at $x \approx 1 / 3$, where an insulator-metal transition occurs on cooling, accompanied by CMR. As shown in Fig. 24(d), De Teresa et al. found a critical-scattering-type peak at $q=0.13 \AA^{-1}$ in $\mathrm{La}_{2 / 3} \mathrm{Ca}_{1 / 3} \mathrm{MnO}_{3}$, with $T$ and $H$ dependence reminiscent of CMR (De Teresa et al., 1997). Magnetic intensity was detected from well above $T_{C}$, decreasing rapidly below it. Above $T_{C}$, Lorentzian fits [Eq. (58)] were used to extract a $\xi(T)$ exhibiting a long high $T$ tail, saturating at $12 \AA$ (i.e., 3 unit cells), and increasing to $25 \AA$ in $\mu_{0} H=5 \mathrm{~T}$. Similar data were reported for $\mathrm{Sm}_{1-x} \mathrm{Sr}_{x} \mathrm{MnO}_{3}$ (De Teresa et al., 2002). Along with resistivity, susceptibility, and thermal expansion, the SANS data on $\mathrm{La}_{2 / 3} \mathrm{Ca}_{1 / 3} \mathrm{MnO}_{3}$ were interpreted as evidence for an entity known as a magnetic polaron. Such polarons, wherein doped carriers align the spins of magnetic ions within their Bohr radii, were originally developed for magnetic semiconductors (Coey, Viret, and von Molnár, 1999; Nagaev, 2001). The conclusion of polaron formation was criticized by Viret et al., however, who claimed that $d \Sigma / d \Omega$ in a $\mathrm{La}_{0.75} \mathrm{Sr}_{0.25} \mathrm{MnO}_{3}$ single crystal was not well described by a Lorentzian or by the form factor for discrete objects such as magnetic polarons (Viret et al., 1998). Viret et al. instead advanced a correlation function of longer range than OrnsteinZernike, $\langle S(0), S(r)\rangle \propto e^{-r / \xi}, \quad$ yielding $\quad d \Sigma / d \Omega \propto \xi^{3} /$ $\left(1+\xi^{2} q^{2}\right)^{2}$. This was found to describe data above $T_{C}$ over a substantial $q$ range, yielding $\xi(T)$ similar to simpler metallic FMs. Viret et al. thus argued for typical development of magnetic coherence as $T \rightarrow T_{C}^{+}$, albeit with quantitative differences due to double exchange.

Further elucidation of magnetism near $T_{C}$ was achieved by adding energy resolution. As discussed in Sec. II.A, typical 
SANS measurements are not energy resolved, instead integrating over some window centered on energy transfer $\Delta E=0$. With an instrument such as a triple-axis spectrometer, however, energy resolution is added, and the low- $q$ intensity versus $\Delta E$ spectra in FMs probe spin waves. Performing such measurements at multiple $q$ provides the dispersion relation, from which the spin-wave stiffness $D$ can be extracted versus $T$. While somewhat different than typical INS, this approach proved expedient in early studies of manganites where it revealed an unconventional FM transition in $\mathrm{La}_{0.67} \mathrm{Ca}_{0.33} \mathrm{MnO}_{3}$. Specifically, the FM order parameter and $D(T)$ were cut off in a first-order fashion as $T \rightarrow T_{C}^{-}$, associated with the emergence of a "central peak" at $E=0$ in the low- $q$ inelastic spectrum (Lynn et al., 1996). Measurements on single crystals confirmed the first-order transition at $x=0.3$ (Adams et al., 2004). This situation was clarified by ND and INS measurements on $\mathrm{La}_{1-x} \mathrm{Ca}_{x} \mathrm{MnO}_{3}$ crystals around $x=0.3$ (Adams et al., 2000; Dai et al., 2000) (as well as layered manganites) (Vasiliu-Doloc et al., 1999; Argyriou et al., 2002), which linked the central peak to diffuse Huang scattering from nanoscale lattice polarons with shortrange correlations. These polarons, called "Jahn-Teller polarons" (due to Jahn-Teller-active $\mathrm{Mn}^{3+}$ ions), were found to generate scattering with striking similarities to the $T$ dependence of the resistivity, elucidating a subtle competition between double exchange and the lattice-charge-orbital degrees of freedom (Vasiliu-Doloc et al., 1999; Adams et al., 2000; Dai et al., 2000; Argyriou et al., 2002).

The above focused on high- $x$ manganites, probing the transition from insulating paramagnet to FMM on cooling. Further insight was obtained from studying lightly doped manganites to understand how double-exchange FMM emerges from superexchange AF at $x=0$. SANS, both conventional and when implemented with energy resolution on triple-axis systems, played a vital role here, revealing classic nanoscopic magnetic inhomogeneity. Well-studied systems include lightly hole-doped AF $\mathrm{LaMnO}_{3}$ (i.e., $\mathrm{La}_{1-x} \mathrm{Ca}_{x} \mathrm{MnO}_{3}$ ) (Hennion et al., 1998; Biotteau et al., 2001) and lightly electron-doped $\mathrm{AF} \mathrm{CaMnO}_{3}$ (i.e., $\mathrm{Ca}_{1-x} \mathrm{La}_{x} \mathrm{MnO}_{3}$ ) (Ling et al., 2003). Illustrative low- $T$ elastic magnetic SANS cross section versus $q$ plots are shown in Fig. 25 for electron- and hole-doped polycrystalline $\mathrm{Ca}_{1-x} \mathrm{La}_{x} \mathrm{MnO}_{3}$ (Ling et al., 2003). A broad peak is evident at $0.20 \AA^{-1}$, indicating nanoscale magnetic clustering in the AF matrix, with well-defined spatial extent. Single-crystal measurements (Hennion et al., 1998) showed this scattering to be isotropic, leading to the term magnetic "droplets." A quantitative analysis was performed by fitting to the general formula [see Sec. II.D, Eqs. (23)-(27)],

$$
\frac{d \Sigma}{d \Omega}(q, T)=n_{p} V_{p}^{2} \Delta \eta^{2} F^{2}(q) S(q),
$$

where $n_{p}$ and $V_{p}$ are the number density and volume of particles (in this case magnetic clusters), $\Delta \eta$ is the magnetic contrast between clusters and matrix, $F(q)$ is the cluster form factor, and $S(q)$ is the intercluster structure factor. Several group's data were well described by this (e.g., solid lines in Fig. 25), using a spherical $F(q)$, and an $S(q)$ for liquids

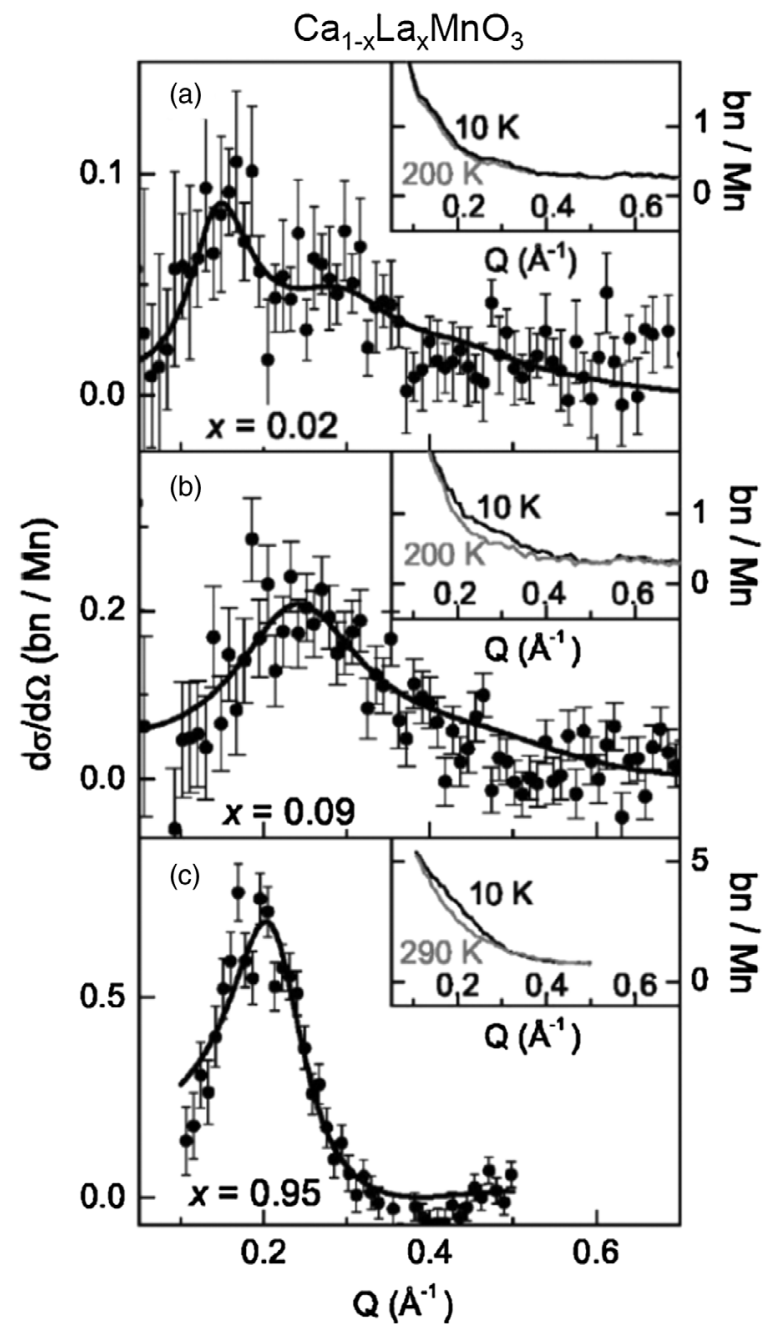

FIG. 25. $q$ dependence of the elastic magnetic SANS cross section from polycrystalline $\mathrm{Ca}_{1-x} \mathrm{La}_{x} \mathrm{MnO}_{3}$ at (a) $x=0.02$, (b) $x=0.09$, and (c) $x=0.95$. The magnetic contribution was isolated by subtracting high-temperature data, as illustrated in the insets. Solid lines are fits to a model for a liquidlike distribution of nanoscopic magnetic clusters. From Ling et al., 2003.

(Hennion et al., 1998; Biotteau et al., 2001; Ling et al., 2003). A liquidlike distribution of magnetic droplets in an AF matrix was thus concluded, with diameters $10-17 \AA$ and center-tocenter spacings $25-40 \AA$. Importantly, typical $\Delta \eta$ 's correspond to magnetization contrast of only $0.7 \mu_{B}$, indicating not simple FM clusters, but rather differing AF canting angles in the clusters and matrix (Hennion et al., 1998). Both the magnetic SANS peak intensity and cluster diameter increased with doping, low- $q$ structure and longer-range FM eventually evolving, consistent with percolation (Hennion et al., 1998; Biotteau et al., 2001; Ling et al., 2003). Notably, the charge carrier to cluster ratios extracted from $x$ and $n_{p}$ were large, $\approx 60$, favoring clusters or droplets over magnetic polarons. Several theoretical works provided support for these magnetic cluster sizes. Building on the theory discussed in Sec. VII.A, Kugel, Rakhmanov, and Sboychakov (2005) constructed a theory for manganites based on a Kondo lattice model capturing the competition between Jahn-Teller and double exchange. Electronic and/or magnetic phase separation was 
found at most dopings, and the energy was minimized to determine the FMM cluster size, accounting for long-range Coulomb interactions. The latter restrict phase separation to short length scales, $\approx 2$ unit cells, in reasonable agreement with experiment.

Nanoscale magnetic phase separation was also probed by SANS in other manganites, including $\mathrm{Ca}_{1-x} \mathrm{Bi}_{x} \mathrm{MnO}_{3}$ (Qin et al., 2008) and $\mathrm{LaMnO}_{3+\delta}$ (Ritter et al., 1997). In the latter, the substantial (positive) $\delta$ values enable a different approach to hole doping an AF manganite. FM order develops by $\delta=0.07$ according to Ritter et al., but with unusual SANS features indicating $\xi \approx 100 \AA$, even below $T_{C}$. Yet another route to explore the homogeneity of the evolution of magnetism with composition in $\mathrm{La}_{1-x} \mathrm{Ca}_{x} \mathrm{MnO}_{3}$ was realized via nonmagnetic doping on the $\mathrm{Mn}$ site, thus suppressing longrange FM. This was done in $\mathrm{La}_{2 / 3} \mathrm{Ca}_{1 / 3} \mathrm{Mn}_{1-y} \mathrm{Ga}_{y} \mathrm{O}_{3}$, SANS revealing that a transition from long-range FM order to shortrange FM correlations proceeds through an intermediate region where the two coexist (De Teresa et al., 2005). At $y \approx 0.1-0.15$, for example, long-range FM was found to coexist with short-range order with $\xi \approx 50 \AA$.

Another example of the power of SANS in elucidating magnetic inhomogeneity in manganites comes from $\operatorname{Pr}_{1-x} \mathrm{Ca}_{x} \mathrm{MnO}_{3}$ at $x \approx 0.30$. In this system the smaller $A$-site cation radius leads to weakened double exchange, typically viewed as resulting from reduced $e_{g}$-derived electronic bandwidth due to larger structural distortions (Tomioka and Tokura, 2004). Instabilities such as CO and AF thus compete with FM, a crossover occurring around $x=0.27$ (Tokura and Tomioka, 1999; Dagotto, Hotta, and Moreo, 2001; Dagotto, 2002). At $x=0.30, \operatorname{Pr}_{1-x} \mathrm{Ca}_{x} \mathrm{MnO}_{3}$ thus exhibits a $\mathrm{CO}$ transition on cooling (at $\approx 200 \mathrm{~K}$ ), followed by AF ordering (at $\approx 140 \mathrm{~K}$ ), the insulating ground state nevertheless exhibiting non-negligible magnetization (Radaelli et al., 2001). This state is remarkably sensitive to field, pressure, illumination, etc., application of a few tesla leading to a metamagnetic transition to FM [Fig. 26 (a)], with a sharp insulator-metal transition and large CMR [Fig. 26(b)] (Saurel et al., 2010). In a key step, Radaelli et al. (2001) established that the magnetization in the zero-fieldcooled, nominally charge-ordered antiferromagnet (COAF) state arises due to magnetic phase separation. COAF and FMM domains were found to coexist in polycrystals, with $T$ and $H$-dependent volume fractions, the notable feature being microscopic length scales. Very different from $\mathrm{La}_{2 / 3} \mathrm{Ca}_{1 / 3} \mathrm{MnO}_{3}$, ND revealed coexisting $\mathrm{CO}, \mathrm{AF}$, and $\mathrm{FM}$, with peak widths indicative of $>500 \AA$ length scales, and large variations in lattice parameters (Radaelli et al., 2001). This is therefore microscale phase separation, as in Fig. 23(a), as opposed to the nanoscale phenomenon in Fig. 23(b).

SANS was then applied extensively, primarily to single crystals (Yamada et al., 2001; Simon et al., 2002; Mercone et al., 2003; Yaicle et al., 2003; Saurel et al., 2006, 2007, 2010). Magnetic phase separation was established from $\approx 10 \mathrm{~nm}$ to $\approx 1 \mu \mathrm{m}$. One key observation in the $x=0.30-0.33$ range was of a crossover from the Porod form at low $q$ to a higher- $q$ regime with $d \Sigma / d \Omega \propto q^{-2}$. This was recognized as indicating reduced dimensionality, the first interpretation being rumpled 2D sheets of interpenetrating FM and AF. This "red cabbage" model is illustrated in Figs. 26(c) and 26(d); sheet thicknesses in the nm
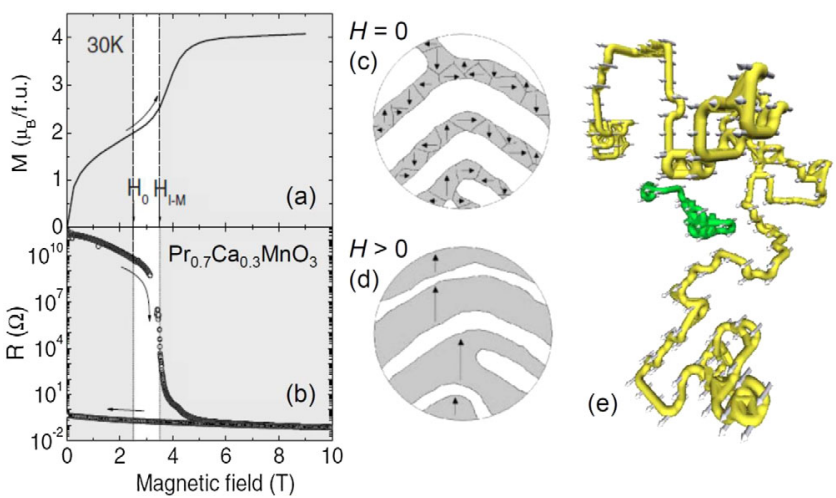

FIG. 26. (a), (b) Magnetic field dependence of the $30 \mathrm{~K}$ magnetization and resistance of a $\operatorname{Pr}_{0.7} \mathrm{Ca}_{0.3} \mathrm{MnO}_{3}$ single crystal. From Saurel et al., 2010. (c), (d) Schematic of the zero-field and in-field magnetic phase separations in the same compound. Gray signifies ferromagnetic metallic, while white is antiferromagnetic insulating. Adapted from Mercone et al., 2003. (e) Magnetic filaments obtained from Monte Carlo simulations for the same compound; arrows illustrate magnetization directions. From Viret et al., 2004.

range were reported (Simon et al., 2002; Mercone et al., 2003; Yaicle et al., 2003). In 2004, however, this picture was reevaluated by Viret et al., who detected, in a $\operatorname{Pr}_{0.67} \mathrm{Ca}_{0.33} \mathrm{MnO}_{3}$ single crystal, $q^{n}$ scattering, but with $n$ consistently 1.6 to 1.7 , i.e., $\approx 5 / 3$ (Viret et al., 2004). Taking cues from the polymer literature, this was interpreted in terms of filamentary FM chains, as in Fig. 26(e). This figure shows a Monte Carlo simulation for a "hopping exchange" process, creating a random-walk network of FM chains (Viret et al., 2004). These competing models for $\operatorname{Pr}_{1-x} \mathrm{Ca}_{x} \mathrm{MnO}_{3}$ were later compared (Saurel et al., 2006), considering Lorentzian fitting (De Teresa et al., 1997), red cabbage (Saurel et al., 2006), selfavoiding chains (Viret et al., 2004), and spherical FM regions described by a specific correlation function. While the data can be fit with multiple models, the extracted size scales were reassuringly similar.

SANS measurements have also been applied to the $H$ dependence in $\operatorname{Pr}_{1-x} \mathrm{Ca}_{x} \mathrm{MnO}_{3}$. The $\sin ^{2} \theta$ dependence in $q_{x}-q_{y}$ maps taken in finite $H$ [see Sec. II.D, Eqs. (23)-(27)] was probed in several works, and the magnetic SANS tracked versus $H$. A particularly clear view was provided by Saurel et al., who used wide $q$-range measurements to extract phase separation length scales and the specific area of FM/AF interfaces as a function of $H$ (Saurel et al., 2010). The latter can be extracted from Porod scattering [Eq. (28)]. This analysis provided a simple picture for the manner in which the FM phase fraction grows with $H$ [see Figs. 26(c) and 26(d)], as well as the resulting percolation and CMR [Fig. 26(b)], the length scales spanning from nanometric to $>1 \mu \mathrm{m}$ (Saurel et al., 2010). A special case of the $H$ dependent FM/AF phase separation, where ultrasharp $H$ and time-dependent magnetization steps were observed (Mahendiran et al., 2002), was also studied by SANS (Woodward et al., 2004). Strain effects at FM/AF boundaries were invoked to explain these avalanche phenomena.

The magnetic phase separation effects described in $\mathrm{La}_{1-x} \mathrm{Ca}_{x} \mathrm{MnO}_{3}$ (at light doping and $x \approx 1 / 3$ ) and 
$\operatorname{Pr}_{1-x} \mathrm{Ca}_{x} \mathrm{MnO}_{3}$ (at $x \approx 1 / 3$ ) are significantly different: The former, similar to $\mathrm{La}_{1-x} \mathrm{Sr}_{x} \mathrm{MnO}_{3}$, involves nanoscopic FMM clusters, with the CMR peaking at the insulator-metal transition near $T_{C}$, accompanied by only subtle structural heterogeneity (Shibata et al., 2002). In $\operatorname{Pr}_{1-x} \mathrm{Ca}_{x} \mathrm{MnO}_{3}$, however, phase separation occurs over longer scales, with clear structural differences between domains, often with sharp, percolative features in CMR. In contrast to the models discussed in Sec. VII.A, this longer-range magnetic or electronic phase separation is discussed in terms of quenched disorder near a first-order phase transition between competing phases, such as FMM and an AF insulator (Dagotto, Hotta, and Moreo, 2001; Dagotto, 2002). This occurs near $x=0.50$ in $\mathrm{La}_{1-x} \mathrm{Ca}_{x} \mathrm{MnO}_{3}$ and $x=0.30$ in $\operatorname{Pr}_{1-x} \mathrm{Ca}_{x} \mathrm{MnO}_{3}$, and is tunable in $\left(\mathrm{La}_{1-y} \mathrm{Pr}_{y}\right)_{1-x} \mathrm{Ca}_{x} \mathrm{MnO}_{3}$ (Dagotto, Hotta, and Moreo, 2001; Dagotto, 2002). The models predict emergence of clusters below some temperature $T^{*}$, with $H$-dependent competition giving CMR (Dagotto, Hotta, and Moreo, 2001; Dagotto, 2002). Models emphasizing elastic driving forces for phase separation have also been advanced (Ahn, Lookman, and Bishop, 2004). As a final comment on this issue of multiple types of magnetic phase separation, we note that two distinct forms of CMR are active in manganites, as highlighted in $\left(\mathrm{Nd}_{1-y} \mathrm{Sm}_{y}\right)_{0.5} \mathrm{Sr}_{0.5} \mathrm{MnO}_{3}$ (Tokura et al., 1996). In that work gradual $H$ dependence near the FM-to-paramagnet transition was contrasted with sharp $H$ effects at the AF/FM boundary, as discussed by Dagotto (2002).

Finally, we briefly note that SANS has also proven useful in the study of multiferroic manganites. $\mathrm{HoMnO}_{3}$ crystals, for example, exhibit AF order simultaneously with ferroelectric order below the Néel temperature of $72 \mathrm{~K}$, in addition to a spin reorientation transition at $40 \mathrm{~K}$ (Ueland et al., 2010). Strong magnetic Porod scattering was found to develop below this reorientation temperature, with sensitivity to both magnetic and electric fields. The scattering was interpreted in terms of uncompensated magnetization at $\mathrm{AF}$ domain walls, the interaction between ferroelectric and $\mathrm{AF}$ domains resulting in electric field sensitivity (Ueland et al., 2010).

\section{Perovskite cobaltites}

The discovery of CMR in the manganites stimulated interest in other perovskites, one class being cobaltites. Much like manganites, cobaltites have a long history (Jonker and Van Santen, 1953), but were studied more extensively since the 1990s. This work focused on two main issues: nanoscale magnetic phase separation and the additional spin-state degree of freedom. The latter refers to the fact that the spin state of Co ions in cobaltites can vary with composition, pressure, $H, T$, etc. This is because the crystal field splitting between $t_{2 g}$ and $e_{g}$ states is unusually close to the Hund's rule exchange energy. In insulating undoped $\mathrm{LaCoO}_{3}$, for example, the $\mathrm{Co}^{3+}$ ions adopt a low-spin $t_{2 g}^{6} e_{g}^{0}$ $(S=0)$ ground state, but with a thermally excited population of finite-spin states (simplistically the $t_{2 g}^{5} e_{g}^{1}$ intermediate spin state and $t_{2 g}^{4} e_{g}^{2}$ high-spin state) by only $30 \mathrm{~K}$; for a review see Imada, Fujimori, and Tokura (1998). An insulator-metal transition then takes place at higher $T \approx 500 \mathrm{~K}$ (Raccah and Goodenough, 1967). While much understanding of this spin-state transition or crossover has been achieved, the sequence of excited states has proven very difficult to pin down (Asai et al., 1994; Korotin et al., 1996; Noguchi et al., 2002; Haverkort et al., 2006; Podlesnyak et al., 2006; Kozlenko et al., 2007; Lee and Harmon, 2013). This is likely in part due to the desire to describe excited states in terms of low, intermediate, and high spin states, i.e., with "atomic" language (Lee and Harmon, 2013), often also ignoring spinorbit coupling (Ropka and Radwanski, 2003).

Remarkably, SANS was first applied to study the spin-state transition in $\mathrm{LaCoO}_{3}$ in 2015 (El-Khatib et al., 2015). The $T$ dependence of $d \Sigma / d \Omega$ from a $\mathrm{LaCoO}_{3}$ single crystal is reproduced in Fig. 27. At low $q$, a steep $q$ dependence is observed, with the intensity increasing on cooling, while at high $q$ the cross section is practically $q$ independent (indicating a local origin), increasing on warming. Considering the high- $q$ component first, this was shown to reflect the spin-state transition, being quasieleastic or inelastic scattering due to thermally excited paramagnetism. Specifically, earlier INS data showed thermal excitation of not only a broad inelastic paramagnetic continuum around $\Delta E=0$, but also a distinct $0.8 \mathrm{meV}$ excitation associated with the excited spin-state manifold (Phelan et al., 2006). Taking the dynamic $S(q, \omega)$ from INS and performing an energy integral appropriate for SANS confirmed quantitative consistency with the high- $q$ $\left(\approx 0.1 \AA^{-1}\right)$ SANS (El-Khatib et al., 2015), a rare example of quantification of inelastic magnetic SANS.

The low- $q$ scattering in Fig. 27, on the other hand, grows rapidly only below $60 \mathrm{~K}$, and the magnetic component was thus isolated by subtracting high- $T$ data (see inset). The result is well fit by the Guinier form [see Eq. (29) in Sec. II.D] as expected for the $q \rightarrow 0$ behavior of an assembly of scattering centers with radius of gyration $R_{g}$ (Furrer, Mesot, and Strässle, 2009; Willis and Carlile, 2009). $R_{g}$ is $140 \AA$ at low $T$, increasing with $T$ until this contribution vanishes above $60 \mathrm{~K}$. Given the unusual $R_{g}(T)$, the onset of scattering at $60 \mathrm{~K}$, and the observation of Guinier scattering from objects with

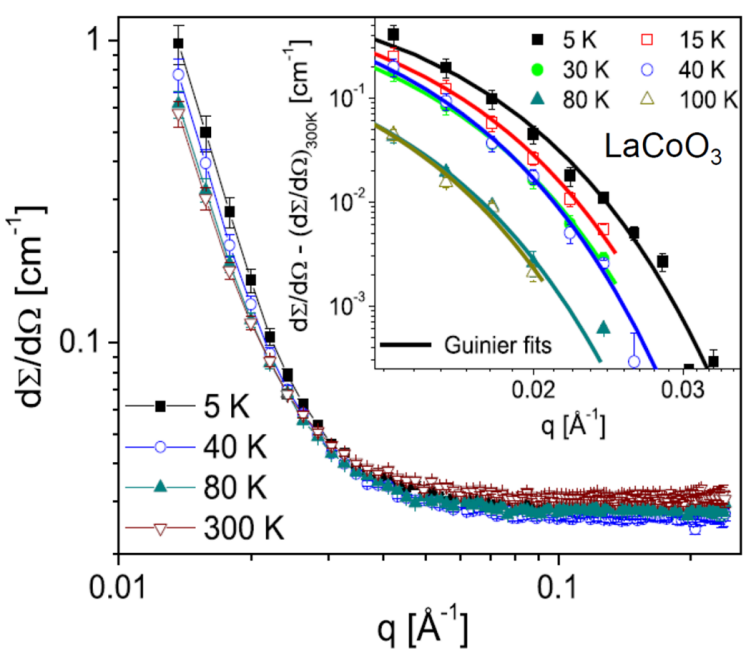

FIG. 27. $q$ dependence of the SANS cross section from single crystal $\mathrm{LaCoO}_{3}$ at multiple temperatures. Inset: Magnetic cross section, isolated by subtracting the $300 \mathrm{~K}$ data; solid lines are Guinier fits. From El-Khatib et al., 2015. 
well-defined extent, evidence for "spin excitons" or "spinstate polarons" was concluded. Such objects were theoretically proposed by Nagaev and Podel'shchikov (1996) and Podlesnyak et al. (2008), who envisioned that doped electrons or holes in $\mathrm{LaCoO}_{3}$ would stabilize finite-spin Co ions in their vicinity, aligning those spins to form a magnetic polaron in a low-spin matrix. Prior evidence for these polarons had been derived from magnetometry and muon spin spectroscopy (including collective ordering below $60 \mathrm{~K}$ ) (Giblin et al., 2005), but this was the first neutron-based detection. The scattering intensity could be explained with oxygen deficiency in $\mathrm{LaCoO}_{3-\delta}$ of only 0.0005 , the increase in $R_{g}$ on warming being a classic signature of spin polarons (El-Khatib et al., 2015).

Returning to magnetic phase separation, doped cobaltites were intensively studied from the 1990s, $\mathrm{La}_{1-x} \mathrm{Sr}_{x} \mathrm{CoO}_{3}$ emerging as a classic example of a magnetically heterogeneous oxide. Pioneering work by Goodenough had earlier established a crossover from insulating behavior to FMM around $x_{c}=0.18$, postulating formation of superparamagnetic clusters (Señarís-Rodríguez and Goodenough, 1995). A series of studies then gathered direct evidence of this (Wu and Leighton, 2003). Caciuffo, Rinaldi et al. (1999) concluded $x$-dependent phase separation into hole-rich FMM clusters in a hole-poor non-FM insulating matrix, from electron microscopy, susceptibility, ND, and thermal expansion. Kuhns et al. (2003) applied ${ }^{59} \mathrm{Co}$ zero-field and in-field NMR to polycrystalline $\mathrm{La}_{1-x} \mathrm{Sr}_{x} \mathrm{CoO}_{3}$, separately detecting the $x$-dependent FM and non-FM components. Wu et al. (2005) then applied SANS to polycrystals, coarsely spanning $x_{c}$. Low- $q$ Porod scattering from long-range FM domains was separated from high- $q$ Lorentzian scattering from nanoscale FM correlations as a function of $x$ and $T$. This solidified the picture of short-range (10-30 ̊) FM clusters at low $x$, which increase in size and density as $x$ is increased, percolating at $x_{c}$. In the same work, a large, negative, hysteretic MR was discovered in low- $x$ single crystals, arising due to spindependent intercluster transport (Wu et al., 2005), analogous to artificial systems such as $\mathrm{Co}-\mathrm{SiO}_{2}$ granular films (Sankar et al., 2000). Using INS on single crystals, Phelan et al. (2006) also detected these nanoscopic FM droplets, which were confirmed isotropic.

More detailed $\mathrm{La}_{1-x} \mathrm{Sr}_{x} \mathrm{CoO}_{3}$ SANS studies of single crystals at multiple $x$ provided a complete picture (He et al., 2009). As shown in Figs. 28(a)-28(c), T-dependent measurements provided a similar view to earlier work, the low- $q$ scattering [Fig. 28(a)] being of Porod type from FM domains, with order-parameter $T$ dependence. The higher- $q$ Lorentzian part [Fig. 28(b)] exhibits critical scattering, which weakens and broadens with decreasing $x$, resulting, below $x_{c}$, in intensity growing monotonically on cooling. The extracted $\xi(T)$ [Fig. 28(c)] evolves from divergence as $T \rightarrow T_{C}^{+}$at high $x$, to saturation (at 10-30 $\AA$ ) at low $x$. Plotting the low- $q$ $\left(0.007 \AA^{-1}\right)$ and high- $q\left(0.049 \AA^{-1}\right)$ cross sections versus $x$ at $10 \mathrm{~K}$ produces Figs. 28(d) and 28(e). Considering high $q$ first [Fig. 28(d)], the data show negligible long-range FM for $x \leq$ 0.15 as expected. This intensity then turns on around percolation (at $x_{c}=0.17-0.18$ ) peaking just above this, before leveling off above $x=0.22$. Insight into the latter
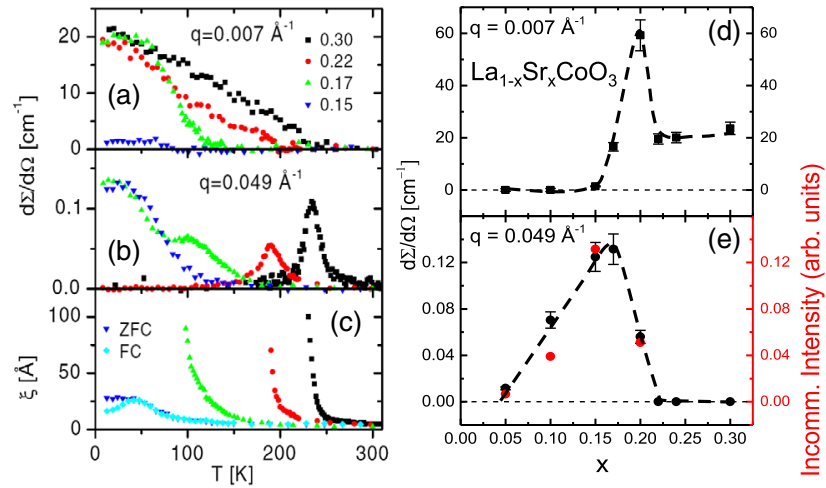

FIG. 28. Temperature dependence of the SANS cross section at (a) $q=0.007 \AA^{-1}$ and (b) $q=0.049 \AA^{-1}$ along with the extracted magnetic correlation length (c), in single crystal $\mathrm{La}_{1-x} \mathrm{Sr}_{x} \mathrm{CoO}_{3}$ at various $x$. (d), (e) The $10 \mathrm{~K} x$ dependence of the $q=0.007$ and $0.049 \AA^{-1}$ cross sections. Dashed lines are guides to the eye, and the right axis in (e) shows the normalized intensity of the incommensurate satellite peak from Phelan, Louca et al. (2006). From He et al., 2009.

observation came from the high- $q$ intensity [Fig. 28(e)], which reveals two surprises. First, this signature of short-range FM abruptly vanishes at $x=0.22$, suggesting phase-pure longrange FM above this doping. Magnetic phase separation in $\mathrm{La}_{1-x} \mathrm{Sr}_{x} \mathrm{CoO}_{3}$ crystals, unlike more disordered polycrystals (Kuhns et al., 2003), thus does not pervade the entire phase diagram, but ends abruptly at $x=0.22$, the constant low- $q$ scattering above this coming from conventional FM domains. The second surprise from the low- $q$ SANS is that the scattering, which peaks near percolation, also vanishes at low $x$, around 0.04 . This observation of a finite window for magnetic phase separation in the highest quality $\mathrm{La}_{1-x} \mathrm{Sr}_{x} \mathrm{CoO}_{3}$ was corroborated by multiple techniques, including La NMR and heat capacity, each in precise agreement on the lower and upper doping limits of 0.04 and 0.22 (Smith et al., 2008; He et al., 2009).

These observations were used by He et al. (2009) as the starting point for statistical modeling showing that on the scales of the observed $\xi$ values [Fig. 28(c)], local compositional fluctuations are sufficient to explain the experiments. With no adjustable parameters, simulations were shown to predict start and end values for the phase-separated regime of 0.04 and 0.21 , in remarkable agreement with experiment. The model also reproduces key features of the $x$-dependent SANS cross sections, as well as volume fractions of FMM and nonFMM phases. This demonstrated that nanoscale magnetic inhomogeneity in $\mathrm{La}_{1-x} \mathrm{Sr}_{x} \mathrm{CoO}_{3}$ can be explained solely via local compositional fluctuations that must always be present in randomly doped solids on such short scales, with no need to invoke electronic mechanisms. Understanding to what extent similar local compositional and structural inhomogeneities occur in other systems with nanoscale magnetic inhomogeneity remains an open challenge.

One unresolved issue in $\mathrm{La}_{1-x} \mathrm{Sr}_{x} \mathrm{CoO}_{3}$ arose from the work of Phelan, Louca et al. (2006), who detected unanticipated incommensurate scattering around the (001) FM Bragg peak. The deduced spin superstructure was found to have $x$ - and 
$T$-dependent intensity and incommensurability, speculatively linked to spin-state polarons. As discussed, these spin-state polarons form around doped carriers in $\mathrm{LaCoO}_{3-\delta}$. In the case of $\mathrm{La}_{1-x} \mathrm{Sr}_{x} \mathrm{CoO}_{3}$ they were shown to be octahedrally coordinated complexes from the $q$ dependence of a characteristic 0.75 meV excitation (Podlesnyak et al., 2008). While the exact link between these polarons and incommensurate magnetism remains unclear, Fig. 28(e) provides some insight by overlaying the incommensurate intensity (Phelan, Louca et al., 2006) with the high- $q$ SANS cross section (He et al., 2009). The comparison is striking, indicating that the two forms of scattering probe the same physics. Another open issue in $\mathrm{La}_{1-x} \mathrm{Sr}_{x} \mathrm{CoO}_{3}$ is the relationship between the spin-state polarons at light doping, and the magnetic clusters at higher $x$. While much remains to be learned about the distinguishing features of these entities, as well as how one evolves into the other, recent work took a first step. Specifically, INS and magnetometry data were acquired versus $x$, indicating "collapse" of the spin-state polarons into magnetic clusters, evidenced by a rapid drop in magnetization per doped hole (Podlesnyak et al., 2011).

We note as an aside here that magnetic phase separation in $\mathrm{La}_{1-x} \mathrm{Sr}_{x} \mathrm{CoO}_{3}$ also plays a role in heterostructures. A central problem in such systems is the suppression of the FMM phase in ultrathin films. This "dead layer" effect has been extensively studied in $\mathrm{La}_{0.7} \mathrm{Sr}_{0.3} \mathrm{MnO}_{3}$ and $\mathrm{La}_{0.5} \mathrm{Sr}_{0.5} \mathrm{CoO}_{3}$ films, including in the latter case with SANS. The crossover from FMM to non-FM insulating phases below $70 \AA$ thickness in $\mathrm{SrTiO}_{3}(001) / \mathrm{La}_{0.5} \mathrm{Sr}_{0.5} \mathrm{CoO}_{3-\delta}$, for example, was shown to be driven by a decrease in effective doping, due to accumulation of interfacial oxygen vacancies (Torija et al., 2011). In a novel mechanism, these vacancies play the key role in strain relaxation, ordering into alternating oxygen deficient or sufficient planes, similar to brownmillerite $\mathrm{Sr}_{2} \mathrm{Co}_{2} \mathrm{O}_{5}$ $\left(\mathrm{SrCoO}_{2.5}\right)$, to better lattice match the substrate (Gazquez et al., 2013). TEM with electron energy loss spectroscopy showed that the effective doping thereby falls to $\approx 0.22$ at the interface with $\mathrm{SrTiO}_{3}(001)$, triggering magnetic phase separation. Exactly this was detected by SANS in $650 \AA$-thick films. Scattering of the form $q^{n}$ was observed at low $q$, with $n=2.7$ and order-parameter shape (Torija et al., 2011). This derives from long-range FM domains, the low $n$ indicating domain-wall pinning at defects. At higher $q$, a weaker $q$ dependence was found, the $T$ dependence revealing critical scattering, as expected, but on a background of additional intensity not present in bulk. This short-range FM was interpreted as arising from the interface, directly evidencing interface-induced magnetic phase separation as the origin of the dead layer in $\mathrm{La}_{1-x} \mathrm{Sr}_{x} \mathrm{CoO}_{3}$ films (Torija et al., 2011). This represents one of the few applications of SANS to thin film oxides. While intensity limited (multiple films were stacked), this work demonstrates the feasibility of SANS on complex oxide heterostructures, which could expand in the future.

The majority of the discussion of $\mathrm{La}_{1-x} \mathrm{Sr}_{x} \mathrm{CoO}_{3}$ focused on the evolution in magnetism as $x$ is varied at low $T$. A complementary approach is to understand how the FMM state evolves with $T$, as first studied with SANS by Caciuffo, Mira et al. (1999). In that work, the behavior around $T_{C}$ in polycrystalline $\mathrm{La}_{0.7} \mathrm{Sr}_{0.3} \mathrm{CoO}_{3}$ revealed $T$-dependent magnetic inhomogeneity. The SANS included a strongly $T$ dependent Guinier component, indicating an asymmetric peak in magnetic intensity and cluster size (peaking at $15 \AA$ ) around $T_{C}$. This was interpreted as preformation of magnetic clusters that are eventually subsumed by longer-range FM at low $T$. A somewhat similar picture was concluded from SANS on polycrystalline $\mathrm{La}_{1-x} \mathrm{Sr}_{x} \mathrm{CoO}_{3}$ at $x=0.20-0.50$ (He et al., 2007). As shown in Figs. 29(a)-29(c), that work found critical scattering, well described by Eq. (58). Interestingly, the deduced $\xi(T)$ exhibits well-defined onset temperatures for FM spin correlations [Figs. 29(d) and 29(f) and insets], unlike the typical power-law growth as $T \rightarrow T_{C}^{+}$. The susceptibility also exhibits deviations from Curie-Weiss behavior at this temperature, $T^{*} \approx 360 \mathrm{~K}$. These findings are reminiscent of the Griffiths model, referring to a classic theoretical treatment of randomly diluted Ising FMs (Griffiths, 1969; Bray, 1987). In that model, cooling below the undiluted $T_{C}$ results in a spatially inhomogeneous clustered phase, with nonanalytical
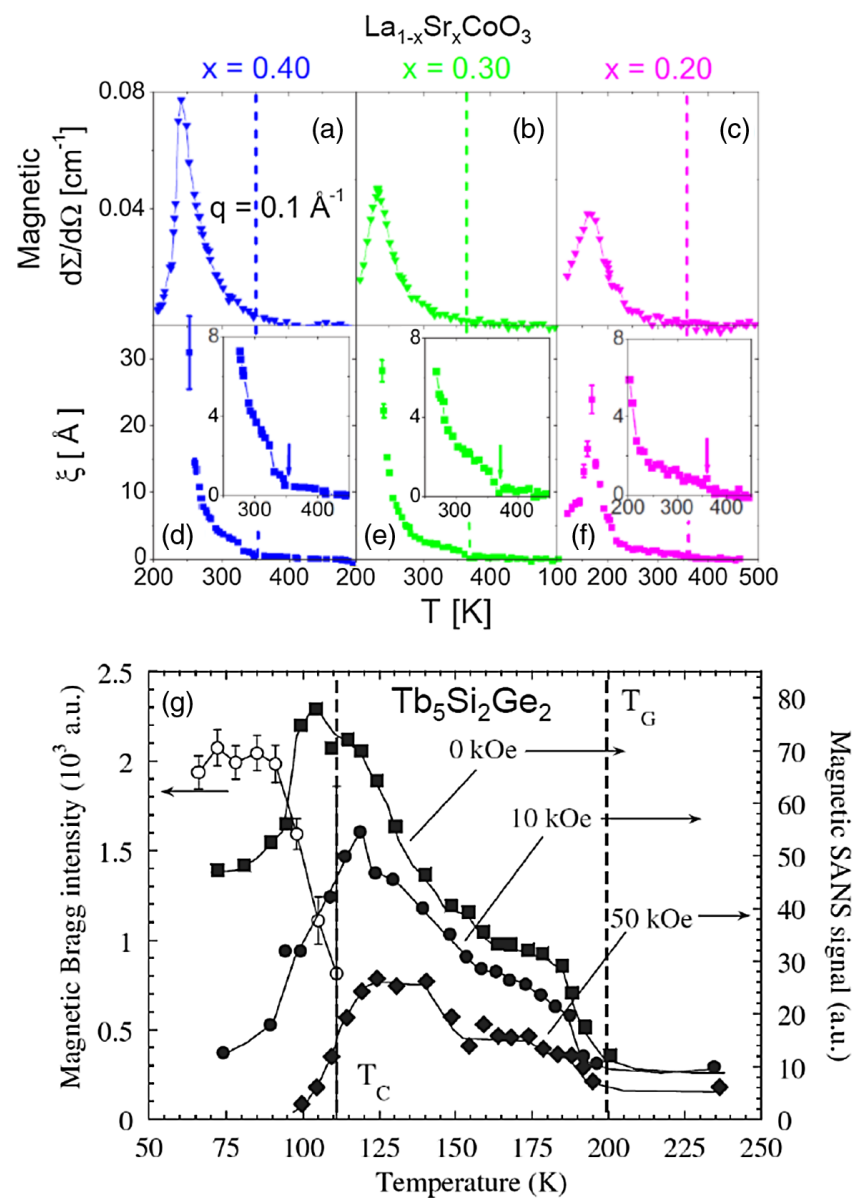

FIG. 29. Temperature dependence of the $q=0.1 \AA^{-1}$ SANS cross section (a)-(c) and magnetic correlation length (d)-(f) of polycrystalline $\mathrm{La}_{1-x} \mathrm{Sr}_{x} \mathrm{CoO}_{3}$ at $x=0.40,0.30$, and 0.20 . The insets in (d)-(f) are expanded views of the low correlation length region, the arrows marking $T^{*}$, where correlations turn on. From He et al., 2007. (g) Temperature dependence of the ferromagnetic Bragg intensity (left axis), and $q=0.1 \AA^{-1}$ magnetic SANS intensity (right axis) of polycrystalline $\mathrm{Tb}_{5} \mathrm{Si}_{2} \mathrm{Ge}_{2}$ at various magnetic fields. Curie and Griffiths temperatures are marked with vertical dashed lines. From Magen et al., 2006). 
behavior of thermodynamic quantities. The applicability of this model to manganites was quite intensively studied, with several authors concluding preformation of magnetic clusters in a manner consistent with a Griffiths phase (Salamon, Lin, and Chun, 2002; Deisenhofer et al., 2005). In $\mathrm{La}_{1-x} \mathrm{Sr}_{x} \mathrm{CoO}_{3}$, however, the specifics were found quite different from Griffiths, including the sign of the deviations from CurieWeiss behavior (He et al., 2007). A well-defined onset temperature for FM clustering nevertheless occurs, and it remains to be clarified why various perovskite oxides behave differently with regard to comparisons to the Griffiths model.

Finally, we briefly mention that SANS has also been used as a probe of nanoscale magnetic order in other cobaltites. $\mathrm{Pr}_{1-x} \mathrm{Sr}_{x} \mathrm{CoO}_{3}$ is one example, primarily because compositions around $\operatorname{Pr}_{0.5} \mathrm{Sr}_{0.5} \mathrm{CoO}_{3}$ display a second magnetic transition below $T_{C}$ (Mahendiran and Schiffer, 2003). This was eventually shown, with the assistance of SANS, to signify a change in magnetocrystalline anisotropy, driven by an unusual isomorphic structural transition (Leighton et al., 2009). Further decreasing the average cation radius leads to $\mathrm{Pr}_{1-x} \mathrm{Ca}_{x} \mathrm{CoO}_{3}$. In this material the suppression of the FMM phase leads to acute competition with other instabilities, inducing complex phase separation. Based in large part on SANS, polycrystalline $\operatorname{Pr}_{0.7} \mathrm{Ca}_{0.3} \mathrm{CoO}_{3}$ was shown to separate into short-range- and long-range-ordered FM phases, with different coercivities (El-Khatib et al., 2010). The coupling between the two was then found to create a hard or soft FM composite displaying exchange spring behavior. At higher $x$, $\operatorname{Pr}_{1-x} \mathrm{Ca}_{x} \mathrm{CoO}_{3}$ was discovered to exhibit an extraordinary first-order metal-insulator transition driven by a $\mathrm{Pr}$ valence transition. This effect was first detected in $\operatorname{Pr}_{0.5} \mathrm{Ca}_{0.5} \mathrm{CoO}_{3}$ (Tsubouchi et al., 2002) and later stabilized via Y substitution (Hejtmanek et al., 2010). SANS has again been applied to these systems, not only to probe the FM order in related compounds (e.g., $\mathrm{Nd}_{1-x} \mathrm{Ca}_{x} \mathrm{CoO}_{3}$ ) (Phelan et al., 2013), but also to understand the evolution of magnetism across the valence transition (Phelan et al., 2014). The latter measurements reveal striking inhomogeneity, complementary to chemical and structural studies by TEM (Gulec et al., 2016). These valence transitions remain an active research area in cobaltites.

\section{Complex magnetic alloys}

As illustrated in other sections of this review (e.g., Sec. III on Nd-Fe-B and Sec. IV on steels), SANS has a long history of applicability to magnetic alloy systems, in addition to oxides. Along with the study of micromagnetics in alloys, phenomena such as spin-glass freezing have been extensively studied with SANS. In terms of nanoscale magnetic inhomogeneity, alloys exhibiting reentrant spin-glass behavior are of particular interest. In these materials, such as $\mathrm{Pd}-\mathrm{Fe}-\mathrm{Mn}, \mathrm{Cr}-\mathrm{Fe}, \mathrm{Au}-$ $\mathrm{Fe}, \mathrm{Ni}-\mathrm{Mn}, \mathrm{Fe}_{3-x} \mathrm{Al}_{x}, a-\left(\mathrm{Fe}_{1-x} \mathrm{Mn}_{x}\right)_{75} \mathrm{P}_{16} \mathrm{~B}_{6} \mathrm{Al}_{3}$, and $a-\mathrm{Fe}-\mathrm{Zr}$, a paramagnet-to-FM transition on cooling is followed by a transition to a spin glass, i.e., from an ordered to a glassy state (Shapiro et al., 1980; Aeppli et al., 1983; Rhyne and Fish, 1985; Garcia-Calderón et al., 2005). SANS has been used for decades to probe magnetic ordering and correlation lengths in such alloys (Mettus et al., 2017), which have proven challenging to understand. As discussed in $a-\mathrm{Fe}_{1-x} \mathrm{Zr}_{x}$, nanoscale magnetic inhomogeneity is common to essentially all models, where FM order coexists with spin-glass regions (GarciaCalderón et al., 2005). An important advance was made in 1983 , when SANS studies on $a-\left(\mathrm{Fe}_{1-x} \mathrm{Mn}_{x}\right)_{75} \mathrm{P}_{16} \mathrm{~B}_{6} \mathrm{Al}_{3}$ were used to develop a heuristic model for reentrant spin glasses, based on random-field effects in systems with coupled FM and spin-glass order parameters (Aeppli et al., 1983). The model emphasizes coupling between spin-glass and FM networks, introducing a scattering function based on a sum of Lorentzian and Lorentzian-squared terms:

$$
\frac{d \Sigma}{d \Omega}(q, T)=\frac{A(T)}{q^{2}+\xi_{A}^{-2}(T)}+\frac{B(T)}{\left[q^{2}+\xi_{B}^{-2}(T)\right]^{2}},
$$

where $A$ and $\xi_{A}$ are defined as in Eq. (58), and $B$ and $\xi_{B}$ result from the random field, which creates finite FM regions. This function has been widely employed, although its general applicability is not entirely clear (Garcia-Calderón et al., 2005). The similarity of Eq. (60) to a power law $\left(d \Sigma / d \Omega \propto q^{-n}\right)$ in the experimental $q$ range likely complicates this situation (Aeppli et al., 1983). While there remain competing models in materials such as $\mathrm{Fe}_{1-x} \mathrm{Zr}_{x}$, involving finite AF and FM clusters, long-range FM networks, random anisotropy fields, noncollinear spin structures, and separately responding longitudinal and transverse spins, nanoscopic magnetic inhomogeneity features prominently (GarciaCalderón et al., 2005).

Other examples of SANS elucidation of magnetic inhomogeneity in complex alloys arise in magnetocaloric intermetallics and transition metal and rare-earth alloys. One example of the former is highlighted in Fig. 29(g), where the $T$ dependence of the FM Bragg intensity (left axis) and $q=0.1 \AA^{-1}$ SANS (right axis) are plotted for $\mathrm{Tb}_{5} \mathrm{Si}_{2} \mathrm{Ge}_{2}$ (Magen et al., 2006). This compound is a member of the series $\mathrm{RE}_{5}\left(\mathrm{Si}_{1-x} \mathrm{Ge}_{x}\right)_{4}(\mathrm{RE}=$ rare earth$)$, in which giant magnetocaloric effects occur. Interestingly, as shown in Fig. 29(g), in $\mathrm{Tb}_{5} \mathrm{Si}_{2} \mathrm{Ge}_{2}$ the FM ordering at $T_{C}=110 \mathrm{~K}$ is preceded by the onset of magnetic SANS at $200 \mathrm{~K}$. In the interval $110<T<200 \mathrm{~K}$, the behavior of the susceptibility $d \Sigma / d \Omega(q, T)$ and $\xi(T, H)$ (from Lorentzian fitting) were shown consistent with the Griffiths model (see Sec. VII.B.2). The $200 \mathrm{~K}$ temperature scale was identified with the $T_{C}$ of the Si-rich $\mathrm{Tb}_{5}\left(\mathrm{Si}_{x} \mathrm{Ge}_{1-x}\right)_{4}$ end member, leading to an interpretation where the disorder results from structural or chemical fluctuations (Magen et al., 2006). An example of the use of SANS to elucidate magnetic inhomogeneity in correlated electron systems is found in $\mathrm{CeNi}_{1-x} \mathrm{Cu}_{x}$, where percolation from a cluster glass to long-range FM order was proposed (Marcano et al., 2007).

The remainder of this section focuses on a different class of complex alloys that have recently been shown to display magnetic phase separation. We refer here to off-stoichiometric Heusler alloys. Full Heusler and half Heusler alloys are simply ordered cubic alloys of the form $X_{2} Y Z$ and $X Y Z$ (Felser and Hirohata, 2015). Many such alloys are magnetic, including numerous FMs, such as $\mathrm{Ni}_{2} \mathrm{MnSn}$. The latter has $T_{C}=340 \mathrm{~K}$, the moment residing primarily on $\mathrm{Mn}$ (Krenke et al., 2005). One of the many contemporary research areas with these Heuslers involves deliberate generation of 
nonstoichiometry by substituting excess $\mathrm{Mn}$ for $\mathrm{Sn}$, resulting in disordered alloys such as $\mathrm{Ni}_{50} \mathrm{Mn}_{25+y} \mathrm{Sn}_{25-y}$ (Krenke et al., 2005). The excess Mn creates Mn-Mn bonds, with strongly $\mathrm{AF}$ character, thus generating tunable $\mathrm{FM}$ and $\mathrm{AF}$ phase competition. These off-stoichiometric Heuslers often also exhibit first-order martensitic phase transformations from cubic to lower symmetry on cooling, with substantial impact on magnetism (Krenke et al., 2005; Bhatti et al., 2012, 2016). This results in ferroelasticity, multiferroicity, shape memory behavior, magnetocaloric effects, etc., with potential applications in sensors, actuators, refrigeration, and energy conversion (Krenke et al., 2005; Bhatti et al., 2012, 2016).

A central issue in such alloys is the magnitude of the thermal hysteresis at the martensitic transformation. Recent theory has had a dramatic impact in this respect, identifying geometrical compatibility criteria between the austenite and martensite phases that minimize hysteresis (Cui et al., 2006; Zhang, James, and Müller, 2009). Complex alloys such as $\mathrm{Ni}_{50-x} \mathrm{Co}_{x} \mathrm{Mn}_{25+y} \mathrm{Sn}_{25-y}$ (Bhatti et al., 2012, 2016) and $\mathrm{Ni}_{50-x} \mathrm{Co}_{x} \mathrm{Mn}_{25+y} \mathrm{In}_{25-y}$ (Karaca et al., 2009) thus emerged, possessing close-to-300 $\mathrm{K}$ phase transformations, with sub$10 \mathrm{~K}$ hysteresis. The phase diagram of $\mathrm{Ni}_{50-x} \mathrm{Co}_{x} \mathrm{Mn}_{40} \mathrm{Sn}_{10}$ is shown in Fig. 30(a) (Bhatti et al., 2016). The martensitic phase transformation temperature decreases gradually from 430 to $310 \mathrm{~K}$ from $x=0$ to 10 , before dropping by over $300 \mathrm{~K}$ with $1 \%$ additional Co. For $x>4$, FM emerges in the austensite, the martensitic transformation inducing a firstorder transition from $\mathrm{FM}$ to non-FM on cooling. Compositions around $\mathrm{Ni}_{44} \mathrm{Co}_{6} \mathrm{Mn}_{40} \mathrm{Sn}_{10}$ thus exhibit a soft FM austenite phase with high $T_{C}(430 \mathrm{~K})$, transforming at just above ambient to non-FM martensite with $\approx 1000 \mathrm{emu} / \mathrm{cm}^{3}$ magnetization change across only a $6 \mathrm{~K}$ hysteresis region (Bhatti et al., 2016).

Detailed magnetic studies of this and related alloys reveal a number of unanticipated low- $T$ features, including substantial magnetization in the nominally non-FM state, Langevin-like $T$ and $H$ dependences, magnetic freezing transitions, and "intrinsic" exchange bias (Cong et al., 2010; Wang et al., 2011; Cong, Roth, and Schultz, 2012). The superparamagnetlike behavior led to various hypotheses of magnetic clustering, recently verified by SANS (Bhatti et al., 2012). Specifically, a study of $\mathrm{Ni}_{44} \mathrm{Co}_{6} \mathrm{Mn}_{40} \mathrm{Sn}_{10}$ revealed not only the typical low- $q$ Porod and high- $q$ Lorentzian components associated with long-range FM domains and short-range FM spin correlations, but also a distinct peak at intermediate $q$. A $T$-dependent summary is provided in Figs. 30(b) and 30(c), where $T_{C}$ is marked by the onset of FM domain scattering at $q=$ $0.005 \AA^{-1}$ [Fig. 30(b)], as well as a critical scattering peak at $q=0.1 \AA^{-1}$ [Fig. 30(c)]. The weakly hysteretic FM to nonFM transition at the martensitic transformation is also clear below $400 \mathrm{~K}$, but with non-negligible high- $q$ scattering in the low- $T$, nominally non-FM phase. As shown in the inset, this weak scattering reveals a clear phase transition, associated with the intermediate $q$ peak. The peak was interpreted as an $S(q)$-derived feature reflecting a liquidlike distribution of FM clusters with center-to-center spacing of $12 \mathrm{~nm}$. Estimates of cluster diameters from magnetization data result in $\approx 2 \mathrm{~nm}$, providing a consistent interpretation, and the first direct confirmation of nanoscale magnetic clusters (Bhatti et al.,
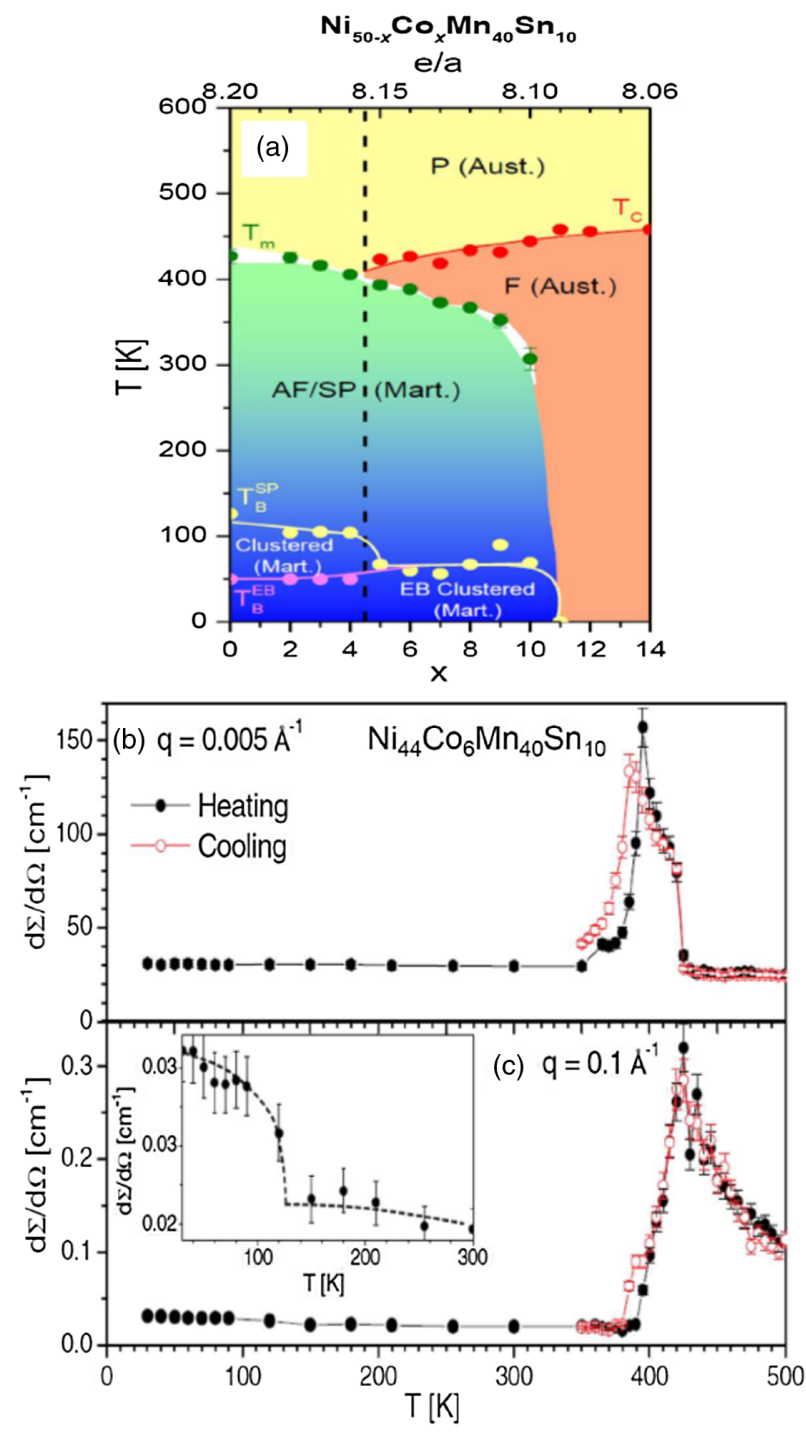

FIG. 30. (a) Phase diagram of $\mathrm{Ni}_{50-x} \mathrm{Co}_{x} \mathrm{Mn}_{40} \mathrm{Sn}_{10}$. From Bhatti et al., 2016. $T_{M}, T_{C}, T_{B}^{S P}$, and $T_{B}^{E B}$ denote the martensitic phase transformation temperature, Curie temperature, and superparamagnetic and exchange bias blocking temperatures, respectively. P, F, AF, and SP denote paramagnetic, ferromagnetic, antiferromagnetic, and superparamagnetic; "Aust." and "Mart." refer to austenite and martensite. The top axis shows the valence electron per atom ratio. (b), (c) The temperature dependence of the SANS cross section from $\mathrm{Ni}_{44} \mathrm{Co}_{6} \mathrm{Mn}_{40} \mathrm{Sn}_{10}$ at $q=0.005$ and $0.1 \AA^{-1}$, taken on cooling and heating. The inset shows a close-up at low temperature. From Bhatti et al., 2012.

2012). SANS peak widths further indicated correlation lengths > $600 \AA$, evidencing strong intercluster interactions (Cong et al., 2010; Wang et al., 2011). It should be noted here that the blocking temperature in SANS [Fig. 30(c), inset] is significantly larger than in magnetometry, due to the short time scales probed. The latter issue also arose in ${ }^{55} \mathrm{Mn}$ NMR measurements of the same alloys (Yuan et al., 2015, 2016). These yielded considerable insight, including a complex interplay between the nanoscale FM clusters and short-range AF order in the martensitic matrix (Bhatti et al., 2016). Further work will be required to understand the magnetism in these multicomponent alloys and to test ideas based on 
compositional fluctuations (Bhatti et al., 2012, 2016). SANS appears well suited to these efforts.

\section{SKYRMION LATTICES AND NONCOLLINEAR SPIN STRUCTURES}

The recent discovery of a magnetic skyrmion lattice (SkL) in $\mathrm{MnSi}$ (Mühlbauer, Binz et al., 2009) provided a showcase for a new type of magnetic order, where magnetic whirls exhibit particlelike properties due to their nontrivial topology. With SANS in a leading role, magnetic skyrmions are observed in increasing number in a wide range of material classes rendering their emergence as generic phenomenon of materials which promote noncollinar chiral magnetic interactions. Together with the ease of manipulating SkLs with ultralow current densities, their topological (and sometimes multiferroic) properties ideally suit future data storage and logical devices.

This section first provides an introduction into the variety of competing or oscillatory interactions (cf. Sec. VIII.A.1) including the DMI (cf. Sec. VIII.A.2) that lead to the large zoo of noncollinar spin structures. We then review the intrinsic multiferroic properties of noncollinear spin structures (cf. Sec. VIII.A.3) before we concentrate on their properties accessible by means of SANS (cf. Sec. VIII.A.5) and the peculiarities of diffraction in SANS geometry (cf. Sec. VIII.A.4). We finally summarize recent developments in the area of magnetic skyrmions (cf. Sec. VIII.B.1 and VIII.B.2).

\section{A. Noncollinear magnetic structures}

Spiral or noncollinear spin structures describe a large variety of spin modulations which share a rotation of the magnetic moment or spin, whether localized or itinerant, from one unit cell to the next by canting angle $\alpha_{r}$. Such modulations can be described by a single propagation vector $\mathbf{k}$, such as, e.g., in helical, cycloidal, longitudinal-conical or transverseconical spin spirals. The superposition or mixture of multiple propagation vectors, even with different moduli of $\mathbf{k}$, leads to more complex textures such as, e.g., helifan or skyrmionic structures with potentially nontrivial topology. Note that the different propagation vectors of multi-k structures exhibit a fixed phase relation with respect to each other while multidomain single-k structures have no fixed phase relation. Noncollinar spin structures exhibit a wide range of wavelengths from a few atomic layers to smooth rotations on a scale of several thousand $\AA$.

\section{Frustrated and oscillating interactions}

A large variety of noncollinear spin structures can be caused by frustrated and oscillating interactions. Here we provide some typical examples illustrating the different coupling mechanisms. For further details see Chatterji (2006) and Coey (1987), Kawamura (1998), and Freeman and Nakamura (2004).

The interplay of FM or AF nearest-neighbor interactions and AF next-nearest-neighbor coupling can lead to a spin spiral ground state (Yoshimori, 1959a, 1959b; Enz, 1961; Nagamiya, Nagata, and Kitano, 1962). A typical example for a Mott insulator showing spiral spin structures is perovskite $\mathrm{TbMnO}_{3}$ (Kimura et al., 2003). While this mechanism is relevant for localized and itinerant electron materials, longrange interactions of the electron gas are expected to play an additional role for the latter. For itinerant systems, the role of these interactions is reflected in the structure of the electronic response. In the case the spin susceptibility exhibits a maximum at a finite wave vector $\mathbf{k}$, this may result in the formation of a spin density wave (SDW) or spin spiral with propagation vector $\mathbf{k}$, respectively. Moreover, a real-space oscillatory RKKY interaction (Ruderman and Kittel, 1954; Kasuya, 1956; Yosida, 1957) can be induced by a kink or a derivative singularity (for a $2 \mathrm{D}$ or $3 \mathrm{D}$ electron gas, respectively), leading to the formation of noncollinear spin structures. Examples include the good metal FeAs (Selte, Kjekshus, and Andresen, 1972; Segawa and Ando, 2009) and the rare-earth metal Ho, where RKKY exchange between the localized $4 f$ spins through the conduction electrons leads to a variety of spiral phases (Coqblin, 1977; Szary et al., 2016). In the strongly correlated $f$ electron material $\mathrm{CeRhIn}_{5}$ the RKKY spiral state is strongly coupled to the conduction electrons via the Kondo effect (Fobes et al., 2018) generating emergent electronic heterostructures. Here, the spin fluctuations related to the spiral state are believed to be closely tied to the formation of textured superconductivity and electronic nematic textures (Ronning et al., 2017).

Noncollinear spin structures can also be caused by geometric frustration as, e.g., in the spinel Chromites $\mathrm{ZnCr}_{2} \mathrm{Se}_{4}$ and $\mathrm{CdCr}_{2} \mathrm{O}_{4}$ (Plumier, 1966; Siratori et al., 1980; Chung et al., 2005; Murakawa et al., 2008). Recently, Azhar and Mostovoy (2017) pointed out that the FM state of systems showing double exchange is generally unstable against the formation of noncollinear spin structures. Typical examples are the cubic perovskite compound $\mathrm{SrFeO}_{3}$ (Ishiwata et al., 2011) and $\mathrm{MnAu}_{2}$, actually one of the oldest spin spiral materials known (Meyer and Taglang, 1956; Herpin and Meriel, 1961). It was argued that the spiral state is induced by a competition of short-range $\mathrm{AF}$ and long-range interactions induced by the polarization of the $\mathrm{Au}$ bands (Glasbrenner, Bussmann, and Mazin, 2014).

In general, a large variety of spiral wavelengths are found for frustrated and oscillatory interactions (Glasbrenner, Bussmann, and Mazin, 2014). Furthermore, if chirality is present, the samples show a mixture of left and right chiral domains.

\section{Dzyaloshinskii-Moriya interaction}

For crystalline space groups lacking inversion symmetry, the largest term of the anisotropic superexchange that is linear in the spin-orbit coupling has the antisymmetric form $H_{\mathrm{DM}}=$ D $\cdot\left(\mathbf{S}_{i} \times \mathbf{S}_{j}\right)$ denoted as DMI (Dzyaloshinskii, 1958; Moriya, 1960). The DMI favors a perpendicular rather than a FM or AF arrangement of spins. $\mathbf{D}$ is a vector, which depends on the symmetry of the magnetic exchange path of the two involved spins. In contrast to frustrated and oscillatory interactions, magnetic structures involving the DMI can be conveniently seen as the result of a hierarchy of energy scales with symmetric exchange (FM or AF) $J_{1}$ as the leading contribution. Because of weaker spin-orbit coupling, the DMI term leads to a small canting of the FM or AF parent structure, the local arrangement of spins is hence almost FM or AF, as schematically indicated in Fig. 31(a). The associated 
(a)
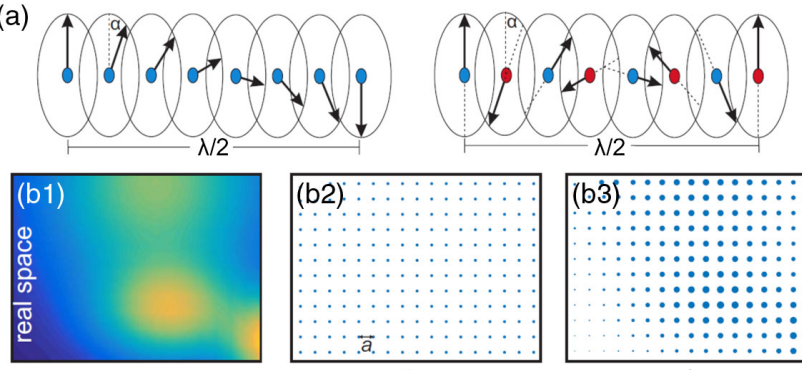

$(\mathrm{b} 2)$

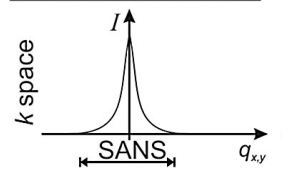

(c)

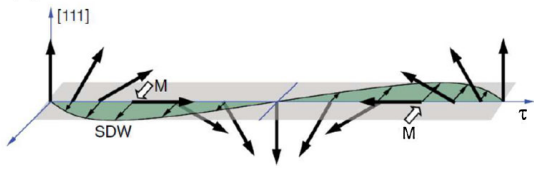

(d) 0.
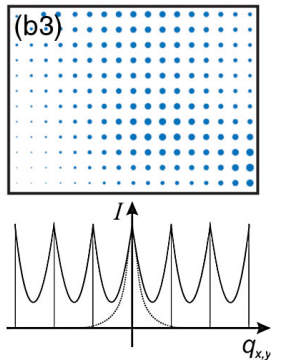

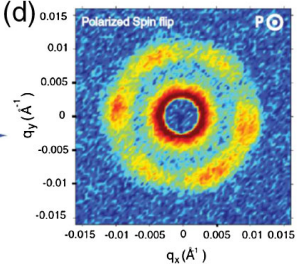

FIG. 31. (a) Schematic depiction of a spin helix derived from a FM (left) or AF (right) spin structure. (b1)-(b3) The transformation from real space to reciprocal space for a smooth texture (b1), a discrete atomic lattice with lattice spacing $a$ (b2), and a smooth incommensurate modulation on top of a discrete atomic lattice (b3) where the width of the points corresponds to the color code of (b1). (c) SDW of multiferroic $\mathrm{BiFeO}_{3}$ (thin arrows) that is caused by a slight canting of an otherwise AF cycloidal spin spiral (thick arrows show the staggered moment of the AF cycloidal structure). The canting leads to a small FM SDW visible by polarized SANS (d). (c), (d) From Ramazanoglu et al., 2011.

propagation vector $\mathbf{k}$ is given by $D / J_{1}$, leading to modulations that are long compared to typical interatomic distances, $100 \AA$ to a few $1000 \AA$. Note that weak FM behavior induced by canting of spins due to DMI should not be confused with weak itinerant FM, which is characteristic of a small localized but a large fluctuating moment (Lonzarich and Taillefer, 1985).

The spin structure (e.g., helix or cycloid) is fixed by the interplay of magnetocrystalline anisotropies and the sign and orientation of $\mathbf{D}$, hence the crystallographic space group. Zeeman coupling to the external magnetic field can lead to a wealth of different spiral structures and associated phase transitions, respectively. As the orientation and sign of $\mathbf{D}$ is solely derived from the noncentrosymmetric crystallographic space group, chiral magnetic structures based on DMI show a single chirality or handedness (Grigoriev et al., 2014) rather than statistical mixtures of left- and right-handed structures. However, a noncentrosymmetric crystallographic space group not necessarily implies a chiral magnetic structure. Note that also surfaces and interfaces locally break inversion symmetry and hence allow for DMI terms.

A large body of work exists on the wealth of DMI helimagnets. To name a few prominent examples, $\mathrm{Cr}_{1 / 3} \mathrm{NbS}_{2}$ (Togawa et al., 2012), $\mathrm{NdFe}_{3}\left(\mathrm{BO}_{3}\right)_{4}$ (Janoschek et al., 2010), and $\mathrm{CuB}_{2} \mathrm{O}_{4}$ (Roessli et al., 2001) show helimagnetic order which distorts to a soliton lattice.

$\mathrm{CsCuCl}_{3}$ (Adachi, Achiwa, and Mekata, 1980) is a further classic helimagnetic material that exhibits a DMI based spin helix. Noncentrosymmetric $\mathrm{MnSi}\left(P 2_{1} 3\right)$, representative of the cubic B20 family [an overview on the B20 series is given in Table I in the review by Nagaosa and Tokura (2013)], and tetragonal $\mathrm{Ba}_{2} \mathrm{CuGe}_{2} \mathrm{O}_{7}\left(P \overline{4} 2_{1} m\right)$ serve as two further examples where SANS was involved in key experiments. The weak itinerant FM MnSi exhibits a spin helix (Bak and Høgh Jensen, 1980). $\mathrm{Ba}_{2} \mathrm{CuGe}_{2} \mathrm{O}_{7}$ (Zheludev et al., 1996; Zheludev, Maslov et al., 1997) shows an AF spin cycloid.

A compact survey from the neutron scattering view on noncollinear spin structures is given by Chatterji (2006); see also Sandratskii and Kübler (1996), Sandratskii (1998), and Freeman and Nakamura (2004) for further reading. The recent impact of SANS on helical magnets and noncollinear spin structures is reviewed in Sec. VIII.A.5.

\section{Multiferroic properties of spiral magnets}

The multiferroic and magnetoelectric properties of noncollinear magnetic structures have lately gained remarkable interest, recently reviewed by Kimura $(2007,2012)$ and Tokura and Seki (2010). A large wealth of multiferroic materials that exhibit spiral magnetic structures exists. Using symmetry arguments, Mostovoy (2006) showed that crystal structures without inversion symmetry generally allow a coupling of a uniform polarization $\mathbf{P}$ to an inhomogeneous magnetization $\mathbf{M}$ that is linear in $\mathbf{P}$ and contains a gradient of $\mathbf{M}$ (Lifshitz invariant). It is exactly this Lifshitz invariant that also allows for the DMI, leading to a particularly strong coupling of ferroelectric and spiral magnetic properties (Katsura, Nagaosa, and Balatsky, 2005; Mostovoy, 2006; Sergienko and Dagotto, 2006). Within the DMI model, the antisymmetric interaction on the noncentrosymmetric bond causes a canting of spins which may generate uniform electric polarization. Within the inverse DMI scenario, a canted arrangement caused, e.g., by frustration, may displace the ion in between the spin sites and generate a new DMI vector or local polarization. Typical examples include hexagonal $\mathrm{Ba}_{0.5} \mathrm{Sr}_{1.5} \mathrm{Zn}_{2} \mathrm{Fe}_{12} \mathrm{O}_{22}$ (Kimura, Lawes, and Ramirez, 2005), perovskite $\mathrm{TbMnO}_{3}$ (Kenzelmann et al., 2005), and related $R \mathrm{MnO}_{3}(R=\mathrm{Y}, \mathrm{Ho}, \mathrm{Er}, \mathrm{Tm}, \mathrm{Yb}$, and Lu) (Fiebig et al., 2000), the kagome lattice $\mathrm{Ni}_{3} \mathrm{~V}_{2} \mathrm{O}_{8}$ (Lawes et al., 2005) and the rhombohedral $R 3 c$ perovskite $\mathrm{BiFeO}_{3}$ (Ramazanoglu et al., 2011).

\section{Noncollinear magnetism and diffraction in SANS geometry}

Magnetic SANS ideally suits the wavelengths of noncollinear spin structures of typically 30-1000 ̊. However, in contrast to diffuse SANS, based on the definition of a SLD which varies smoothly on an atomic scale (see Sec. II.B), an approach based on magnetic single-crystal diffraction is typically used for noncollinear spin structures [a detailed derivation is given by Squires (1978), Lovesey (1984), and Furrer, Mesot, and Strässle (2009)]. We start with the general cross section for elastic magnetic neutron diffraction of unpolarized neutrons on a single-crystalline sample with negligible mosaicity. Considering only magnetic scattering, the cross section reads 


$$
\begin{aligned}
\frac{d \Sigma}{d \Omega}= & \left(\gamma_{n} r_{0}\right)^{2} e^{-W(\mathbf{q})} f^{2}(\mathbf{q}) \sum_{\alpha, \beta}\left(\delta_{\alpha, \beta}-\frac{q_{\alpha} q_{\beta}}{q^{2}}\right) \\
& \times \sum_{l} e^{i \mathbf{q} \cdot \mathbf{l}}\left\langle\hat{S}_{0}^{\alpha}\right\rangle\left\langle\hat{S}_{l}^{\beta}\right\rangle
\end{aligned}
$$

with the spin operators $\left\langle\hat{S}_{0}^{\alpha}\right\rangle$, and $\alpha, \beta=x, y, z\left(\gamma_{n}=1.913\right.$ and $\left.r_{0}=2.818 \mathrm{fm}\right)$. The dimensionless magnetic form factor $f(\mathbf{q})$ is the Fourier transform of the normalized spin density associated with the magnetic ions (not to be confused with the form factor used in the classical, diffuse SANS theory), and $e^{-W(\mathbf{q})}$ denotes the temperature-dependent Debye-Waller factor. The sum over $l$ embodies the integration over all unit cells of a sample with $\mathbf{l}=\mathbf{R}_{j}-\mathbf{R}_{j^{\prime}}$ and atomic positions $\mathbf{R}_{j}$.

We assume the general form of a spin helix with propagation vector $\mathbf{k}$ (corresponding to a wavelength $k=2 \pi / \lambda_{\mathrm{h}}$ ) along the $z$ axis and spins lying in the $x-y$ plane. The expectation values of the spin operators are given by

$$
\begin{aligned}
\left\langle\hat{S}_{l}^{x}\right\rangle & =\langle\hat{S}\rangle \cos (\mathbf{k} \cdot \mathbf{l}), \\
\left\langle\hat{S}_{l}^{y}\right\rangle & =\langle\hat{S}\rangle \sin (\mathbf{k} \cdot \mathbf{l}), \quad\left\langle\hat{S}_{l}^{z}\right\rangle=0 .
\end{aligned}
$$

Different choices of $S$ and $\mathbf{k}$ may reproduce alternative magnetic structures with different periodicities, including multiple $\mathbf{k}$ and higher harmonics of $\mathbf{k}$. Inserting Eq. (62) into Eq. (61) finally leads to (Squires, 1978)

$$
\begin{aligned}
\frac{d \Sigma}{d \Omega}= & \frac{N}{4} \frac{(2 \pi)^{3}}{v_{0}}\left(\gamma_{n} r_{0}\right)^{2} e^{-W(\mathbf{q})} f^{2}(\mathbf{q})\langle\hat{S}\rangle^{2}\left(1+q_{z}^{2} / q^{2}\right) \\
& \times \sum_{\boldsymbol{\tau}}[\delta(\mathbf{q}+\mathbf{k}-\boldsymbol{\tau})+\delta(\mathbf{q}-\mathbf{k}-\boldsymbol{\tau})] .
\end{aligned}
$$

Bragg scattering occurs for $\mathbf{q}=\boldsymbol{\tau} \pm \mathbf{k}$, where $\boldsymbol{\tau}$ is a reciprocal lattice vector of the crystal. Each nuclear Bragg reflection is accompanied by a pair of two incommensurate satellite reflections at $k=2 \pi / \lambda_{\mathrm{h}}$. As SANS is capable of mapping reciprocal space in a limited region around the forward direction $\boldsymbol{\tau}=(000)$, incommensurate reflections are seen directly at $\pm \mathbf{k}$. The transformation from real to reciprocal space of a smooth modulation on top of a Bravais lattice is illustrated in Figs. 31(b1)-31(b3).

In contrast to diffuse SANS, incommensurate magnetic satellites represent Bragg reflections that are seen in the SANS geometry, leading to important consequences: Noncollinear magnetic structures may be generally seen as a modulation on top of a FM or AF, depending on the leading terms $J_{1}$ in their Hamiltonian [see Fig. 31(a)]. In the latter case, the rotation angle $\alpha_{r}$ can be rewritten as $\alpha_{r}+\pi / 2$; the staggered $\mathrm{AF}$ magnetization is used as an order parameter. For AF spin spirals, incommensurate magnetic satellites appear in pairs centered around the AF-Néel point [typically $(\pi, \pi)$ ]. As the direct beam $[\tau=(000)]$ corresponds to a FM zone center, only the FM components of any noncollinear magnetic structure are visible by SANS. A typical case is illustrated in Figs. 31(c) and 31(d): A small canting of an otherwise purely AF cycloidal spin structure of only $1^{\circ}$ leads to small FM correlations (corresponding to an amplitude of the FM SDW of only $\left.0.09 \mu_{B} / \mathrm{Fe}\right)$. This causes a SANS signal in ferroelectric $\mathrm{BiFeO}_{3}$ (Ramazanoglu et al., 2011). As the magnetic form factor of the corresponding ions $f(\mathbf{q}) \approx 1$ for small $\mathbf{q}$ around $(0,0,0)$, SANS is very sensitive to small FM modulations.

Typically dealing with single-crystal samples, the magnetic selection rules apply as for every other magnetic neutron diffraction experiment: Only components of $S$ perpendicular to $\mathbf{q}$ lead to a scattering signal [see Eq. (63)].

Further consequences of diffraction in the SANS geometry concern the coherence volume, which corresponds to a flat ellipsoid with the shortest half axis along the beam direction for diffuse SANS, caused by the coarse wavelength resolution $\Delta \lambda / \lambda$ (typically 10\%) (Felber et al., 1998). In perpendicular direction the coherence length is mainly governed by the divergence of the neutron beam. By contrast, Grigoriev et al. (2010) showed that the coherence volume of diffraction in the SANS geometry on objects with a two-dimensionally ordered nanostructure and a third nonperiodic dimension can go beyond the Born approximation: The Bragg reflection at the sample effectively acts as a neutron monochromator and leads to exceptionally elongated coherence lengths along the beam direction up to the $\mu \mathrm{m}$ regime. This is particularly relevant for well-ordered periodic structures, e.g., vortex lattices (Yaron et al., 1994) or SkLs (Adams et al., 2011). Vice versa, the coherence lengths of the magnetically ordered state can be deduced from the inverse peak widths of such diffraction peaks, $\xi=2 \pi / \Delta q$ corrected for instrumental resolution.

Similar to single-crystal diffraction, diffraction in the SANS geometry requires the use of goniometers or rotation stages to rotate the reciprocal lattice of single-crystal samples through the Ewald sphere. Despite the Bragg angles and associated rocking angles being small, helical magnets, skyrmion lattices, and also superconducting vortex lattices can yield exceptionally sharp rocking curves similar to the resolution limit of a SANS instrument at full collimation, typically $0.1^{\circ}$ (Adams et al., 2011). To capture the full intensity of a Bragg peak, it is always necessary to record the integrated intensity of a rocking curve. As a further complication, the existence of strong Bragg peaks may sometimes lead to a significant amount of double scattering, which can be easily confused with true higher harmonic terms at multiplies of $\mathbf{k}$ (Adams et al., 2011). By means of Renninger scans (the sample is rotated around the scattering vector $\mathbf{Q}$ ) (Shirane, Tranquada, and Shapiro, 2002) it is possible to discriminate true higherorder intensity from double scattering.

As spiral magnets often exhibit intrinsic magnetic chirality, the use of polarized neutrons, polarization analysis, and even 3D polarimetry can be beneficial for the unambiguous identification of magnetic structures and the separation of magnetic and nuclear scattering. The cross sections for polarized neutron scattering on chiral magnetic structures were derived by Maleev (1961), Izyumov and Maleev (1962), Blume (1963), and Maleev, Bar'yakhtar, and Suris (1963). An introduction to 3D polarimetry was given by Brown (2006). The papers by Kindervater et al. (2014) and Janoschek et al. (2007) provide typical examples.

\section{Properties of B20 spiral magnets as inferred from SANS}

A large body of SANS studies has been performed on the B20 family with $\mathrm{MnSi}\left(P 2{ }_{1} 3\right)$ as a drosophila in many regards 
(the skyrmonic spin textures found in these systems will be covered in Secs. VIII.B.1 and VIII.B.2). While all B20 compounds share a generic hierarchy of energy scales and the phase diagram as introduced in Sec. VIII.A.2, their electronic properties, pitch lengths, and transition temperatures vary considerably; a compact summary over the characteristic properties of the B20 series was given by Nagaosa and Tokura (2013) in Table I of their review article. It was pointed out by Manyala et al. (2004) that the concentration series $\mathrm{Fe}_{1-x, y} \mathrm{Mn}_{x} \mathrm{Co}_{y} \mathrm{Si}$ enables studying the continuous evolution from a classic weak itinerant FM, to a metallic paramagnet (PM), to a Kondo insulator and finally a polarized itinerant magnetic metal. The corresponding concentration-temperature phase diagram is given in Fig. 32(a). Finally, isostructural $\mathrm{Cu}_{2} \mathrm{OSeO}_{3}$ is an insulator with multiferroic properties (Seki, Yu et al., 2012), where a ferrimagnetic arrangement of $\mathrm{Cu}_{4}$ tetrahedra with a total spin moment of $S=1$ atoms takes over the role of the magnetic ion (Bos, Colin, and Palstra, 2008; Seki, Yu et al., 2012).

The generic phase diagram representative of the B20 series is shown in Fig. 32(b). Its archetypal example MnSi is a weak itinerant FM with a magnetic moment of $0.4 \mu_{B}$ per Mn atom and a fluctuating moment of $2.2 \mu_{B}$ (Lonzarich and Taillefer, 1985). In the helical phase below $T_{C}=29.5 \mathrm{~K}$ and below $H_{c 1} \approx 0.1 \mathrm{~T}$, a large body of SANS studies established a monochiral helical order, e.g., by Ishikawa et al. (1976), Hansen (1977), Ishikawa and Arai (1984), Lebech et al. (1995), and Grigoriev et al. (2005, 2006a, 2006b), 2007) with
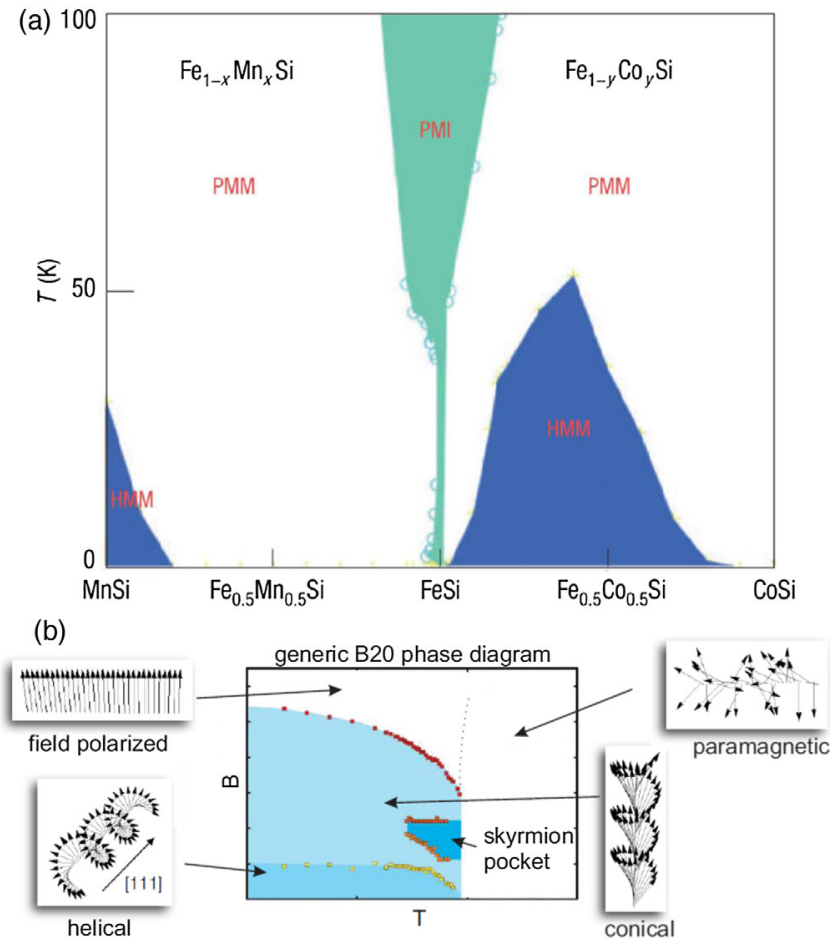

FIG. 32. (a) Concentration-temperature phase diagram of the isostructural series $\mathrm{Fe}_{1-x} \mathrm{Mn}_{x} \mathrm{Si}$ to $\mathrm{Fe}_{1-y} \mathrm{Co}_{x} \mathrm{Si}$. The abbreviations HMM, PMM, and PMI denote helimagnetic metal, PM metal, and PM insulator, respectively (Manyala et al., 2004) (b) Generic B20 phase diagram with an illustration of the characteristic main phases. From Markus Garst. a wavelength of $180 \AA$ caused by DMI. The propagation vector $\mathbf{k}$ aligns along the (111) directions due to second-order anisotropy terms (Bak and Høgh Jensen, 1980). For increasing magnetic fields $\mathbf{k}$ realigns in the direction of $\mathbf{H}$, characteristic of a spin flop transition and the helix deforms to a cone phase (Ishikawa et al., 1976), until a fully field-polarized state is reached at the upper critical field $H_{c 2}$. The length of the pitch only weakly depends on temperature or magnetic field. Bulk measurements (Bauer and Pfleiderer, 2012) showed that, due to demagnetizing effects, phase coexistence and extended crossover regions can be observed.

An extended region dominated by fluctuations is observed around the transition to the PM phase below a tricritical point (Bauer, Garst, and Pfleiderer, 2013), which was studied by means of SANS (Janoschek et al., 2013): As a consequence of the small propagation vector $\mathbf{k}$, magnetocrystalline anisotropies are less effective in fixing the orientation of the magnetic order. Depending on the crystalline symmetry of the material, the energies of spiral magnetic textures might then be almost degenerate for orientations of $\mathbf{k}$ that belong to manifolds in momentum space, e.g., a sphere or a ring. It was proposed by Brazovskii (1975) that such a manifold may qualitatively alter the nature of a phase transition: Upon approaching the phase transition from high $T$, magnons soften on this manifold at finite $\mathbf{k}$, giving rise to a large phase space. The abundance of fluctuations results a substantial magnetic entropy. To avoid this entropy the phase transition is driven to first order. Based entirely on symmetry arguments, this scenario was discussed in various contexts, e.g., for weak crystallization (Brazovskii, Dzyaloshinskii, and Muratov, 1987), liquid crystals (Brazovskii and Dmitriev, 1975; Swift, 1976), diblock copolymers (Leibler, 1980; Bates, Rosedale, and Fredrickson, 1990), and Bose-Einstein condensates (Gopalakrishnan, Lev, and Goldbart, 2009).

Recent SANS experiments by Janoschek et al. (2013) showed that such a scenario is realized in MnSi with magnons softening on a sphere in $\mathbf{k}$ space. Typical data are shown in Fig. 33. Figure 33(a) shows the evolution from sharp diffraction peaks associated to the helical domains $\left(T<T_{C}\right)$, to a diffuse ring, representating a cut through a sphere $\left(T>T_{C}\right)$. Figure 33(b) shows the temperature dependence of the inverse correlation length $\kappa$ as inferred from SANS and measurements of the susceptibility. Subsequently, it was demonstrated by means of susceptibility and SANS that other members of the $\mathrm{B} 20$ series such as $\mathrm{Cu}_{2} \mathrm{OSeO}_{3}$ (Živković et al., 2014) show similar behavior. However, SANS and spin-echo studies by Bannenberg et al. (2017) on $\mathrm{FeCo}_{1-x} \mathrm{Si}_{x}$ revealed a more complex behavior with a coexistence of fluctuations and helimagnetic order over a broad temperature interval and long relaxation times with a stretched exponential that persists even under magnetic field.

In summary, these studies and related spin-echo measurements on MnSi (Pappas et al., 2009) essentially demonstrate an important feature of noncollinear magnets: The length scale of $\mathbf{k}$ is still present in the characteristic fluctuations of incommensurate spiral magnets well above $T_{C}$ in the PM regime.

Similarly in the field-polarized FM state of a chiral spin structure such as $\mathrm{MnSi}$, the spin-wave dispersion is distinct from a conventional FM. Because of the DMI, the spin-wave 

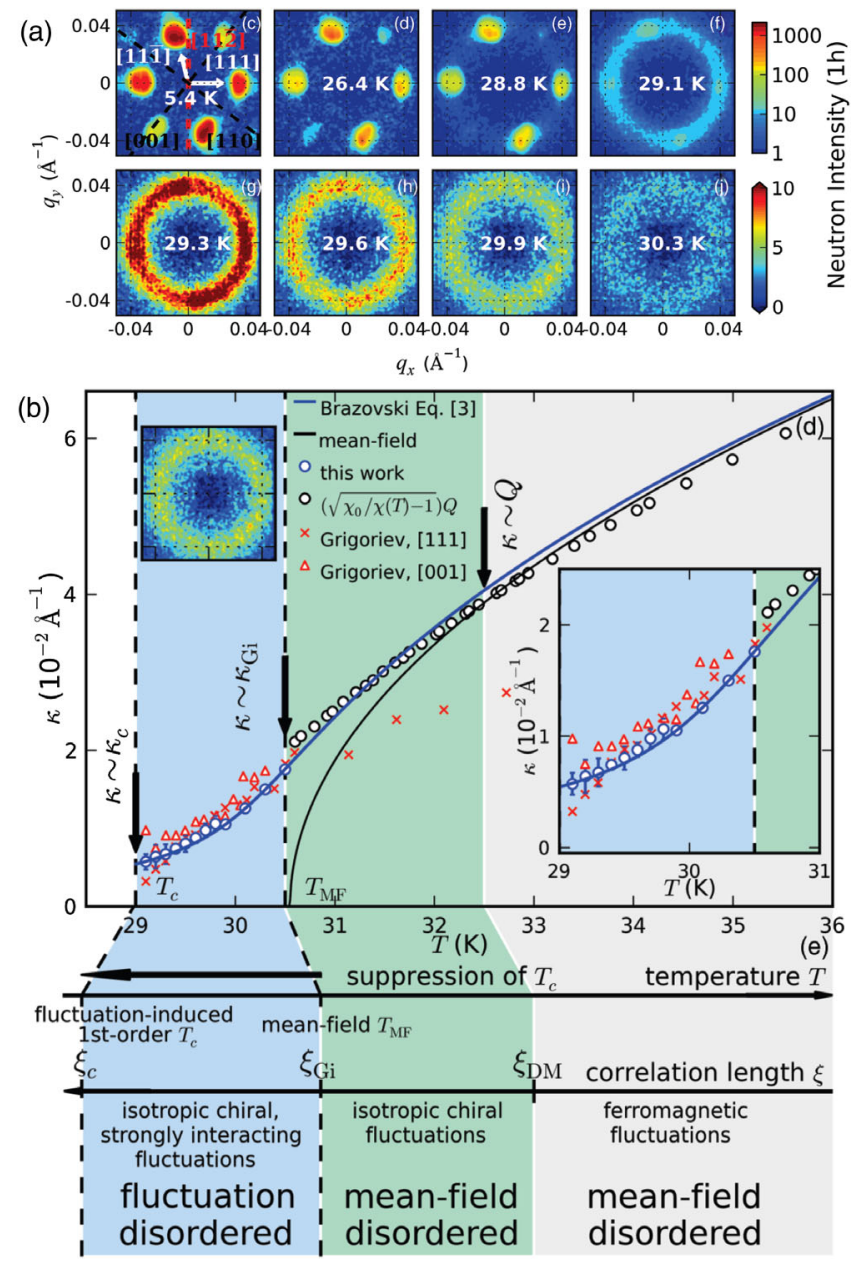

FIG. 33. (a) Evolution of sharp diffraction peaks associated with the helical domains, observed below $T_{C}$, to a diffuse ring, representative for a cut through a sphere for temperatures above $T_{C}$. (b) Characteristic temperature of the inverse correlation length $\kappa$ as inferred from SANS and measurements of the susceptibility. From Janoschek et al., 2013.

dispersion is centered at $\pm \mathbf{k}$ instead of $q=0$ (Kataoka, 1987). The antisymmetric interaction of polarized neutrons with the chiral spin structure of MnSi and related materials has been used in a series of SANS experiments by Grigoriev et al. (2015) and Siegfried et al. (2017) to measure the spin-wave stiffness in the spirit of a similar approach made earlier for FM spin waves (Toperverg, Deriglazov, and Mikhailova, 1993). This method is particularly helpful for materials such as FeGe, where high-pressure synthesis allows only the growth of tiny crystallites or powders which do not allow the use of typical inelastic neutron instruments such as triple-axis spectrometers (Kugler et al., 2015). Moreover, it benefits from the superior resolution of SANS instruments at small k, which are otherwise challenging to resolve. In the field-polarized states of MnSi (Grigoriev et al., 2015) and FeGe (Siegfried et al., 2017), SANS shows intensity in a circle, centered at $\pm \mathbf{k}$. The radius or critical angle $\theta_{c}$ of the circle allows deducing the spin-wave stiffness. For this method, the magnetic field is aligned perpendicular to $\mathbf{k}_{0}$. Note that for FM spin waves the magnetic field needs to be tilted with respect to $\mathbf{k}_{0}$ as these do not show the inherent chirality of helical magnets.
A further point addressed in the B20 series concerns the coupling of crystalline and magnetic chirality. While the absolute chirality of the crystalline structure of the B20 monosilicides of $\mathrm{Fe}, \mathrm{Co}$ and $\mathrm{Mn}$ is randomly governed by crystal growth, the relative chirality of the helices with respect to the crystal is given by the orientation and the sign of $\mathbf{D}$. The handedness of both the magnetic helix and crystal can be addressed by means of polarized SANS and single-crystal $\mathrm{X}$-ray diffraction, respectively. Measurements on the concentration series of $\mathrm{Mn}_{1-x} \mathrm{Fe}_{x} \mathrm{Ge}$ (Grigoriev et al., 2013), $\mathrm{Fe}_{1-x} \mathrm{Co}_{x} \mathrm{Ge}$ (Grigoriev et al., 2014), and $\mathrm{Fe}_{1-x} \mathrm{Co}_{x} \mathrm{Si}$ (Siegfried et al., 2015) reveal a breakdown of the helical order with vanishing $\mathbf{k}$ associated with a flip of relative chirality and reentrant FM behavior at the critical concentration $x_{c}$. Consequently, opposite relative chirality is observed for opposite ends of each concentration row. The microscopic reason for the associated change of sign of the DMI is not resolved unambiguously, although it has been discussed that a competition of DMI and cubic anisotropy might play a role. Recent ab initio theoretical calculations by Koretsune, Nagaosa, and Arita (2015) showed that the behavior of DMI can be systematically understood in terms of the details of the electronic band structure.

Besides a systematic tuning of pitch length, relative chirality, $T_{C}$, and electronic properties, the concentration series of the B20 members allows for systematic studies of quantum criticality and the effects of disorder. Bulk and SANS measurements by Bauer et al. (2010) and Kindervater et al. (2018) revealed a critical concentration of $x_{c}^{\mathrm{Fe}}=0.192$ and $x_{c}^{\mathrm{Co}}=0.084$ where the transition temperature vanishes for single crystals of $\mathrm{Mn}_{1-x} \mathrm{Fe}_{x} \mathrm{Si}$ and $\mathrm{Mn}_{1-x} \mathrm{Co}_{x} \mathrm{Si}$, respectively. The behavior of both concentration series agrees on a normalized doping scale $x / x_{c}$, suggesting the existence of a putative quantum-phase transition, dominated by FM quantum fluctuations. Recent SANS measurements on polycrystalline $\mathrm{Mn}_{1-x} \mathrm{Fe}_{x}$ Ge by Altynbaev et al. (2016), and on powders of $\mathrm{Mn}_{1-x}(\mathrm{Co}, \mathrm{Rh})_{x}$ Ge by Martin et al. (2017), show a far more complex behavior, although the influence of crystalline quality remains unclear.

Alternative to doping, pressure serves as another tuning parameter. The transition temperature of MnSi is suppressed until it reaches $T_{C}=0$ at $p_{c}=15 \mathrm{kbar}$ and the phase transition thereby changes from second order to weak first order at $p^{\star}=$ 12 kbar (Pfleiderer, McMullan, and Lonzarich, 1995; Pfleiderer et al., 2007). An extended region of non-Fermiliquid behavior emerges above $p_{c}$ (Schofield, 1999; Pfleiderer, Julian, and Lonzarich, 2001; Doiron-Leyraud et al., 2003), characterized by an unusual exponent of the electrical resistivity $\rho(T) \propto T^{\alpha}$ with $\alpha=3 / 2$. SANS and neutron diffraction observed a weak partial magnetic order above $p^{\star}$ (Pfleiderer et al., 2004, 2007), where the spontaneous formation of a skyrmionic ground state at zero field was discussed by Rößler, Bogdanov, and Pfleiderer (2006). Recent measurements by Ritz et al. (2013) connected the topological Hall signal and the non-Fermi-liquid regime, suggesting the presence of disordered structures with nontrivial topology.

\section{Properties of non-B20 spiral magnets as inferred from SANS}

The noncentrosymmetric tetragonal AF $\mathrm{Ba}_{2} \mathrm{CuGe}_{2} \mathrm{O}_{7}$ $\left(P \overline{4} 2{ }_{1} m\right)$ shows a long-range incommensurate, AF cycloidal 
spin spiral due to DMI below $T_{N} \approx 3.05 \mathrm{~K}$ of which a number of neutron scattering studies [see, e.g., Zheludev, Shirane et al. (1997) and Mühlbauer et al. (2012) and references therein] and theoretical work (Bogdanov et al., 2002; Chovan, Papanicolaou, and Komineas, 2002; Chovan, Marder, and Papanicolaou, 2013) have established a quite complete understanding. However, it was predicted that a second sign alternating component of the DMI vector pointing along the $c$ axis should give rise to incommensurate weak FM behavior (Chovan, Papanicolaou, and Komineas, 2002; Chovan, Marder, and Papanicolaou, 2013) with the same k. This prediction was confirmed by SANS (Mühlbauer et al., 2017). A similar situation observed in the ferroelectric AF $\mathrm{BiFeO}_{3}$ has already been illustrated in Sec. VIII.A.4. Here polarization analysis was used in addition to separate nuclear from magnetic scattering.

$\mathrm{Ba}_{2} \mathrm{CuGe}_{2} \mathrm{O}_{7}$ is also relevant in terms of a Brazovskii scenario (Brazovskii, 1975), however, with reduced 2D symmetry caused by the weak interplane coupling of $\mathrm{Ba}_{2} \mathrm{CuGe}_{2} \mathrm{O}_{7}$ : Because of crystallographic anisotropy, the manifold of fluctuations is reduced to a ring in reciprocal space. SANS and neutron diffraction have been used to shed light on the interplay of Brazovskii and 2D physics (Mühlbauer et al., 2017).

The ternary compound $\operatorname{Pr}_{5} \mathrm{Ru}_{3} \mathrm{Al}_{2}$ crystallizes in the cubic noncentrosymmetric and nonmirror-symmetric space group $I 2_{1} 3$, hence allowing for DMI. Indeed, a study by Makino et al. (2016) on high quality powder samples indicated the existence of incommensurate peaks at $\mathbf{k} \approx(0.066,0.066$, 0.066) (r.l.u.) below $T_{N}=3.8 \mathrm{~K}$, while the FM transition found earlier was attributed to impurity phases. These incommensurate peaks have been confirmed by means of SANS measurements (Okuyama et al., 2017). Representation analysis was used to propose a helical spin structure of a composed moment of the different Pr layers.

The rare-earth metal Ho shows various spiral magnetic phases, generated by an oscillatory RKKY interaction of the localized $4 f$ spins through the conduction electrons. Between the Néel temperature $T_{N}=132 \mathrm{~K}$ and the Curie point $T_{C}=20 \mathrm{~K}$, an AF helical phase is found by neutron diffraction for single-crystal samples. Below $T_{C}$, an additional FM component gives rise to a conical phase. Upon increasing magnetic field, the spin structure shows a series of transitions to a helifan(3/2), a helifan(2), and finally a fan structure for field along the $b$ axis (Jensen and Mackintosh, 1990; Kosugi et al., 2003). Helifan denotes a mixture of helix and fan each with different periodicities. SANS experiments have been used to study the influence of grain size on the spin structures of Ho and related Tb (Michels et al., 2011; Szary et al., 2016). In contrast to single-crystalline samples, Szary et al. (2016) found no evidence of the helifan structures in nanocrystalline Ho by means of field-dependent SANS. For coarse grained samples, a streak pattern indicative of a long-period magnetic structure is observed, correlated with the vanishing of the helix for increasing field.

The members of the series $R \mathrm{Mn}_{6} X_{6}$, where $R$ is a rare-earth element and $X$ is a metal with an unfilled $p$ shell exhibit a hexagonal, layered structure and show a large diversity of magnetic phases, generated by competition of FM exchange between the $X-X-X$ planes, AF coupling via the $R-X$ planes, and finally RKKY exchange between the next-nearestneighbor Mn planes. For $\mathrm{YMn}_{6} \mathrm{Sn}_{6}$, SANS studies by Bykov et al. (2015) found a helical ordering with $\mathbf{k}=1.7 \mathrm{~nm}^{-1}$ that gives way to a state with spatial FM fluctuations at zero wave vector in the interval of $250-360 \mathrm{~K}$ and finally leads to a PM regime. Since the system is strongly anisotropic with the moments confined in the (001) plane, the phase transition is considered to be quasi two dimensional. A qualitatively similar behavior was observed by Altynbaev et al. (2016) in powder samples of B20 MnGe (crystallite size $1 \mu \mathrm{m}$ ). Here a Gaussian peak at incommensurate $2 \mathrm{~nm}^{-1}$, associated with static helical order, is continuously replaced by a Lorentzian peak at the same $\mathbf{k}$ indicative of fluctuations for increasing temperature. At $T_{N}=130 \mathrm{~K}$, the Lorentzian contribution dominates. Both contributions exist above $T_{N}$ and show exponentially activated behavior. Well above $T_{N}$, additional Gaussian correlations are observed at lower q, attributed to static short-range FM inhomogeneities. The phase transition observed for $\mathrm{MnGe}$ is interpreted as a complex order-to-disorder transition, although the influence of the crystallite size remains unclear.

The bond frustrated compound $\mathrm{ZnCr}_{2} \mathrm{Se}_{4}$ [cubic spinel $(F d \overline{3} m)$ structure] is magnetoelectric and shows a strong spin-lattice coupling. An AF spiral with a screw angle of $42^{\circ}$ is observed below $T_{N}=20 \mathrm{~K}$ that goes along with a structural transition to tetragonal symmetry (Felea et al., 2012). SANS (Cameron et al., 2016) has been used to study the field dependence of the multidomain spin spiral phase with particular focus on a spin-nematic phase, proposed at higher field (Felea et al., 2012). A continuous change of magnetic structure is observed as a function of field and temperature while a discontinuous jump of the spiral pitch indicates the domain selection field. Most importantly, no signs of long-range order have been found in the high-field spin-nematic phase.

\section{B. Magnetic skyrmions}

\section{The concept of skyrmions}

The concept of skyrmions goes back to British physicist Tony Skyrme (Skyrme, 1961a, 1961b, 1962) and later contributions by Adkins, Nappi, and Witten (1983), who managed to show that in the presence of nonlinear coupling, excitations of continuous fields can assume particlelike character. Skyrmionic states are characterized by a nontrivial topology, which can be conveniently classified by means of winding numbers (Manton and Sutcliffe, 2004). A nonzero, integer winding number describes a topology that cannot be smoothly distorted to a trivial state and, as a consequence, leads to countable entities (e.g., a vortex state in 2D). In turn, the topological properties are intimately connected to particle stability. Originally developed to explain the constituents of the nucleus, protons, and neutrons, as topological excitations of a spinless pion field, the concept of skyrmions has been used in different physical contexts. In condensed matter physics, skyrmionic states have been described in quantum Hall magnets (Sondhi et al., 1993) at finite magnetic field and in topological insulators at zero field (Konig et al., 2007; Hsieh et al., 2008). Recent reviews of the general concept of skyrmions have been given by Manton and Sutcliffe (2004) and Brown and Rho (2010). 
In their seminal studies Bogdanov and Yablonskii (1989), Bogdanov and Hubert (1994), Bogdanov (1995), and Bogdanov and Rößler (2001) predicted the stabilization of magnetic vortices by Bloch domain walls in anisotropic noncentrosymmetric FMs, similar to superconducting vortices which are stabilized by the negative energy of a normal to superconducting interface. If these vortex states in bulk magnets have a nontrivial winding density, they can be regarded as skyrmions. Smectic, nematic, and hexatic forms of order, akin to liquid crystals and multi-k structures have been discussed for spin liquids or spin glasses found in frustrated systems such as, e.g., kagome lattices or 3D pyrochlore AFs (Forgan et al., 1989; Lawler et al., 2008); however, their topological properties have not been discussed in detail.

Schematic depictions of the spin structures of a Néel-type and a Bloch-type single skyrmion are shown in Figs. 34(a) and 34(b). Note that both exhibit similar topological properties or winding numbers. Figure 34(c) shows the arrangement of skyrmions to a SkL with hexagonal symmetry.

The first experimental indication of magnetic skyrmions in helimagnetic MnSi revealed magnetic whirls that arrange in a SkL (Mühlbauer, Binz et al., 2009). SANS established a wellordered Bloch-type SkL phase pocket (formerly denoted $A$ phase) at $\approx 0.2 \mathrm{~T}$, close to $T_{C}$ in $\mathrm{MnSi}$. A resolution limited hexagonal scattering pattern akin to a superconducting vortex lattice, aligned perpendicular to the magnetic field irrespective of the crystal orientation (Mühlbauer, Binz et al., 2009) is
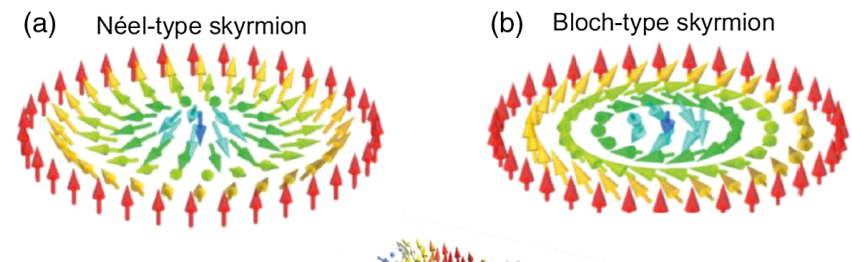

(c) skyrmion lattice

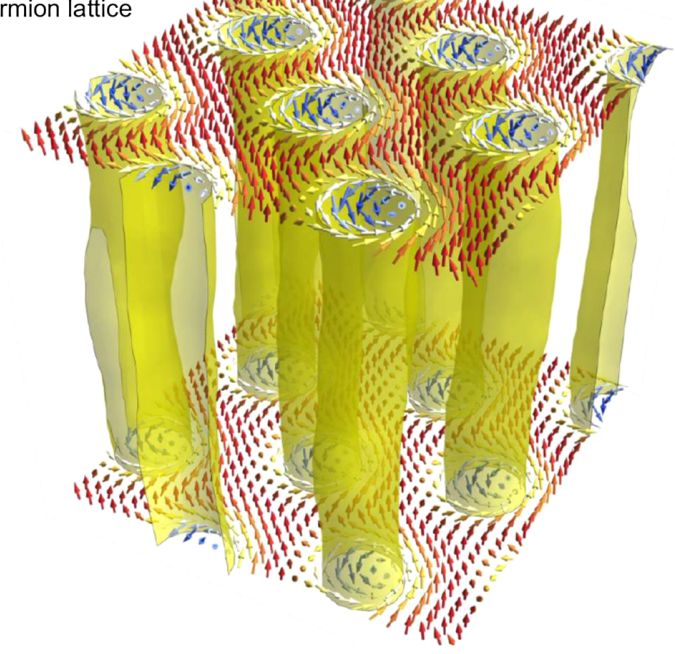

FIG. 34. (a), (b) Schematic depictions of a Néel-type and a Bloch-type single skyrmion. A cut through a single skyrmion yields the spin structure of a Néel and a Bloch domain wall, respectively. From Markus Garst. (c) Schematic depiction of a skyrmion lattice with the hexagonal arrangement of skyrmion lines through the sample. From Milde et al., 2013. observed. The spin structure is translationally invariant in the direction of the magnetic field, leading to skyrmion lines. A Ginzburg-Landau ansatz based on a triple-k state including Gaussian fluctuations in the spirit of an order-by-disorder mechanism qualitatively reproduced the stable region of the SkL phase in the $B, T$-phase diagram [Fig. 35(a)] and showed that the SkL state corresponds to the thermodynamic minimum. Typical SANS data from MnSi are shown in Figs. 35(b) and 35(c).

Characterized by a nonzero winding number of -1 , these whirls have skyrmionic character. Their topology is distinct from the surrounding conical phase, leading to particlelike characteristics of single skyrmions. Since SANS does not couple directly to the topology of the SkL state (single-k multidomain versus multi-k single domain), its topological properties have been probed by Hall measurements by Neubauer et al. (2009). Besides the normal and anomalous Hall effect, an additional signal appears, caused by the Berry phase which the moving conduction electrons acquire as their spin follows the nontrivial topology of the SkL. Since then the

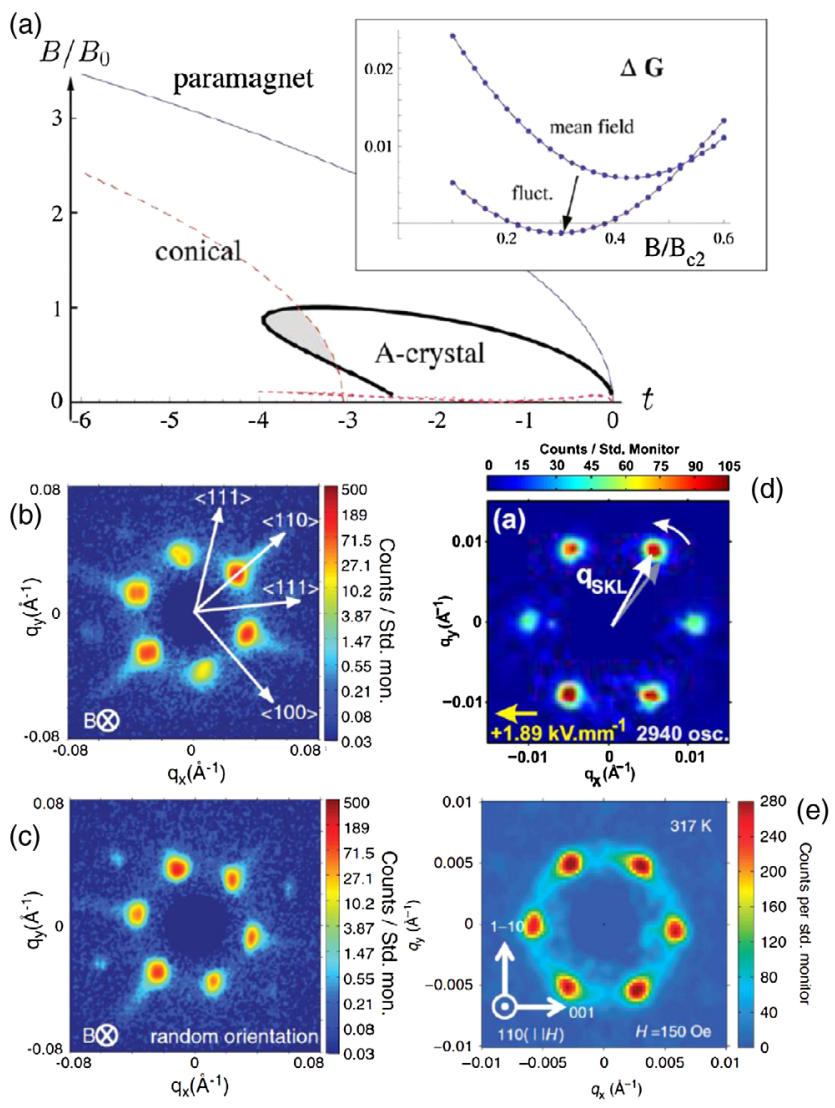

FIG. 35. (a) The phase diagram of MnSi calculated by means of a Ginzburg-Landau ansatz based on a triple- $k$ state. The inset shows the effect of Gaussian fluctuations on lowering the free energy of the SkL phase. (b), (c) SANS data of the SkL in MnSi for a magnetic field parallel to (b) (110) and (c) a random direction of the magnetic field (Mühlbauer, Binz et al., 2009). (d) A SANS pattern of the $\mathrm{SkL}$ in ferroelectric $\mathrm{Cu}_{2} \mathrm{OSeO}_{3}$ with magnetic field along the (100) axis (White et al., 2014). (e) SANS data of the SkL in a single-crystalline sample of $\mathrm{Co}_{8} \mathrm{Zn}_{8} \mathrm{Mn}_{4}$ with magnetic field along (110) (Tokunaga et al., 2015). 
topological Hall effect has been established as a hallmark of nontrivial skyrmionic structures (Ritz et al., 2013).

Further high-resolution SANS experiments showed an exceptionally long-range ordered SkL and were able to prove the existence of weak, higher-order reflections (i) indicative of the particlelike character of the SkL (Adams et al., 2011) and (ii) the fixed phase relation of the triple-k state. The pinning of the SkL to the crystal lattice in the plane perpendicular to the field is given by weak higher-order anisotropy terms, which have been examined in a series of SANS experiments (Adams, 2015; Adams et al., 2018) including the use of uniaxial pressure (Chacon et al., 2015). As for the helix state, the SkL state in MnSi is only weakly affected by crystal anisotropies, which reflects in an essentially isotropic temperature-field phase diagram and the particular coupling of the SkL spin structure to the magnetic field.

Inspired by the observation of magnetic skyrmions in $\mathrm{MnSi}$ and later in $\mathrm{FeCo}_{1-x} \mathrm{Si}_{x}$ (Mühlbauer, Binz et al., 2009; Münzer et al., 2010) by means of SANS, similar textures with topological properties have been observed in increasing number in various materials, including metals (Nagaosa and Tokura, 2013), semiconductors (Münzer et al., 2010), and insulators (Adams et al., 2012), again with SANS playing a pivotal role. The finding of skyrmions beyond the B20 family (Kézsmárki et al., 2015; Tokunaga et al., 2015; Bordacs et al., 2017; Kurumaji et al., 2017; Nayak et al., 2017) and, in particular, their observation in thinned bulk samples (Yu et al., 2011), thin magnetic films (Heinze et al., 2011), and nanostructured patterns (Boulle et al., 2016) using real-space methods such as magnetic force microscopy (MFM), spin-polarized STM, or Lorentz transmission electron microscopy (LTEM) have established magnetic skyrmions as a generic phenomenon of materials that support chiral interactions due to broken inversion symmetry. A general review of the properties of magnetic skyrmions was given by Bauer and Pfleiderer (2010) and Nagaosa and Tokura (2013). In analogy to superconducting vortex matter (Sec. IX), a similarly large variety of skyrmion matter is expected.

Note that SANS is only able to access skyrmionic matter of bulk samples that shows at least short-range order, e.g., SkLs, glasses, or liquids. The observation of individual skyrmions and their particlelike character (in particular for surfaces and thin film samples) is possible by the real-space methods mentioned in the previous paragraph.

\section{Skyrmions in spiral magnets seen by SANS}

SANS studies by Jonietz et al. (2010) showed that spin transfer torque effects allow for manipulation of the SkL at ultralow current densities of $\approx 10^{6} \mathrm{~A} \mathrm{~m}^{-2}$ facilitated by (i) the efficient decoupling of $\mathrm{SkL}$ and crystal lattice due to the smooth texture and (ii) the efficient coupling of the transport currents due to the topological properties of the SkL (Everschor et al., 2012). Complementary to measurements of spin transfer torque, recent time-resolved SANS measurements using the TISANE technique with periodically oscillating magnetic fields on MnSi (Mühlbauer, Kindervater et al., 2016) consistently show a depinning transition of the SkL at a critical oscillation amplitude. These measurements allow one to directly track the rigidity and pinning of the SkL also for nonconducting samples.
The stabilization of SkL phases is a central point of an increasing effort in experiment and theory: (i) Besides stabilization by fluctuations as found for MnSi (Mühlbauer, Binz et al., 2009), $\mathrm{Fe}_{1-x} \mathrm{Co}_{x} \mathrm{Si}$ (Münzer et al., 2010), FeGe (Yu et al., 2011), $\mathrm{Cu}_{2} \mathrm{OSeO}_{3}$ (Seki, Kim et al., 2012), and the $\beta$ Mn series (Tokunaga et al., 2015), several studies using realspace techniques revealed (ii) an increasing skyrmion phase with decreasing sample thickness (Yu et al., 2010, 2011; Seki, Yu et al., 2012). (iii) The influence of crystalline anisotropy has been investigated in several studies (Adams, 2015; Adams et al., 2018; Chacon et al., 2018); $\beta$-Mn type $\mathrm{Co}_{x} \mathrm{Zn}_{y} \mathrm{Mn}_{z}$ (Karube et al., 2016) exhibits a transition from a conventional triangular lattice to a metastable square SkL for low temperatures due to magnetocrystalline anisotropy. (iv) The destabilization of competing phases (Yu et al., 2010, 2011; Seki, Yu et al., 2012), (v) strain (Nii et al., 2015; Fobes et al., 2017), and (vi) terms induced by free surfaces (Rybakov et al., 2015) and interface spin-orbit effects (Heinze et al., 2011; Romming et al., 2013) play an important role, in particular, with decreasing sample thickness. Here SANS, GISANS, and polarized neutron reflectometry (PNR) have been used to study the possible formation of skyrmionic structures in B20 thin films: While such textures have been claimed to exist in MnSi thin films (Karhu et al., 2012; Wilson et al., 2013; Meynell et al., 2017), based on magnetization and PNR and SANS measurements, recent GISANS studies (Wiedenmann et al., 2017) did not reveal any hints for SkL spin textures in thin epitaxial films of MnSi.

As the SkL is topologically distinct from the surrounding conical phase, a continuous transformation of a conical state to a SkL is impossible or is at least associated with a large energy barrier. In combination with pinning induced by defects, this offers the study of considerable metastable, out-ofequilibrium, and hysteretic effects, which have been demonstrated by quenching the SkL into long-lived metastable phases in various compounds (Münzer et al., 2010; Karube et al., 2016; Oike et al., 2016; Nakajima et al., 2017). The topological difference raises the generic question of the process of skyrmion nucleation or decay at the borders of the SkL phase pocket, where a first-order phase transition is observed by bulk measurements (Bauer and Pfleiderer, 2012). SANS combined with real-space MFM measurements on $\mathrm{FeCo}_{1-x} \mathrm{Si}_{x}$ (Milde et al., 2013) showed that skyrmions decay by means of a topological defect that zips two neighboring skyrmions and propagates along the skyrmion line. Interestingly, the topological defect has the mathematical form of a monopole of emergent flux. In line with bulk and neutron imaging measurements (Bauer and Pfleiderer, 2012), geometry, demagnetization, and edge effects play an important role (Müller, Rosch, and Garst, 2016), suggesting that macroscopic phase coexistence is present and might have been underestimated (Reimann et al., 2018).

SANS experiments by Seki, Kim et al. (2012), Adams et al. (2012), and White et al. $(2012,2014)$ and LTEM studies by Seki, Yu et al. (2012) established the existence of a SkL in the multiferroic B20 insulator $\mathrm{Cu}_{2} \mathrm{OSeO}_{3}$. The phase diagram shares some qualitative features of all B20 compounds, although a (100) pinning of the helical order at zero field indicates that (i) the magnetocrystalline anisotropy terms are 
different and (ii) the difference of free energy for pinning along (100) and (110) is very small. The different anisotropy terms reflect in the orientation of the SkL, multidomain states are typically seen for $\mathrm{Cu}_{2} \mathrm{OSeO}_{3}$ (Adams et al., 2012; White et al., 2012) [see Fig. 35(d) for typical SANS data]. Moreover, a second independent skyrmion phase for $\mathbf{H} \|(100)$ at low temperature observed by means of SANS in $\mathrm{Cu}_{2} \mathrm{OSeO}_{3}$ is attributed to a stabilization mechanism invoking cubic anisotropy terms (Chacon et al., 2018).

The influence of the SkL to an electric field was investigated by White et al. (2012) in $\mathrm{Cu}_{2} \mathrm{OSeO}_{3}$ : For magnetic fields along a $(1, \overline{1}, 0)$ axis and electric field along (111) it is possible to induce small rotations of the SkL around the magnetic field axis. Consistent with symmetry arguments (Seki, Ishiwata, and Tokura, 2012) the SkL state permits a polar state for field along $(1,1,0)$, namely, $\boldsymbol{P} \|(001)$. Each electric dipole associated with a single skyrmion hence is inclined with respect to the field along $\boldsymbol{E} \|(111)$. The coupling of $P$ and $E$ is made responsible for the rotation of the SkL. It was found in a follow-up study (White et al., 2014) that electrical fields in combination with slight oscillations of the magnetic field amplitude help overcoming SkL pinning and lead to significantly larger rotation angles.

Recently, SANS studies have revealed the existence of SkL phases in an increasing number of non-B20 compounds. $\beta$-Mn type $\mathrm{Co}_{x} \mathrm{Zn}_{y} \mathrm{Mn}_{z},(x+y+z=20)$, crystallizes in the cubic, chiral $P 4_{1} 32$ space group. Accordingly, the ground state is a helical structure provided by DMI. SANS, magnetometry, and LTEM measurements by Tokunaga et al. (2015) in thin platelets and small bulk samples have identified a Bloch-type SkL with a phase diagram that shares similarities to cubic $\mathrm{MnSi}$, although the transition temperature is much higher $\approx 320 \mathrm{~K}$. Similar to $\mathrm{MnSi}$, the SkL is stabilized by an orderby-disorder mechanism.

A qualitatively different situation is found in polar $\mathrm{VOSe}_{2} \mathrm{O}_{5}$ (space group $C_{4 v}$ ), $\mathrm{GaV}_{4} \mathrm{~S}_{8}$, and $\mathrm{GaV}_{4} \mathrm{Se}_{8}$ (space group $C_{3 v}$ ), where the ground state is a cycloidal spin arrangement. Correspondingly, SANS, atomic force microscopy (AFM), electron spin resonance, and magnetization measurements have identified a Néel-type SkL in these materials (Kézsmárki et al., 2015; Bordacs et al., 2017; Kurumaji et al., 2017). In stark contrast to the cubic compounds, where weak anisotropies govern the orientation of the helical order and the SkL with respect to the crystal lattice and the SkL is essentially tied to the magnetic field as leading contribution, the SkL state is confined to certain crystallographic planes by crystal anisotropy for polar $\mathrm{GaV}_{4} \mathrm{~S}_{8}$ (Kézsmárki et al., 2015) and $\mathrm{GaV}_{4} \mathrm{Se}_{8}$ (Bordacs et al., 2017). Moreover, the competing conical state is forbidden by the DMI pattern in the polar $C_{v n}$ class. Accordingly, the phase diagram is qualitatively different, showing an extended region of stability of the SkL phase, although it is strongly dependent on the crystallographic direction (Kézsmárki et al., 2015; Bordacs et al., 2017). Antiskyrmion structures, characterized by alternating Bloch and Néel spin rotation following the boundary of the single skyrmion, have been identified in the acentric tetragonal Heusler compound $\mathrm{Mn}_{1.4} \mathrm{Pt}_{0.9} \mathrm{Pd}_{0.1} \mathrm{Sn}$ with $D_{2 d}$ symmetry by means of LTEM (Nayak et al., 2017).

The emergence of skyrmionic textures in magnetically frustrated centrosymmetric materials with high lattice symmetry has recently been the focus of intense theoretical effort (Okubo, Chung, and Kawamura, 2012; Leonov and Motovoy, 2015; Wang et al., 2015; Hayami, Ozawa, and Motome, 2017). Without the restrictions on the symmetry imposed by the DMI interaction, a large body of different helimagnetic structures and multiple-k modulations, based on frustration effects, remain to be investigated for their topological properties. Because of lesser restrictions on symmetry, skyrmions with topological charges 1 and 2 (Leonov and Motovoy, 2015), skyrmion-antiskyrmion lattices (Okubo, Chung, and Kawamura, 2012), and 3D modulated structures (Wang et al., 2015) are proposed. It is expected that neutron diffraction and SANS will play a key role in their identification.

\section{VORTEX LATTICES IN SUPERCONDUCTORS}

Superconductors are classified as type I or type II depending on their response to an applied magnetic field (Tinkham, 1996). Most superconducting materials, and almost all that are technologically relevant, belong to the second category. Subjecting a type-II superconductor to a magnetic field will introduce vortices, each carrying one quantum of magnetic flux $\Phi_{0}=h / 2 e=2068 \mathrm{~T} \mathrm{~nm}^{2}$ (Abrikosov, 1957; Huebener, 2001). The vortices introduce singularities in the order parameter and may be used as probes of the superconducting state in the host material. Moreover, moving vortices give rise to dissipation within the superconducting state, and the dynamical properties of vortex matter constitutes its own important area of research (Blatter et al., 1994; Brandt, 1995).

\section{A. Imaging the vortex lattice by neutron diffraction}

Because of their mutual repulsion, vortices arrange themselves in an ordered VL, as long as the vortex-vortex interactions dominate external influences such as pinning to impurities or thermal disordering. The VL gives rise to a periodic magnetic field modulation and may therefore be imaged by magnetic neutron scattering (de Gennes and Matricon, 1964), and the first experimental confirmation for the existence of vortices was indeed made by observing neutron diffraction from the VL in niobium (Cribier et al., 1964).

SANS studies of the VL can be considered as crystallography of a two-dimensional system of lines (vortices), providing information about the lattice structure and correlations, as well as the internal structure of the individual scatterers. While there are many similarities between the VL and the skyrmion lattice discussed in Sec. VIII, there are also important differences. Because of flux quantization, the vortex density depends linearly on the magnetic induction $B$. For a square VL, the magnitude of the fundamental (firstorder) scattering vector is given by

$$
q_{0}=2 \pi \sqrt{\frac{B}{\Phi_{0}}} .
$$

For a rhombic VL, i.e., oblique with equal side lengths but an arbitrary opening angle $60^{\circ}<\beta<90^{\circ}$, the scattering vector is 
$q=(\sin \beta)^{-1 / 2} q_{0}$, whereas a distorted triangular (hexagonal) lattice $\left(\beta<60^{\circ}\right)$ will have $q=(2 \tan \beta / 2)^{1 / 2} q_{0}$. From precise measurements of the scattering vector magnitude, it is therefore possible to determine the VL symmetry or to provide a relation between $B$ and the applied magnetic field $\mu_{0} H$.

The scattered intensity is directly related to the amplitude of the magnetic field modulation due to the vortices, quantified by the VL form factor. The latter is given by the Fourier transform of the two-dimensional field modulation $F(\mathbf{q})=\int B(\mathbf{r}) e^{i \mathbf{q} \cdot \mathbf{r}} d \mathbf{r}$. The form factor depends on the superconducting penetration depth $(\lambda)$ and coherence length $(\xi)$, and measuring $|F(q)|$ allows a determination of these characteristic length scales. ${ }^{4}$ This requires a measurement of the integrated scattered intensity, obtained by rotating the relevant VL diffraction peak through the Bragg condition in a rocking curve, as discussed in Sec. VIII.A.4 for the skyrmion lattice. Normalizing the integrated scattered intensity to the incident neutron flux one obtains the VL reflectivity

$$
R=\frac{2 \pi \gamma_{n}^{2} \lambda_{n}^{2} t}{16 \Phi_{0}^{2} q}|F(q)|^{2}
$$

where $t$ is the sample thickness, and $\lambda_{n}$ is the neutron wavelength (Kemoklidze, 1965; Christen et al., 1977). With modern SANS instruments it is possible to measure $|F(q)|$ as small as $0.1-0.2 \mathrm{mT}$, constituting tiny "ripples" on top of the average magnetic induction.

Early neutron scattering studies of the VL were performed using two-axis diffractometers (Cribier et al., 1964; Schelten, Ullmaier, and Schmatz, 1971; Schelten, Lippmann, and Ullmaier, 1974). Such measurements were challenging due to the long VL periodicity $\sim 2 \pi / q_{0}$, exceeding tens of nanometers even for the largest available magnetic fields and leading to scattering angles $<1^{\circ}$. This situation was improved using a double crystal geometry to separate the scattering due to the VL from the direct (undiffracted) beam (Christen et al., 1980, 1985). Still, only a few such studies were performed, mostly on niobium where the small $\lambda$ leads to scattering intensities in excess of $10 \%$ of the incident beam. The development of dedicated SANS instruments at cold neutron sources changed this situation dramatically. By providing a higher incident flux and allowing the entire scattering plane to be imaged using a two-dimensional position-sensitive detector (Fig. 36), these have made SANS an ideal tool for VL studies.

The emphasis in the following reflects the most studied problems and materials and current directions for SANS VL studies, but is not a comprehensive treatise. Shorter and more focused reviews are available elsewhere (Eskildsen, 2011; Eskildsen, Forgan, and Kawano-Furukawa, 2011).

\section{B. Vortices as probes of superconducting materials}

Information about the nature of the superconducting state in the host material can be obtained from the VL structure as well as the field and temperature dependence of the scattered intensity.

\footnotetext{
${ }^{4}$ To avoid confusion with the London penetration depth $\lambda$, the neutron wavelength is denoted as $\lambda_{n}$ throughout this section.
}
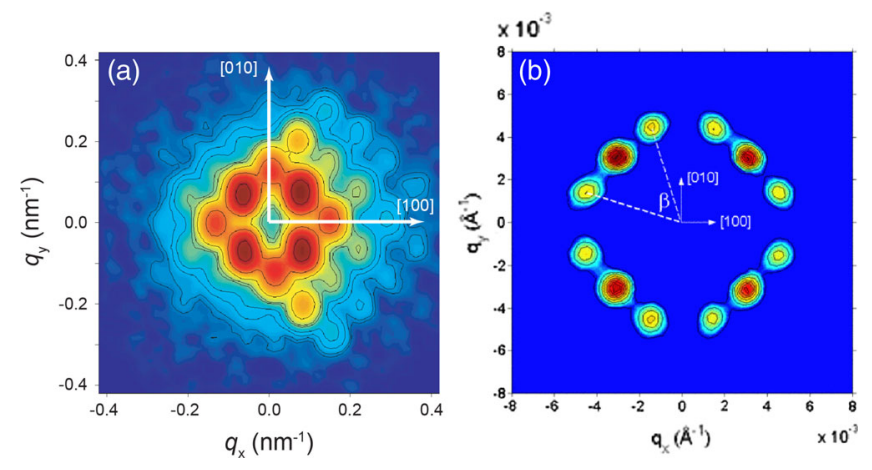

FIG. 36. SANS diffraction patterns showing (a) a square VL in $\mathrm{LuNi}_{2} \mathrm{~B}_{2} \mathrm{C}$ (Densmore et al., 2009) and (b) a rhombic VL in $\mathrm{YNi}_{2} \mathrm{~B}_{2} \mathrm{C}$ (Dewhurst, Levett, and McK. Paul, 2005).

\section{VL symmetry and orientation}

The equilibrium VL configuration is determined by the vortex-vortex interaction and in the ideal isotropic case will have a triangular symmetry (Kleiner, Autler, and Roth, 1964; Matricon, 1964). However, the free energy difference between the triangular and square symmetries is small (2\%), rendering the VL sensitive to an anisotropy of the screening current plane perpendicular to the applied field. Actual superconducting materials possess a hierarchy of anisotropies that will influence the VL symmetry and/or orientation relative to the crystalline axes. This often leads to degenerate VL orientations relative to the crystalline host and SANS diffraction patterns that include scattering from two or more domain orientations as seen in Figs. 36(b), 41(a), and 44(c) and 44(d).

The simplest example of a nontriangular VL is found in tetragonal superconductors with a fourfold basal plane anisotropy and the applied field parallel to the $\mathbf{c}$ axis. With increasing vortex density the contribution of the anisotropy to the free energy becomes dominant, leading to a sequence of VL transitions. A field-driven VL transition from a triangular to hexagonal symmetry was first observed in the borocarbide magnetic superconductor $\mathrm{ErNi}_{2} \mathrm{~B}_{2} \mathrm{C}$ (Yaron et al., 1996; Eskildsen et al., 1997; Yethiraj et al., 1997; Paul et al., 1998). Since then, similar transitions have been observed in a range of materials, including $\mathrm{V}_{3} \mathrm{Si}$ (Yethiraj et al., 1999), the high-temperature cuprate $\mathrm{YBa}_{2} \mathrm{Cu}_{3} \mathrm{O}_{7}$ (Brown et al., 2004), and the heavy-fermion $\mathrm{CeCoIn}_{5}$ (DeBeer-Schmitt et al., 2006). The triangular-to-square transition is so ubiquitous that it is more noteworthy when it is absent in a superconductor with a fourfold basal plane anisotropy (Riseman et al., 1998; Gilardi et al., 2004; Kawano-Furukawa et al., 2011; Morisaki-Ishii et al., 2014). More recently it was also observed for the skyrmion lattice (Karube et al., 2016) underscoring the similarities between the VL and the SkL.

Theoretically, nonlocal corrections to the London model describe a VL symmetry evolution driven by a Fermi surface anisotropy (Kogan et al., 1997). The transition is characterized by two critical fields as shown in Fig. 37(a). Here a rhombic (distorted triangular) VL [Fig. 36(b)] undergoes a first-order reorientation transition at $H_{1}$, followed by a continuous increase of the opening angle toward a second-order transition at $H_{2}$ above which a square VL [Fig. 36(a)] is observed. Figure 37(b) shows results of a detailed study of the VL in 
(a)

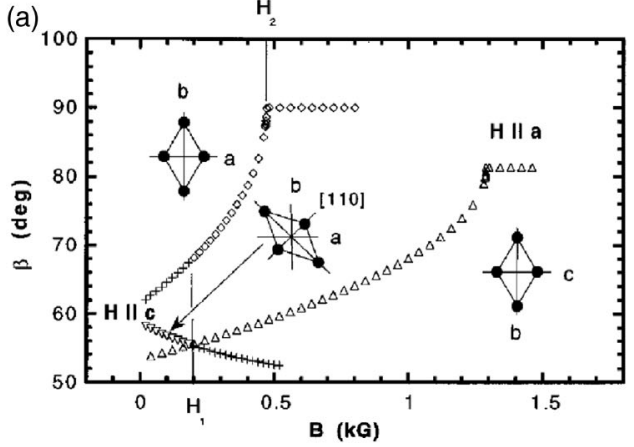

(b)

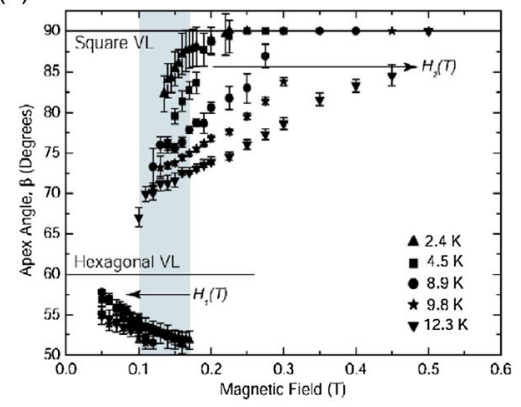

(c)

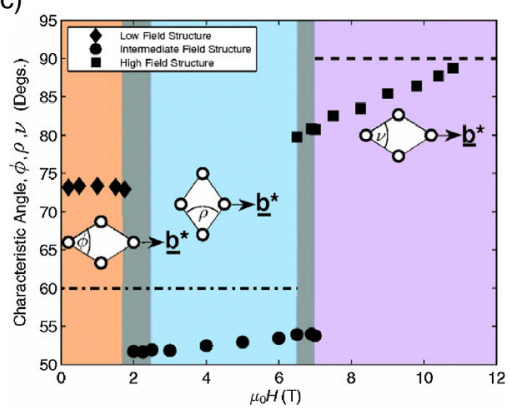

FIG. 37. Density driven VL symmetry transition for $\mathbf{H} \| \mathbf{c}$ in superconductors with a fourfold basal plane anisotropy. (a) Equilibrium VL opening angle predicted from nonlocal corrections to the London model (Kogan et al., 1997). (b) Measured opening angle in $\mathrm{YNi}_{2} \mathrm{~B}_{2} \mathrm{C}$ for different temperatures (Dewhurst, Levett, and McK. Paul, 2005). (c) Field dependence of the opening angle (2 K) for the VL structures in $\mathrm{YBa}_{2} \mathrm{Cu}_{3} \mathrm{O}_{7}$ (White et al., 2011). Gray shading in (b) and (c) indicate where first-order VL reorientation transitions are observed.

$\mathrm{YNi}_{2} \mathrm{~B}_{2} \mathrm{C}$, where coexisting low- and high-field rhombic phases are observed near the first-order reorientation transition (Dewhurst, Levett, and McK. Paul, 2005). At higher temperatures the gap between $H_{1}$ and $H_{2}$ grows, consistent with increased thermal fluctuations close to the upper critical field (Gurevich and Kogan, 2001). Separate studies of doped samples showed that $\mathrm{H}_{2}$ depends sensitively on the nonlocality range (Gammel et al., 1999).

An additional contribution to a fourfold anisotropy may also come from the superconducting gap, e.g., in materials with non-s-wave pairing symmetry (Franz, Affleck, and Amin, 1997; Agterberg, 1998; Ichioka, Hasegawa, and Machida, 1999). In $\mathrm{YBa}_{2} \mathrm{Cu}_{3} \mathrm{O}_{7}$ early SANS studies showed diffraction patterns with a fourfold structure due to pinning to twin boundaries (Forgan et al., 1990; Yethiraj et al., 1993b; Keimer et al., 1994). More recent measurements on detwinned samples revealed a complex evolution of the VL symmetry and orientation, Fig. 37(c) (White et al., 2008, 2009, 2011). The presence of two, first-order reorientation transitions may naively be attributed to the Fermi surface and gap anisotropies separately. However, it has proven difficult to deconvolute these two contributions to the anisotropy (Leos et al., 2015) and a definitive understanding of the VL symmetry in such cases is still lacking.

Compared to the previously discussed materials, the conventional superconductor niobium exhibits a surprisingly rich VL phase diagram. Soon after the first VL observation it was recognized that different configurations could be expected for fields applied along different crystalline directions in this cubic material (Takanaka, 1971, 1973a, 1973b). This was confirmed in pioneering experiments carried out with fields along twofold, fourfold, and sixfold symmetric axes (Schelten, Lippmann, and Ullmaier, 1974; Christen et al., 1980). More recent studies found additional VL phases for fields along the fourfold [100] axis which all break some crystal symmetry, Fig. 38(a) (Laver et al., 2006, 2009). The transition between the different VL configurations is attributed to the combination of a pronounced Fermi surface anisotropy and the significant vortex core overlap in niobium due to the low ratio between the penetration depth and coherence length $\kappa=\lambda / \xi$ (Laver et al., 2009; Adachi et al., 2011). The low field square VL shown in Fig. 38(a) is considered to be a property of the intermediate mixed state (IMS) discussed later (Mühlbauer, Pfleiderer et al., 2009). Finally, the square and
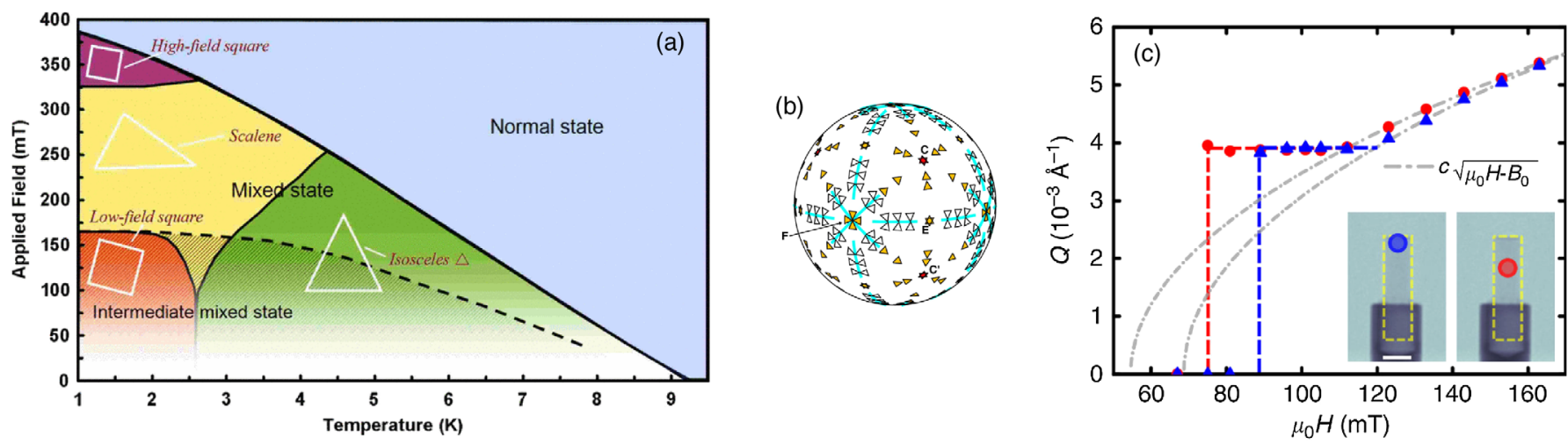

FIG. 38. VL phases in niobium. (a) Phase diagram for applied fields along the fourfold [001] axis (Laver et al., 2009). (b) VL structures observed as a function of field direction (Laver and Forgan, 2010). Isosceles half-unit cells are indicated by filled triangles, and equilateral triangles are colored in red. Thick lines show sudden changes in the VL orientation. (c) Intermediate mixed phase indicated by a constant VL scattering vector below $120 \mathrm{mT}$ at two different positions within the same crystal (Reimann et al., 2015). Gray lines correspond to a vortex density $\propto H$, Eq. (64). 
scalene VL phases are not oriented with a nearest-neighbor direction along a crystalline high-symmetry direction, showing the presence of higher-order anisotropy terms in the free energy. The likely existence of VL phases that spontaneously break the underlying crystal symmetry can also be understood as a purely topological effect. Application of the so-called "hairy ball" theorem to the case of niobium, as the field is rotated between high-symmetry directions, shows that VL discontinuities must exist, as illustrated in Fig. 38(b) (Laver and Forgan, 2010). Compared to individual skyrmions, each characterized by a nonzero winding number, the topology here governs the behavior of the collective VL. That said, the hairy ball theorem also applies to the SkL.

The low $\kappa$ for niobium leads to a local minimum in the vortex-vortex interaction potential, giving rise to an IMS at low fields where flux free Meissner regions and VL regions coexist (Aston, Dubeck, and Rothwarf, 1971; Christen et al., 1977). In the IMS, the vortex separation, and hence $q_{0}$, is independent of the applied field, as illustrated in Fig. 38(c). With increasing $H$, the VL regions grow at the expense of the Meissner ones until they fill the entire sample, after which $q$ follows the $\sqrt{H}$ behavior given by Eq. (64). Upon cooling through the superconducting transition, vortex clusters with a constant periodicity were found to coexist with a regular Abrikosov (or Shubnikov) VL phase, before the latter vanishes at low temperature to produce the IMS state (Pautrat and Brûlet, 2014). Because of its inherent inhomogeneity, the IMS may be used as a model system for domain nucleation and morphology. Recently, this was studied using spatially resolved SANS combined with neutron grating interferometry to obtain detailed spatial information about the structure of the IMS domains and how they expand, Fig. 38(c) (Reimann et al., 2015).

\section{Field and temperature dependence of the VL form factor}

The simplest model for the VL form factor is provided by the local London theory. This relates the supercurrent density to the magnetic vector potential at the same point via the penetration depth, extended by a Gaussian cutoff to take into account the finite coherence length (Eskildsen, Forgan, and Kawano-Furukawa, 2011)

$$
F(q)=\frac{B}{1+q^{2} \lambda^{2}} e^{-c q^{2} \xi^{2}} .
$$

Here $c$ is a constant of order unity (Yaouanc, Dalmas de Réotier, and Brandt, 1997). In most cases $q \lambda \ll 1$, and Eq. (66) predicts a form factor that decreases exponentially with increasing as with $q^{2} \propto B$. This is due to an increased core overlap resulting in a rapid reduction of the field modulation, even as the number of vortices increases. More rigorous models based on the Ginzburg-Landau theory (Clem, 1975; Hao et al., 1991) as well as numerical calculations carried out within the Eilenberger formalism (Ichioka, Hasegawa, and Machida, 1999) confirm the monotonic decrease of $F(q)$ while yielding deviations from a pure exponential behavior. For strongly type-II superconductors $(\lambda / \xi \gg 1)$ the extended London and Ginzburg-Landau form factors agree at low fields. Measuring $F(q)$ at fields that are simultaneously small, but still large enough to satisfy $q \lambda \gg 1$, will therefore allow an extrapolation to $q=0$ and an estimate of the penetration depth which is unaffected by vortex core effects.

Figure 39(a) shows the measured form factor for the primary $\mathrm{VL}$ reflection in $\mathrm{LuNi}_{2} \mathrm{~B}_{2} \mathrm{C}$ (Densmore et al., 2009). For this material $|F(q)|$ is best described by the London model (solid line) rather than the more sophisticated models (dashed lines). In addition to the determination of $\lambda$ from the $q=0$ extrapolation as discussed, an estimate of the coherence length may be obtained from the slope of $\ln |F(q)|$ vs $\mu_{0} H$. Here a $c=1 / 2$ is commonly used in Eq. (66), which is found to yield reasonable values for $\xi$. This is further justified by a quantitative comparison to numerical results (Ichioka, Hasegawa, and Machida, 1999), which suggests an appropriate value of 0.44 at low temperatures (Bowell, 2008; Eskildsen, Forgan, and Kawano-Furukawa, 2011). In cases where more than the primary VL reflection can be measured, a more detailed analysis of the form factor is possible, including comparison to complex models as for $\mathrm{Sr}_{2} \mathrm{RuO}_{4}$ (Kealey et al., 2000). In materials such as $\mathrm{LuNi}_{2} \mathrm{~B}_{2} \mathrm{C}$ where it is possible to measure a large number of higher-order $\mathrm{VL}$ reflections as
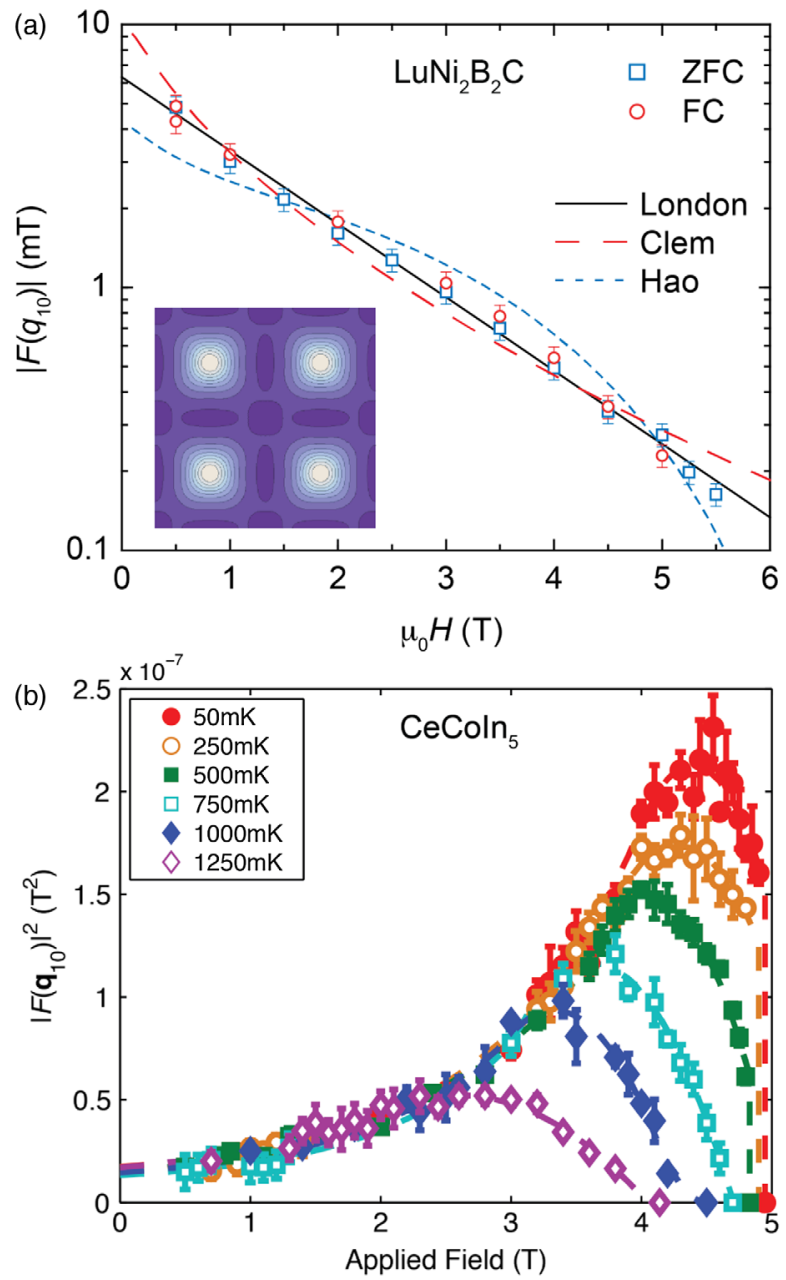

FIG. 39. Field dependence of the VL form factor in (a) $\mathrm{LuNi}_{2} \mathrm{~B}_{2} \mathrm{C}$ (Densmore et al., 2009) and (b) $\mathrm{CeCoIn}_{5}$ (White et al., 2010). The inset in (a) shows the real-space field reconstruction. 
shown in Fig. 36(a), the real-space field distribution may be obtained by $B(\mathbf{r})=\sum F\left(\mathbf{q}_{h k}\right) e^{i \mathbf{q}_{h k} \cdot \mathbf{r}}$ and shown in the inset to Fig. 39(a).

A deviation from the conventional field dependence occurs in materials with a strong coupling between the applied field and the quasiparticle spins. This was first reported for $\mathrm{TmNi}_{2} \mathrm{~B}_{2} \mathrm{C}$ (DeBeer-Schmitt et al., 2007) and later observed in a more extreme form in $\mathrm{CeCoIn}_{5}$ (Bianchi et al., 2008; White et al., 2010). Results for the latter are shown in Fig. 39 (b). At all temperatures, the form factor initially increases before reaching a maximum and then decreasing on approaching the upper critical field $H_{\mathrm{c} 2}$. Below $500 \mathrm{mK} F(q)$ remains finite up to $H_{\mathrm{c} 2}$ above which it vanishes abruptly, consistent with the first-order nature of the superconducting transition in $\mathrm{CeCoIn}_{5}$ at low temperatures. The unusual field dependence of $F(q)$ is due to a strong exchange coupling of the conduction electrons and the $\mathrm{TmNi}_{2} \mathrm{~B}_{2} \mathrm{C}$ or CeCoIn sublattice moments (Ichioka et al., 2007). The enhanced Pauli paramagnetic effects lead to a polarization of the unpaired quasiparticle spins in the vortex cores and a spatially varying paramagnetic moment, providing an additional contribution to the field variation in the mixed state. Approaching $H_{\mathrm{c} 2}$, the paramagnetic depairing causes the vortex cores to expand, making them more isotropic. For $\mathbf{H} \| \mathbf{c}$ this suppresses the fourfold anisotropy in the screening current plane, leading to a reentrance of the square VL phase (Eskildsen et al., 1998; Bianchi et al., 2008; Das, Densmore et al., 2012).

The temperature dependence of the VL form factor reflects the structure of the superconducting gap $\Delta$. Specifically, $F(q)$ is proportional to the superfluid density $\rho_{s} \propto \lambda^{-2}$ given by

$\rho_{s}(t)=1-\frac{1}{4 \pi t} \int_{0}^{2 \pi} \int_{0}^{\infty} \cosh ^{-2}\left(\frac{\sqrt{\varepsilon^{2}+\Delta^{2}(t, \phi)}}{2 t}\right) d \phi d \varepsilon$

where $t=T / T_{C}$ is the normalized temperature (Prozorov and Giannetta, 2006; Eskildsen, Forgan, and Kawano-Furukawa, 2011). Here $T_{C}$ is the superconducting critical temperature and the gap is in units of $k_{B} T_{C}$. The latter can be separated into temperature- and momentum-dependent parts $\Delta(t, \phi)=$ $\Delta_{0}(t) \Delta_{k}(\phi)$. The $T$ dependence is given by the weak coupling expression

$$
\Delta_{0}(t)=\Delta_{0}(0) \tanh \left(\frac{\pi}{\Delta_{0}(0)} \sqrt{\frac{1}{t}-1}\right)
$$

where $\Delta_{0}(0)$ is the zero-temperature amplitude (Gross et al., 1986). With increasing temperature $F(q)$ decreases due to thermal excitation of quasiparticles across the gap and eventually vanishes at $T_{C}$. For $t \lesssim 1 / 3$ the temperature dependence is dominated by the lowest values of the gap. If $\Delta_{k}(\phi)$ is large over the whole Fermi surface, few quasiparticles are excited and the scattered intensity $I \propto|F(q)|^{2}$ is nearly constant, Fig. 40(b). In contrast, for a nodal gap the intensity will vary with temperature even as $t \rightarrow 0$, in a manner that depends on the location and dispersion of the nodes. The coherence length changes little for $t \ll 1$ and is often ignored in the temperature dependence, although it
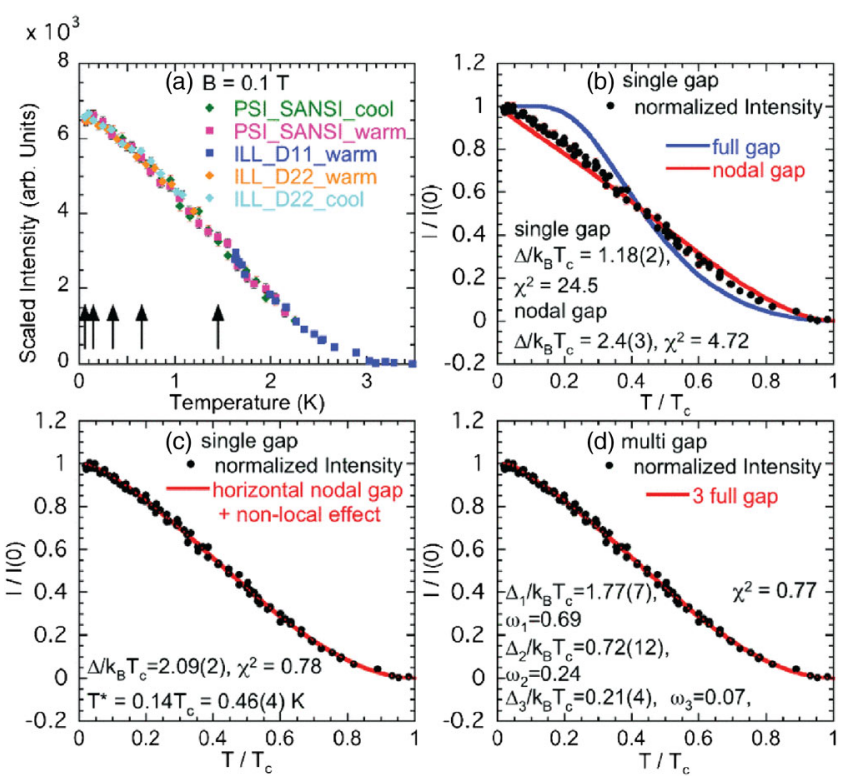

FIG. 40. Temperature dependence of the VL peak intensity in $\mathrm{KFe}_{2} \mathrm{As}_{2}$ (Kawano-Furukawa et al., 2011). (b)-(d) Different fits to the original data in (a). The difference between a full and nodal gap is illustrated in (b).

would be straightforward to include since within the BardeenCooper-Schrieffer theory $\xi \propto \Delta_{0}(t)^{-1}$.

Figure 40(a) shows the VL scattered intensity as a function of temperature in the iron-based superconductor $\mathrm{KFe}_{2} \mathrm{As}_{2}$ (Kawano-Furukawa et al., 2011). Here the intensity has a strong $T$ dependence down to the lowest measured temperatures, indicating a range of gaps that extend down to zero or near-zero values. The best fit to the data is from a single nodal gap coupled with nonlocal effects shown in Fig. 40(c) or to a three-gap model with a small lowest $\Delta$, Fig. 40(d) (KawanoFurukawa et al., 2011). The nonlocal corrections become important for nodal gaps where the London assumption of a vanishing ratio of $\xi / \lambda$ breaks down. From the temperature dependence alone it is not possible to discriminate between the two scenarios, but, as discussed later, there is strong evidence for multiband superconductivity in $\mathrm{KFe}_{2} \mathrm{As}_{2}$. Similar studies were recently used to provide information about the nodal structure of the unconventional superconductor $\mathrm{UPt}_{3}$ (Gannon et al., 2015). Here measurements with different directions of the applied field were compared to models of both the location and dispersion of the gap nodes.

\section{Complex order parameters, multigap superconductivity, and Pauli limiting}

The ability to probe within the mixed (vortex) state allows VL SANS measurements to extract subtle features about the superconducting state.

With three distinct superconducting phases (denoted A, B, and $\mathrm{C}$ ) the heavy-fermion $\mathrm{UPt}_{3}$ is a paradigm for unconventional superconductivity (Joynt and Taillefer, 2002). This material has a hexagonal crystal structure but, within the $E_{2 u}$ model, a nodal gap with a fourfold symmetry in the superconducting $\mathrm{A}$ and $\mathrm{C}$ phases. The lower rotational symmetry of the gap gives rise to a rotation of the triangular 
(a)

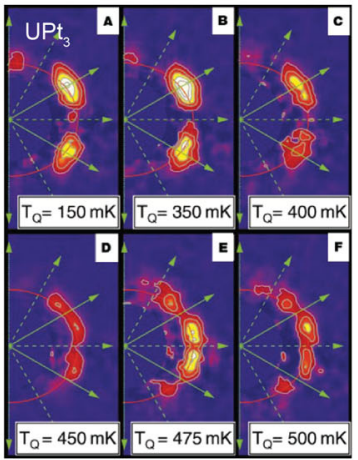

(b)

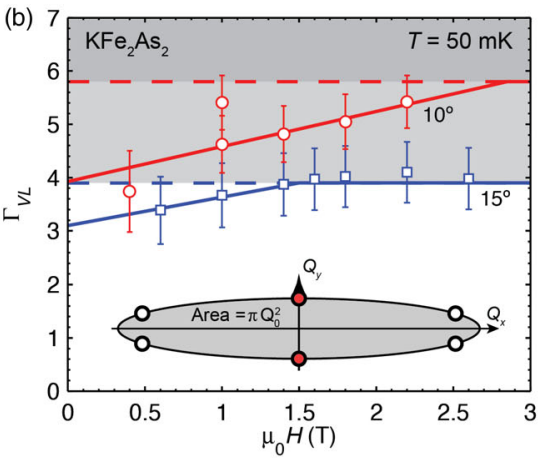

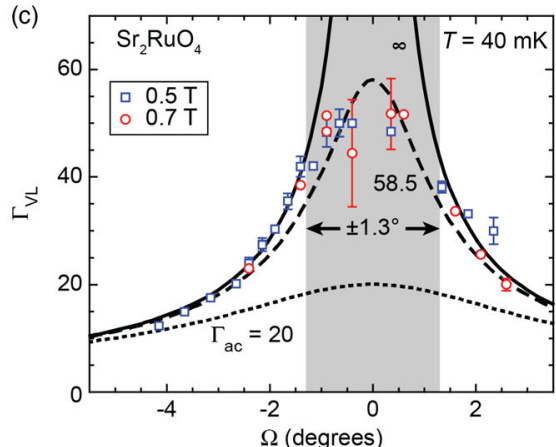

FIG. 41. Probing complex superconducting states. (a) Diffraction patterns in $\mathrm{UPt}_{3}$ for $\mathbf{H} \| \mathbf{c}$, "grown" at $190 \mathrm{mT}$ and $T_{Q}$ and quenched to the measurement temperature (100-150 mK) (Huxley et al., 2000). (b) VL anisotropy as a function of magnetic fields applied close to the basal plane in $\mathrm{KFe}_{2} \mathrm{As}_{2}$ (Kuhn et al., 2016). The inset shows a schematic of VL Bragg reflections lying on an ellipse with major-tominor axis ratio $\Gamma_{\mathrm{VL}}$. (c) $\mathrm{VL}$ anisotropy vs field angle in $\mathrm{Sr}_{2} \mathrm{RuO}_{4}$ consistent with a superconducting anisotropy $\Gamma_{a c}=58.5 \pm 2.3$ (Rastovski, Dewhurst et al., 2013).

VL, Fig. 41(a) (Huxley et al., 2000). These measurements focused on the A phase which exists at low fields in a narrow temperature range just below $T_{C}$. SANS studies of $\mathrm{UPt}_{3}$ are challenging due to the large penetration depth, and measurements within the A phase itself are not feasible due to the further loss of intensity when approaching $T_{C}$. Instead, a quenching technique was applied, where an equilibrium $\mathrm{VL}$ is obtained by a field oscillation at $T_{Q}$ and then cooled to the lower measurement temperature. For $\mathrm{UPt}_{3}$ thermal effects are sufficiently weak that the VL remains in the configuration obtained at the quench temperature $T_{Q}$. The diffraction patterns in Fig. 41(a) show how triangular VL domains, oriented with Bragg peaks along the crystalline a axis at low temperature, rotate by $\pm 15^{\circ}$ as the A phase is approached. This allowed a determination of the A-phase nodal structure, with antinodes along the $\mathbf{a}$ and $\mathbf{a}^{*}$ crystalline axes (Huxley et al., 2000; Champel and Mineev, 2001).

Rotating the applied field away from the principal axis in a uniaxial superconductor introduces a twofold anisotropy, causing a distortion of the VL and Bragg peaks that lie on an ellipse rather than a circle, as shown in the inset to Fig. 41(b) (Campbell, Doria, and Kogan, 1988; Keimer et al., 1993; Yethiraj et al., 1993a; Gammel et al., 1994; Das et al., 2012). The VL anisotropy $\Gamma_{\mathrm{VL}}$ is given by the major-to-minor axis ratio, and due to flux quantization it is sufficient to measure the location of the VL Bragg peaks along the minor ellipse axis (Rastovski, Dewhurst et al., 2013; Kuhn et al., 2017). For fields parallel to the basal plane $\Gamma_{\mathrm{VL}}=\Gamma_{a c}$. Here $\Gamma_{a c}$ mainly reflects the anisotropy of the Fermi velocity in the $a c$ plane, and in general one expects $\Gamma_{a c}=\lambda_{c} / \lambda_{a b}$ (KawanoFurukawa et al., 2013). Even in cases where measurements with an exact in-plane field are not possible, $\Gamma_{a c}$ can be obtained by extrapolation. The ability to measure $\Gamma_{a c}$ is especially useful in materials where this is not equal to the upper critical field anisotropy because $H_{\mathrm{c} 2}$ is Pauli limited along one or more crystalline directions.

In materials where the superconductivity resides on multiple Fermi surface sheets with different anisotropy one often finds a field and/or temperature dependence of $\Gamma_{a c}$. This was first reported for $\mathrm{MgB}_{2}$ and attributed to a more rapid suppression of the superconductivity on the Fermi surface sheets with the smaller gap (Cubitt et al., 2003). Figure 41(b) shows measurements of $\Gamma_{\mathrm{VL}}$ in $\mathrm{KFe}_{2} \mathrm{As}_{2}$ for two different angles close to the basal plane (Kuhn et al., 2016). This shows a clear field dependence, indicative of multigap superconductivity in this material. Moreover, $\Gamma_{\mathrm{VL}}$ exceeds the upper critical field anisotropy of $\sim 3.3$ and thus shows Pauli limiting for in-plane fields.

In highly anisotropic superconductors spin-flip scattering, due to the transverse VL field modulation, can greatly exceed the non-spin-flip scattering usually used to image the VL (Thiemann, Radovic, and Kogan, 1989; Kealey et al., 2001; Amano et al., 2014; Kuhn et al., 2016). Since the spin-flip scattering vanishes for fields within the basal plane, this also allows a precise in situ alignment of the magnetic field. The spin-flip scattering was utilized in SANS studies of the anisotropy in $\mathrm{Sr}_{2} \mathrm{RuO}_{4}$ which would otherwise not have been possible, Fig. 41(c) (Rastovski, Dewhurst et al., 2013; Nakai and Machida, 2015). This found $\Gamma_{a c} \sim 60$, greatly exceeding that of the upper critical field $\left(\Gamma_{H_{c 2}} \sim 20\right)$ indicating Pauli limiting similar to the situation in $\mathrm{KFe}_{2} \mathrm{As}_{2}$. This raises questions concerning the order parameter in $\mathrm{Sr}_{2} \mathrm{RuO}_{4}$ which is considered to be a $p$-wave superconductor with equal-spin pairing where Pauli limiting is not expected to occur (Maeno et al., 2012).

\section{Vortex matter studies}

While the repulsive vortex-vortex interaction favors the formation of a well-ordered VL, thermal effects and/or pinning to imperfections can lead to disordering. The balance between these competing factors determines both the structural and dynamic properties of vortex matter which is interesting in its own right and as a model system for the behavior of soft matter in general.

\section{Structural properties and correlations}

Obtaining an ordered VL with a well-defined diffraction pattern often requires "annealing" to remove disorder that may have been frozen in during a field cooling procedure. In materials with weak pinning this can be achieved by applying a transport current (Yaron et al., 1994, 1995; Pautrat et al., 
2005) or by a damped, small-amplitude ac field to "shake" the vortices into their equilibrium positions (Levett, Dewhurst, and McK. Paul, 2002). In contrast, it is often not possible to achieve an ordered VL in superconductors with strong pinning, and a shaking can even lead to a further disordering of the VL. This is seen in members of the iron-based superconductors where a ring of scattering from a VL powder is observed (Eskildsen et al., 2009; Inosov et al., 2010a, 2010b).

For a well-ordered VL the correlation lengths are determined from SANS, being inversely proportional to the width of the Bragg reflections in reciprocal space (Yaron et al., 1995). A measure of the longitudinal correlation length (vortex "straightness") is obtained from rocking curves (RC) of the scattered intensity as the VL peaks are rotated through the Bragg condition, shown in Figs. 43(b) and 43(c). While the poor resolution in the detector plane makes it difficult to determine the positional order directly, this can be estimated from the RC width, as the longitudinal, translational, and orientational correlation lengths are related by the VL elastic constants (Brandt, 1995).

A fundamental question is the exact structural characteristics of a dislocation free VL. For any amount of disorder the positional order will decay over some characteristic length scale, where the vortex displacement becomes of the order of the VL spacing. In the so-called Bragg glass model, where the VL is treated as an elastic manifold, the positional order has a weak, power-law decay, giving rise to rocking curves with a decreasing amplitude but no broadening (Cubitt et al., 1993; Klein et al., 2001). This is supported by the measurements in Fig. 42(a) showing a decay positional correlation, i.e., of the integrated intensity multiplied by $q /|F(q)|^{2}$, which, from Eq. (65), is expected to be constant. More recent SANS measurements used a transverse magnetic field, where the $\mathrm{RC}$ width probes the positional order, coupled with a reverse Monte Carlo analysis of the data (Laver et al., 2008). This found some evidence for a Bragg glass at large length scales, but also a fracturing shown in Fig. 42(b) which leads to RC broadening. Subsequent studies using a time-of-flight approach to SANS yielded consistent results (Pautrat et al., 2012).
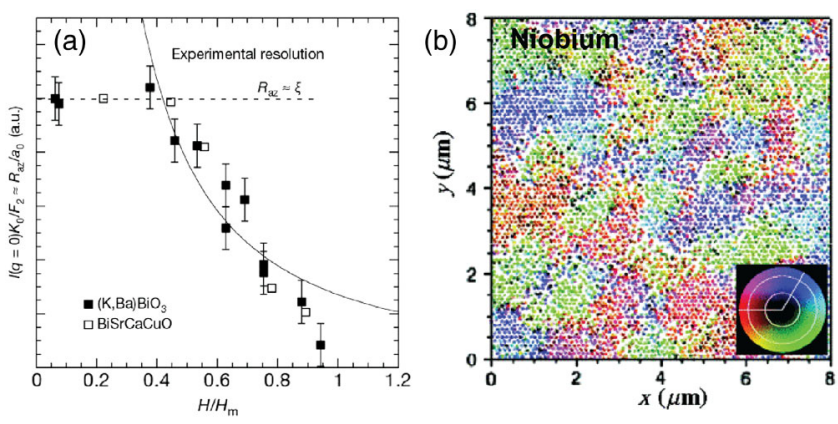

FIG. 42. Structural properties of the VL. (a) Positional correlation $\left(R_{a z}\right)$ length for $(\mathrm{K}, \mathrm{Ba}) \mathrm{BiO}_{3}$ and $\mathrm{BiSrCaCuO}$ (Klein et al., 2001). The horizontal dotted line is where $R_{a z}$ becomes larger than the experimental resolution. (b) VL positions in a reverse Monte Carlo ensemble based on SANS measurements in niobium (Laver et al., 2008). Colors denote the magnitude (lightness) and direction (hue) of the in-plane displacement.

\section{Relating structural and dynamic properties}

Vortex matter exhibits a rich and complex dynamic behavior (Blatter et al., 1994; Brandt, 1995). This includes an increased depinning critical current in a region below the upper critical field, known as the peak effect (PE), and VL melting, where SANS can provide complementary structural information. The first report of VL melting observed by SANS was in the hightemperature superconductor $\mathrm{Bi}_{2.15} \mathrm{Sr}_{1.95} \mathrm{CaCuO}_{8+x}$, where an abruptly vanishing intensity was observed at temperatures much less than $T_{C}$ (Cubitt et al., 1993). Reports of melting, based on the observation of a VL disordering, were also made for niobium where effects of thermal fluctuations are expected to be weak. However, subsequent measurements on ultrapure samples contradicted these findings, revealing an ordered $\mathrm{VL}$ in very close proximity to $H_{\mathrm{c} 2}$ (Forgan et al., 2002; Bowell et al., 2010). The difference may be attributed to differences in sample quality, demonstrating the challenge in eliminating extrinsic effects.

In the case of the PE, the increase in the critical current is commonly associated with a softening of the VL and an ensuing order-disorder transition, which better allows it to conform with the pinning landscape (Gammel et al., 1998; Joumard et al., 1999). As already discussed, energy barriers may trap the VL in disordered metastable configurations when cooling from the normal state in a constant field. In addition to a transport current or an ac field, an ordering of the VL can in some cases be achieved by a simple thermal cycling (Daniilidis et al., 2007; Marziali Bermudez et al., 2017). Conversely, the application of a field oscillation will introduce an edge contamination of the VL at surfaces where vortices enter and leave the sample (Hanson et al., 2011). While associating the PE with a bulk order-disorder transition is widely accepted, this is not universal. This is due to some observations of an ordered VL above the PE (Pautrat et al., 2007, 2009) and studies which indicate that this is caused by surface rather than bulk pinning (Pautrat et al., 2012). Here one should note that few SANS measurements are performed under the same exact conditions (transport currents, ac fields) as those used to determine the PE. This emphasizes the need for simultaneous transport measurements when drawing connections between the structural and dynamic properties of vortex matter. Recent measurements combined SANS with in situ linear ac susceptibility measurements as shown in Fig. 43 (Marziali Bermudez et al., 2015). These studies explored the behavior in the complex transitional region adjacent to the PE, where pinning can either decrease or increase by the application of transport currents or ac magnetic fields.

\section{Ordered, nonequilibrium VL phases}

A final example of complex vortex matter behavior is the recent discovery of well-ordered, metastable VL phases (Das, Rastovski et al., 2012). In $\mathrm{MgB}_{2}$ the triangular VL undergoes a $30^{\circ}$ continuous rotation transition, similar to the one observed in $\mathrm{UPt}_{3}$ and shown in Fig. 41(a), but here attributed to a Fermi surface anisotropy coupled with the two-gap nature of the superconducting state (Cubitt et al., 2003; Hirano et al., 2013). The diffraction patterns in Fig. 44 show examples of two of the phases in this material: a VL aligned with Bragg 

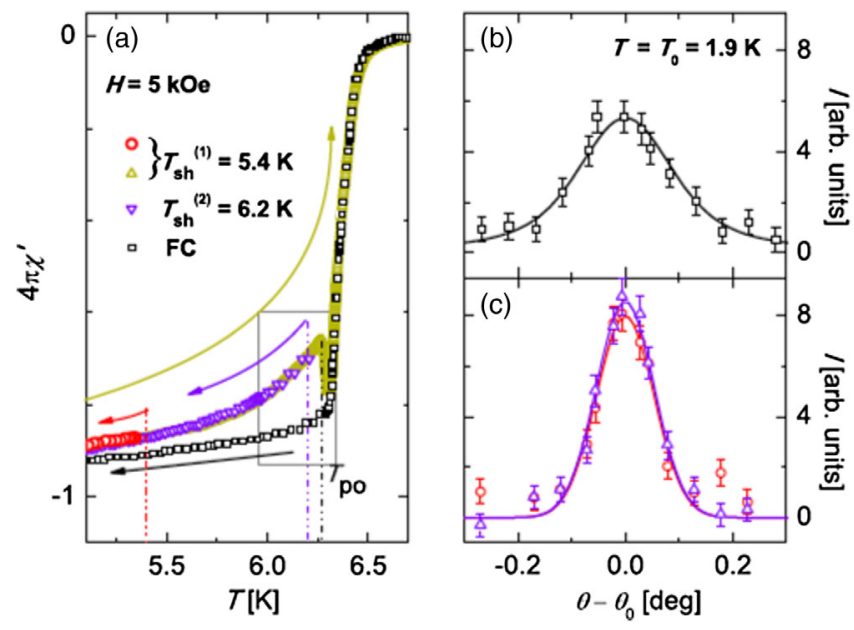

FIG. 43. Peak effect in $\mathrm{NbSe}_{2}$ (Marziali Bermudez et al., 2015). (a) Linear ac susceptibility in the vicinity of the PE for different field histories. (b) Rocking curve after a field cooling procedure. (c) RCs after "shaking" the VL at two different temperatures below the PE and with widths that are resolution limited.

peaks along the crystalline $a$ axis (F phase), and rotated away from $a$ resulting in two degenerate domain orientations (L phase). Cooling or warming across the equilibrium F-L phase transition leaves the VL in robust metastable states, and an external perturbation such as a field oscillation is required to drive it to the ground state, as shown in Fig. 44. The metastability is notably not due to pinning (Rastovski, Schlesinger et al., 2013), but represents a novel collective vortex phenomenon most likely stabilized by VL domain boundaries. More recently, a similar phenomenon was observed for skyrmion lattices where different phases could be stabilized depending on the temperature and field history (Makino et al., 2017). Looking ahead, the $\mathrm{VL}$ in $\mathrm{MgB}_{2}$ could possibly be used as a model system for nonequilibrium phase transitions.

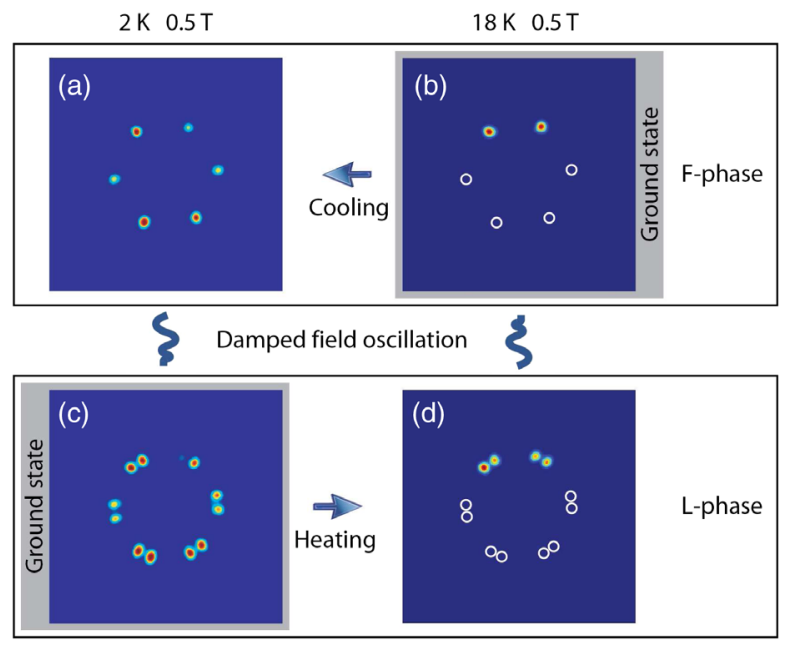

FIG. 44. Metastable VL phases in $\mathrm{MgB}_{2}$ (Das, Rastovski et al., 2012). (a), (d) Cooling or heating across the F-L phase transition leaves the VL in a metastable configuration. (b), (c) The ground state VL is obtained by applying a damped field oscillation.

\section{CONCLUDING REMARKS}

We have presented recent applications of magnetic SANS to study a wide range of magnetic materials, such as soft magnetic Fe-based nanocomposites, hard magnetic Nd-Fe-Bbased permanent magnets, magnetic steels, nanoparticles and ferrofluids, magnetic oxides and complex alloys, skyrmion lattices, spiral magnetic structures, and vortex lattices in typeII superconductors. The final paragraphs are devoted to a brief discussion of future developments, related to scientific grand challenges, theoretical and simulation work, as well as to SANS instrumentation.

While for bulk ferromagnets the theoretical framework of magnetic SANS has recently been developed (Michels and Weissmüller, 2008; Michels, 2014; Metlov and Michels, 2015, 2016; Mettus and Michels, 2015; Michels et al., 2016), the theoretical description of magnetic SANS for magnetic nanoparticles in a nonmagnetic matrix is still in its infancy. This comprises the description of spin arrangements in particles beyond the single-domain (supermoment) approximation and interparticle correlations in dense interacting particle systems. Future research will therefore concentrate on the analytical and/or numerical micromagnetic computation of the magnetic SANS cross section of a dispersion of magnetic nanoparticles in a nonmagnetic matrix - the classical prototypical sample microstructure in many magnetic SANS experiments. There is ample theoretical (Berger et al., 2008; Gatel et al., 2015; Vivas, Yanes, and Michels, 2017) as well as experimental (Disch et al., 2012; Günther et al., 2014; Krycka et al., 2014) evidence that nanosized magnetic particles are not homogeneously magnetized, and the question thus arises whether the standard expression for the cross section, Eq. (23), is still adequate to describe magnetic SANS. Intraparticle spin disorder may be due to the interplay between different magnetic interactions such as surface anisotropy and dipolar interaction, deviations from ellipsoidal particle geometry, or to the presence of crystal defects and antiphase boundaries (Nedelkoski et al., 2017). Heterogeneous particles offer an additional degree of freedom to tailor magnetic properties; for instance, in multiphase core or shell particles, the spin structure may show varying magnetic properties between phases (e.g., AFM core or FM shell). For nanoparticles, boundary conditions for the magnetization at internal and particle-matrix interfaces have to be taken into account, a task which, from the micromagnetic point of view, severely complicates the problem. Nucleation theory (Aharoni, 1996) may provide a guideline for attacking this problem.

For hard magnetic materials, magnetic SANS has allowed a novel perspective where a qualitative examination has been complemented by a semiquantitative analysis. Although fundamental topics are expected to be further explored, applied research will keep gaining momentum. SANS data suggested that the grain-boundary phase in sintered Nd-Fe-Bbased magnets is the main source of spin misalignment within the hard magnetic phase, and whose dimensions correlate with the defect size as observed by SANS. In fact, SANS is still expected to be the only technique to monitor the bulk of the (magnetic) microstructure, relating coercivity and defect dimensions in sintered magnets. Similarly, Nd-Fe-B nanocomposites will increasingly rely on SANS with the aim to 
simultaneously address composition, processing conditions, and magnetic properties. Future activities on the topic will embrace other engineering magnetic materials, likely with emphasis on Sm-Co compounds [possibly using Sm isotope (s) due to its large neutron absorption] in view of their importance for high-temperature applications combined with a microstructure that resembles the one of $\mathrm{Nd}-\mathrm{Fe}-\mathrm{B}$ counterparts; besides, the magnetic microstructure of ferrites, Alnico, or manganese compounds have not yet been studied using SANS.

SANS investigations on steels take advantage of a perfect correspondence between the size scale most relevant for the mechanical properties of advanced steels and the sensitivity range of SANS. The reported applications of magnetic SANS in the field of ferromagnetic steels indicate the need for an improved understanding of the magnetic properties of nanofeatures such as oxide nanoparticles or irradiation-induced solute atom clusters. This will be achieved by refining models of magnetism in complex systems at the nm scale and by using SANS to calibrate the models. The consideration of both magnetic and nuclear SANS is critical for the separation of magnetic and morphological aspects of the nanofeatures and may require magnetic-field-dependent SANS measurements.

Numerical micromagnetic computations can take into account the full nonlinearity of Brown's static equations of micromagnetics and, indeed, have provided fundamental insight into magnetic SANS. A particular advantage of micromagnetic simulations resides in their flexibility regarding microstructure variations (particle-size distribution, texture, magnetic materials parameters, etc.); it is also rather straightforward to "switch on" and "off" certain magnetic interactions in the simulations and to test in this way their impact on the neutron scattering. In view of the continuously increasing power of modern computers, further understanding of magnetic neutron scattering may also be expected from the development of efficient micromagnetic algorithms, so that true macroscopic samples, with dimensions of the order of several microns, can be simulated. In general, we believe that the combination of experimental scattering data with largescale numerical computations will become more and more important. Future simulation work addresses the inclusion of nuclear scattering into the micromagnetic approach, which would allow for the computation of polarization-dependent nuclear-magnetic interference terms, yielding a more complete description of the SANS cross section.

Concerning the study of complex systems, it is clear that SANS has played, and continues to play, an important role in the elucidation of nanoscale and microscale magnetic inhomogeneities in complex materials systems. SANS is in fact ideally suited to obtaining bulk-averaged information on magnetic inhomogeneity in such materials and has played a key role in the realization that magnetic and electronic inhomogeneities are widespread across numerous materials classes. Future application of the technique to new materials where such issues are emerging will hopefully take place, including topological magnetic materials, doped magnetic Mott insulators, heavy-fermion systems, or strong spin-orbit oxides such as iridates.

The identification of further compounds with skyrmionic structures and the diverse mechanisms of their stabilization, often connected to topological decay and emergence, is expected to be of high relevance for SANS. Not only the manipulation of SkL phases, but also of noncollinear magnetic structures, by means of electrical currents and fields, microwave radiation, and resulting nonequilibrium physics will be a focus of research, in line with their application in future logical or storage devices. Here multiferroic properties are of particular interest. Apart from SANS, skyrmionic structures in thin films and nanostructured patterns will continue to be of high importance. Despite intense theoretical effort (Barker and Tretiakov, 2016; Zhang, Zhou, and Ezawa, 2016; Göbel et al., 2017), the experimental proof for AF skyrmion states is still lacking. Here key complications are (i) the unambiguous detection of topological properties by means of Hall measurements, which are impossible in the mostly insulating compounds. In particular, recent theoretical work indicates that AF skyrmions show only a topological spin Hall effect (Barker and Tretiakov, 2016). (ii) The high momentum transfer of AF-based SkL structures imposes severe limitations to diffraction measurements, in particular, for SANS. Also the search for skyrmions in magnetically frustrated centrosymmetric materials with high lattice symmetry has recently been the focus of intense theoretical effort (Okubo, Chung, and Kawamura, 2012; Leonov and Motovoy, 2015; Wang et al., 2015; Hayami, Ozawa, and Motome, 2017). Here experimental proof is still scarce.

While SANS has been highly successful for VL studies, important unresolved scientific problems remain, which will require further developments of the technique before they can be addressed. Prominent examples include the prospects for direct imaging of exotic vortex phases, such multiply quantized vortices in bulk superconductors, or the inhomogeneous FuldeFerrell-Larkin-Ovchinnikov state. Such phases often exist in narrow regions of the phase diagram which may be difficult to reach experimentally, suffer from weak scattering from the VL, or be complicated by the need for an unconventional scattering geometry. Attempts to resolve these exotic states may require or be aided by a combination of SANS with techniques that allow an in situ tuning of the superconducting state itself. Importantly, this must extend beyond electric currents and dc or ac magnetic fields, which typically affect only the VL. Of particular interest are the prospects of performing VL studies under uniaxial strain or hydrostatic pressure. Studies of vortex matter have recently been advanced by the use of stroboscopic SANS or TISANE which allow kinematic studies (Mühlbauer et al., 2011) (see next paragraph). These techniques hold great potential as time resolution continues to improve. In addition, spatially resolved SANS studies of, e.g., VL domain formation may be feasible, demonstrated recently by scattering from as few as $10^{6}-10^{7}$ vortices (Louden et al., 2018).

Recent progress in SANS instrumentation regarding TISANE (Gähler and Golub, 1984; Wiedenmann, Keiderling et al., 2006) and the integration of spin-echo techniques (MIEZE) opens up the way to kinetic studies and quasielastic energy resolution. The chopper-based TISANE technique represents an improvement of conventional stroboscopic time-resolved SANS, which is limited by the neutron time-of-flight spread resulting from the wavelength distribution of the incident neutrons to about $300 \mathrm{~Hz}$ time resolution 
(Wiedenmann et al., 2011). TISANE allows one to probe magnetism up to the $\mu$ s regime, which permits the investigation of magnetization dynamics of anisometric nanoparticles in oscillating magnetic fields (Wiedenmann et al., 2008; Bender et al., 2015), the dynamics of vortex (Mühlbauer et al., 2011) and sykrmion lattices (Mühlbauer, Kindervater et al., 2016), or systems out of equilibrium. Regarding theoretical work, the analytical and numerical extension of the present static micromagnetic approach to include magnetization dynamics (Landau-Lifshitz-Gilbert equation) represents a major challenge.

In contrast to kinetic studies of driven dynamics with stroboscopic techniques such as TISANE, the spin-echo variant MIEZE (Gähler, Golub, and Keller, 1992; Hank et al., 1997) allows the combination of the superior $q$ resolution of SANS and the extreme energy resolution of spin echo, in particular, allowing for (ferro)magnetic samples and samples in magnetic fields. MIEZE will open the pathways to study entirely new physics, e.g., FM fluctuations at phase transitions, quantum-phase transitions and frustrated spin liquids, or the melting of SkL and vortex lattices (Kindervater, Säubert, and Böni, 2017; Martin, 2018; Haslbeck et al., 2019).

In view of the upcoming SANS instrumentation at the European Spallation Source (ESS) (Jaksch et al., 2014), it will become possible to study ever smaller samples at extreme environments (temperature, field, pressure). In terms of neutron flux at ESS, the SANS instrument SKADI is estimated to provide a flux of $\approx 5 \times 10^{8} \mathrm{~cm}^{-2} \mathrm{~s}^{-1}$ at $8 \mathrm{~m}$ collimation, which is about a factor of 5 larger than the one of the currently best-performing instrument in the world, the D22 at the ILL (Jaksch et al., 2014; Jaksch, 2019). Moreover, the increased dynamical range will facilitate in situ and in operando measurements of irreversible or kinetic processes.

\section{ACKNOWLEDGMENTS}

We thank Giordano Benacchio, C. Franz, D. Kim, Artem Malyeyev, Mike Manno, Eric McCalla, Jeff Walter, and D. Zákutná for a critical reading of the manuscript. A. M. thanks the National Research Fund of Luxembourg for financial support. E. A. P. acknowledges ABB for its continuous support. S. E. and D. B. acknowledge financial support from the Deutsche Forschungsgemeinschaft (Project No. BE 2464/103), the EU-FP7 project "NANOPYME" (310516), and the EU Horizon-2020 project "AMPHIBIAN" (720853). M. R.E. was supported by the U.S. Department of Energy, Office of Basic Energy Sciences, under Award No. DE-SC0005051. F. B. thanks Andreas Ulbricht for fruitful discussions over the many years. C. L. was funded by the U.S. Department of Energy through the University of Minnesota Center for Quantum Materials under DE-FG02-06ER46275 and DESC-0016371. S. D. acknowledges financial support from the German Research Foundation (DFG Emmy Noether Grant No. DI 1788/2-1).

\section{REFERENCES}

Abrikosov, A. A., 1957, "On the Magnetic Properties of Superconductors of the Second Group," Sov. Phys. JETP 5, 1174-1183.
Adachi, H. M., M. Ishikawa, T. Hirano, M. Ichioka, and K. Machida, 2011, "Origin of Spontaneous Broken Mirror Symmetry of Vortex Lattices in Nb," J. Phys. Soc. Jpn. 80, 113702.

Adachi, K., N. Achiwa, and M. Mekata, 1980, "Helical Magnetic Structure in $\mathrm{CsCuCl}_{3}$," J. Phys. Soc. Jpn. 49, 545-553.

Adams, C. P., J. W. Lynn, Y. M. Mukovskii, A. A. Arsenov, and D. A. Shulyatev, 2000, "Charge ordering and polaron formation in the magnetoresistive oxide $\mathrm{La}_{0.7} \mathrm{Ca}_{0.3} \mathrm{MnO}_{3}$," Phys. Rev. Lett. 85, 3954-3957.

Adams, C. P., J. W. Lynn, V. N. Smolyaninova, A. Biswas, R. L. Greene, W. Ratcliff, S-W. Cheong, Y. M. Mukovskii, and D. A. Shulyatev, 2004, "First-order nature of the ferromagnetic phase transition in (La-Ca) $\mathrm{MnO}_{3}$ near optimal doping," Phys. Rev. B 70, 134414.

Adams, T., 2015, "Neutronenstreuung an Skyrmionengittern in chiralen Magneten," Ph.D. thesis (TU München), unpublished.

Adams, T., A. Chacon, M. Wagner, A. Bauer, G. Brandl, B. Pedersen, H. Berger, P. Lemmens, and C. Pfleiderer, 2012, "LongWavelength Helimagnetic Order and Skyrmion Lattice Phase in $\mathrm{Cu}_{2} \mathrm{OSeO}_{3}$," Phys. Rev. Lett. 108, 237204.

Adams, T., M. Garst, A. Bauer, R. Georgii, and C. Pfleiderer, 2018, "Response of the Skyrmion Lattice in MnSi to Cubic Magnetocrystalline Anisotropies," Phys. Rev. Lett. 121, 187205.

Adams, T., et al., 2011, "Long-Range Crystalline Nature of the Skyrmion Lattice in MnSi," Phys. Rev. Lett. 107, 217206.

Adkins, G. S., C. R. Nappi, and E. Witten, 1983, "Static properties of the Skyrme model," Nucl. Phys. B 228, 552.

Aeppli, G., S. M. Shapiro, R. J. Birgeneau, and H. S. Chen, 1983, "Spin correlations and reentrant spin-glass behavior in amorphous Fe-Mn alloys: Statics," Phys. Rev. B 28, 5160.

Agterberg, D. F., 1998, "Square vortex lattices for two-component superconducting order parameters," Phys. Rev. B 58, 1448414489.

Aharoni, A., 1996, Introduction to the Theory of Ferromagnetism (Clarendon Press, Oxford), 2nd ed.

Ahn, K. H., T. Lookman, and A. R. Bishop, 2004, "Strain-induced metal-insulator coexistence in perovskite manganites," Nature (London) 428, 401.

Alinger, M. J., G. R. Odette, and D. T. Hoelzer, 2004, “The development and stability of Y-Ti-O nanoclusters in mechanically alloyed Fe-Cr based ferritic alloys," J. Nucl. Mater. 329-333, 382-386.

Allen, A.J., 2005, "Characterization of Ceramics by X-Ray and Neutron Small-Angle Scattering," J. Am. Ceram. Soc. 88, 1367-1381.

Allen, A. J., and N. F. Berk, 1994, "Analysis of small-angle scattering data dominated by multiple scattering for systems containing eccentrically shaped particles or pores," J. Appl. Crystallogr. 27, 878-891.

Altstadt, E., E. Keim, H. Hein, M. Serrano, F. Bergner, H.-W. Viehrig, A. Ballesteros, R. Chaouadi, and K. Wilford, 2014, "Fp7 project longlife: Overview of results and implications," Nucl. Eng. Des. 278, 753-757.

Altynbaev, E., S.-A. Siegfried, E. Moskvin, D. Menzel, C. Dewhurst, A. Heinemann, A. Feoktystov, L. Fomicheva, A. Tsvyashchenko, and S. Grigoriev, 2016, "Hidden quantum phase transition in $\mathrm{Mn}_{1-x} \mathrm{Fe}_{x} \mathrm{Ge}$ evidenced by small-angle neutron scattering," Phys. Rev. B 94, 174403.

Amano, Y., M. Ishihara, M. Ichioka, N. Nakai, and K. Machida, 2014, "Eilenberger and London theories for transverse components of flux line lattice form factors in uniaxial superconductors," Phys. Rev. B 90, 144514.

Argyriou, D. N., J. W. Lynn, R. Osborn, B. Campbell, J. F. Mitchell, U. Ruett, H. N. Bordallo, A. Wildes, and C. D. Ling, 2002, "Glass transition in the polaron dynamics of colossal magnetoresistive manganites," Phys. Rev. Lett. 89, 036401. 
Asai, Kichizo, Osamu Yokokura, Nobuhiko Nishimori, Henry Chou, J. M. Tranquada, G. Shirane, Sadao Higuchi, Yuichiro Okajima, and Kay Kohn, 1994, "Neutron-scattering study of the spin-state transition and magnetic correlations in $\mathrm{La}_{1-x} \mathrm{Sr}_{x} \mathrm{CoO}_{3}(x=0$ and 0.08)," Phys. Rev. B 50, 3025-3032.

Aston, D. R., L. W. Dubeck, and F. Rothwarf, 1971, “ 'Intermediate Mixed' State of Type-II Superconductors,” Phys. Rev. B 3, 22312236.

Avdeev, M. V., and V.L. Aksenov, 2010, "Small-angle neutron scattering in structure research of magnetic fluids," Phys. Usp. 53, 971-993.

Avdeev, M. V., V. L. Aksenov, M. Balasoiu, V. M. Garamus, A. Schreyer, Gy Török, L. Rosta, D. Bica, and L. Vékás, 2006, "Comparative analysis of the structure of sterically stabilized ferrofluids on polar carriers by small-angle neutron scattering," J. Colloid Interface Sci. 295, 100-107.

Avdeev, M. V., V. I. Petrenko, A. V. Feoktystov, I. V. Gapon, V. L. Aksenov, L. Vékás, and P. Kopčanský, 2015, "Neutron investigations of ferrofluids," Ukr. J. Phys. 60, 728-736.

Avdeev, Mikhail V., Doina Bica, Ladislau Vékás, Oana Marinica, Maria Balasoiu, Victor L. Aksenov, László Rosta, Vasil M. Garamus, and Andreas Schreyer, 2007, "On the possibility of using short chain length mono-carboxylic acids for stabilization of magnetic fluids," J. Magn. Magn. Mater. 311, 6-9.

Avdeev, Mikhail V., Emmanuelle Dubois, Guillaume Mériguet, Elie Wandersman, Vasil M. Garamus, Artem V. Feoktystov, and Regine Perzynski, 2009, "Small-angle neutron scattering analysis of a water-based magnetic fluid with charge stabilization: Contrast variation and scattering of polarized neutrons," J. Appl. Crystallogr. 42, 1009-1019.

Azhar, Maria, and Maxim Mostovoy, 2017, "Incommensurate spiral order from double-exchange interactions," Phys. Rev. Lett. 118, 027203 .

Bader, S. D., 2006, "Colloquium: Opportunities in nanomagnetism," Rev. Mod. Phys. 78, 1-15.

Bak, P., and M. Høgh Jensen, 1980, "Theory of helical magnetic structures and phase transitions in MnSi and FeGe," J. Phys. C 13, L881.

Bannenberg, L. J., et al., 2017, "Universality of the helimagnetic transition in cubic chiral magnets: Small angle neutron scattering and neutron spin echo spectroscopy studies of FeCoSi," Phys. Rev. B 95, 144433.

Barker, J. G., and J. S. Pedersen, 1995, "Instrumental Smearing Effects in Radially Symmetric Small-Angle Neutron Scattering by Numerical and Analytical Methods," J. Appl. Crystallogr. 28, 105-114.

Barker, Joseph, and Oleg A. Tretiakov, 2016, "Static and Dynamical Properties of Antiferromagnetic Skyrmions in the Presence of Applied Current and Temperature," Phys. Rev. Lett. 116, 147203.

Barrett, Matthew, Andreas Deschner, Jan P. Embs, and Maikel C. Rheinstädter, 2011, "Chain formation in a magnetic fluid under the influence of strong external magnetic fields studied by small angle neutron scattering," Soft Matter 7, 6678.

Bates, F. S., 1988, "Small-angle neutron scattering from amorphous polymers," J. Appl. Crystallogr. 21, 681-691.

Bates, F.S., J.H. Rosedale, and G. H. Fredrickson, 1990, "Fluctuation effects in a symmetric diblock copolymer near the order-disorder transition," J. Chem. Phys. 92, 6255.

Batz, M., S. Baeßler, W. Heil, E. W. Otten, D. Rudersdorf, J. Schmiedeskamp, Y. Sobolev, and M. Wolf, 2005, "3He Spin Filter for Neutrons,” J. Res. Natl. Inst. Stand. Technol. 110, 293.
Bauer, A., M. Garst, and C. Pfleiderer, 2013, "Specific Heat of the Skyrmion Lattice Phase and Field-Induced Tricritical Point in MnSi," Phys. Rev. Lett. 110, 177207.

Bauer, A., A. Neubauer, C. Franz, W. Münzer, M. Garst, and C. Pfleiderer, 2010, "Quantum phase transitions in single-crystal $\mathrm{Mn}_{1-x} \mathrm{Fe}_{x} \mathrm{Si}$ and $\mathrm{Mn}_{1-x} \mathrm{Co}_{x} \mathrm{Si}$ : Crystal growth, magnetization, ac susceptibility, and specific heat," Phys. Rev. B 82, 064404.

Bauer, A., and C. Pfleiderer, 2010, "Generic Aspects of Skyrmion Lattices in Chiral Magnets," in Topological Structures in Ferroic Materials, edited by J. Seidel (Springer, New York), pp. 1-28.

Bauer, A., and C. Pfleiderer, 2012, "Magnetic phase diagram of MnSi inferred from magnetization and ac susceptibility," Phys. Rev. B 85, 214418.

Bauer, Ernst, 2014, Surface Microscopy with Low Energy Electrons (Springer, New York).

Bazhenov, A. N., V. M. Lobashev, A. N. Pirozhkov, and V. N. Slusar, 1993, "An adiabatic resonance spin-flipper for thermal and cold neutrons," Nucl. Instrum. Methods Phys. Res., Sect. A 332, 534.

Bedanta, Subhankar, and Wolfgang Kleemann, 2009, "Supermagnetism," J. Phys. D 42, 013001.

Bellouard, C., I. Mirebeau, and M. Hennion, 1996, "Magnetic correlations of fine ferromagnetic particles studied by small-angle neutron scattering," Phys. Rev. B 53, 5570-5578.

Bender, P., A. Günther, D. Honecker, A. Wiedenmann, S. Disch, A. Tschöpe, A. Michels, and R. Birringer, 2015, "Excitation of $\mathrm{Ni}$ nanorod colloids in oscillating magnetic fields: a new approach for nanosensing investigated by TISANE," Nanoscale 7, 17122-17130.

Bender, P., et al., 2017, "Structural and magnetic properties of multi-core nanoparticles analysed using a generalised numerical inversion method," Sci. Rep. 7, 45990.

Berger, L., Y. Labaye, M. Tamine, and J. M. D. Coey, 2008, "Ferromagnetic nanoparticles with strong surface anisotropy: Spin structures and magnetization processes," Phys. Rev. B 77, 104431.

Bergner, F., M. Lambrecht, A. Ulbricht, and A. Almazouzi, 2010, "Comparative small-angle neutron scattering study of neutronirradiated Fe, Fe-based alloys and a pressure vessel steel,' J. Nucl. Mater. 399, 129-136.

Bergner, F., C. Pareige, V. Kuksenko, L. Malerba, P. Pareige, A. Ulbricht, and A. Wagner, 2013, "Critical assessment of Cr-rich precipitates in neutron-irradiated $\mathrm{Fe}-12$ at $\% \mathrm{Cr}$ : Comparison of SANS and APT," J. Nucl. Mater. 442, 463-469.

Bergner, F., A. Ulbricht, H. Hein, and M. Kammel, 2008, "Flux dependence of cluster formation in neutron-irradiated weld material," J. Phys. Condens. Matter 20, 104262.

Bergner, F., A. Ulbricht, and C. Heintze, 2009, "Estimation of the solubility limit of $\mathrm{Cr}$ in $\mathrm{Fe}$ at $300{ }^{\circ} \mathrm{C}$ from small-angle neutron scattering in neutron-irradiated Fe-Cr alloys," Scr. Mater. 61, 1060-1063.

Berkov, D. V., 2007, in Handbook of Magnetism and Advanced Magnetic Materials, edited by H. Kronmüller and S. Parkin (Wiley, New York), Vol. 2, pp. 795-823.

Berkov, D. V., and N. L. Gorn, 1998, "Quasistatic remagnetization processes in two-dimensional systems with random on-site anisotropy and dipolar interaction: Numerical simulations," Phys. Rev. B 57, 14332.

Berkov, D. V, and N.L. Gorn, 2005, in Handbook of Advanced Magnetic Materials, edited by Y. Liu, D. Shindo, and D. J. Sellmyer (Springer Verlag and Tsinghua University Press, New York), pp. 421-507.

Bhatti, Kanwal Preet, S. El-Khatib, Vijay Srivastava, R. D. James, and C. Leighton, 2012, "Small-angle neutron scattering study of magnetic ordering and inhomogeneity across the martensitic phase 
transformation in $\mathrm{Ni}_{50-x} \mathrm{Co}_{x} \mathrm{Mn}_{40} \mathrm{Sn}_{10}$ alloys," Phys. Rev. B 85, 134450.

Bhatti, K. P., V. Srivastava, D. P. Phelan, R. D. James, and C. Leighton, 2016, Chap. Magnetic phase competition in off-stoichiometric martensitic Heusler alloys: The $\mathrm{Ni}_{50-x} \mathrm{Co}_{x} \mathrm{Mn}_{40} \mathrm{Sn}_{10}$ system, in Heusler Alloys: Properties, Growth, Applications (Springer, New York), pp. 193-216.

Bianchi, A. D., et al., 2008, "Superconducting vortices in $\mathrm{CeCoIn}_{5}$ : Towards the Pauli-limiting field," Science 319, 177-180.

Bick, J.-P., K. Suzuki, E. P. Gilbert, E. M. Forgan, R. Schweins, P. Lindner, C. Kübel, and A. Michels, 2013, "Exchange-stiffness constant of a $\mathrm{Nd}-\mathrm{Fe}-\mathrm{B}$ based nanocomposite determined by magnetic neutron scattering," Appl. Phys. Lett. 103, 122402.

Bick, J.-P., et al., 2013, "Magnetization reversal in Nd-Fe-B based nanocomposites as seen by magnetic small-angle neutron scattering," Appl. Phys. Lett. 102, 022415.

Biotteau, G., M. Hennion, F. Moussa, J. Rodríguez-Carvajal, L. Pinsard, A. Revcolevschi, Y.M. Mukovskii, and D. Shulyatev, 2001, "Approach to the metal-insulator transition in $\mathrm{La}_{1 x} \mathrm{Ca}_{x} \mathrm{MnO}_{3}(0 \lesssim x \lesssim 0.2)$ : Magnetic inhomogeneity and spinwave anomaly," Phys. Rev. B 64, 104421.

Bischof, M., P. Staron, A. Michels, P. Granitzer, K. Rumpf, H. Leitner, C. Scheu, and H. Clemens, 2007, "The influence of spinmisalignment scattering on the SANS data evaluation of martensitic age-hardening steels," Acta Mater. 55, 2637-2646.

Blatter, G., M. V. Feigel'man, V. B. Geshkenbein, A. I. Larkin, and V. M. Vinokur, 1994, "Vortices in high-temperature superconductors," Rev. Mod. Phys. 66, 1125-1388.

Bley, F., 1992, "Neutron small-angle scattering study of unmixing in Fe-Cr alloys," Acta Metall. Mater. 40, 1505-1517.

Blume, M., 1963, "Polarization Effects in the Magnetic Elastic Scattering of Slow Neutrons," Phys. Rev. 130, 1670.

Bogdanov, A., and A. Hubert, 1994, "Thermodynamically stable magnetic vortex states in magnetic crystals," J. Magn. Magn. Mater. 138, 255.

Bogdanov, A. N., 1995, "New localized solutions of the nonlinear field equations," Sov. Phys. JETP 62, 247.

Bogdanov, A. N., and U. K. Rößler, 2001, "Chiral Symmetry Breaking in Magnetic Thin Films and Multilayers," Phys. Rev. Lett. 87, 037203.

Bogdanov, A. N., U. K. Rößler, M. Wolf, and K.-H. Müller, 2002, "Magnetic structures and reorientation transitions in noncentrosymmetric uniaxial antiferromagnets," Phys. Rev. B 66, 214410.

Bogdanov, A. N., and D. A. Yablonskii, 1989, “Thermodynamically stable "vortices" in magnetically ordered crystals. The mixed state of magnets," Sov. Phys. JETP 68, 101 [ZhETF 95, 178 (1989)].

Böhmert, J., H.-W. Viehrig, and A. Ulbricht, 2004, "Correlation between irradiation-induced changes of microstructural parameters and mechanical properties of RPV steels," J. Nucl. Mater. 334, 71-78.

Bonini, Massimo, Sebastian Lenz, Ester Falletta, Francesca Ridi, Emiliano Carretti, Emiliano Fratini, Albrecht Wiedenmann, and Piero Baglioni, 2008, "Acrylamide-based magnetic nanosponges: A new smart nanocomposite material," Langmuir 24, 12644-12650.

Bonini, Massimo, Albrecht Wiedenmann, and Piero Baglioni, 2004, "Small angle polarized neutrons (SANSPOL) investigation of surfactant free magnetic fluid of uncoated and silica-coated cobalt-ferrite nanoparticles," J. Phys. Chem. B 108, 14901-14906.

Bonini, Massimo, Albrecht Wiedenmann, and Piero Baglioni, 2006, "Synthesis and characterization of magnetic nanoparticles coated with a uniform silica shell," Mater. Sci. Eng. C 26, 745-750.
Bonini, Massimo, Albrecht Wiedenmann, and Piero Baglioni, 2007, "Study of ferrite ferrofluids by small-angle scattering of polarized neutrons," J. Appl. Crystallogr. 40, s254.

Bonny, G., D. Terentyev, and L. Malerba, 2008, "On the $\alpha-\alpha^{\prime}$ miscibility gap of Fe-Cr alloys," Scr. Mater. 59, 1193-1196.

Bordacs, S., et al., 2017, "Equilibrium Skyrmion lattice Ground State in a Polar Easy-Plane Magnet," Sci. Rep. 7, 7584.

Bos, Jan-Willem G., Claire V. Colin, and Thomas T. M. Palstra, 2008, "Magnetoelectric coupling in the cubic ferrimagnet $\mathrm{Cu}_{2} \mathrm{OSeO}_{3}$," Phys. Rev. B 78, 094416.

Boulle, Olivier, et al., 2016, "Room-temperature chiral magnetic skyrmions in ultrathin magnetic nanostructures," Nat. Nanotechnol. 11, 449-454.

Bowell, C. J., 2008, "Studies of the vortex lattice using complementary small angle neutron scattering and heat capacity measurements," Ph.D. thesis (University of Birmingham).

Bowell, C. J., R. J. Lycett, M. Laver, C. D. Dewhurst, R. Cubitt, and E. M. Forgan, 2010, "Absence of vortex lattice melting in a highpurity Nb superconductor,” Phys. Rev. B 82, 144508.

Brandt, E. H., 1995, "The Flux-Line-Lattice in Superconductors," Rep. Prog. Phys. 58, 1465-1594.

Bray, A. J., 1987, "Nature of the griffiths phase," Phys. Rev. Lett. 59, 586-589.

Brazovskii, S. A., 1975, "Phase transition of an isotropic system to a nonuniform state," Sov. Phys. JETP 41, 85 [ZhETF 68, 175 (1975)].

Brazovskii, S. A., I. E. Dzyaloshinskii, and A. R. Muratov, 1987, "The theory of weak crystallization," Sov. Phys. JETP 66, 625.

Brazovskii, S. A., and S. G. Dmitriev, 1975, "Phase transitions in cholesteric liquid crystals," Sov. Phys. JETP 42, 497.

Breßler, I., J. Kohlbrecher, and A. F. Thünemann, 2015, "SASfit: a tool for small-angle scattering data analysis using a library of analytical expressions," J. Appl. Crystallogr. 48, 1587-1598.

Briggs, S. A., P. D. Edmondson, K. C. Littrell, Y. Yamamoto, R. H. Howard, C. R. Daily, K. A. Terrani, K. Sridharan, and K. G. Field, 2017, "A combined APT and SANS investigation of $\alpha^{\prime}$ phase precipitation in neutron-irradiated model FeCrAl alloys," Acta Mater. 129, 217-228.

Brown, G. E., and M. Rho, 2010, Eds., The Multifaceted Skyrmion (World Scientific, Singapore).

Brown, P. J., 2006, in Neutron Scattering from Magnetic Materials, edited by T. Chatterji (Elsevier, New York), pp. 215-244.

Brown, S. P., D. Charalambous, E. C. Jones, E. M. Forgan, P. G. Kealey, A. Erb, and J. Kohlbrecher, 2004, "Triangular to square flux lattice phase transition in $\mathrm{YBa}_{2} \mathrm{Cu}_{3} \mathrm{O}_{7}$," Phys. Rev. Lett. 92, 067004.

Brown, Jr., W. F., 1963, Micromagnetics (Interscience Publishers, New York).

Butler, P. A., M. Doucet, A. Jackson, and S. King, 2016, SasView for Small Angle Scattering Analysis, www.sasview.org.

Butter, Karen, Armin Hoell, Albrecht Wiedenmann, Andrei V. Petukhov, and Gert Jan Vroege, 2004, "Small-angle neutron and X-ray scattering of dispersions of oleic-acid-coated magnetic iron particles," J. Appl. Crystallogr. 37, 847-856.

Bykov, A. A., Yu. O. Chetverikov, A. N. Pirogov, and S. V. Grigoriev, 2015, "Quasi-Two-Dimensional Character of the Magnetic OrderDisorder Transition in $\mathrm{YMn}_{6} \mathrm{Sn}_{6}$," JETP Lett. 101, 699-702.

Caciuffo, R., J. Mira, J. Rivas, M. A. Señarís-Rodríguez, P. G. Radaelli, F. Carsughi, D. Fiorani, and J. B. Goodenough, 1999, "Transition from itinerant to polaronic conduction in $\mathrm{La}_{1-x} \mathrm{Sr}_{x} \mathrm{CoO}_{3}$ perovskites," Europhys. Lett. 45, 399.

Caciuffo, R., D. Rinaldi, G. Barucca, J. Mira, J. Rivas, M. A. Señarís Rodríguez, P. G. Radaelli, D. Fiorani, and J. B. Goodenough, 1999, 
"Structural details and magnetic order of $\mathrm{La}_{1-x} \mathrm{Sr}_{x} \mathrm{CoO}_{3}(x \lesssim 0.3)$," Phys. Rev. B 59, 1068-1078.

Cameron, A. S., Y. V Tymoshenko, P. Y. Portnichenko, J. Gavilano, V. Tsurkan, V. Felea, A. Loidl, S. Zherlitsyn, J. Wosnitza, and D. S. Inosov, 2016, "Magnetic phase diagram of the helimagnetic spinel compound $\mathrm{ZnCr}_{2} \mathrm{Se}_{4}$ revisited by small-angle neutron scattering," J. Phys. Condens. Matter 28, 146001.

Campbell, L. J., M. M. Doria, and V. G. Kogan, 1988, "Vortex lattice structures in uniaxial superconductors," Phys. Rev. B 38, 2439.

Carter, R. G., N. Soneda, K. Dohi, J. M. Hyde, C. A. English, and W. L. Server, 2001, "Microstructural characterization of irradiationinduced $\mathrm{Cu}$-enriched clusters in reactor pressure vessel steels," J. Nucl. Mater. 298, 211-224.

Chacon, A., A. Bauer, T. Adams, F. Rucker, G. Brandl, R. Georgii, M. Garst, and C. Pfleiderer, 2015, "Uniaxial pressure dependence of magnetic order in MnSi," Phys. Rev. Lett. 115, 267202.

Chacon, A., L. Heinen, M. Halder, A. Bauer, W. Simeth, S. Mühhlbauer, H. Berger, M. Garst, A. Rosch, and C. Pfleiderer, 2018, "Observation of two independent skyrmion phases in a chiral magnetic material," Nat. Phys. 14, 936-941.

Champel, T., and V.P Mineev, 2001, "Theory of Equilibrium Flux Lattice in $\mathrm{UPt}_{3}$ under Magnetic Field Parallel to Hexagonal Crystal Axis," Phys. Rev. Lett. 86, 4903-4906.

Chatterji, T., 2006, Ed., Neutron Scattering from Magnetic Materials (Elsevier, New York).

Chen, S.-H., and T.-L. Lin, 1987, in Methods of Experimental Physics-Neutron Scattering, edited by D. L. Price and K. Sköld (Academic Press, New York), Vol. 23, Pt. B, pp. 489-543.

Chen, S. H., E. Y. Sheu, J. Kalus, and H. Hoffmann, 1988, "Smallangle neutron scattering investigation of correlations in charged macromolecular and supramolecular solutions," J. Appl. Crystallogr. 21, 751-769.

Cheng, X. M., and D. J. Keavney, 2012, "Studies of nanomagnetism using synchrotron-based X-ray photoemission electron microscopy (X-PEEM)," Rep. Prog. Phys. 75, 026501.

Cheong, S.-W., P. A. Sharma, N. Hur, Y. Horibe, and C. H. Chen, 2002, "Electronic phase separation in complex materials," Physica B (Amsterdam) 318, 39-51.

Chovan, J., M. Marder, and N. Papanicolaou, 2013, "Field-induced phase transitions in the helimagnet $\mathrm{Ba}_{2} \mathrm{CuGe}_{2} \mathrm{O}_{7}$," Phys. Rev. B 88, 064421.

Chovan, J., N. Papanicolaou, and S. Komineas, 2002, "Intermediate phase in the spiral antiferromagnet $\mathrm{Ba}_{2} \mathrm{CuGe}_{2} \mathrm{O}_{7}$," Phys. Rev. B 65, 064433.

Christen, D. K., H. R. Kerchner, S. T. Sekula, and Y. K. Chang, 1985, "Flux-line lattice anisotropy in $\mathrm{V}_{3} \mathrm{Si}$ : observation and interpretation," Physica B+C (Amsterdam) 135, 369-373.

Christen, D. K., H. R. Kerchner, S. T. Sekula, and P. Thorel, 1980, "Equilibrium properties of the fluxoid lattice in single-crystal niobium. II. Small-angle neutron-diffraction measurements," Phys. Rev. B 21, 102-117.

Christen, D. K., F. Tasset, S. Spooner, and H. A. Mook, 1977, "Study of Intermediate Mixed State of Niobium by Small-Angle NeutronScattering," Phys. Rev. B 15, 4506-4509.

Chung, J.-H., M. Matsuda, S.-H. Lee, K. Kakurai, H. Ueda, T. J. Sato, H. Takagi, K.-P. Hong, and S. Park, 2005, "Statics and dynamics of incommensurate spin order in a geometrically frustrated antiferromagnet $\mathrm{CdCr}_{2} \mathrm{O}_{4}$," Phys. Rev. Lett. 95, 247204.

Ciccariello, S., J. Goodisman, and H. Brumberger, 1988, "On the Porod Law," J. Appl. Crystallogr. 21, 117-128.

Clem, J. R., 1975, "Simple Model for Vortex Core in a Type-II Superconductor,” J. Low Temp. Phys. 18, 427-435.
Coey, J. M. D., 1971, "Noncolinear Spin Arrangement in Ultrafine Ferrimagnetic Crystallites," Phys. Rev. Lett. 27, 1140-1142.

Coey, J. M. D., 1987, "Noncollinear spin structures," Can. J. Phys. 65, 1210-1232.

Coey, J. M. D., M. Viret, and S. von Molnár, 1999, "Mixed-valence manganites," Adv. Phys. 48, 167-293.

Cong, D. Y., S. Roth, J. Liu, Q. Luo, M. Pötschke, C. Hürrich, and L. Schultz, 2010, "Superparamagnetic and superspin glass behaviors in the martensitic state of $\mathrm{Ni}_{43.5} \mathrm{Co}_{6.5} \mathrm{Mn}_{39} \mathrm{Sn}_{11}$ magnetic shape memory alloy," Appl. Phys. Lett. 96, 112504.

Cong, D. Y., S. Roth, and L. Schultz, 2012, "Magnetic properties and structural transformations in $\mathrm{Ni}-\mathrm{Co}-\mathrm{Mn}-\mathrm{Sn}$ multifunctional alloys," Acta Mater. 60, 5335-5351.

Coppola, R., R. Kampmann, M. Magnani, and P. Staron, 1998, "Microstructural investigation, using polarized neutron scattering, of a martensitic steel for fusion reactors," Acta Mater. 46, 54475456.

Coqblin, B., 1977, The Electronic Structure of Rare-Earth Metals and Alloys: The magnetic Heavy Rare-Earths (Academic Press, New York).

Cribier, D., B. Jacrot, L. M. Rao, and B. Farnoux, 1964, "Mise en evidence par diffraction de neutrons d'une structure periodique du champ magnetique dans le niobium supraconducteur," Phys. Lett. 9, 106-107.

Cubitt, R., M. R. Eskildsen, C. D. Dewhurst, J. Jun, S. M. Kazakov, and J. Karpinski, 2003, "Effects of two-band superconductivity on the flux-line lattice in magnesium diboride," Phys. Rev. Lett. 91, 047002 .

Cubitt, R., et al., 1993, "Direct Observation of Magnetic-Flux Lattice Melting and Decomposition in the High- $T_{c}$ Superconductor $\mathrm{Bi}_{2.15} \mathrm{Sr}_{1.95} \mathrm{CaCu}_{2} \mathrm{O}_{8+x}$," Nature (London) 365, 407-411.

Cui, J., et al., 2006, "Combinatorial search of thermoelastic shapememory alloys with extremely small hysteresis width," Nat. Mater. 5, 286-290.

Curiale, J., M. Granada, H. E. Troiani, R. D. Sánchez, A. G. Leyva, P. Levy, and K. Samwer, 2009, "Magnetic dead layer in ferromagnetic manganite nanoparticles,” Appl. Phys. Lett. 95, 043106.

Dagotto, Elbio, 2002, Nanoscale Phase Separation and Colossal Magnetoresistance (Springer, New York).

Dagotto, Elbio, Takashi Hotta, and Adriana Moreo, 2001, "Colossal magnetoresistant materials: the key role of phase separation," Phys. Rep. 344, 1-153.

Dai, Pengcheng, J. A. Fernandez-Baca, N. Wakabayashi, E. W. Plummer, Y. Tomioka, and Y. Tokura, 2000, "Short-range polaron correlations in the ferromagnetic $\mathrm{La}_{1-x} \mathrm{Ca}_{x} \mathrm{MnO}_{3}$," Phys. Rev. Lett. 85, 2553-2556.

Daniilidis, N. D., S. R. Park, I. K. Dimitrov, J. W. Lynn, and X. S. Ling, 2007, "Emergence of Quasi-Long-Range Order below the Bragg Glass Transition," Phys. Rev. Lett. 99, 147007.

Das, P., J. M. Densmore, C. Rastovski, K. J. Schlesinger, M. Laver, C. D. Dewhurst, K. Littrell, S. L. Bud'ko, P. C. Canfield, and M. R. Eskildsen, 2012, "Field dependence of the superconducting basal plane anisotropy of $\mathrm{TmNi}_{2} \mathrm{~B}_{2} \mathrm{C}$," Phys. Rev. B 86, 144501.

Das, P., C. Rastovski, T.R. O’Brien, K. J. Schlesinger, C. D. Dewhurst, L. DeBeer-Schmitt, N. D. Zhigadlo, J. Karpinski, and M. R. Eskildsen, 2012, “Observation of Well-Ordered Metastable Vortex Lattice Phases in Superconducting $\mathrm{MgB}_{2}$ Using SmallAngle Neutron Scattering," Phys. Rev. Lett. 108, 167001.

Das, P., et al., 2012, "Vortex Lattice Studies in CeCoIn ${ }_{5}$ with $H \perp c$," Phys. Rev. Lett. 108, 087002.

DeBeer-Schmitt, L., C. D. Dewhurst, B. W. Hoogenboom, C. Petrovic, and M. R. Eskildsen, 2006, "Field dependent coherence 
length in the superclean, high- $\kappa$ superconductor CeCoIn 5 ," Phys. Rev. Lett. 97, 127001.

DeBeer-Schmitt, L., M. R. Eskildsen, M. Ichioka, K. Machida, N. Jenkins, C. D. Dewhurst, A. B. Abrahamsen, S. L. Bud'ko, and P. C. Canfield, 2007, "Pauli paramagnetic effects on vortices in superconducting $\mathrm{TmNi}_{2} \mathrm{~B}_{2}$ C," Phys. Rev. Lett. 99, 167001.

Debye, P., H. R. Anderson, and H. Brumberger, 1957, "Scattering by an Inhomogeneous Solid. II. The Correlation Function and Its Application,” J. Appl. Phys. 28, 679-683.

Debye, P., and A. M. Bueche, 1949, "Scattering by an Inhomogeneous Solid," J. Appl. Phys. 20, 518-525.

de Gennes, P.-G., and J. Matricon, 1964, "Collective Modes of Vortex Lines in Superconductors of the Second Kind," Rev. Mod. Phys. 36, 45-49.

Deisenhofer, J., et al., 2005, "Observation of a griffiths phase in paramagnetic $\mathrm{La}_{1-x} \mathrm{Sr}_{x} \mathrm{MnO}_{3}$," Phys. Rev. Lett. 95, 257202.

Delagnes, D., F. Pettinari-Sturmel, M. H. Mathon, R. Danoix, F. Danoix, C. Bellot, P. Lamesle, and A. Grellier, 2012, "Cementitefree martensitic steels: A new route to develop high strength/high toughness grades by modifying the conventional precipitation sequence during tempering," Acta Mater. 60, 5877-5888.

Dennis, Cindi L., Kathryn L. Krycka, Julie A. Borchers, Ryan D. Desautels, Johan Van Lierop, Natalie F. Huls, Andrew J. Jackson, Cordula Gruettner, and Robert Ivkov, 2015, "Internal Magnetic Structure of Nanoparticles Dominates Time-Dependent Relaxation Processes in a Magnetic Field," Adv. Funct. Mater. 25, 4300-4311.

Densmore, J. M., et al., 2009, "Small-angle neutron scattering study of the vortex lattice in superconducting $\mathrm{LuNi}_{2} \mathrm{~B}_{2} \mathrm{C}$," Phys. Rev. B 79, 174522.

De Teresa, J. M., P. A. Algarabel, C. Ritter, J. Blasco, M. R. Ibarra, L. Morellon, J. I. Espeso, and J.C. Gómez-Sal, 2005, "Possible quantum critical point in $\mathrm{La}_{2 / 3} \mathrm{Ca}_{1 / 3} \mathrm{Mn}_{1-x} \mathrm{Ga}_{x} \mathrm{O}_{3}$," Phys. Rev. Lett. 94, 207205.

De Teresa, J. M., M. R. Ibarra, P. A. Algarabel, C. Ritter, C. Marquina, J. Blasco, J. Garcia, A. del Moral, and Z. Arnold, 1997, "Evidence for magnetic polarons in the magnetoresistive perovskites," Nature (London) 386, 256-259.

De Teresa, J. M., et al., 2002, "Magnetic versus orbital polarons in colossal magnetoresistance manganites," Phys. Rev. B 65, 100403.

Dewhurst, C. D., 2008, "D33-a third small-angle neutron scattering instrument at the Institut Laue Langevin,” Meas. Sci. Technol. 19, 034007.

Dewhurst, C. D., 2016, Graphical Reduction and Analysis SANS Program for MatlabTM, https://www.ill.eu/instruments-support/ instruments-groups/groups/lss/grasp/home/.

Dewhurst, C. D., I. Grillo, D. Honecker, M. Bonnaud, M. Jacques, C. Amrouni, A. Perillo-Marcone, G. Manzin, and R. Cubitt, 2016, "The small-angle neutron scattering instrument D33 at the Institut Laue-Langevin,” J. Appl. Crystallogr. 49, 1-14.

Dewhurst, C. D., S. J. Levett, and D. McK. Paul, 2005, "Vortexlattice symmetry near $T_{c}$ in $\mathrm{YNi}_{2} \mathrm{~B}_{2} \mathrm{C}$," Phys. Rev. B 72, 014542.

Disch, S., E. Wetterskog, R. P. Hermann, A. Wiedenmann, U. Vainio, G. Salazar-Alvarez, L. Bergström, and Th. Brückel, 2012, "Quantitative spatial magnetization distribution in iron oxide nanocubes and nanospheres by polarized small-angle neutron scattering," New J. Phys. 14, 013025.

Döbrich, F., J. Kohlbrecher, M. Sharp, H. Eckerlebe, R. Birringer, and A. Michels, 2012, "Neutron scattering study of the magnetic microstructure of nanocrystalline gadolinium," Phys. Rev. B 85, 094411.

Doiron-Leyraud, N., I. R. Walker, L. Taillefer, M. J. Steiner, S. R. Julian, and G. G. Lonzarich, 2003, "Fermi-liquid breakdown in the paramagnetic phase of a pure metal," Nature (London) 425, 595-599.

Drittler, B., N. Stefanou, S. Blügel, R. Zeller, and P. H. Dederichs, 1989, "Electronic structure and magnetic properties of dilute Fe alloys with transition-metal impurities," Phys. Rev. B 40, 8203-8212.

Dutta, P., S. Pal, M. S. Seehra, N. Shah, and G. P. Huffman, 2009, "Size dependence of magnetic parameters and surface disorder in magnetite nanoparticles," J. Appl. Phys. 105, 07B501.

Dzyaloshinskii, I.E., 1958, "A thermodynamic theory of weak ferromagnetism of antiferromagnets," J. Phys. Chem. Solids 4, 241.

Eidenberger, E., R. Schnitzer, G. A. Zickler, M. Schober, M. Bischof, P. Staron, H. Leitner, A. Schreyer, and H. Clemens, 2011, "Applications of photons and neutrons for the characterization and development of advanced steels," Adv. Eng. Mater. 13, 664-673.

El-Khatib, S., Shameek Bose, C. He, J. Kuplic, M. Laver, J. A. Borchers, Q. Huang, J. W. Lynn, J. F. Mitchell, and C. Leighton, 2010, "Spontaneous formation of an exchange-spring composite via magnetic phase separation in $\operatorname{Pr}_{1-x} \mathrm{Ca}_{x} \mathrm{CoO}_{3}$," Phys. Rev. B 82, 100411.

El-Khatib, S., D. Phelan, J. G. Barker, H. Zheng, J. F. Mitchell, and C. Leighton, 2015, "Neutron-scattering-based evidence for interacting magnetic excitons in $\mathrm{LaCoO}_{3}$," Phys. Rev. B 92, 060404.

Enz, U., 1961, "Magnetization process of a helical spin configuration," J. Appl. Phys. 32, S22.

Erokhin, S., D. Berkov, N. Gorn, and A. Michels, 2011, "New Methodology for Micromagnetic Simulations of Nanocomposites With Applications to SANS Experiments," IEEE Trans. Magn. 47, 3044-3047.

Erokhin, S., D. Berkov, M. Ito, A. Kato, M. Yano, and A. Michels, 2018, "Towards understanding of magnetization reversal in Nd-Fe-B nanocomposites: analysis by high-throughput micromagnetic simulations," J. Phys. Condens. Matter 30, 125802.

Erokhin, Sergey, and Dmitry Berkov, 2017, "Optimization of nanocomposite materials for permanent magnets: Micromagnetic simulations of the effects of intergrain exchange and the shapes of hard grains," Phys. Rev. Applied 7, 014011.

Erokhin, Sergey, Dmitry Berkov, Nataliya Gorn, and Andreas Michels, 2012a, "Magnetic neutron scattering on nanocomposites: Decrypting cross-section images using micromagnetic simulations," Phys. Rev. B 85, 134418.

Erokhin, Sergey, Dmitry Berkov, Nataliya Gorn, and Andreas Michels, 2012b, "Micromagnetic modeling and small-angle neutron scattering characterization of magnetic nanocomposites," Phys. Rev. B 85, 024410.

Erokhin, Sergey, Dmitry Berkov, and Andreas Michels, 2015, "Dipolar spin-misalignment correlations in inhomogeneous magnets: Comparison between neutron scattering and micromagnetic approaches," Phys. Rev. B 92, 014427.

Eskildsen, M. R., 2011, "Vortex lattices in type-II superconductors studied by small-angle neutron scattering," Front. Phys. 6, 398-409.

Eskildsen, M. R., E. M. Forgan, and H. Kawano-Furukawa, 2011, "Vortex structures, penetration depth and pairing in iron-based superconductors studied by small-angle neutron scattering," Rep. Prog. Phys. 74, 124504.

Eskildsen, M. R., et al., 1997, "Observation of a field-driven structural phase transition in the flux line lattice in $\mathrm{ErNi}_{2} \mathrm{~B}_{2} \mathrm{C}$," Phys. Rev. Lett. 78, 1968-1971.

Eskildsen, M. R., et al., 1998, "Intertwined symmetry of the magnetic modulation and the flux-line lattice in the superconducting state of $\mathrm{TmNi}_{2} \mathrm{~B}_{2} \mathrm{C}$," Nature (London) 393, 242-245. 
Eskildsen, M. R., et al., 2009, "Vortices in superconducting $\mathrm{Ba}\left(\mathrm{Fe}_{0.93} \mathrm{Co}_{0.07}\right)_{2} \mathrm{As}_{2}$ studied via small-angle neutron scattering and Bitter decoration," Phys. Rev. B 79, 100501.

Everschor, Karin, Markus Garst, Benedikt Binz, Florian Jonietz, Sebastian Mühlbauer, Christian Pfleiderer, and Achim Rosch, 2012, "Rotating skyrmion lattices by spin torques and field or temperature gradients," Phys. Rev. B 86, 054432.

Farrell, D. F., Y. Ijiri, C. V. Kelly, J. A. Borchers, J. J. Rhyne, Y. Ding, and S. A. Majetich, 2006, "Small angle neutron scattering study of disordered and crystalline iron nanoparticle assemblies," J. Magn. Magn. Mater. 303, 318-322.

Fath, M., S. Freisem, A. A. Menovsky, Y. Tomioka, J. Aarts, and J. A. Mydosh, 1999, "Spatially inhomogeneous metal-insulator transition in doped manganites," Science 285, 1540.

Feigin, L. A., and D. I. Svergun, 1987, Structure Analysis by SmallAngle X-Ray and Neutron Scattering (Plenum Press, New York).

Felber, J., R. Gaehler, R. Golub, and K. Prechtel, 1998, "Coherence volumes and neutron scattering," Physica B (Amsterdam) 252, 34-43.

Felea, V., S. Yasin, A. Günther, J. Deisenhofer, H.-A. Krug von Nidda, S. Zherlitsyn, V. Tsurkan, P. Lemmens, J. Wosnitza, and A. Loidl, 2012, "Spin-lattice coupling in the frustrated antiferromagnet $\mathrm{ZnCr}_{2} \mathrm{Se}_{4}$ probed by ultrasound," Phys. Rev. B 86, 104420.

Felser, C., and A. Hirohata, 2015, Heusler alloys: Properties, growth and applications (Springer, New York).

Fiebig, M., D. Fröhlich, K. Kohn, St. Leute, Th. Lottermoser, V. V. Pavlov, and R. V. Pisarev, 2000, "Determination of the magnetic symmetry of hexagonal manganites by second harmonic generation," Phys. Rev. Lett. 84, 5620-5623.

Fine, M. E., J. Z. Liu, and M. D. Asta, 2007, “An unsolved mystery: The composition of bcc $\mathrm{Cu}$ alloy precipitates in bcc Fe and steels," Mater. Sci. Eng. A 463, 271-274.

Fitzsimmons, M. R., et al., 2004, "Neutron scattering studies of nanomagnetism and artificially structured materials," J. Magn. Magn. Mater. 271, 103-146.

Fobes, D. M., Luo Yongkang, N. Leon-Brito, E. D. Bauer, V. R. Fanelli, M. A. Taylor, L. M. DeBeer-Schmitt, and M. Janoschek, 2017, "Versatile strain-tuning of modulated long-period magnetic structures," Appl. Phys. Lett. 110, 192409.

Fobes, D. M., et al., 2018, "Tunable emergent heterostructures in a prototypical correlated metal," Nat. Phys. 14, 456-460.

Forgan, E. M., E. P. Gibbons, K. A. McEwen, and D. Fort, 1989, "Observation of a quadruple- $q$ magnetic structure in neodymium," Phys. Rev. Lett. 62, 470-473.

Forgan, E. M., S. J. Levett, P. G. Kealey, R. Cubitt, C. D. Dewhurst, and D. Fort, 2002, "Intrinsic behavior of flux lines in pure niobium near the upper critical field," Phys. Rev. Lett. 88, 167003.

Forgan, E. M., D. McK. Fault, H. A. Mook, P. A. Timmins, H. Keller, S. Sutton, and J. S. Abell, 1990, "Observation by neutron diffraction of the magnetic flux lattice in single-crystal $\mathrm{YBa}_{2} \mathrm{Cu}_{3} \mathrm{O}_{7-\delta}$," Nature (London) 343, 735-737.

Franz, M., I. Affleck, and M. Amin, 1997, “Theory of Equilibrium Flux Lattices in Unconventional Superconductors," Phys. Rev. Lett. 79, 1555-1558.

Fratzl, P., 2003, "Small-angle scattering in materials science-a short review of applications in alloys, ceramics and composite materials," J. Appl. Crystallogr. 36, 397-404.

Freeman, A. J., and Kohji Nakamura, 2004, "Modern computational magnetism: role of noncollinear magnetism in complex magnetic phenomena," Phys. Status Solidi B 241, 1399-1405.

Frisius, F., and D. Buenemann, 1979, "The measurement of radiation defects in iron alloys by means of the small angle neutron scattering," in Proceedings of the International Conference on Irradiation
Behaviour of Metallic Materials for Fast Reactor Core Components, edited by J. Poirier and J. M. Dupouy (CEA Saclay), pp. 247-252.

Fritz, G., and O. Glatter, 2006, "Structure and interaction in dense colloidal systems: evaluation of scattering data by the generalized indirect Fourier transformation method," J. Phys. Condens. Matter 18, S2403-S2419.

Fritz-Popovski, G., 2015, "Interpretation of two-dimensional realspace functions obtained from small-angle scattering data of oriented microstructures," J. Appl. Crystallogr. 48, 44-51.

$\mathrm{Fu}$, Zhendong, Yinguo Xiao, Artem Feoktystov, Vitaliy Pipich, Marie-Sousai Appavou, Yixi Su, Erxi Feng, Wentao Jin, and Thomas Brückel, 2016, "Field-induced self-assembly of iron oxide nanoparticles investigated using small-angle neutron scattering," Nanoscale 8, 18541-18550.

Fujii, H., M. Saga, T. Takeda, S. Komura, T. Okamoto, S. Hirosawa, and M. Sagawa, 1987, "Small angle neutron scattering from $\mathrm{Nd}_{15} \mathrm{Fe}_{77} \mathrm{~B}_{8}^{11}$ and $\mathrm{Nd}_{15} \mathrm{Fe}_{76} \mathrm{Al}_{1} \mathrm{~B}_{8}^{11}$ permanent magnets," IEEE Trans. Magn. 23, 3119-3121.

Furrer, A., J. Mesot, and T. Strässle, 2009, Neutron Scattering in Condensed Matter Physics (World Scientific, Singapore).

Furrer, Albert, and Oliver Waldmann, 2013, "Magnetic cluster excitations," Rev. Mod. Phys. 85, 367-420.

Gähler, R., and R. Golub, 1984, "Time dependent neutron opticsQuantum mechanical effects on beam chopping and a new type of high resolution neutron spectrometer (FOTOF)," Z. Phys. B 56, $5-12$.

Gähler, R., R. Golub, and T. Keller, 1992, "Neutron resonance spin echo-a new tool for high resolution spectroscopy," Physica B (Amsterdam) 180-181, 899-902.

Gammel, P. L., D. J. Bishop, M. R. Eskildsen, K. Mortensen, N. H. Andersen, I. R. Fisher, K. O. Cheon, P. C. Canfield, and V. G. Kogan, 1999, "Systematic studies of the square-hexagonal flux line lattice transition in $\mathrm{Lu}\left(\mathrm{Ni}_{1-x} \mathrm{Co}_{x}\right)_{2} \mathrm{~B}_{2} \mathrm{C}$ : The role of nonlocality," Phys. Rev. Lett. 82, 4082-4085.

Gammel, P. L., D. A. Huse, R. N. Kleiman, B. Batlogg, C. S. Oglesby, E. Bucher, D. J. Bishop, T. E. Mason, and K. Mortensen, 1994, "Small Angle Neutron Scattering Study of the Magnetic Flux-Line Lattice in Single Crystal $2 \mathrm{H}-\mathrm{NbSe}_{2}$," Phys. Rev. Lett. 72, 278-281.

Gammel, P. L., et al., 1998, "Structure and Correlations of the Flux Line Lattice in Crystalline Nb through the Peak Effect," Phys. Rev. Lett. 80, 833-836.

Gannon, W. J., W. P. Halperin, C. Rastovski, K. J. Schlesinger, J. Hlevyack, M. R. Eskildsen, A. B. Vorontsov, J. Gavilano, U. Gasser, and G. Nagy, 2015, "Nodal gap structure and order parameter symmetry of the unconventional superconductor $\mathrm{UPt}_{3}$, , New J. Phys. 17, 023041.

Garcia-Calderón, R. G., L. Fernández Barquín, S. N. Kaul, J. C. Gómez Sal, P. Gorria, J. S. Pedersen, and R. K. Heenan, 2005, "Small-angle neutron scattering study of a magnetically inhomogeneous amorphous alloy with reentrant behavior," Phys. Rev. B 71, 134413.

Gatel, C., F. J. Bonilla, A. Meffre, E. Snoeck, B. Warot-Fonrose, B. Chaudret, L.-M. Lacroix, and T. Blon, 2015, "Size-Specific Spin Configurations in Single Iron Nanomagnet: From Flower to Exotic Vortices," Nano Lett. 15, 6952-6957.

Gazeau, F., E. Dubois, J.-C. Bacri, F. Boué, A. Cebers, and R. Perzynski, 2002, "Anisotropy of the structure factor of magnetic fluids under a field probed by small-angle neutron scattering," Phys. Rev. E 65, 031403.

Gazquez, J., Shameek Bose, M. Sharma, M. A. Torija, S. J. Pennycook, C. Leighton, and M. Varela, 2013, "Lattice mismatch 
accommodation via oxygen vacancy ordering in epitaxial $\mathrm{La}_{0.5} \mathrm{Sr}_{0.5} \mathrm{CoO}_{3-\delta}$ thin films," APL Mater. 1, 012105.

Gerold, V., and G. Kostorz, 1978, "Small-angle scattering applications to materials science," J. Appl. Crystallogr. 11, 376-404.

Giblin, S. R., I. Terry, S. J. Clark, T. Prokscha, D. Prabhakaran, A. T. Boothroyd, J. Wu, and C. Leighton, 2005, "Observation of magnetic excitons in $\mathrm{LaCoO}_{3}$," Europhys. Lett. 70, 677.

Gilardi, R., J. Mesot, S. P. Brown, E. M. Forgan, S. L. Lee, R. Cubitt, C. D. Dewhurst, T. Uefuji, and K. Yamada, 2004, "Square vortex lattice at anomalously low magnetic fields in electron-doped $\mathrm{Nd}_{1.85} \mathrm{Ce}_{0.15} \mathrm{CuO}_{4}$," Phys. Rev. Lett. 93, 217001.

Gille, W., 2014, Particle and Particle Systems Characterization: Small-Angle Scattering (SAS) Applications (CRC Press, Boca Raton, FL).

Glasbrenner, J. K., K. M. Bussmann, and I. I. Mazin, 2014, "Magnetic spiral induced by strong correlations in $\mathrm{MnAu}_{2}$, , Phys. Rev. B 90, 144421.

Glatter, O., and O. Kratky, 1982, Eds., Small Angle X-ray Scattering (Academic Press, New York).

Glinka, C. J., J. G. Barker, B. Hammouda, S. Krueger, J. J. Moyer, and W. J. Orts, 1998, "The $30 \mathrm{~m}$ Small-Angle Neutron Scattering Instruments at the National Institute of Standards and Technology," J. Appl. Crystallogr. 31, 430-445.

Göbel, Börge, Alexander Mook, Jürgen Henk, and Ingrid Mertig, 2017, "Antiferromagnetic skyrmion crystals: Generation, topological Hall, and topological spin Hall effect," Phys. Rev. B 96, 060406.

Golosovsky, I. V., I. Mirebeau, G. André, D. A. Kurdyukov, Y. Kumzerov, and S. B. Vakhrushev, 2001, "Magnetic ordering and phase transition in $\mathrm{MnO}$ embedded in a porous glass," Phys. Rev. Lett. 86, 5783-5786.

Gopalakrishnan, Sarang, Benjamin L. Lev, and Paul M. Goldbart, 2009, "Emergent crystallinity and frustration with Bose-Einstein condensates in multimode cavities," Nat. Phys. 5, 845-850.

Gorn, N.L., D. V. Berkov, P. Görnert, and D. Stock, 2007, "Comparison of the Fast Multipole and Ewald methods for the evaluation of the magnetodipolar field in disordered systems," J. Magn. Magn. Mater. 310, 2829.

Griffiths, Robert B., 1969, "Nonanalytic behavior above the critical point in a random Ising ferromagnet," Phys. Rev. Lett. 23, 17-19.

Grigoriev, S., S. V. Maleyev, A. I. Okorokov, Yu. O. Chetverikov, and H. Eckerlebe, 2007, "Field-induced reorientation of helix in MnSi near $\mathrm{T}_{c}$," J. Magn. Magn. Mater. 310, 1599-1601.

Grigoriev, S. V., S. V. Maleyev, A. I. Okorokov, Yu. O. Chetverikov, P. Böni, R. Georgii, D. Lamago, H. Eckerlebe, and K. Pranzas, 2006a, "Magnetic structure of MnSi under an applied field probed by polarized small-angle neutron scattering," Phys. Rev. B 74, 214414.

Grigoriev, S. V., S. V. Maleyev, A. I. Okorokov, Yu. O. Chetverikov, and H. Eckerlebe, 2006b, "Field-induced reorientation of the spin helix in MnSi near $\mathrm{T}_{c}$," Phys. Rev. B 73, 224440.

Grigoriev, S. V., S. V. Maleyev, A. I. Okorokov, Yu. O. Chetverikov, R. Georgii, P. Böni, D. Lamago, H. Eckerlebe, and K. Pranzas, 2005, "Critical fluctuations in MnSi near $\mathrm{T}_{C}$ : A polarized neutron scattering study," Phys. Rev. B 72, 134420.

Grigoriev, S. V., A. S. Sukhanov, E. V. Altynbaev, S.-A. Siegfried, A. Heinemann, P. Kizhe, and S. V. Maleyev, 2015, "Spin waves in fullpolarized state of Dzyaloshinskii-Moriya helimagnets: Small-angle neutron scattering study," Phys. Rev. B 92, 220415(R).

Grigoriev, S. V., A. V. Syromyatnikov, A. P. Chumakov, N. A. Grigoryeva, K. S. Napolskii, I. V. Roslyakov, A. A. Eliseev, A. V.
Petukhov, and H. Eckerlebe, 2010, "Nanostructures: Scattering beyond the born approximation," Phys. Rev. B 81, 125405.

Grigoriev, S. V., et al., 2013, "Chiral properties of structure and magnetism in $\mathrm{Mn}_{1-x} \mathrm{Fe}_{x} \mathrm{Ge}$ compounds: When the left and the right are fighting, who wins?" Phys. Rev. Lett. 110, 207201.

Grigoriev, S. V., et al., 2014, "Flip of spin helix chirality and ferromagnetic state in $\mathrm{Fe}_{1-x} \mathrm{Co}_{x} \mathrm{Ge}$ compounds," Phys. Rev. B 90, 174414.

Grigoryeva, N. A., S. V. Grigoriev, H. Eckerlebe, A. A. Eliseev, A. V. Lukashin, and K.S. Napolskii, 2007, "Polarized small-angle neutron scattering study of two-dimensional spatially ordered systems of nickel nanowires," J. Appl. Crystallogr. 40, s532-s536.

Gross, F., B. S. Chandrasekhar, D. Einzel, K. Andres, P. J. Hirschfeld, H. R. Ott, J. Beuers, Z. Fisk, and J. L. Smith, 1986, "Anomalous Temperature-Dependence of the Magnetic-Field Penetration Depth in Superconducting UBe $_{13}$, , Z. Phys. B 64, 175-188.

Grutter, A. J., K. L. Krycka, E. V. Tartakovskaya, J. A. Borchers, K. S. M. Reddy, E. Ortega, A. Ponce, and B. J. H. Stadler, 2017, "Complex three-dimensional magnetic ordering in segmented nanowire arrays," ACS Nano 11, 8311-8319.

Guinier, A., 1994, X-Ray Diffraction in Crystals, Imperfect Crystals, and Amorphous Bodies (Dover, New York).

Guinier, A., and G. Fournet, 1955, Small-Angle Scattering of X-rays (Wiley, New York).

Gulec, A., D. Phelan, C. Leighton, and R. F. Klie, 2016, "Simultaneous First-Order Valence and Oxygen Vacancy Order/Disorder Transitions in $\left(\operatorname{Pr}_{0.85} \mathrm{Y}_{0.15}\right)_{0.7} \mathrm{Ca}_{0.3} \mathrm{CoO}_{3-\delta}$ via Analytical Transmission Electron Microscopy," ACS Nano 10, 938-947.

Günther, A., D. Honecker, J.-P. Bick, P. Szary, C. D. Dewhurst, U. Keiderling, A. V. Feoktystov, A. Tschöpe, R. Birringer, and A. Michels, 2014, "Magnetic field dependent small-angle neutron scattering on a Co nanorod array: evidence for intraparticle spin misalignment," J. Appl. Crystallogr. 47, 992-998.

Gurevich, A., and V. G. Kogan, 2001, "Effect of Fluctuations on Vortex Lattice Structural Transitions in Superconductors," Phys. Rev. Lett. 87, 177009.

Gutfleisch, O., M. A. Willard, E. Brück, C. H. Chen, S. G. Sankar, and J. Ping Liu, 2011, "Magnetic materials and devices for the 21st century: Stronger, lighter, and more energy efficient," Adv. Mater. 23, 821 .

Halpern, O., and M. H. Johnson, 1939, "On the Magnetic Scattering of Neutrons," Phys. Rev. 55, 898.

Hammouda, B., 2010, "SANS from Polymers-Review of the Recent Literature," Polym. Rev. 50, 14-39.

Hank, P., W. Besenböck, R. Gähler, and M. Köppe, 1997, “Zero-field neutron spin echo techniques for incoherent scattering," Physica B (Amsterdam) 234-236, 1130-1132.

Hansen, P. A., 1977, Risoe Report (thesis), Risoe National Laboratory, Gjellerup, Copenhagen No. 360, p. 69.

Hanson, H. A., X. Wang, I. K. Dimitrov, J. Shi, X. S. Ling, B. B. Maranville, C. F. Majkrzak, M. Laver, U. Keiderling, and M. Russina, 2011, "Structural evidence for an edge-contaminated vortex phase in a $\mathrm{Nb}$ crystal using neutron diffraction," Phys. Rev. B 84, 014506.

Hao, Z., J. R. Clem, M. W. McElfresh, L. Civale, A. P. Malozemoff, and F. Holtzberg, 1991, "Model for the Reversible Magnetization of High- $\kappa$ Type-II Superconductors-Application to High- $T_{c}$ Superconductors," Phys. Rev. B 43, 2844-2852.

Haslbeck, F., et al., 2019, "Ultrahigh-resolution neutron spectroscopy of low-energy spin dynamics in $\mathrm{UGe}_{2}$," Phys. Rev. B 99, 014429. 
Haverkort, M. W., et al., 2006, "Spin State Transition in $\mathrm{LaCoO}_{3}$ Studied Using Soft X-ray Absorption Spectroscopy and Magnetic Circular Dichroism,” Phys. Rev. Lett. 97, 176405.

Hayami, Satoru, Ryo Ozawa, and Yukitoshi Motome, 2017, "Effective bilinear-biquadratic model for noncoplanar ordering in itinerant magnets," Phys. Rev. B 95, 224424.

Hayter, J. B., 1988, "Interparticle interactions and polarization effects in colloids," J. Appl. Crystallogr. 21, 737-743.

He, C., S. El-Khatib, J. Wu, J. W. Lynn, H. Zheng, J. F. Mitchell, and C. Leighton, 2009, "Doping fluctuation-driven magneto-electronic phase separation in $\mathrm{La}_{1-x} \mathrm{Sr}_{x} \mathrm{CoO}_{3}$ single crystals," Europhys. Lett. 87, 27006.

He, C., M. A. Torija, J. Wu, J. W. Lynn, H. Zheng, J. F. Mitchell, and C. Leighton, 2007, "Non-Griffiths-like clustered phase above the Curie temperature of the doped perovskite cobaltite $\mathrm{La}_{1-x} \mathrm{Sr}_{x} \mathrm{CoO}_{3}$," Phys. Rev. B 76, 014401.

He, S. M., N. H. van Dijk, M. Paladugu, H. Schut, J. Kohlbrecher, F. D. Tichelaar, and S. van der Zwaag, 2010, "In situ determination of aging precipitation in deformed $\mathrm{Fe}-\mathrm{Cu}$ and $\mathrm{Fe}-\mathrm{Cu}-\mathrm{B}-\mathrm{N}$ alloys by time-resolved small-angle neutron scattering," Phys. Rev. B 82, 174111.

Heinemann, A., H. Hermann, A. Wiedenmann, N. Mattern, and K. Wetzig, 2000, "A small-angle neutron scattering model for polydisperse spherical particles with diffusion zones and application to soft magnetic metallic glass," J. Appl. Crystallogr. 33, 1386.

Heinemann, A., A. Wiedenmann, and M. Kammel, 2004, "The full potential of small-angle scattering with polarised neutrons for structure parameter evaluation in magnetic liquids," Physica B (Amsterdam) 350, E207.

Heinemann, A., A. Wiedenmann, M. Kammel, H. Bönnemann, and N. Matoussevitch, 2004, "High-quality structure parameter for magnetic liquids obtained by small-angle scattering of polarized neutrons," Appl. Organomet. Chem. 18, 561-564.

Heinemann, André, and Albrecht Wiedenmann, 2003, "Benefits of polarized small-angle neutron scattering on magnetic nanometer scale structure modeling," J. Appl. Crystallogr. 36, 845-849.

Heinemann, André, Albrecht Wiedenmann, and Martin Kammel, 2007, "Reordering of magnetic colloid structures in external magnetic fields," J. Appl. Crystallogr. 40, s57.

Heinze, S., K. von Bergmann, M. Menzel, J. Brede, A. Kubetzka, R. Wiesendanger, G. Bihlmayer, and S. Blügel, 2011, "Spontaneous atomic-scale magnetic skyrmion lattice in two dimensions," Nat. Phys. 7, 713-718.

Hejtmanek, J., E. Santava, K. Knizek, M. Marysko, Z. Jirák, T. Naito, H. Sasaki, and H. Fujishiro, 2010, "Metal-insulator transition and the $\mathrm{Pr}^{3+} / \mathrm{Pr}^{4+}$ valence shift in $\left(\operatorname{Pr}_{1-y} \mathrm{Y}_{y}\right)_{0.7} \mathrm{Ca}_{0.3} \mathrm{CoO}_{3}$," Phys. Rev. B 82, 165107.

Hellman, F., et al., 2017, "Interface-induced phenomena in magnetism,” Rev. Mod. Phys. 89, 025006.

Hennion, M., F. Moussa, G. Biotteau, J. Rodríguez-Carvajal, L. Pinsard, and A. Revcolevschi, 1998, "Liquidlike Spatial Distribution of Magnetic Droplets Revealed by Neutron Scattering in $\mathrm{La}_{1-x} \mathrm{Ca}_{x} \mathrm{MnO}_{3}$," Phys. Rev. Lett. 81, 1957-1960.

Herlitschke, M., S. Disch, I. Sergueev, K. Schlage, E. Wetterskog, L. Bergström, and R.P. Hermann, 2016, "Spin disorder in maghemite nanoparticles investigated using polarized neutrons and nuclear resonant scattering," J. Phys. Conf. Ser. 711, 012002.

Herpin, A., and P. Meriel, 1961, "Etude de l'antiferromagnetisme helicoidal de $\mathrm{MnAu}_{2}$ par diffraction de neutrons," J. Phys. Radium 22, 337-348.

Hicks, T. J., 1995, Magnetism in Disorder (Clarendon Press, Oxford)
Higgins, J. S., and R. S. Stein, 1978, "Recent developments in polymer applications of small-angle neutron, X-ray and light scattering,” J. Appl. Crystallogr. 11, 346-375.

Hilger, I., F. Bergner, A. Ulbricht, A. Wagner, T. Weissgärber, B. Kieback, C. Heintze, and C. D. Dewhurst, 2016, "Investigation of spark plasma sintered oxide-dispersion strengthened steels by means of small-angle neutron scattering," J. Alloys Compd. 685, 927-935.

Hirano, T., K. Takamori, M. Ichioka, and K. Machida, 2013, "Rotation of Triangular Vortex Lattice in the Two-Band Superconductor $\mathrm{MgB}_{2}$," J. Phys. Soc. Jpn. 82, 063708.

Hoell, A., R. Müller, A. Wiedenmann, and W. Gawalek, 2002, "Core-shell and magnetic structure of barium hexaferrite fluids studied by SANS," J. Magn. Magn. Mater. 252, 92-94.

Hoell, A., A. Wiedenmann, U. Heyen, and D. Schüler, 2004, "Nanostructure and field-induced arrangement of magnetosomes studied by SANSPOL," Physica B (Amsterdam) 350, E309.

Hollamby, M. J., 2013, "Practical applications of small-angle neutron scattering," Phys. Chem. Chem. Phys. 15, 10566-10579.

Honecker, D., C. D. Dewhurst, K. Suzuki, S. Erokhin, and A. Michels, 2013, "Analysis of magnetic neutron-scattering data of two-phase ferromagnets," Phys. Rev. B 88, 094428.

Honecker, D., A. Ferdinand, F. Döbrich, C. D. Dewhurst, A. Wiedenmann, C. Gómez-Polo, K. Suzuki, and A. Michels, 2010, "Longitudinal polarization analysis in small-angle neutron scattering," Eur. Phys. J. B 76, 209-213.

Honecker, D., and A. Michels, 2013, "Theory of magnetic smallangle neutron scattering of two-phase ferromagnets," Phys. Rev. B 87, 224426.

Hono, K., and H. Sepehri-Amin, 2012, "Strategy for high-coercivity $\mathrm{Nd}-\mathrm{Fe}-\mathrm{B}$ magnets," Scr. Mater. 67, 530.

Hsieh, D., D. Quian, L. Wray, Y. Xia, Y. Hor, R. Cava, and M. Hasan, 2008, "A topological Dirac insulator in a quantum spin Hall phase," Nature (London) 452, 970.

Hubert, A., and R. Schäfer, 1998, Magnetic Domains (Springer, Berlin).

Huebener, R. P., 2001, Magnetic Flux Structures in Superconductors (Springer, New York).

Huxley, A., P. Rodière, D. McK. Paul, N. van Dijk, R. Cubitt, and J. Flouquet, 2000, "Realignment of the flux-line lattice by a change in the symmetry of superconductivity in $\mathrm{UPt}_{3}$," Nature (London) 406, 160-164.

Hyde, J. M., M. G. Burke, G. D. W. Smith, P. Styman, H. Swan, and K. Wilford, 2014, "Uncertainties and assumptions associated with APT and SANS characterisation of irradiation damage in RPV steels," J. Nucl. Mater. 449, 308-314.

Ibel, K., 1976, "The neutron small-angle camera D11 at the high-flux reactor, Grenoble," J. Appl. Crystallogr. 9, 296-309.

Ichioka, M., H. Adachi, T. Mizushima, and K. Machida, 2007, "Vortex state in a Fulde-Ferrell-Larkin-Ovchinnikov superconductor based on quasiclassical theory," Phys. Rev. B 76, 014503.

Ichioka, M., A. Hasegawa, and K. Machida, 1999, "Field dependence of the vortex structure in $d$-wave and $s$-wave superconductors," Phys. Rev. B 59, 8902-8916.

Ijiri, Y., C. V. Kelly, J. A. Borchers, J. J. Rhyne, D. F. Farrell, and S. A. Majetich, 2005, "Detection of spin coupling in iron nanoparticles with small angle neutron scattering," Appl. Phys. Lett. 86, 243102.

Imada, M., A. Fujimori, and Y. Tokura, 1998, "Metal-insulator transitions," Rev. Mod. Phys. 70, 1039-1263.

Inosov, D. S., et al., 2010a, "Symmetry and disorder of the vitreous vortex lattice in overdoped $\mathrm{BaFe}_{2-x} \mathrm{Co}_{x} \mathrm{As}_{2}$ : Indication for strong single-vortex pinning," Phys. Rev. B 81, 014513. 
Inosov, D. S., et al., 2010b, "Weak Superconducting Pairing and a Single Isotropic Energy Gap in Stoichiometric LiFeAs," Phys. Rev. Lett. 104, 187001.

Ishikawa, Y., and M. Arai, 1984, "Magnetic Phase Diagram of MnSi near Critical Temperature Studied by Neutron Small Angle Scattering,” J. Phys. Soc. Jpn. 53, 2726-2733.

Ishikawa, Y., K. Tajima, D. Bloch, and M. Roth, 1976, "Helical spin structure in manganese silicide MnSi," Solid State Commun. 19, 525 .

Ishiwata, S., M. Tokunaga, Y. Kaneko, D. Okuyama, Y. Tokunaga, S. Wakimoto, K. Kakurai, T. Arima, Y. Taguchi, and Y. Tokura, 2011, "Versatile helimagnetic phases under magnetic fields in cubic perovskite $\mathrm{SrFeO}_{3}$," Phys. Rev. B 84, 054427.

Izyumov, A. Yu., and S. V. Maleev, 1962, "Scattering of polarized neutrons by ferromagnets and antiferromagnets," Sov. Phys. JETP 14, 1168 [ZhETF 41, 1644 (1962)].

Jacrot, B., 1976, "The study of biological structures by neutron scattering from solution," Rep. Prog. Phys. 39, 911-953.

Jain, N., C. K. Liu, B. S. Hawkett, G. G. Warr, and W. A. Hamilton, 2014, "Application of small-angle neutron scattering to the study of forces between magnetically chained monodisperse ferrofluid emulsion droplets," J. Appl. Crystallogr. 47, 41-52.

Jaksch, S., 2019, private communication.

Jaksch, S., D. Martin-Rodriguez, A. Ostermann, J. Jestin, S. Duarte Pinto, W. G. Bouwman, J. Uher, R. Engels, and H. Frielinghaus, 2014, "Concept for a time-of-flight Small Angle Neutron Scattering instrument at the European Spallation Source," Nucl. Instrum. Methods Phys. Res., Sect. A 762, 22-30.

Janoschek, M., P. Fischer, J. Schefer, B. Roessli, V. Pomjakushin, M. Meven, V. Petricek, G. Petrakovskii, and L. Bezmaternikh, 2010, "Single magnetic chirality in the magnetoelectric $\mathrm{NdFe}_{3}\left({ }^{11} \mathrm{BO}_{3}\right)_{4}$," Phys. Rev. B 81, 094429.

Janoschek, M., M. Garst, A. Bauer, P. Krautscheid, R. Georgii, P. Böni, and C. Pfleiderer, 2013, "Fluctuation-induced first-order phase transition in dzyaloshinskii-moriya helimagnets," Phys. Rev. B 87, 134407.

Janoschek, M., S. Klimko, R. Gaehler, B. Roessli, and P. Boeni, 2007, "Spherical neutron polarimetry with MuPAD," Physica B (Amsterdam) 397, 125-130.

Jensen, J., and A. R. Mackintosh, 1990, Phys. Rev. Lett. 64, 2699. Jonietz, F., et al., 2010, "Spin transfer torques in MnSi at ultralow current densities," Science 330, 1648.

Jonker, G. H., and J.H. Van Santen, 1950, "Ferromagnetic compounds of manganese with perovskite structure," Physica (Utrecht) 16, 337-349.

Jonker, G. H., and J.H. Van Santen, 1953, "Magnetic compounds wtth perovskite structure III. ferromagnetic compounds of cobalt," Physica (Utrecht) 19, 120-130.

Joseph, Aswathy, and Suresh Mathew, 2014, "Ferrofluids: Synthetic Strategies, Stabilization, Physicochemical Features, Characterization, and Applications," ChemPlusChem 79, 1382-1420.

Joumard, I., J. Marcus, T. Klein, and R. Cubitt, 1999, "Small angle neutron scattering and magnetization measurements in the cubic (K, Ba) $\mathrm{BiO}_{3}$ superconductor," Phys. Rev. Lett. 82, 4930-4933.

Joynt, R., and L. Taillefer, 2002, "The superconducting phases of $\mathrm{UPt}_{3}$, , Rev. Mod. Phys. 74, 235.

Kampmann, R., and R. Wagner, 1986, "Phase transformations in Fe$\mathrm{Cu}$ alloys-SANS experiments and theory," in Atomic Transport and Defects in Metals by Neutron Scattering, edited by C. Janot, W. Petry, D. Richter, and T. Springer (Springer-Verlag, Berlin), pp. 73-77.

Karaca, H. E., I. Karaman, B. Basaran, Y. Ren, Y. I. Chumlyakov, and H. J. Maier, 2009, "Magnetic Field-Induced Phase Transformation in NiMnCoIn Magnetic Shape-Memory Alloys-A New Actuation Mechanism with Large Work Output," Adv. Funct. Mater. 19, 983-998.

Karge, Lukas, Ralph Gilles, and Sebastian Busch, 2017, "Calibrating SANS data for instrument geometry and pixel sensitivity effects: access to an extended $Q$ range," J. Appl. Crystallogr. 50, 1382-1394.

Karhu, E. A., U. K. Rößler, A. N. Bogdanov, S. Kahwaji, B. J. Kirby, H. Fritzsche, M. D. Robertson, C.F. Majkrzak, and T. L. Monchesky, 2012, "Chiral modulations and reorientation effects in MnSi thin films," Phys. Rev. B 85, 094429.

Karube, K., et al., 2016, "Robust metastable skyrmions and their triangular-square lattice structural transition in a high-temperature chiral magnet," Nat. Mater. 15, 1237-1242.

Kasuya, T., 1956, "A Theory of Metallic Ferro- and Antiferromagnetism on Zener's Model," Prog. Theor. Phys. 16, 45.

Katano, S., and M. Iizumi, 1984, "Crossover phenomenon in dynamical scaling of phase separation in Fe-Cr alloy," Phys. Rev. Lett. 52, 835-838.

Kataoka, M., 1987, "Spin waves in systems with long period helical spin density waves due to the antisymmetric and symmetric exchange interactions," J. Phys. Soc. Jpn. 56, 3635-3647.

Katsura, H., N. Nagaosa, and A. V. Balatsky, 2005, "Spin Current and Magnetoelectric Effect in Noncollinear Magnets," Phys. Rev. Lett. 95, 057205.

Kawamura, H., 1998, "Universality of phase transitions of frustrated antiferromagnets," J. Phys. Condens. Matter 10, 4707-4754.

Kawano-Furukawa, H., et al., 2011, "Gap in $\mathrm{KFe}_{2} \mathrm{As}_{2}$ studied by small-angle neutron scattering observations of the magnetic vortex lattice," Phys. Rev. B 84, 024507.

Kawano-Furukawa, H., et al., 2013, "Probing the anisotropic vortex lattice in the Fe-based superconductor $\mathrm{KFe}_{2} \mathrm{As}_{2}$ using small-angle neutron scattering," Phys. Rev. B 88, 134524.

Kealey, P. G., et al., 2000, "Reconstruction from Small-Angle Neutron Scattering Measurements of the Real Space Magnetic Field Distribution in the Mixed State of $\mathrm{Sr}_{2} \mathrm{RuO}_{4}$," Phys. Rev. Lett. 84, 6094-6097.

Kealey, P. G., et al., 2001, "Transverse-field components of the fluxline lattice in the anisotropic superconductor $\mathrm{YBa}_{2} \mathrm{Cu}_{3} \mathrm{O}_{7-\delta}$, " Phys. Rev. B 64, 174501.

Keiderling, U., 2002, "The new 'BerSANS-PC' software for reduction and treatment of small angle neutron scattering data," Appl. Phys. A 74, s1455.

Keiderling, U., A. Wiedenmann, A. Rupp, J. Klenke, and W. Heil, 2008, "SANS polarization analysis at V4 SANS instrument of HMI Berlin,” Meas. Sci. Technol. 19, 034009.

Keimer, B., F. Dogan, I. A. Aksay, R. W. Erwin, J. W. Lynn, and M. Sarikaya, 1993, "Inclined-Field Structure, Morphology, and Pinning of the Vortex Lattice in Microtwinned $\mathrm{YBa}_{2} \mathrm{Cu}_{3} \mathrm{O}_{7}$," Science 262, 83-86.

Keimer, B., W. Y. Shih, R. W. Erwin, J. W. Lynn, F. Dogan, and I. A. Aksay, 1994, "Vortex Lattice Symmetry and Electronic Structure in $\mathrm{YBa}_{2} \mathrm{Cu}_{3} \mathrm{O}_{7}$," Phys. Rev. Lett. 73, 3459-3462.

Keller, T., T. Krist, A. Danzig, U. Keiderling, F. Mezei, and A. Wiedenmann, 2000, "The polarized neutron small-angle scattering instrument at BENSC Berlin," Nucl. Instrum. Methods Phys. Res., Sect. A 451, 474.

Kemoklidze, M.P., 1965, "Scattering of neutrons by quantized magnetic flux lines in type II superconductors," Sov. Phys. JETP 20, 1505-1507.

Kenzelmann, M., et al., 2005, "Magnetic Inversion Symmetry Breaking and Ferroelectricity in $\mathrm{TbMnO}_{3}$," Phys. Rev. Lett. 95, 087206. 
Kézsmárki, I., et al., 2015, "Néel-type skyrmion lattice with confined orientation in the polar magnetic semiconductor $\mathrm{GaV}_{4} \mathrm{~S}_{8}$," Nat. Mater. 14, 1116-1122.

Kimura, T., 2007, "Spiral Magnets as Magnetoelectrics," Annu. Rev. Mater. Res. 37, 387-413.

Kimura, T., 2012, "Magnetoelectric Hexaferrites," Annu. Rev. Condens. Matter Phys. 3, 93-110.

Kimura, T., T. Goto, H. Shintani, K. Ishizaka, T. Arima, and Y. Tokura, 2003, "Magnetic control of ferroelectric polarization," Nature (London) 426, 55-58.

Kimura, T., G. Lawes, and A. P. Ramirez, 2005, "Electric polarization rotation in a hexaferrite with long-wavelength magnetic structures," Phys. Rev. Lett. 94, 137201.

Kindervater, J., W. Häußler, M. Janoschek, C. Pfleiderer, P. Böni, and M. Garst, 2014, "Critical spin-flip scattering at the helimagnetic transition of MnSi,” Phys. Rev. B 89, 180408(R).

Kindervater, J., S. Säubert, and P. Böni, 2017, "Dipolar effects on the critical fluctuations in Fe: Investigation by the neutron spin-echo technique MIEZE," Phys. Rev. B 95, 014429.

Kindervater, J., et al., 2018, "Evolution of magnetocrystalline anisotropies in $\mathrm{Mn}_{1-x} \mathrm{Fe}_{x} \mathrm{Si}$ and $\mathrm{Mn}_{1-x} \mathrm{Co}_{x} \mathrm{Si}$ as observed by small-angle neutron scattering," arXiv:1811.12379.

Klein, T., I. Joumard, S. Blanchard, J. Marcus, R. Cubitt, T. Giamarchi, and P. Le Doussal, 2001, "A Bragg glass phase in the vortex lattice of a type II superconductor," Nature (London) 413, 404-406.

Kleiner, W. H., S. H. Autler, and L. M. Roth, 1964, "Bulk Solution of Ginzburg-Landau Equations for Type 2 Superconductors-Upper Critical Field Region,” Phys. Rev. 133, A1226.

Kline, S. R., 2006, "Reduction and analysis of SANS and USANS data using IGOR Pro," J. Appl. Crystallogr. 39, 895.

Klokkenburg, M., B. H. Erné, A. Wiedenmann, A. V. Petukhov, and A. P. Philipse, 2007, "Dipolar structures in magnetite ferrofluids studied with small-angle neutron scattering with and without applied magnetic field," Phys. Rev. E 75, 051408.

Knaster, J., A. Moeslang, and T. Muroga, 2016, "Materials research for fusion," Nat. Phys. 12, 424-434.

Koblischka, M. R., and U. Hartmann, 2003, "Recent advances in magnetic force microscopy," Ultramicroscopy 97, 103-112.

Kodama, R., and A. Berkowitz, 1999, "Atomic-scale magnetic modeling of oxide nanoparticles," Phys. Rev. B 59, 6321-6336.

Kodama, R. H., A.E. Berkowitz, E. J. McNiff, Jr., and S. Foner, 1996, "Surface Spin Disorder in $\mathrm{NiFe}_{2} \mathrm{O}_{4}$ Nanoparticles," Phys. Rev. Lett. 77, 394-397.

Kogan, V. G., M. Bullock, B. Harmon, P. Miranović, Lj. Dobrosavlević-Grujić, P. L. Gammel, and D. J. Bishop, 1997, "Vortex lattice transitions in borocarbides," Phys. Rev. B 55, R8693-R8696.

Kohlbrecher, J., and W. Wagner, 2000, "The new SANS instrument at the Swiss spallation source SINQ," J. Appl. Crystallogr. 33, 804-806.

Konig, M., S. Wiedmann, C. Brune, A. Roth, H. Buhmann, L. Molenkamp, X.-L. Qi, and S.-C. Zhang, 2007, "Quantum Spin Hall Insulator State in HgTe Quantum Wells," Science 318, 766.

Koretsune, T., N. Nagaosa, and R. Arita, 2015, "Control of Dzyaloshinskii-Moriya interaction in $\mathrm{Mn}_{1-x} \mathrm{Fe}_{x} \mathrm{Ge}$ : a firstprinciples study," Sci. Rep. 5, 13302.

Korotin, M. A., S. Yu. Ezhov, I. V. Solovyev, V. I. Anisimov, D. I. Khomskii, and G. A. Sawatzky, 1996, "Intermediate-spin state and properties of $\mathrm{LaCoO}_{3}$," Phys. Rev. B 54, 5309-5316.

Kostorz, G., 1982, in Small Angle X-ray Scattering, edited by O. Glatter and O. Kratky (Academic Press, New York), pp. 467-498.
Kostorz, G., 1991, "Small-angle scattering studies of phase separation and defects in inorganic materials," J. Appl. Crystallogr. 24, 444-456.

Kostorz, G., 2014, "X-ray and Neutron Scattering," in Physical Metallurgy, edited by D. E. Laughlin and K. Hono (Elsevier, New York), pp. 1227-1316, 5th ed.

Kosugi, T., S. Kawano, N. Achiwa, A. Onodera, Y. Nakai, and N. Yamamoto, 2003, "Direct evidence of helifan structures in holmium by single crystal neutron diffraction," Physica B (Amsterdam) 334, 365-368.

Kovács, András, Kazuhisa Sato, Vlado K. Lazarov, Pedro L. Galindo, Toyohiko J. Konno, and Yoshihiko Hirotsu, 2009, "Direct observation of a surface induced disordering process in magnetic nanoparticles,” Phys. Rev. Lett. 103, 115703.

Kozlenko, D. P., N. O. Golosova, Z. Jirák, L. S. Dubrovinsky, B. N. Savenko, M. G. Tucker, Y. Le Godec, and V. P. Glazkov, 2007, "Temperature- and pressure-driven spin-state transitions in $\mathrm{LaCoO}_{3}$," Phys. Rev. B 75, 064422.

Krenke, T., M. Acet, E. F. Wassermann, X. Moya, L. Mañosa, and A. Planes, 2005, "Martensitic transitions and the nature of ferromagnetism in the austenitic and martensitic states of Ni-Mn-Sn alloys," Phys. Rev. B 72, 014412.

Kreyssig, A., R. Prozorov, C. D. Dewhurst, P. C. Canfield, R. W. McCallum, and A. I. Goldman, 2009, "Probing fractal magnetic domains on multiple length scales in $\mathrm{Nd}_{2} \mathrm{Fe}_{14} \mathrm{~B}$," Phys. Rev. Lett. 102, 047204.

Kronmüller, H., and M. Fähnle, 2003, Micromagnetism and the Microstructure of Ferromagnetic Solids (Cambridge University Press, Cambridge, England).

Kronmüller, H., A. Seeger, and M. Wilkens, 1963, "Depolarisation und Kleinwinkelstreuung von Neutronen durch Gitterfehler in ferromagnetischen Kristallen,” Z. Phys. 171, 291-311.

Krycka, K., W. Chen, J. Borchers, B. Maranville, and S. Watson, 2012, "Polarization-analyzed small-angle neutron scattering. I. Polarized data reduction using Pol-Corr," J. Appl. Crystallogr. 45, 546-553.

Krycka, K. L., J. A. Borchers, R. A. Booth, Y. Ijiri, K. Hasz, J. J. Rhyne, and S. A. Majetich, 2014, "Origin of surface canting within $\mathrm{Fe}_{3} \mathrm{O}_{4}$ nanoparticles," Phys. Rev. Lett. 113, 147203.

Krycka, K. L., J. A. Borchers, R. A. Booth, Y. Ijiri, K. Hasz, J. J. Rhyne, and S. A. Majetich, 2015, "Krycka et al. Reply:," Phys. Rev. Lett. 114, 149702.

Krycka, K. L., et al., 2010a, "Core-Shell Magnetic Morphology of Structurally Uniform Magnetite Nanoparticles," Phys. Rev. Lett. 104, 207203.

Krycka, K. L., et al., 2010b, "Internal magnetic structure of magnetite nanoparticles at low temperature," J. Appl. Phys. 107, 09B525.

Krycka, K., J. Borchers, Y. Ijiri, R. Booth, and S. Majetich, 2012, "Polarization-analyzed small-angle neutron scattering. II. Mathematical angular analysis," J. Appl. Crystallogr. 45, 554-565.

Kugel, K. I., A. L. Rakhmanov, and A. O. Sboychakov, 2005, "Phase Separation in Jahn-Teller Systems with Localized and Itinerant Electrons," Phys. Rev. Lett. 95, 267210.

Kugler, M., et al., 2015, "Band structure of helimagnons in $\mathrm{MnSi}$ resolved by inelastic neutron scattering," Phys. Rev. Lett. 115, 097203.

Kuhn, S. J., et al., 2016, "Simultaneous evidence for Pauli paramagnetic effects and multiband superconductivity in $\mathrm{KFe}_{2} \mathrm{As}_{2}$ by small-angle neutron scattering studies of the vortex lattice," Phys. Rev. B 93, 104527.

Kuhn, S. J., et al., 2017, "Anisotropy and multiband superconductivity in $\mathrm{Sr}_{2} \mathrm{RuO}_{4}$ determined by small-angle neutron scattering studies of the vortex lattice," Phys. Rev. B 96, 174507. 
Kuhns, P. L., M. J. R. Hoch, W. G. Moulton, A. P. Reyes, J. Wu, and C. Leighton, 2003, "Magnetic phase separation in $\mathrm{La}_{1-x} \mathrm{Sr}_{x} \mathrm{CoO}_{3}$ by ${ }^{59}$ Co nuclear magnetic resonance," Phys. Rev. Lett. 91, 127202.

Kurimský, J., M. Raják, R. Cimbala, K. Paulovičová, M. Timko, P. Kopčanský, M. Kolcun, M. Kosterec, and L. Kruželák, 2017, "Generation of $\mathrm{Fe}_{3} \mathrm{O}_{4}$ Nanoparticle Aggregates in a Ferrofluid Driven by External Electric Field," Acta Phys. Pol. A 131, 907-909.

Kurumaji, T., T. Nakajima, V. Ukleev, A. Feoktystov, T. Arima, K. Kakurai, and Y. Tokura, 2017, "Neel-type skyrmion lattice in tetragonal polar magnet $\mathrm{VOSe}_{2} \mathrm{O}_{5}$," Phys. Rev. Lett. 119, 237201.

Landau, L., and E. Lifshitz, 1935, "On the theory of the dispersion of magnetic permeability in ferromagnetic bodies," Phys. Z. Sowjetunion 8, 153-169.

LaSalle, J. C., and L. H. Schwartz, 1986, "Further studies of spinodal decomposition in Fe-Cr," Acta Metall. 34, 989-1000.

Laver, M., 2012, "Small-angle scattering of nanostructures and nanomaterials," in Encyclopedia of Nanotechnology, edited by Bharat Bhushan (Springer Netherlands, Dordrecht), pp. 24372450.

Laver, M., and E. M. Forgan, 2010, "Magnetic flux lines in type-II superconductors and the 'hairy ball' theorem," Nat. Commun. 1, 1.

Laver, M., E. M. Forgan, A. B. Abrahamsen, C. Bowell, T. Geue, and R. Cubitt, 2008, "Uncovering Flux Line Correlations in Superconductors by Reverse Monte Carlo Refinement of Neutron Scattering Data," Phys. Rev. Lett. 100, 107001.

Laver, M., et al., 2006, "Spontaneous symmetry-breaking vortex lattice transitions in pure niobium," Phys. Rev. Lett. 96, 167002.

Laver, M., et al., 2009, "Structure and degeneracy of vortex lattice domains in pure superconducting niobium: A small-angle neutron scattering study," Phys. Rev. B 79, 014518.

Lawes, G., et al., 2005, "Magnetically Driven Ferroelectric Order in $\mathrm{Ni}_{3} \mathrm{~V}_{2} \mathrm{O}_{8}$," Phys. Rev. Lett. 95, 087205.

Lawler, M. J., H.-Y. Kee, Y. B. Kim, and A. Vishwanath, 2008, "Topological Spin Liquid on the Hyperkagome Lattice of $\mathrm{Na}_{4} \mathrm{Ir}_{3} \mathrm{O}_{8}$," Phys. Rev. Lett. 100, 227201.

Lebech, B., P. Harris, J. S. Pedersen, K. Mortensen, C. I. Gregory, N. R. Bernhoeft, M. Jermy, and S. A. Brown, 1995, "Magnetic phase diagram of MnSi," J. Magn. Magn. Mater. 140-144, 119-120.

Lee, Sung Ho, Dong Heon Lee, Hyun Jung, Young-Soo Han, Tae-Hwan Kim, and Woochul Yang, 2015, "Magnetic properties of $\mathrm{SiO}_{2}$-coated iron oxide nanoparticles studied by polarized small angle neutron scattering," Curr. Appl. Phys. 15, 915-919.

Lee, Y., and B. N. Harmon, 2013, "Rhombohedral distortion effects on electronic structure of $\mathrm{LaCoO}_{3}$," J. Appl. Phys. 113, 17E145.

Leibler, L., 1980, "Theory of microphase separation in block copolymers," Macromolecules 13, 1602-1617.

Leighton, C., et al., 2009, "Coupled structural/magnetocrystalline anisotropy transitions in the doped perovskite cobaltite $\mathrm{Pr}_{1-x} \mathrm{Sr}_{x} \mathrm{CoO}_{3}$," Phys. Rev. B 79, 214420.

Leonov, A. O., and M. Motovoy, 2015, "Multiply periodic states and isolated skyrmions in an anisotropic frustrated magne," Nat. Commun. 6, 8275.

Leos, N. G., et al., 2015, "Influence of the Fermi Surface Morphology on the Magnetic Field-Driven Vortex Lattice Structure Transitions in $\mathrm{YBa}_{2} \mathrm{Cu}_{3} \mathrm{O}_{7-\delta}: \delta=0,0.15$,' J. Phys. Soc. Jpn. 84, 044709.

Levett, S. J., C. D. Dewhurst, and D. McK. Paul, 2002, "Vortexlattice transitions in $\mathrm{YNi}_{2} \mathrm{~B}_{2} \mathrm{C}$ : Nature of the 45-degree reorientation,” Phys. Rev. B 66, 014515.

Ling, C. D., E. Granado, J. J. Neumeier, J. W. Lynn, and D. N. Argyriou, 2003, "Inhomogeneous magnetism in La-doped
$\mathrm{CaMnO}_{3}$. I. Mesoscopic phase separation due to lattice-coupled ferromagnetic interactions," Phys. Rev. B 68, 134439.

Lister, S. J., et al., 2009, "Small-angle polarized neutron studies of perpendicular magnetic recording media," J. Appl. Phys. 106, 063908.

Lister, S. J., et al., 2010, "Size-dependent reversal of grains in perpendicular magnetic recording media measured by small-angle polarized neutron scattering," Appl. Phys. Lett. 97, 112503.

Liu, J., H. Sepehri-Amin, T. Ohkubo, K. Hioki, A. Hattori, and K. Hono, 2014, "Microstructure evolution of hot-deformed Nd-Fe-B anisotropic magnets," J. Appl. Phys. 115, 17A744.

Liu, J., H. Sepehri-Amin, T. Ohkubo, K. Hioki, A. Hattori, T. Schrefl, and K. Hono, 2013, "Effect of Nd content on the microstructure and coercivity of hot-deformed Nd-Fe-B permanent magnets," Acta Mater. 61, 5387-5399.

Locatelli, A., and E. Bauer, 2008, "Recent advances in chemical and magnetic imaging of surfaces and interfaces by XPEEM," J. Phys. Condens. Matter 20, 093002.

Löffler, J. F., H. B. Braun, W. Wagner, G. Kostorz, and A. Wiedenmann, 2005, "Magnetization processes in nanostructured metals and smallangle neutron scattering," Phys. Rev. B 71, 134410.

Lonzarich, G. G., and L. Taillefer, 1985, "Effect of spin fluctuations on the magnetic equation of state of ferromagnetic or nearly ferromagnetic metals," J. Phys. C 18, 4339.

Louden, E. R., C. Rastovski, C. D. Dewhurst, N. D. Zhigadlo, and M. R. Eskildsen, 2018, unpublished.

Lovesey, S. W., 1984, Theory of Neutron Scattering from Condensed Matter, Vols. I and II (Clarendon Press, Oxford).

Lu, An Hui, E. L. Salabas, and Ferdi Schüth, 2007, "Magnetic nanoparticles: Synthesis, protection, functionalization, and application,” Angew. Chem., Int. Ed. Engl. 46, 1222-1244.

Lynn, J. W., R. W. Erwin, J. A. Borchers, Q. Huang, A. Santoro, J.-L. Peng, and Z. Y. Li, 1996, "Unconventional ferromagnetic transition in $\mathrm{La}_{1-x} \mathrm{Ca}_{x} \mathrm{MnO}_{3}$," Phys. Rev. Lett. 76, 4046-4049.

Lynn, J. W., L. Vasiliu-Doloc, and M. A. Subramanian, 1998, "Spin dynamics of the magnetoresistive pyrochlore $\mathrm{Tl}_{2} \mathrm{Mn}_{2} \mathrm{O}_{7}$," Phys. Rev. Lett. 80, 4582-4585.

Maeno, Y., S. Kittaka, T. Nomura, S. Yonezawa, and K. Ishida, 2012, "Evaluation of Spin-Triplet Superconductivity in $\mathrm{Sr}_{2} \mathrm{RuO}_{4}$," J. Phys. Soc. Jpn. 81, 011009.

Magen, C., P. A. Algarabel, L. Morellon, J. P. Araújo, C. Ritter, M. R. Ibarra, A. M. Pereira, and J. B. Sousa, 2006, "Observation of a griffiths-like phase in the magnetocaloric compound $\mathrm{Tb}_{5} \mathrm{Si}_{2} \mathrm{Ge}_{2}$," Phys. Rev. Lett. 96, 167201.

Mahendiran, R., A. Maignan, S. Hébert, C. Martin, M. Hervieu, B. Raveau, J. F. Mitchell, and P. Schiffer, 2002, "Ultrasharp magnetization steps in perovskite manganites," Phys. Rev. Lett. 89, 286602.

Mahendiran, R., and P. Schiffer, 2003, "Double magnetic transition in $\mathrm{Pr}_{0.5} \mathrm{Sr}_{0.5} \mathrm{CoO}_{3}$," Phys. Rev. B 68, 024427.

Makino, K., D. Okuyama, M. Avdeev, and K. Sato, 2016, "Incommensurate Magnetic Structure in the Cubic Noncentrosymmetric Ternary Compound $\operatorname{Pr}_{r} \mathrm{Ru}_{3} \mathrm{Al}_{2}$," J. Phys. Soc. Jpn. 85, 073705.

Makino, K., J. D. Reim, D. Higashi, D. Okuyama, T. J. Sato, Y. Nambu, E. P. Gilbert, N. Booth, S. Seki, and Y. Tokura, 2017, "Thermal stability and irreversibility of skyrmion-lattice phases in $\mathrm{Cu}_{2} \mathrm{OSeO}_{3}$," Phys. Rev. B 95, 134412.

Maleev, S. V., 1961, "Polarization resulting from scattering of neutrons by ferromagnetic substances," Sov. Phys. JETP 13, 860.

Maleev, S. V., 1965, "Inelastic small angle neutron scattering in ferromagnets," Sov. Phys. JETP 21, 969-975.

Maleev, S. V., V. G. Bar'yakhtar, and R. A. Suris, 1963, "The scattering of slow neutrons by complex magnetic structures," Sov. Phys. Solid State 4, 2533. 
Manna, P. K., S. M. Yusuf, M. D. Mukadam, and J. Kohlbrecher, 2012, "Evidence of a coreshell structure in the antiferromagnetic $\mathrm{La} 0.2 \mathrm{Ce} 0.8 \mathrm{CrO} 3$ nanoparticles by neutron scattering," Appl. Phys. A 109, 385-390.

Manton, N., and P. Sutcliffe, 2004, Topological Solitions (Cambridge University Press, Cambridge, England).

Manyala, N., Y. Sidis, J. F. Ditusa, G. Aeppli, D. P. Young, and Z. Fisk, 2004, "Large anomalous Hall effect in a silicon-based magnetic semiconductor," Nat. Mater. 3, 255-262.

Marcano, N., J. C. Gómez Sal, J. I. Espeso, J. M. De Teresa, P. A. Algarabel, C. Paulsen, and J. R. Iglesias, 2007, "Mesoscopic magnetic states in metallic alloys with strong electronic correlations: A percolative scenario for $\mathrm{CeNi}_{1-x} \mathrm{Cu}_{x}$, Phys. Rev. Lett. 98, 166406.

Marshall, W., and R. D. Lowde, 1968, "Magnetic correlations and neutron scattering," Rep. Prog. Phys. 31, 705.

Martin, J.E., and A. J. Hurd, 1987, "Scattering from fractals," J. Appl. Crystallogr. 20, 61-78.

Martin, N., 2018, "On the resolution of a MIEZE spectrometer," Nucl. Instrum. Methods Phys. Res., Sect. A 882, 11-16.

Martin, N., M. Deutsch, G. Chaboussant, F. Damay, P. Bonville, L. N. Fomicheva, A. V. Tsvyashchenko, U. K. Rössler, and I. Mirebeau, 2017, "Long-period helical structures and twist-grain boundary phases induced by chemical substitution in the $\mathrm{Mn}_{1-x}(\mathrm{Co}, \mathrm{Rh})_{x} \mathrm{Ge}$ chiral magnet," Phys. Rev. B 96, 020413.

Marziali Bermudez, M., M. R. Eskildsen, M. Bartkowiak, G. Nagy, V. Bekeris, and G. Pasquini, 2015, "Dynamic Reorganization of Vortex Matter into Partially Disordered Lattices," Phys. Rev. Lett. 115, 067001.

Marziali Bermudez, M., E. R. Louden, M. R. Eskildsen, C. D. Dewhurst, V. Bekeris, and G. Pasquini, 2017, "Metastability and hysteretic vortex pinning near the order-disorder transition in $\mathrm{NbSe}_{2}$ : An interplay between plastic and elastic energy barriers," Phys. Rev. B 95, 104505.

Mathon, M. H., Y. de Carlan, G. Geoffroy, X. Averty, A. Alamo, and C. H. de Novion, 2003, "A SANS investigation of the irradiationenhanced $\alpha-\alpha^{\prime}$ phases separation in 7-12 Cr martensitic steels," J. Nucl. Mater. 312, 236-248.

Mathon, M. H., M. Perrut, S. Y. Zhong, and Y. de Carlan, 2012, "Small angle neutron scattering study of martensitic/ferritic ODS alloys," J. Nucl. Mater. 428, 147-153.

Matricon, J., 1964, "Energy and elastic moduli of a lattice of vortex lines," Phys. Lett. 9, 289-291.

Maurer, Thomas, Sébastien Gautrot, Frédéric Ott, Grégory Chaboussant, Fatih Zighem, Laurent Cagnon, and Olivier Fruchart, 2014, "Ordered arrays of magnetic nanowires investigated by polarized small-angle neutron scattering," Phys. Rev. B 89, 184423.

May, R. P., 1994, "Geometrical optimization of neutron small-angle scattering instruments," J. Appl. Crystallogr. 27, 298-301.

Mayr, M., A. Moreo, J. A. Vergés, J. Arispe, A. Feiguin, and E. Dagotto, 2001, "Resistivity of mixed-phase manganites," Phys. Rev. Lett. 86, 135-138.

Mazumder, S., D. Sen, S. K. Roy, M. Hainbuchner, M. Baron, and H. Rauch, 2001, "Manifestation of the statistical nature of a medium in multiple small-angle scattering," J. Phys. Condens. Matter 13, 5089-5102.

Melnichenko, Y. B., and G. D. Wignall, 2007, "Small-angle neutron scattering in materials science: Recent practical applications," J. Appl. Phys. 102, 021101.

Mercone, S., V. Hardy, C. Martin, C. Simon, D. Saurel, and A. Brûlet, 2003, "Field dependence of the electronic phase separation in $\operatorname{Pr}_{0.67} \mathrm{Ca}_{0.33} \mathrm{MnO}_{3}$ by small-angle magnetic neutron scattering," Phys. Rev. B 68, 094422.
Mériguet, G., E. Dubois, M. Jardat, A. Bourdon, G. Demouchy, V. Dupuis, B. Farago, R. Perzynski, and P. Turq, 2006a, "Understanding the structure and the dynamics of magnetic fluids: coupling of experiment and simulation," J. Phys. Condens. Matter 18, S2685-S2696.

Mériguet, Guillaume, Fabrice Cousin, Emmanuelle Dubois, François Boué, Andrejs Cebers, Bela Farago, and Régine Perzynski, 2006b, "What Tunes the Structural Anisotropy of Magnetic Fluids under a Magnetic Field?" J. Phys. Chem. B 110, 4378-4386.

Messiah, A., 1990, Quantenmechanik, Bd. 2 (Walter de Gruyter, Berlin).

Metlov, K. L., and A. Michels, 2015, "Third-order effect in magnetic small-angle neutron scattering by a spatially inhomogeneous medium," Phys. Rev. B 91, 054404.

Metlov, K. L., and A. Michels, 2016, "Magnetic neutron scattering by magnetic vortices in thin submicron-sized soft ferromagnetic cylinders," Sci. Rep. 6, 25055.

Mettus, D., and A. Michels, 2015, "Small-angle neutron scattering correlation functions of bulk magnetic materials," J. Appl. Crystallogr. 48, 1437-1450.

Mettus, Denis, et al., 2017, "Magnetic small-angle neutron scattering on bulk metallic glasses: A feasibility study for imaging displacement fields," Phys. Rev. Mater. 1, 074403.

Meyer, A. J.P., and P. Taglang, 1956, "Propriétés magnétiques, antiferromagnétisme et ferromagnétisme de $\mathrm{MnAu}_{2}$," J. Phys. Radium 17, 457-465.

Meyer, E., H. J. Hug, and R. Bennewitz, 2004, Scanning Probe Microscopy: The Lab on a Tip (Springer, New York).

Meynell, S. A., M. N. Wilson, K. L. Krycka, B. J. Kirby, H. Fritzsche, and T. L. Monchesky, 2017, "Neutron study of in-plane skyrmions in MnSi thin films," Phys. Rev. B 96, 054402.

Mezei, F., 1986, "La nouvelle vague in polarized neutron scattering," Physica B+C (Amsterdam) 137B, 295.

Michels, A., 2010, "Correlation functions of the spin misalignment in magnetic small-angle neutron scattering," Phys. Rev. B 82, 024433.

Michels, A., 2014, "Magnetic small-angle neutron scattering of bulk ferromagnets," J. Phys. Condens. Matter 26, 383201.

Michels, A., F. Döbrich, M. Elmas, A. Ferdinand, J. Markmann, M. Sharp, H. Eckerlebe, J. Kohlbrecher, and R. Birringer, 2008, "Spin structure of nanocrystalline gadolinium," Europhys. Lett. 81, 66003.

Michels, A., M. Elmas, F. Döbrich, M. Ames, J. Markmann, M. Sharp, H. Eckerlebe, J. Kohlbrecher, and R. Birringer, 2009, "Porosity-induced spin disorder in nanocrystalline inertgascondensed iron," Europhys. Lett. 85, 47003.

Michels, A., S. Erokhin, D. Berkov, and N. Gorn, 2014, "Micromagnetic simulation of magnetic small-angle neutron scattering from two-phase nanocomposites," J. Magn. Magn. Mater. 350, $55-68$.

Michels, A., D. Honecker, S. Erokhin, and D. Berkov, 2015, "Comment on "Origin of Surface Canting within $\mathrm{Fe}_{3} \mathrm{O}_{4}$ Nanoparticles"," Phys. Rev. Lett. 114, 149701.

Michels, A., D. Mettus, D. Honecker, and K. L. Metlov, 2016, "Effect of Dzyaloshinski-Moriya interaction on elastic small-angle neutron scattering," Phys. Rev. B 94, 054424.

Michels, A., C. Vecchini, O. Moze, K. Suzuki, P. K. Pranzas, J. Kohlbrecher, and J. Weissmüller, 2006, "Dipolar correlations in a nanocomposite: A neutron scattering study of Nanoperm $\mathrm{Fe}_{89} \mathrm{Zr}_{7} \mathrm{~B}_{3} \mathrm{Cu}$," Phys. Rev. B 74, 134407.

Michels, A., R. N. Viswanath, J. G. Barker, R. Birringer, and J. Weissmüller, 2003, "Range of Magnetic Correlations in Nanocrystalline Soft Magnets,” Phys. Rev. Lett. 91, 267204. 
Michels, A., and J. Weissmüller, 2008, "Magnetic-field-dependent small-angle neutron scattering on random anisotropy ferromagnets," Rep. Prog. Phys. 71, 066501.

Michels, A., et al., 2011, "Influence of crystallite size and temperature on the antiferromagnetic helices of terbium and holmium metal," Phys. Rev. B 83, 224415.

Michels, A., et al., 2017, "Spin Structures of Textured and Isotropic Nd-Fe-B-Based Nanocomposites: Evidence for Correlated Crystallographic and Spin Textures," Phys. Rev. Applied 7, 024009.

Michels, A., et al., 2019, "Microstructural-defect-induced Dzyaloshinskii-Moriya interaction,” Phys. Rev. B 99, 014416.

Milde, P., et al., 2013, Science 340, 1076-1080.

Mildner, D. F. R., and R. Cubitt, 2012, "The effect of gravity on the Debye-Scherrer ring in small-angle neutron scattering," J. Appl. Crystallogr. 45, 124-126.

Miller, M. K., B. D. Wirth, and G. R. Odette, 2003, "Precipitation in neutron-irradiated $\mathrm{Fe}-\mathrm{Cu}$ and $\mathrm{Fe}-\mathrm{Cu}-\mathrm{Mn}$ model alloys: a comparison of APT and SANS data," Mater. Sci. Eng. A 353, 133-139.

Moon, R. M., T. Riste, and W.C. Koehler, 1969, "Polarization Analysis of Thermal-Neutron Scattering," Phys. Rev. 181, 920.

Morisaki-Ishii, R., et al., 2014, "Vortex lattice structure in $\mathrm{BaFe}_{2}\left(\mathrm{As}_{0.67} \mathrm{P}_{0.33}\right)_{2}$ by the small-angle neutron scattering technique," Phys. Rev. B 90, 125116.

Moriya, T., 1960, "Anisotropic superexchange interaction and weak ferromagnetism," Phys. Rev. 120, 91.

Mostovoy, Maxim, 2006, "Ferroelectricity in spiral magnets," Phys. Rev. Lett. 96, 067601.

Mühlbauer, S., B. Binz, F. Jonietz, C. Pfleiderer, A. Rosch, A. Neubauer, R. Georgii, and P. Böni, 2009, "Skyrmion lattice in a chiral Magnet," Science 323, 915.

Mühlbauer, S., G. Brandl, M. Månsson, and M. Garst, 2017, "Formation of incommensurate long-range magnetic order in the Dzyaloshinskii-Moriya antiferromagnet $\mathrm{Ba}_{2} \mathrm{CuGe}_{2} \mathrm{O}_{7}$ studied by neutron diffraction," Phys. Rev. B 96, 134409.

Mühlbauer, S., S. Gvasaliya, E. Ressouche, E. Pomjakushina, and A. Zheludev, 2012, "Phase diagram of the Dzyaloshinskii-Moriya helimagnet $\mathrm{Ba}_{2} \mathrm{CuGe}_{2} \mathrm{O}_{7}$ in canted magnetic fields," Phys. Rev. B 86, 024417.

Mühlbauer, S., A. Heinemann, A. Wilhelm, L. Karge, A. Ostermann, I. Defendi, A. Schreyer, W. Petry, and R. Gilles, 2016, "The new small-angle neutron scattering instrument SANS-1 at MLZcharacterization and first results," Nucl. Instrum. Methods Phys. Res., Sect. A 832, 297-305.

Mühlbauer, S., J. Kindervater, T. Adams, A. Bauer, U. Keiderling, and C. Pfleiderer, 2016, "Kinetic small angle neutron scattering of the skyrmion lattice in MnSi," New J. Phys. 18, 075017.

Mühlbauer, S., C. Pfleiderer, P. Böni, E. M. Forgan, E. H. Brandt, A. Wiedenmann, U. Keiderling, and G. Behr, 2011, "Time-resolved stroboscopic neutron scattering of vortex lattice dynamics in superconducting niobium," Phys. Rev. B 83, 184502.

Mühlbauer, S., C. Pfleiderer, P. Böni, M. Laver, E. M. Forgan, D. Fort, U. Keiderling, and G. Behr, 2009, "Morphology of the Superconducting Vortex Lattice in Ultrapure Niobium," Phys. Rev. Lett. 102, 136408.

Müller, Jan, Achim Rosch, and Markus Garst, 2016, "Edge instabilities and skyrmion creation in magnetic layers," New J. Phys. 18, 065006.

Münzer, W., et al., 2010, "Skyrmion lattice in the doped semiconductor $\mathrm{Fe}_{1-x} \mathrm{Co}_{x} \mathrm{Si}$," Phys. Rev. B 81, 041203.

Murakawa, H., Y. Onose, K. Ohgushi, S. Ishiwata, and Y. Tokura, 2008, "Generation of Electric Polarization with Rotating Magnetic Field in Helimagnet $\mathrm{ZnCr}_{2} \mathrm{Se}_{4}$," J. Phys. Soc. Jpn. 77, 043709.
Nagaev, E. L., 2001, "Colossal-magnetoresistance materials: manganites and conventional ferromagnetic semiconductors," Phys. Rep. 346, 387-531.

Nagaev, E. L., and A. I. Podel'shchikov, 1996, "Phase separation and resistivity jumps in Co compounds and other materials with lowspin-high-spin transitions," J. Phys. Condens. Matter 8, 5611.

Nagamiya, T., K. Nagata, and Y. Kitano, 1962, "Magnetization Process of a Screw Spin System," Prog. Theor. Phys. 27, 1253-1271.

Nagaosa, N., and Y. Tokura, 2013, "Topological properties and dynamics of magnetic skyrmions," Nat. Nanotechnol. 8, 899-911.

Nagornyi, A., V. Petrenko, M. Avdeev, L. Bulavin, and V. Aksenov, 2010, "Analysis of small-angle neutron scattering from very dilute magnetic fluids," J. Surf. Investig. X-ray, Synchrotron Neutron Tech. 4, 976-981.

Nakai, N., and K. Machida, 2015, "Interplay between effective mass anisotropy and Pauli paramagnetic effects in a multiband superconductor: Application to $\mathrm{Sr}_{2} \mathrm{RuO}_{4}$," Phys. Rev. B 92, 054505.

Nakajima, T., H. Oike, A. Kikkawa, E. P. Gilbert, N. Booth, K. Kakurai, Y. Taguchi, Y. Tokura, F. Kagawa, and T-h. Arima, 2017, "Skyrmion lattice structural transition in MnSi," Sci. Adv. 3, e1602562.

Nayak, A. K., V. Kumar, T. Ma, P. Werner, E. Pippel, R. Sahoo, F. Damay, U. K. Rössler, C. Felser, and S. S.P. Parkin, 2017, "Magnetic antiskyrmions above room temperature in tetragonal Heusler materials," Nature (London) 548, 561-566.

Nedelkoski, Z., et al., 2017, "Origin of reduced magnetization and domain formation in small magnetite nanoparticles," Sci. Rep. 7, 45997.

Neubauer, A., C. Pfleiderer, B. Binz, A. Rosch, R. Ritz, P. G. Niklowitz, and P. Böni, 2009, "Topological Hall Effect in the A Phase of MnSi,” Phys. Rev. Lett. 102, 186602.

Nii, Y., T. Nakajima, A. Kikkawa, Y. Yamasaki, K. Ohishi, J. Suzuki, Y. Taguchi, T. Arima, Y. Tokura, and Y. Iwasa, 2015, "Uniaxial stress control of skyrmion phase," Nat. Commun. 6, 8539.

Nisoli, C., R. Moessner, and P. Schiffer, 2013, "Colloquium: Artificial spin ice: Designing and imaging magnetic frustration," Rev. Mod. Phys. 85, 1473-1490.

Noguchi, S., S. Kawamata, K. Okuda, H. Nojiri, and M. Motokawa, 2002, "Evidence for the excited triplet of $\mathrm{Co}^{3+}$ in $\mathrm{LaCoO}_{3}$," Phys. Rev. B 66, 094404.

Odette, G. R., 1994, "Radiation-induced microstructural evolution in reactor pressure vessel steels," Mater. Res. Soc. Symp. Proc. 373, 137-148.

Odette, G. R., M. J. Alinger, and B. D. Wirth, 2008, "Recent developments in irradiation-resistant steels," Annu. Rev. Mater. Res. 38, 471-503.

Odette, G. R., and G. E. Lucas, 1998, "Recent progress in understanding reactor pressure vessel steel embrittlement," Radiat. Eff. Defects Solids 144, 189-231.

Ogrin, F. Y., S. L. Lee, M. Wismayer, T. Thomson, C. D. Dewhurst, R. Cubitt, and S. M. Weekes, 2006, "Micromagnetic simulation of small-angle neutron scattering from magnetic recording media," J. Appl. Phys. 99, 08G912.

Oike, H., A. Kikkawa, N. Kanazawa, Y. Taguchi, M. Kawasaki, Y. Tokura, and F. Kagawa, 2016, "Interplay between topological and thermodynamic stability in a metastable magnetic skyrmion lattice," Nat. Phys. 12, 62-66.

Okorokov, A. I., V. V. Runov, and A. G. Gukasov, 1978, "Threedimensional neutron polarimeter and spin dynamics investigation," Nucl. Instrum. Methods 157, 487.

Okorokov, A. I., V. V. Runov, B. P. Toperverg, A. D. Tretyakov, E. I. Maltsev, I. M. Puzei, and V.E. Mikhailova, 1986, "Study of spin waves in amorphous magnetic materials by polarized-neutron scattering," JETP Lett. 43, 503-507. 
Okubo, Tsuyoshi, Sungki Chung, and Hikaru Kawamura, 2012, "Multiple- $q$ States and the Skyrmion Lattice of the Triangular-Lattice Heisenberg Antiferromagnet under Magnetic Fields," Phys. Rev. Lett. 108, 017206.

Okuyama, D., K. Makino, M. Avdeev, K. Ohishi, K. Yamauchi, T. Oguchi, and K. Sato, 2017, "IHelical magnetic structure in cubic chiral crystal $\operatorname{Pr}_{r} \mathrm{Ru}_{3} \mathrm{Al}_{2}$," Abstract IUCR 2017.

Page, R. A., 1988, "Applications of small-angle scattering in ceramic research,” J. Appl. Crystallogr. 21, 795-804.

Pankhurst, Q. A., J. Connolly, S. K. Jones, and J. Dobson, 2003, "Applications of magnetic nanoparticles in biomedicine," J. Phys. D 36, R167.

Pappas, C., E. Lelièvre-Berna, P. Falus, P. M. Bentley, E. Moskvin, S. Grigoriev, P. Fouquet, and B. Farago, 2009, "Chiral Paramagnetic Skyrmion-like Phase in MnSi," Phys. Rev. Lett. 102, 197202.

Pareja, R., P. Parente, A. Muñoz, A. Radulescu, and V. de Castro, 2015, "Small-angle neutron scattering study of the nano-sized features in an oxide dispersion-strengthened Fe12Cr alloy," Philos. Mag. 95, 2450.

Park, Jongnam, Kwangjin An, Yosun Hwang, Je-Geun Park, Han-Jin Noh, Jae-Young Kim, Jae-Hoon Park, Nong-Moon Hwang, and Taeghwan Hyeon, 2004, "Ultra-large-scale syntheses of monodisperse nanocrystals," Nat. Mater. 3, 891-895.

Park, Jongnam, Jin Joo, Gu Kwon Soon, Youngjin Jang, and Taeghwan Hyeon, 2007, "Synthesis of monodisperse spherical nanocrystals," Angew. Chem., Int. Ed. Engl. 46, 4630-4660.

Park, Jongnam, et al., 2005, "One-Nanometer-Scale Size-Controlled Synthesis of Monodisperse Magnetic Iron Oxide Nanoparticles," Angew. Chem., Int. Ed. Engl. 117, 2932-2937.

Pasupathy, A. N., A. Pushp, K. K. Gomes, C. V. Parker, J. Wen, Z. $\mathrm{Xu}, \mathrm{G}$. Gu, S. Ono, Y. Ando, and A. Yazdani, 2008, "Electronic origin of the inhomogeneous pairing interaction in the high- $T_{C}$ superconductor $\mathrm{Bi}_{2} \mathrm{Sr}_{2} \mathrm{CaCu}_{2} \mathrm{O}_{8+\delta}$," Science 320, 196 .

Paul, D. McK., C. V. Tomy, C. M. Aegerter, R. Cubitt, S. H. Lloyd, E. M. Forgan, S. L. Lee, and M. Yethiraj, 1998, "Nonlocal effects and vortex lattice transitions in $\mathrm{YNi}_{2} \mathrm{~B}_{2} \mathrm{C}$," Phys. Rev. Lett. 80, $1517-1520$.

Pautrat, A., and A. Brûlet, 2014, "Temperature dependence of clusters with attracting vortices in superconducting niobium studied by neutron scattering," J. Phys. Condens. Matter 26, 232201.

Pautrat, A., A. Brûlet, C. Simon, and P. Mathieu, 2012, "Flux-lines lattice order and critical current studied by time-of-flight smallangle neutron scattering," Phys. Rev. B 85, 184504.

Pautrat, A., J. Scola, C. Simon, A. Brûlet, C. Goupil, M. J. Higgins, and S. Bhattacharya, 2005, "Metastable states of a flux-line lattice studied by transport and small-angle neutron scattering," Phys. Rev. B 71, 064517.

Pautrat, A., C. Simon, A. Brûlet, C. Dewhurst, S. Bhattacharya, and M. J. Higgins, 2009, "Structure of the flux lines lattice in $\mathrm{NbSe}_{2}$ : Equilibrium state and influence of the magnetic history," Phys. Rev. B 79, 184511.

Pautrat, A., C. Simon, C. Goupil, A. Brûlet, C. Dewhurst, and A. Rykov, 2007, "Persistence of an ordered flux line lattice above the second peak in $\mathrm{Bi}_{2} \mathrm{Sr}_{2} \mathrm{CaCu}_{2} \mathrm{O}_{8+\delta}$," Phys. Rev. B 75, 224512.

Pauw, B.R., 2013, "Everything SAXS: small-angle scattering pattern collection and correction," J. Phys. Condens. Matter 25, 383201 .

Pedersen, J. S., 1997, "Analysis of small-angle scattering data from colloids and polymer solutions: modeling and least-squares fitting," Adv. Colloid Interface Sci. 70, 171-210.

Pedersen, J. S., D. Posselt, and K. Mortensen, 1990, "Analytical treatment of the resolution function for small-angle scattering," J. Appl. Crystallogr. 23, 321.
Périgo, E. A., E. P. Gilbert, K. L. Metlov, and A. Michels, 2014, "Experimental observation of magnetic poles inside bulk magnets via $\mathbf{q} \neq 0$ fourier modes of magnetostatic field," New J. Phys. 16, 123031.

Périgo, E. A., E. P. Gilbert, and A. Michels, 2015, "Magnetic SANS study of a sintered Nd-Fe-B magnet: Estimation of defect size," Acta Mater. 87, 142.

Périgo, E. A., I. Titov, R. Weber, D. Honecker, E. P. Gilbert, M. F. De Campos, and A. Michels, 2016, "Small-angle neutron scattering study of coercivity enhancement in grain-boundary-diffused Nd-Fe-B sintered magnets," J. Alloys Compd. 677, 139-142.

Perrut, M., M.H. Mathon, and D. Delagnes, 2012, "Small-angle neutron scattering of multiphase secondary hardening steels," J. Mater. Sci. 47, 1920.

Petoukhov, A. K., et al., 2006, "Compact magnetostatic cavity for polarised ${ }^{3} \mathrm{He}$ neutron spin filter cells," Nucl. Instrum. Methods Phys. Res., Sect. A 560, 480.

Petrenko, V. I., M. V. Avdeev, L. A. Bulavin, L. Almasy, N. A. Grigoryeva, and V. L. Aksenov, 2016, "Effect of surfactant excess on the stability of low-polarity ferrofluids probed by small-angle neutron scattering," Crystallogr. Rep. 61, 121-125.

Pfleiderer, C., S. R. Julian, and G. G. Lonzarich, 2001, "Non-fermi liquid nature of the normal state of itinerant-electron ferromagnets," Nature (London) 414, 427.

Pfleiderer, C., G. J. McMullan, and G. G. Lonzarich, 1995, "Pressure induced crossover of the magnetic transition from second to first order near the quantum critical point in MnSi," Physica B (Amsterdam) 206-207, 847-849.

Pfleiderer, C., D. Reznik, L. Pintschovius, and J. Haug, 2007, "Magnetic Field and Pressure Dependence of Small Angle Neutron Scattering in MnSi," Phys. Rev. Lett. 99, 156406.

Pfleiderer, C., D. Reznik, L. Pintschovius, H. v. Löhneysen, M. Garst, and A. Rosch, 2004, "Partial order in the non-Fermi-liquid phase of MnSi," Nature (London) 427, 227-231.

Phelan, D., Kanwal Preet Bhatti, M. Taylor, Shun Wang, and C. Leighton, 2014, "Magnetically inhomogeneous ground state below the first-order valence transition in $\left(\mathrm{Pr}_{1-y} \mathrm{Y}_{y}\right)_{0.7} \mathrm{Ca}_{0.3} \mathrm{CoO}_{3-\delta}$," Phys. Rev. B 89, 184427.

Phelan, D., Despina Louca, K. Kamazawa, S.-H. Lee, S. N. Ancona, S. Rosenkranz, Y. Motome, M. F. Hundley, J. F. Mitchell, and Y. Moritomo, 2006, "Spin incommensurability and two phase competition in cobaltites," Phys. Rev. Lett. 97, 235501.

Phelan, D., Y. Suzuki, S. Wang, A. Huq, and C. Leighton, 2013, "Structural, transport, and magnetic properties of narrow bandwidth $\mathrm{Nd}_{1-x} \mathrm{Ca}_{x} \mathrm{CoO}_{3-\delta}$ and comparisons to $\operatorname{Pr}_{1-x} \mathrm{Ca}_{x} \mathrm{CoO}_{3-\delta}$, Phys. Rev. B 88, 075119.

Phelan, D., et al., 2006, "Nanomagnetic droplets and implications to orbital ordering in $\mathrm{La}_{1-x} \mathrm{Sr}_{x} \mathrm{CoO}_{3}$," Phys. Rev. Lett. 96, 027201.

Plumier, R., 1966, "Étude par diffraction de neutrons de l'antiferromagnétisme hélicoïdal du spinelle $\mathrm{ZnCr}_{2} \mathrm{Se}_{4}$ en présence d'un champ magnétique," J. Phys. France 27, 213-219.

Podlesnyak, A., G. Ehlers, M. Frontzek, A. S. Sefat, A. Furrer, Th. Strässle, E. Pomjakushina, K. Conder, F. Demmel, and D. I. Khomskii, 2011, "Effect of carrier doping on the formation and collapse of magnetic polarons in lightly hole-doped $\mathrm{La}_{1-x} \mathrm{Sr}_{x} \mathrm{CoO}_{3}$," Phys. Rev. B 83, 134430.

Podlesnyak, A., S. Streule, J. Mesot, M. Medarde, E. Pomjakushina, K. Conder, A. Tanaka, M. W. Haverkort, and D. I. Khomskii, 2006, "Spin-state transition in $\mathrm{LaCoO}_{3}$ : Direct neutron spectroscopic evidence of excited magnetic states," Phys. Rev. Lett. 97, 247208. Podlesnyak, A., et al., 2008, "Spin-state polarons in lightly-holedoped $\mathrm{LaCoO}_{3}$," Phys. Rev. Lett. 101, 247603. 
Pop, L. M., J. Hilljegerdes, S. Odenbach, and A. Wiedenmann, 2004, "The microstructure of ferrofluids and their rheological properties," Appl. Organomet. Chem. 18, 523-528.

Pop, Loredana Mirela, and Stefan Odenbach, 2006, "Investigation of the microscopic reason for the magnetoviscous effect in ferrofluids studied by small angle neutron scattering," J. Phys. Condens. Matter 18, S2785.

Porod, G., 1982a, in Small Angle X-ray Scattering, edited by O. Glatter and O. Kratky (Academic Press, New York), pp. 17-51.

Porod, G., 1982b, in Small Angle X-ray Scattering, edited by O. Glatter and O. Kratky (Academic Press, New York), pp. 247-252.

Prozorov, R., and R. W. Giannetta, 2006, "Magnetic penetration depth in unconventional superconductors," Supercond. Sci. Technol. 19, R41-R67.

Qin, Y., T.A. Tyson, K. Pranzas, and H. Eckerlebe, 2008, "Observation of ferromagnetic clusters in $\mathrm{Bi}_{0.125} \mathrm{Ca}_{0.875} \mathrm{MnO}_{3}$," J. Phys. Condens. Matter 20, 195209.

Raccah, P. M., and J. B. Goodenough, 1967, "First-Order LocalizedElectron $\leftrightarrows$ Collective-Electron Transition in $\mathrm{LaCoO}_{3}$," Phys. Rev. 155, 932-943.

Radaelli, P. G., R. M. Ibberson, D. N. Argyriou, H. Casalta, K. H. Andersen, S.-W. Cheong, and J. F. Mitchell, 2001, "Mesoscopic and microscopic phase segregation in manganese perovskites," Phys. Rev. B 63, 172419.

Radlinski, A. P., M. Mastalerz, A. L. Hinde, M. Hainbuchner, H. Rauch, M. Baron, J. S. Lin, L. Fan, and P. Thiyagarajan, 2004, "Application of SAXS and SANS in evaluation of porosity, pore size distribution and surface area of coal," Int. J. Coal Geology 59, 245-271.

Raghuwanshi, V. S., R. Harizanova, S. Haas, D. Tatchev, I. Gugov, C. Dewhurst, C. Rüssel, and A. Hoell, 2014, "Magnetic nanocrystals embedded in silicate glasses studied by polarized SANS,' J. NonCryst. Solids 385, 24-29.

Raghuwanshi, Vikram Singh, Ruzha Harizanova, Dragomir Tatchev, Armin Hoell, and Christian Rüssel, 2015, "Structural analysis of Fe-Mn-O nanoparticles in glass ceramics by small angle scattering," J. Solid State Chem. 222, 103-110.

Rajnak, M., et al., 2017, "Structure and viscosity of a transformer oilbased ferrofluid under an external electric field," J. Magn. Magn. Mater. 431, 99-102.

Rajnak, Michal, Viktor I. Petrenko, Mikhail V. Avdeev, Olexandr I. Ivankov, Artem Feoktystov, Bystrik Dolnik, Juraj Kurimsky, Peter Kopcansky, and Milan Timko, 2015, "Direct observation of electric field induced pattern formation and particle aggregation in ferrofluids," Appl. Phys. Lett. 107, 073108.

Ramazanoglu, M., M. Laver, W. Ratcliff, S. M. Watson, W. C. Chen, A. Jackson, K. Kothapalli, Seongsu Lee, S.-W. Cheong, and V. Kiryukhin, 2011, "Local weak ferromagnetism in single-crystalline ferroelectric $\mathrm{BiFeO}_{3}$," Phys. Rev. Lett. 107, 207206.

Rastovski, C., C. D. Dewhurst, W. J. Gannon, D. C. Peets, H. Takatsu, Y. Maeno, M. Ichioka, K. Machida, and M. R. Eskildsen, 2013, "Anisotropy of the Superconducting State in $\mathrm{Sr}_{2} \mathrm{RuO}_{4}$," Phys. Rev. Lett. 111, 087003.

Rastovski, C., K. J. Schlesinger, W. J. Gannon, C. D. Dewhurst, L. DeBeer-Schmitt, N. D. Zhigadlo, J. Karpinski, and M. R. Eskildsen, 2013, "Persistence of Metastable Vortex Lattice Domains in $\mathrm{MgB}_{2}$ in the Presence of Vortex Motion," Phys. Rev. Lett. 111, 107002.

Reimann, T., A. Bauer, C. Pfleiderer, P. Böni, P. Trtik, A. Tremsin, M. Schulz, and S. Mühlbauer, 2018, "Neutron diffractive imaging of the skyrmion lattice nucleation in MnSi," Phys. Rev. B 97, 020406.

Reimann, T., S. Mühlbauer, M. Schulz, B. Betz, A. Kaestner, V. Pipich, P. Böni, and C. Grünzweig, 2015, "Visualizing the morphology of vortex lattice domains in a bulk type-II superconductor," Nat. Commun. 6, 8813.

Rennie, A. R., et al., 2013, "Learning about sans instruments and data reduction from round robin measurements on samples of polystyrene latex," J. Appl. Crystallogr. 46, 1289-1297.

Rhyne, J. J., and G. E. Fish, 1985, "Small-angle neutron scattering evidence for the absence of long-range magnetic order in amorphous $\mathrm{Fe}_{91} \mathrm{Zr}_{9}$," J. Appl. Phys. 57, 3407-3409.

Ridier, Karl, Béatrice Gillon, Grégory Chaboussant, Laure Catala, Sandra Mazérat, Eric Rivière, and Talal Mallah, 2017, "Individual-collective crossover driven by particle size in dense assemblies of superparamagnetic nanoparticles," Eur. Phys. J. B 90, 77.

Riseman, T. M., et al., 1998, "Observation of a square flux-line lattice in the unconventional superconductor $\mathrm{Sr}_{2} \mathrm{RuO}_{4}$," Nature (London) 396, 242-245.

Ritter, C., M. R. Ibarra, J. M. De Teresa, P. A. Algarabel, C. Marquina, J. Blasco, J. García, S. Oseroff, and S-W. Cheong, 1997, "Influence of oxygen content on the structural, magnetotransport, and magnetic properties of $\mathrm{LaMnO}_{3+\delta}$," Phys. Rev. B 56, 8902-8911.

Ritz, R., M. Halder, M. Wagner, C. Franz, A. Bauer, and C. Pfleiderer, 2013, "Formation of a topological non-Fermi liquid in MnSi," Nature (London) 497, 231-234.

Roessli, B., J. Schefer, G. A. Petrakovskii, B. Ouladdiaf, M. Boehm, U. Staub, A. Vorotinov, and L. Bezmaternikh, 2001, "Formation of a Magnetic Soliton Lattice in Copper Metaborate," Phys. Rev. Lett. 86, 1885-1888.

Romming, N., C. Hanneken, M. Menzel, J. E. Bickel, B. Wolter, K. von Bergmann, A. Kubetzka, and R. Wiesendanger, 2013, "Writing and deleting single magnetic skyrmions," Science 341, 636-639.

Ronning, F., et al., 2017, "Electronic in-plane symmetry breaking at field-tuned quantum criticality in $\mathrm{CeRhIn}_{5}$," Nature (London) 548, 313-317.

Ropka, Z., and R. J. Radwanski, 2003, “5D term origin of the excited triplet in $\mathrm{LaCoO}_{3}$," Phys. Rev. B 67, 172401.

Rößler, U. K., A. N. Bogdanov, and C. Pfleiderer, 2006, "Spontaneous skyrmion ground states in magnetic metals," Nature (London) 442, 797-801.

Rothwell, W. S., 1968, "Small-Angle X-Ray Scattering from Glassy Carbon,” J. Appl. Phys. 39, 1840-1845.

Ruderman, M. A., and C. Kittel, 1954, "Indirect Exchange Coupling of Nuclear Magnetic Moments by Conduction Electrons," Phys. Rev. 96, 99.

Rybakov, Filipp. N., Aleksandr B. Borisov, Stefan Blügel, and Nikolai S. Kiselev, 2015, Phys. Rev. Lett. 115, 117201.

Sachan, M., C. Bonnoit, S. A. Majetich, Y. Ijiri, P. O. Mensah-Bonsu, J. A. Borchers, and J. J. Rhyne, 2008, "Field evolution of magnetic correlation lengths in $\epsilon$-Co nanoparticle assemblies," Appl. Phys. Lett. 92, 152503.

Saito, K., T. Ueno, M. Yano, M. Harada, T. Shoji, N. Sakuma, A. Manabe, A. Kato, U. Keiderling, and K. Ono, 2015, "Magnetization reversal of a $\mathrm{Nd}-\mathrm{Cu}$-infiltrated $\mathrm{Nd}-\mathrm{Fe}-\mathrm{B}$ nanocrystalline magnet observed with small-angle neutron scattering," J. Appl. Phys. 117, 17B302.

Salamon, M. B., P. Lin, and S. H. Chun, 2002, "Colossal Magnetoresistance is a Griffiths Singularity,” Phys. Rev. Lett. 88, 197203.

Sandratskii, L. M., 1998, "Noncollinear magnetism in itinerantelectron systems: Theory and applications," Adv. Phys. 47, 91-160.

Sandratskii, L. M., and J. Kübler, 1996, "Noncollinear magnetism: effects of symmetry and relativity," Mod. Phys. Lett. B 10, 189-201.

Sankar, Sandra, A. E. Berkowitz, D. Dender, J. A. Borchers, R. W. Erwin, S. R. Kline, and David J. Smith, 2000, "Magnetic corre- 
lations in non-percolated $\mathrm{Co}-\mathrm{SiO}_{2}$ granular films," J. Magn. Magn. Mater. 221, 1-9.

Saranu, S., A. Grob, J. Weissmüller, and U. Herr, 2008, "Micromagnetic structures of nanocrystalline ferromagnetic materials comparison of simulation and experiment," Phys. Status Solidi A 205, 1774-1778.

Saroun, J., 2007, "Evaluation of multiple small-angle neutron scattering including magnetic interactions," J. Appl. Crystallogr. 40, s701.

Saurel, D., A. Brûlet, A. Heinemann, C. Martin, S. Mercone, and Ch. Simon, 2006, "Magnetic field dependence of the magnetic phase separation in $\operatorname{Pr}_{1-x} \mathrm{Ca}_{x} \mathrm{MnO}_{3}$ manganites studied by small-angle neutron scattering," Phys. Rev. B 73, 094438.

Saurel, D., Ch. Simon, A. Brûlet, A. Heinemann, and C. Martin, 2007, "Small-angle neutron scattering study of the steplike magnetic transformation in $\operatorname{Pr}_{0.70} \mathrm{Ca}_{0.30} \mathrm{MnO}_{3}$," Phys. Rev. B 75, 184442.

Saurel, D., Ch. Simon, A. Pautrat, C. Martin, C. Dewhurst, and A. Brûlet, 2010, "Evolution of the conducting phase topology at the percolation threshold in colossal magnetoresistance manganites: A magnetic small-angle neutron scattering study," Phys. Rev. B 82, 054427.

Schärpf, O., and H. Capellmann, 1993, "The XYZ-Difference Method with Polarized Neutrons and the Separation of Coherent, Spin Incoherent, and Magnetic Scattering Cross Sections in a Multidetector," Phys. Status Solidi A 135, 359.

Schelten, J., and R. W. Hendricks, 1978, "Recent developments in $\mathrm{X}$-ray and neutron small-angle scattering instrumentation and data analysis," J. Appl. Crystallogr. 11, 297-324.

Schelten, J., G. Lippmann, and H. Ullmaier, 1974, "Neutron Diffraction Studies of the Morphology of Flux Line Crystals in Type II Superconductors," J. Low Temp. Phys. 14, 213-226.

Schelten, J., and W. Schmatz, 1980, "Multiple-scattering treatment for small-angle scattering problems," J. Appl. Crystallogr. 13, 385-390.

Schelten, J., H. Ullmaier, and W. Schmatz, 1971, "Neutron Diffraction by Vortex Lattices in Superconducting $\mathrm{Nb}$ and $\mathrm{Nb}_{0.73} \mathrm{Ta}_{0.27}$," Phys. Status Solidi B 48, 619-628.

Schlömann, E., 1967, "Properties of Magnetic Materials with a Nonuniform Saturation Magnetization. I. General Theory and Calculation of the Static Magnetization," J. Appl. Phys. 38, 5027.

Schmatz, W., T. Springer, J. Schelten, and K. Ibel, 1974, "Neutron Small-Angle Scattering: Experimental Techniques and Applications," J. Appl. Crystallogr. 7, 96-116.

Schmidt, P. W., 1991, "Small-angle scattering studies of disordered, porous and fractal systems," J. Appl. Crystallogr. 24, 414-435.

Schober, H., 2014, "An introduction to the theory of nuclear neutron scattering in condensed matter," J. Neutron Res. 17, 109-357.

Schober, M., E. Eidenberger, H. Leitner, P. Staron, D. Reith, and R. Podloucky, 2010, "A critical consideration of magnetism and composition of (bcc) $\mathrm{Cu}$ precipitates in (bcc) Fe," Appl. Phys. A 99, 697-704.

Schofield, A. J., 1999, "Non-Fermi liquids," Contemp. Phys. 40, 95-112.

Schrefl, T., H. Kronmüller, and J. Fidler, 1993, "Exchange hardening in nano-structured two-phase permanent magnets," J. Magn. Magn. Mater. 127, L273.

Schweizer, J., 2006, in Neutron Scattering from Magnetic Materials, edited by T. Chatterji (Elsevier, New York), pp. 153-213.

Segawa, K., and Y. Ando, 2009, "Magnetic and Transport Properties of FeAs Single Crystals,” J. Phys. Soc. Jpn. 78, 104720.
Seki, S., S. Ishiwata, and Y. Tokura, 2012, "Magnetoelectric nature of skyrmions in a chiral magnetic insulator $\mathrm{Cu}_{2} \mathrm{OSeO}_{3}$," Phys. Rev. B 86, 060403.

Seki, S., J.-H. Kim, D. S. Inosov, R. Georgii, B. Keimer, S. Ishiwata, and Y. Tokura, 2012, "Formation and rotation of skyrmion crystal in the chiral-lattice insulator $\mathrm{Cu}_{2} \mathrm{OSeO}_{3}$," Phys. Rev. B 85, 220406.

Seki, S., X. Z. Yu, S. Ishiwata, and Y. Tokura, 2012, "Observation of Skyrmions in a Multiferroic Material," Science 336, 198-201.

Sellmyer, D., and R. Skomski, 2006, Eds., Advanced Magnetic Nanostructures (Springer, New York).

Selte, K., A. Kjekshus, and A. F. Andresen, 1972, "Magnetic Structure and Properties of FeAs," Acta Chem. Scand. 26, 3101-3113.

Señarís-Rodríguez, M. A., and J. B. Goodenough, 1995, "Magnetic and transport properties of the system $\mathrm{La}_{1-x} \mathrm{Sr}_{x} \mathrm{CoO}_{3-\delta}(0<$ $x \leq 0.50)$, , J. Solid State Chem. 118, 323-336.

Sepehri-Amin, H., Lihua Liu, T. Ohkubo, M. Yano, T. Shoji, A. Kato, T. Schrefl, and K. Hono, 2015, "Microstructure and temperature dependent of coercivity of hot-deformed Nd-Fe-B magnets diffusion processed with Pr-Cu alloy," Acta Mater. 99, 297-306.

Sepehri-Amin, H., T. Ohkubo, S. Nagashima, M. Yano, T. Shoji, A. Kato, T. Schrefl, and K. Hono, 2013, "High-coercivity ultrafinegrained anisotropic Nd-Fe-B magnets processed by hot deformation and the $\mathrm{Nd}-\mathrm{Cu}$ grain boundary diffusion process," Acta Mater. 61, 6622-6634.

Sepehri-Amin, H., T. Ohkubo, T. Nishiuchi, S. Hirosawa, and K. Hono, 2010, "Coercivity enhancement of hydrogenationdisproportionation-desorption-recombination processed $\mathrm{Nd}-\mathrm{Ne}-\mathrm{N}$ powders by the diffusion of Nd-Cu eutectic alloys," Scr. Mater. 63, 1124-1127.

Sergienko, I. A., and E. Dagotto, 2006, "Role of the DzyaloshinskiiMoriya interaction in multiferroic perovskites," Phys. Rev. B 73, 094434

Shapiro, S. M., G. Shirane, B. H. Verbeek, G. J. Nieuwenhuys, and J. A. Mydosh, 1980, "Neutron scattering studies of the reentrant spinglass system PdFeMn," Solid State Commun. 36, 167-170.

Shibata, T., B. Bunker, J.F. Mitchell, and P. Schiffer, 2002, "Indications of intrinsic chemical and structural inhomogeneity in lightly doped $\mathrm{La}_{1-x} \mathrm{Sr}_{x} \mathrm{MnO}_{3}$," Phys. Rev. Lett. 88, 207205.

Shirane, G., J. M. Tranquada, and S. M. Shapiro, 2002, Neutron Scattering with a Triple-Axis SpectrometerBasic Techniques (Cambridge University Press, Cambridge, England).

Shu, S., B. D. Wirth, P. B. Wells, D. D. Morgan, and G. R. Odette, 2018, "Multi-technique characterization of the precipitates in thermally aged and neutron irradiated $\mathrm{Fe}-\mathrm{Cu}$ and $\mathrm{Fe}-\mathrm{Cu}-\mathrm{Mn}$ model alloys: atom probe tomography reconstruction implications," Acta Mater. 146, 237-252.

Shull, C. G., E. O. Wollan, and W. C. Koehler, 1951, "Neutron Scattering and Polarization by Ferromagnetic Materials," Phys. Rev. 84, 912.

Siegfried, S.-A., E. V. Altynbaev, N. M. Chubova, V. Dyadkin, D. Chernyshov, E. V. Moskvin, D. Menzel, A. Heinemann, A. Schreyer, and S. V. Grigoriev, 2015, "Controlling the dzyaloshinskii-moriya interaction to alter the chiral link between structure and magnetism for $\mathrm{Fe}_{1-x} \mathrm{Co}_{x} \mathrm{Si}$," Phys. Rev. B 91, 184406.

Siegfried, S.-A., A. S. Sukhanov, E. V. Altynbaev, D. Honecker, A. Heinemann, A. V. Tsvyashchenko, and S. V. Grigoriev, 2017, "Spin-wave dynamics in the helimagnet FeGe studied by smallangle neutron scattering," Phys. Rev. B 95, 134415.

Simon, Ch., S. Mercone, N. Guiblin, C. Martin, A. Brûlet, and G. André, 2002, "Microphase separation in $\operatorname{Pr}_{0.67} \mathrm{Ca}_{0.33} \mathrm{MnO}_{3}$ by small-angle neutron scattering," Phys. Rev. Lett. 89, 207202. 
Siratori, K., J. Akimitsu, E. Kita, and M. Nishi, 1980, "A Method of Controlling the Sense of the Screw Spin Structure," J. Phys. Soc. Jpn. 48, 1111-1114.

Skomski, R., 2003, "Nanomagnetics,” J. Phys. Condens. Matter 15, R841.

Skyrme, T. H., 1961a, "A non-linear field theory," Proc. R. Soc. A 260, 127.

Skyrme, T. H., 1961b, "Particle states of a quantized meson field," Proc. R. Soc. A 262, 237.

Skyrme, T.H., 1962, "A unifield field theory of mesons and baryons," Nucl. Phys. 31, 556.

Smith, R. X., M. J. R. Hoch, P. L. Kuhns, W. G. Moulton, A. P. Reyes, G. S. Boebinger, J. Mitchell, and C. Leighton, 2008, "Spin polarons in $\mathrm{La}_{1-x} \mathrm{Sr}_{x} \mathrm{CoO}_{3}$ single crystals," Phys. Rev. B 78, 092201.

Sondhi, S. L., A. Karlhede, S. A. Kivelson, and E. H. Rezayi, 1993, "Skyrmions and the crossover from the integer to fractional quantum Hall effect at small Zeeman energies," Phys. Rev. B 47, 16419-16426.

Sprouster, D. J., J. Sinsheimer, E. Dooryhee, S. K. Ghose, P. Wells, T. Stan, N. Almirall, G. R. Odette, and L. E. Ecker, 2016, "Structural characterization of nanoscale intermetallic precipitates in highly neutron irradiated reactor pressure vessel steels," Scr. Mater. 113, $18-22$.

Squires, G. L., 1978, Introduction to the Theory of Thermal Neutron Scattering (Dover Publications, New York).

Steele, L. E., 1975, Neutron Irradiation Embrittlement of Reactor Pressure Vessel Steels (International Atomic Energy Agencya, Vienna).

Stuhrmann, H. B., 2004, "Unique aspects of neutron scattering for the study of biological systems," Rep. Prog. Phys. 67, 1073-1115.

Suzuki, K., and G. Herzer, 2006, in Advanced Magnetic Nanostructures, edited by D. Sellmyer and R. Skomski (Springer, New York), pp. 365-401.

Svergun, D. I., and M.H. J. Koch, 2003, "Small-angle scattering studies of biological macromolecules in solution," Rep. Prog. Phys. 66, 1735-1782.

Svergun, D. I., M. H. J. Koch, P. A. Timmins, and R. P. May, 2013, Small Angle X-Ray and Neutron Scattering from Solutions of Biological Macromolecules (Oxford Science Publications, New York).

Swift, J., 1976, "Fluctuations near the nematic-smectic-C phase transition," Phys. Rev. A 14, 2274.

Szary, P., D. Kaiser, I.-P. Bick, D. Lott, A. Heinemann, C. Dewhurst, R. Birringer, and A. Michels, 2016, "Magnetic field-dependent spin structures of nanocrystalline holmium," J. Appl. Crystallogr. 49, 533-538.

Takanaka, K., 1971, "Flux-Line Lattice of Pure Type II Superconductors with Anisotropic Fermi Surface," Prog. Theor. Phys. 46, 1301-1306.

Takanaka, K., 1973a, "Correlation between Flux-Line Lattice and Crystal Axis in a Mixed State of Superconductors," Prog. Theor. Phys. 50, 365-369.

Takanaka, K., 1973b, "Flux-Line Lattices of Pure Type II Superconductor with Anisotropic Fermi Surface $(T \sim 0)$," Prog. Theor. Phys. 49, 64-75.

Takeda, M., J. Suzuki, T. Akiya, and H. Kato, 2012, "Small-Angle Neutron Scattering Measurements of the Averaged Internal Structures in Neodymium-Iron-Boron (Nd-Fe-B) Sintered Magnets," J. Jpn. Inst. Met. 76, 165-176.

Tasset, F., 1989, "Zero field neutron polarimetry," Physica B (Amsterdam) 156-157, 627.
Tatchev, Dragomir, Andre Heinemann, Albrecht Wiedenmann, and Armin Hoell, 2004, "Determination of particle size distributions by small-angle scattering with polarized neutrons using maximumentropy method," J. Appl. Crystallogr. 37, 40-47.

Thiemann, S. L., Z. Radovic, and V. G. Kogan, 1989, "Field structure of vortex lattices in uniaxial superconductors," Phys. Rev. B 39, 11406.

Thiyagarajan, P., 2003, "Characterization of materials of industrial importance using small-angle scattering techniques," J. Appl. Crystallogr. 36, 373-380.

Tinkham, M., 1996, Introduction to Superconductivity (McGrawHill, New York), 2nd ed.

Togawa, Y., T. Koyama, K. Takayanagi, S. Mori, Y. Kousaka, J. Akimitsu, S. Nishihara, K. Inoue, A.S. Ovchinnikov, and J. Kishine, 2012, "Chiral Magnetic Soliton Lattice on a Chiral Helimagnet,” Phys. Rev. Lett. 108, 107202.

Tokunaga, Y., X. Z. Yu, J. S. White, H. M. Rønnow, D. Morikawa, Y. Taguchi, and Y. Tokura, 2015, "A new class of chiral materials hosting magnetic skyrmions beyond room temperature," Nat. Commun. 6, 7638.

Tokura, Y., H. Kuwahara, Y. Moritomo, Y. Tomioka, and A. Asamitsu, 1996, "Competing instabilities and metastable states in (Nd, Sm) $)_{1 / 2} \mathrm{Sr}_{1 / 2} \mathrm{MnO}_{3}$," Phys. Rev. Lett. 76, 3184-3187.

Tokura, Y., and S. Seki, 2010, "Multiferroics with Spiral Spin Orders," Adv. Mater. 22, 1554-1565.

Tokura, Y., and Y. Tomioka, 1999, "Colossal magnetoresistive manganites," J. Magn. Magn. Mater. 200, 1-23.

Tomioka, Y., and Y. Tokura, 2004, "Global phase diagram of perovskite manganites in the plane of quenched disorder versus one-electron bandwidth," Phys. Rev. B 70, 014432.

Toperverg, B. P., V. V. Deriglazov, and V. E. Mikhailova, 1993, “On the studies of spin-wave dynamics in ferromagnets by polarized neutron scattering," Physica B (Amsterdam) 183, 326-330.

Torija, M. A., M. Sharma, J. Gazquez, M. Varela, Ch. He, J. Schmitt, J. A. Borchers, M. Laver, S. El-Khatib, and C. Leighton, 2011, "Chemically driven nanoscopic magnetic phase separation at the $\mathrm{SrTiO}_{3}(001) / \mathrm{La}_{1-x} \mathrm{Sr}_{x} \mathrm{CoO}_{3}$ interface," Adv. Mater. 23, 27112715.

Tsubouchi, S., T. Kyômen, M. Itoh, P. Ganguly, M. Oguni, Y. Shimojo, Y. Morii, and Y. Ishii, 2002, "Simultaneous metalinsulator and spin-state transitions in $\mathrm{Pr}_{0.5} \mathrm{Ca}_{0.5} \mathrm{CoO}_{3}$," Phys. Rev. B 66, 052418.

Uehara, M., S. Mori, C.H. Chen, and S.-W. Cheong, 1999, "Percolative phase separation underlies colossal magnetoresistance in mixed-valent manganites," Nature (London) 399, 560.

Ueland, B. G., J. W. Lynn, M. Laver, Y. J. Choi, and S.-W. Cheong, 2010, "Origin of electric-field-induced magnetization in multiferroic $\mathrm{HoMnO}_{3}$," Phys. Rev. Lett. 104, 147204.

Ujihara, T., and K. Osamura, 2000, "Kinetic analysis of spinodal decomposition process in $\mathrm{Fe}-\mathrm{Cr}$ alloys by small angle neutron scattering," Acta Mater. 48, 1629-1637.

Ukai, S., and M. Fujiwara, 2002, "Perspective of ODS alloys application in nuclear environments," J. Nucl. Mater. 307-311, 749-757.

Ulbricht, A., 2006, "Untersuchungen an neutronenbestrahlten Reaktordruckbehälterstählen mit Neutronen-Kleinwinkelstreuung," Ph.D. thesis (TU Bergakademie Freiberg), unpublished.

Vasiliu-Doloc, L., S. Rosenkranz, R. Osborn, S. K. Sinha, J. W. Lynn, J. Mesot, O. H. Seeck, G. Preosti, A. J. Fedro, and J. F. Mitchell, 1999, "Charge melting and polaron collapse in $\mathrm{La}_{1.2} \mathrm{Sr}_{1.8} \mathrm{Mn}_{2} \mathrm{O}_{7}$," Phys. Rev. Lett. 83, 4393-4396.

Vintaykin, Ye.Z., and V. Yu. Kolontsov, 1968, "Ageing of Fe-Cr alloys," Fiz. Met. Metalloved. 26, 282-288. 
Viret, M., H. Glättli, C. Fermon, A. M. de Leon-Guevara, and A. Revcolevschi, 1998, "Magnetic coherence above the Curie point in ferromagnetic LaSrMnO manganites," Europhys. Lett. 42, 301-306.

Viret, M., F. Ott, J. P. Renard, H. Glättli, L. Pinsard-Gaudart, and A. Revcolevschi, 2004, "Magnetic filaments in resistive manganites," Phys. Rev. Lett. 93, 217402.

Vivas, L. G., R. Yanes, and A. Michels, 2017, "Small-angle neutron scattering modeling of spin disorder in nanoparticles," Sci. Rep. 7, 13060.

Wagner, A., A. Ulbricht, F. Bergner, and E. Altstadt, 2012, "Influence of the copper impurity level on the irradiation response of reactor pressure vessel steels investigated by SANS," Nucl. Instrum. Methods Phys. Res., Sect. B 280, 98-102.

Wagner, W., and J. Kohlbrecher, 2005, in Modern Techniques for Characterizing Magnetic Materials, edited by Y. Zhu (Kluwer Academic Publishers, Dordrecht), pp. 65-105.

Wandersman, E., E. Dubois, F. Cousin, V. Dupuis, G. Mériguet, R. Perzynski, and A. Cbers, 2009, "Relaxation of the field-induced structural anisotropy in a rotating magnetic fluid," Europhys. Lett. 86, 10005.

Wang, B. M., Y. Liu, P. Ren, B. Xia, K. B. Ruan, J. B. Yi, J. Ding, X. G. Li, and L. Wang, 2011, "Large exchange bias after zero-field cooling from an unmagnetized state," Phys. Rev. Lett. 106, 077203.

Wang, X. L., C. T. Liu, U. Keiderling, A. D. Stoica, L. Yang, M. K. Miller, C. L. Fu, D. Ma, and K. An, 2012, "Unusual thermal stability of nano-structured ferritic alloys," J. Alloys Compd. 529, 96-101.

Wang, Zhentao, Yoshitomo Kamiya, Andriy H. Nevidomskyy, and Cristian D. Batista, 2015, "Three-Dimensional Crystallization of Vortex Strings in Frustrated Quantum Magnets," Phys. Rev. Lett. 115, 107201.

Weissmüller, J., R. D. McMichael, A. Michels, and R. D. Shull, 1999, "Small-Angle Neutron Scattering by the Magnetic Microstructure of Nanocrystalline Ferromagnets Near Saturation," J. Res. Natl. Inst. Stand. Technol. 104, 261.

Weissmüller, J., A. Michels, J. G. Barker, A. Wiedenmann, U. Erb, and R.D. Shull, 2001, "Analysis of the small-angle neutron scattering of nanocrystalline ferromagnets using a micromagnetics model," Phys. Rev. B 63, 214414.

Weissmüller, J., A. Michels, D. Michels, A. Wiedenmann, C. E. Krill, III, H. M. Sauer, and R. Birringer, 2004, "Spin structure of nanocrystalline terbium,” Phys. Rev. B 69, 054402.

Wetterskog, Erik, Cheuk Wai Tai, Jekabs Grins, Lennart Bergström, and German Salazar-Alvarez, 2013, "Anomalous magnetic properties of nanoparticles arising from defect structures: Topotaxial oxidation of $\mathrm{Fe}_{1-x} \mathrm{O}-\mathrm{Fe}_{3-\delta} \mathrm{O}_{4}$ core-shell nanocubes to singlephase particles," ACS Nano 7, 7132-7144.

White, J. S., S. P. Brown, E. M. Forgan, M. Laver, C. J. Bowell, R. J. Lycett, D. Charalambous, V. Hinkov, A. Erb, and J. Kohlbrecher, 2008, "Observations of the configuration of the high-field vortex lattice in $\mathrm{YBa}_{2} \mathrm{Cu}_{3} \mathrm{O}_{7}$ : Dependence upon temperature and angle of applied field," Phys. Rev. B 78, 174513.

White, J. S., et al., 2009, "Fermi Surface and Order Parameter Driven Vortex Lattice Structure Transitions in Twin-Free $\mathrm{YBa}_{2} \mathrm{Cu}_{3} \mathrm{O}_{7}$," Phys. Rev. Lett. 102, 097001.

White, J. S., et al., 2010, "Observations of Pauli paramagnetic effects on the flux line lattice in $\mathrm{CeCoIn}_{5}$, , New J. Phys. 12, 023026.

White, J. S., et al., 2011, "Magnetic-field-induced nonlocal effects on the vortex interactions in twin-free $\mathrm{YBa}_{2} \mathrm{Cu}_{3} \mathrm{O}_{7}$," Phys. Rev. B 84, 104519.

White, J. S., et al., 2012, "Electric field control of the skyrmion lattice in $\mathrm{Cu}_{2} \mathrm{OSeO}_{3}$," J. Phys. Condens. Matter 24, 432201.
White, J. S., et al., 2014, "Electric-Field-Induced Skyrmion Distortion and Giant Lattice Rotation in the Magnetoelectric Insulator $\mathrm{Cu}_{2} \mathrm{OSeO}_{3}$," Phys. Rev. Lett. 113, 107203.

Wiedenmann, A., 2001, "Small-angle neutron scattering investigations of magnetic nanostructures and interfaces using polarized neutrons," Physica B (Amsterdam) 297, 226-233.

Wiedenmann, A., 2002, in Ferrofluids: Magnetically Controllable Fluids and Their Applications, Lecture Notes in Physics, edited by S. Odenbach (Springer, New York), pp. 33-58.

Wiedenmann, A., 2010, "Static and dynamic correlations in magnetic nanomaterials studied by small angle neutron scattering techniques," Collection de la Société Française de la Neutronique 11, 219-242.

Wiedenmann, A., R. Gähler, C. D. Dewhurst, U. Keiderling, S. Prévost, and J. Kohlbrecher, 2011, "Relaxation mechanisms in magnetic colloids studied by stroboscopic spin-polarized smallangle neutron scattering," Phys. Rev. B 84, 214303.

Wiedenmann, A., A. Hoell, and M. Kammel, 2002, "Small-angle scattering investigations of cobalt-ferrofluids using polarised neutrons," J. Magn. Magn. Mater. 252, 83-85.

Wiedenmann, A., A. Hoell, M. Kammel, and P. Boesecke, 2003, "Field-induced pseudocrystalline ordering in concentrated ferrofluids," Phys. Rev. E 68, 031203.

Wiedenmann, A., M. Kammel, A. Heinemann, and U. Keiderling, 2006, "Nanostructures and ordering phenomena in ferrofluids investigated using polarized small angle neutron scattering," J. Phys. Condens. Matter 18, S2713-S2736.

Wiedenmann, A., U. Keiderling, K. Habicht, M. Russina, and R. Gähler, 2006, "Dynamics of Field-Induced Ordering in Magnetic Colloids Studied by New Time-Resolved Small-Angle NeutronScattering Techniques," Phys. Rev. Lett. 97, 057202.

Wiedenmann, A., U. Keiderling, M. Meissner, D. Wallacher, R. Gähler, R. P. May, S. Prévost, M. Klokkenburg, B. H. Erné, and J. Kohlbrecher, 2008, "Low-temperature dynamics of magnetic colloids studied by time-resolved small-angle neutron scattering," Phys. Rev. B 77, 184417.

Wiedenmann, Albrecht, 2005, "Polarized SANS for probing magnetic nanostructures," Physica B (Amsterdam) 356, 246-253.

Wiedenmann, Albrecht, and André Heinemann, 2005, "Fieldinduced ordering phenomena in ferrofluids observed by smallangle neutron scattering," J. Magn. Magn. Mater. 289, 58-61.

Wiedenmann, B., et al., 2017, "Reciprocal space mapping of magnetic order in thick epitaxial MnSi films," arXiv:1710.00544.

Wiesendanger, R., 2016, "Nanoscale magnetic skyrmions in metallic films and multilayers: a new twist for spintronics," Nat. Mater. 1, 16044.

Wiesendanger, Roland, 2009, "Spin mapping at the nanoscale and atomic scale," Rev. Mod. Phys. 81, 1495-1550.

Wignall, G. D., and Y. B. Melnichenko, 2005, "Recent applications of small-angle neutron scattering in strongly interacting soft condensed matter," Rep. Prog. Phys. 68, 1761-1810.

Wildes, A. R., 2006, "Scientific Reviews: Neutron Polarization Analysis Corrections Made Easy," Neutron News 17, 17.

Williams, W. G., 1988, Polarized Neutrons (Clarendon Press, Oxford).

Willis, B. T. M., and C.J. Carlile, 2009, Experimental neutron scattering (Oxford University Press, New York).

Wilson, M. N., E. A. Karhu, D. P. Lake, A. S. Quigley, S. Meynell, A. N. Bogdanov, H. Fritzsche, U. K. Rößler, and T. L. Monchesky, 2013, "Discrete helicoidal states in chiral magnetic thin films," Phys. Rev. B 88, 214420.

Woodcock, T. G., Y. Zhang, G. Hrkac, G. Ciuta, N. M. Dempsey, T. Schrefl, O. Gutfleisch, and D. Givord, 2012, "Understanding the 
microstructure and coercivity of high performance NdFeB-based magnets," Scr. Mater. 67, 536.

Woodward, F. M., J. W. Lynn, M. B. Stone, R. Mahendiran, P. Schiffer, J. F. Mitchell, D. N. Argyriou, and L. C. Chapon, 2004, "Field-induced avalanche to the ferromagnetic state in the phaseseparated ground state of manganites," Phys. Rev. B 70, 174433.

Wu, J., and C. Leighton, 2003, "Glassy ferromagnetism and magnetic phase separation in $\mathrm{La}_{1-x} \mathrm{Sr}_{x} \mathrm{CoO}_{3}$," Phys. Rev. B 67, 174408.

Wu, J., J. W. Lynn, C. J. Glinka, J. Burley, H. Zheng, J. F. Mitchell, and C. Leighton, 2005, "Intergranular giant magnetoresistance in a spontaneously phase separated perovskite oxide," Phys. Rev. Lett. 94, 037201.

Xia, Younan, Yujie Xiong, Byungkwon Lim, and Sara E. Skrabalak, 2009, "Shape-controlled synthesis of metal nanocrystals: Simple chemistry meets complex physics?" Angew. Chem., Int. Ed. Engl. 48, 60-103.

Xu, X., J. Odqvist, M. Hörnqvist, M. Thuvander, A. Steuwer, J. E. Westraadt, S. King, and P. Hedström, 2016, "Structural characterization of phase separation in Fe-Cr: A current comparison of experimental methods," Metall. Mater. Trans. A 47, 5942-5952.

Yaicle, C., et al., 2003, "Neutron scattering evidence for magneticfield-driven abrupt magnetic and structural transitions in a phaseseparated manganite," Phys. Rev. B 68, 224412.

Yamada, Y., T. Iwase, M. Watahiki, and J. Suzuki, 2001, "Small Angle Neutron Scattering Study on $\operatorname{Pr}_{0.7} \mathrm{Ca}_{0.3} \mathrm{MnO}_{3}$-Evidence of Two-Phase Coexistence-," J. Phys. Soc. Jpn. 70, 1593-1597.

Yano, M., K. Ono, M. Harada, A. Manabe, T. Shoji, A. Kato, and J. Kohlbrecher, 2014, "Investigation of coercivity mechanism in hot deformed Nd-Fe-B permanent magnets by small-angle neutron scattering," J. Appl. Phys. 115, 17A730.

Yano, M., K. Ono, A. Manabe, N. Miyamoto, T. Shoji, A. Kato, Y. Kaneko, M. Harada, H. Nozaki, and J. Kohlbrecher, 2012, "Magnetic Reversal Observation in Nano-Crystalline Nd-Fe-B Magnet by SANS," IEEE Trans. Magn. 48, 2804.

Yaouanc, A., P. Dalmas de Réotier, and E. H. Brandt, 1997, "Effect of the vortex core on the magnetic field in hard superconductors," Phys. Rev. B 55, 11107-11110.

Yaron, U., P. L. Gammel, D. A. Huse, R. N. Kleiman, C. S. Oglesby, E. Bucher, B. Batlogg, D. J. Bishop, K. Mortensen, and K. N. Clausen, 1995, "Structural evidence for a two-step process in the depinning of the superconducting flux-line lattice," Nature (London) 376, 753-755.

Yaron, U., P. L. Gammel, A. P. Ramirez, D. A. Huse, D. J. Bishop, A. I. Goldman, C. Stassis, P. C. Canfield, K. Mortensen, and M. R. Eskildsen, 1996, "Microscopic coexistence of magnetism and superconductivity in $\mathrm{ErNi}_{2} \mathrm{~B}_{2} \mathrm{C}$," Nature (London) 382, 236-238.

Yaron, U., et al., 1994, "Neutron Diffraction Studies of Flowing and Pinned Magnetic Flux Lattices in $2 \mathrm{H}-\mathrm{NbSe}_{2}$," Phys. Rev. Lett. 73, 2748-2751.

Yethiraj, M., D. K. Christen, D. McK. Paul, P. Miranovic, and J. R. Thompson, 1999, "Flux lattice symmetry in $\mathrm{V}_{3} \mathrm{Si}$ : Nonlocal effects in a high- $\kappa$ superconductor," Phys. Rev. Lett. 82, 5112-5115.

Yethiraj, M., D. McK. Paul, C. V. Tomy, and E. M. Forgan, 1997, "Neutron scattering study of the flux lattice in $\mathrm{YNi}_{2} \mathrm{~B}_{2} \mathrm{C}$," Phys. Rev. Lett. 78, 4849-4852.

Yethiraj, M., H. A. Mook, G. D. Wignall, R. Cubitt, E. M. Forgan, S. L. Lee, D. McK. Paul, and T. Armstrong, 1993a, "Anisotropic vortex lattice in $\mathrm{YBa}_{2} \mathrm{Cu}_{3} \mathrm{O}_{7}$," Phys. Rev. Lett. 71, 3019-3022.

Yethiraj, M., H. A. Mook, G. D. Wignall, R. Cubitt, E. M. Forgan, D. McK. Paul, and T. Armstrong, 1993b, "Small-angle neutron scattering study of flux line lattices in twinned $\mathrm{YBa}_{2} \mathrm{Cu}_{3} \mathrm{O}_{7}$," Phys. Rev. Lett. 70, 857-860.

Yoshimori, Akio, 1959a, "A New Type of Antiferromagnetic Structure in the Rutile Type Crystal," J. Phys. Soc. Jpn. 14, 807-821.

Yoshimori, Akio, 1959b, "La structure des substances magnetiques," J. Phys. Chem. Solids 11, 303-309.

Yosida, K., 1957, "Magnetic Properties of Cu-Mn Alloys," Phys. Rev. 106, 893-898.

Yu, X. Z., N. Kanazawa, Y. Onose, K. Kimoto, W. Z. Zhang, S. Ishiwata, Y. Matsui, and Y. Tokura, 2011, "Near room-temperature formation of a skyrmion crstal in thin-films of the helimagnet FeGe," Nat. Mater. 10, 106-109.

Yu, X. Z., Y. Onose, N. Kanazawa, J. H. Park, J. H. han, Y. Matsui, N. Nagaosa, and Y. Tokura, 2010, "Real-space observation of a twodimensional skyrmion crystal," Nature (London) 465, 901-904.

Yuan, S., P. L. Kuhns, A. P. Reyes, J. S. Brooks, M. J. R. Hoch, V. Srivastava, R. D. James, S. El-Khatib, and C. Leighton, 2015, "Magnetically nanostructured state in a Ni-Mn-Sn shape-memory alloy," Phys. Rev. B 91, 214421.

Yuan, S., P. L. Kuhns, A. P. Reyes, J. S. Brooks, M. J. R. Hoch, V. Srivastava, R. D. James, and C. Leighton, 2016, "Phase separation and superparamagnetism in the martensitic phase of $\mathrm{Ni}_{50-x} \mathrm{Co}_{x} \mathrm{Mn}_{40} \mathrm{Sn}_{10}$, , Phys. Rev. B 93, 094425.

Yusuf, S. M., J. M. De Teresa, M. D. Mukadam, J. Kohlbrecher, M. R. Ibarra, J. Arbiol, P. Sharma, and S. K. Kulshreshtha, 2006, "Experimental study of the structural and magnetic properties of $\gamma$ - $\mathrm{Fe}_{2} \mathrm{O}_{3}$ nanoparticles," Phys. Rev. B 74, 224428.

Zákutná, D., 2019, unpublished.

Zhang, X., Y. Zhou, and M. Ezawa, 2016, "Antiferromagnetic Skyrmion: Stability, Creation and Manipulation," Sci. Rep. 6, 24795.

Zhang, Z., R. D. James, and S. Müller, 2009, "Energy barriers and hysteresis in martensitic phase transformations," Acta Mater. 57, 4332-4352.

Zhang, Z. W., C. T. Liu, X.-L. Wang, K. C. Littrell, M. K. Miller, K. An, and B. A. Chin, 2011, "From embryos to precipitates: A study of nucleation and growth in a multicomponent ferritic steel," Phys. Rev. B 84, 174114.

Zheludev, A., S. Maslov, G. Shirane, Y. Sasago, N. Koide, K. Uchinokura, D. A. Tennant, and S. E. Nagler, 1997, "Square-lattice spiral magnet $\mathrm{Ba}_{2} \mathrm{CuGe}_{2} \mathrm{O}_{7}$ in an in-plane magnetic field," Phys. Rev. B 56, 14006-14012.

Zheludev, A., G. Shirane, Y. Sasago, N. Kiode, and K. Uchinokura, 1996, "Spiral phase and spin waves in the quasi-two-dimensional antiferromagnet $\mathrm{Ba}_{2} \mathrm{CuGe}_{2} \mathrm{O}_{7}$," Phys. Rev. B 54, 15163-15170.

Zheludev, A., G. Shirane, Y. Sasago, N. Koide, and K. Uchinokura, 1997, "Spiral order in $\mathrm{Ba}_{2} \mathrm{CuGe}_{2} \mathrm{O}_{7}$," Physica B (Amsterdam) 234 236, 546-548.

Zhong, S. Y., V. Klosek, Y. de Carlan, and M. H. Mathon, 2016, "Modeling of structural hardening in oxide dispersion strengthened (ODS) ferritic alloys," J. Mater. Sci. 51, 2540-2549.

Zighem, F., F. Ott, T. Maurer, G. Chaboussant, J.-Y. Piquemal, and G. Viau, 2013, "Numerical calculation of magnetic form factors of complex shape nanoparticles coupled with micromagnetic simulations," Phys. Procedia 42, 66-73.

Zinkle, S. J., and G. S. Was, 2013, "Materials challenges in nuclear energy," Acta Mater. 61, 735-758.

Živković, I., J. S. White, H. M. Rønnow, K. Prša, and H. Berger, 2014, "Critical scaling in the cubic helimagnet $\mathrm{Cu}_{2} \mathrm{OSeO}_{3}$," Phys. Rev. B 89, 060401. 